\title{
Application of pyrrolidone building blocks in developing renewable 2-oxazolines based thermosets
}

Citation for published version (APA):

Roy, M. (2020). Application of pyrrolidone building blocks in developing renewable 2-oxazolines based thermosets: Towards sustainable and recyclable resins. [Doctoral Thesis, Maastricht University]. Maastricht University. https://doi.org/10.26481/dis.20200824mr

Document status and date:

Published: 01/01/2020

DOI:

$10.26481 /$ dis.20200824mr

Document Version:

Publisher's PDF, also known as Version of record

\section{Please check the document version of this publication:}

- A submitted manuscript is the version of the article upon submission and before peer-review. There can be important differences between the submitted version and the official published version of record.

People interested in the research are advised to contact the author for the final version of the publication, or visit the DOI to the publisher's website.

- The final author version and the galley proof are versions of the publication after peer review.

- The final published version features the final layout of the paper including the volume, issue and page numbers.

Link to publication

\footnotetext{
General rights rights.

- You may freely distribute the URL identifying the publication in the public portal. please follow below link for the End User Agreement:

www.umlib.nl/taverne-license

Take down policy

If you believe that this document breaches copyright please contact us at:

repository@maastrichtuniversity.nl

providing details and we will investigate your claim.
}

Copyright and moral rights for the publications made accessible in the public portal are retained by the authors and/or other copyright owners and it is a condition of accessing publications that users recognise and abide by the legal requirements associated with these

- Users may download and print one copy of any publication from the public portal for the purpose of private study or research.

- You may not further distribute the material or use it for any profit-making activity or commercial gain

If the publication is distributed under the terms of Article $25 \mathrm{fa}$ of the Dutch Copyright Act, indicated by the "Taverne" license above, 


\section{Application of pyrrolidone building blocks in developing renewable 2-oxazolines based thermosets}

Towards sustainable and recyclable resins

\section{DISSERTATION}

To obtain the Doctoral degree at Maastricht University, on the authority of the Rector Magnificus,

Prof. dr. Rianne M. Letschert

in accordance with the decision of the Board of Deans,

to be defended in public

on Monday $24^{\text {th }}$ August, 2020, at 10:00 hours.

by

Manta Roy 
Supervisor:

Prof. dr. Sanjay Rastogi

Co-supervisor:

Dr. Karel Wilsens

Assessment Committee:

Prof. dr. Maarten Honing ( chair)

Prof. dr. Filip Du Prez, Ghent University, Belgium

Prof. dr. Katja Loos, University of Groningen

Dr. Matt Baker 
Application of pyrrolidone building blocks in developing renewable 2-oxazolines based thermosets

Towards sustainable and recyclable resins

Doctoral thesis

Manta Roy

Aachen-Maastricht Institute for Biobased Materials, Geleen.

Maastricht University 
Application of pyrrolidone building blocks in developing renewable 2-oxazolines based thermosets: Towards sustainable and recyclable resins

Manta Roy, Maastricht University, 2020

ISBN: 978-94-6380-891-0

(C) 2020, Manta Roy

Printed by Proefschriftmaken

Cover art and layout designed by Anand H.S Iyer and Manta Roy

This thesis work is part of the research program and funded by Aachen-Maastricht Institute of Biobased materials (AMIBM) and has received funding from the INTERREG V program Flanders-Netherlands (Puur Natuur: 100\% Biobased), the cross-border collaboration program financially supported by the European fund for regional development. 


\section{GLOSSARY}

+ I effect Inductive effect

$+\boldsymbol{R}$ effect Resonance stabilizing effect

11-und PD 11-aminoundecanoic acid based pyrrolidone dicarboxylic acid

2,5-FDCA 2,5-furandicarboxylic acid

$3 \boldsymbol{R} \quad$ Reduce, reuse, recycle

$\mathbf{6} \quad$ Reduce, reuse, recycle, redesign and remanufacture

ATR-FTIR spectroscopy

BHEF N,N'-bis(2-hydroxyethyl)furan-2,5-dicarboxamide

BHET N,N'-bis(2-hydroxyethyl)terephthalamide

BHFDA N,N-bis(2-hydroxyethyl)furan-2,5-dicarboxamide

BP-C8 N,N'-octamethylene-bis(pyrrolidone-4-carboxylic acid)

BP-Cx Bis(pyrrolidone) dicarboxylic acids with ' $x$ ' carbon spacer length

CDCl3 deuterated chloroform

CE Circular economy

D2O deuterated water

DMSO-d6 deuterated dimethyl-sulfoxide

E factor Environmental factor

EWG Electron withdrawing group

FDCAox 2,5-FDCA based bis(2-oxazoline)

FDCAox 2,5-bis(4,5-dihydrooxazol-2-yl)furan

FTIR Fourier-transform infrared spectroscopy

GPC Gel permeation chromatography

HFIP 1,1,1,3,3,3-hexafluoroisopropanol

IA Itaconic acid

IAda N,N-bis(2-hydroxyethyl)benzene-1,3-dicarboxamide

IAox 1,3-bis(4,5-dihydrooxazol-2-yl)benzene

L-ala-PD L-alanine based pyrrolidone dicarboxylic acid

$L-D O P A-$

PD L-Dopamine based pyrrolidone dicarboxylic acid

L-his-PD L-histidine based pyrrolidone dicarboxylic acid

L-lys-PD L-lysine based pyrrolidone dicarboxylic acid

L-ph-ala-

$P D$

L-phenyl alanine based pyrrolidone dicarboxylic acid

L-tyr-PD L-tyrosine based pyrrolidone dicarboxylic acid 


\begin{tabular}{|c|c|}
\hline$M C T$ & Mercury cadmium telluride detector \\
\hline$M w$ & Molecular weight \\
\hline NMR & Nuclear magnetic resonance \\
\hline$P F G$ & Pendant functional groups \\
\hline$P L A$ & Polylactic acid \\
\hline $\boldsymbol{R H}$ & Relative humidity \\
\hline $\operatorname{SeA}$ & Sebacic acid \\
\hline$T F A-d$ & deuterated trifluoroacetic acid \\
\hline$T g$ & Glasstransition temperature \\
\hline$T G A$ & Thermogravimetric analysis \\
\hline$T m$ & Melting temperature \\
\hline$T M S$ & tetramethylsilane \\
\hline TNBS & 1,4,6-trinitrobenzenesulphonic acid \\
\hline TPP & triphenylphosphite \\
\hline Tris buffer & tris(hydroxymethyl)aminomethane \\
\hline$U P E$ & Unsaturated polyester \\
\hline$U \boldsymbol{V}$-vis & Ultraviolet visible range \\
\hline$\beta$-ala-PD & $\beta$-alanine based pyrrolidone dicarboxylic acid \\
\hline$\gamma-a b a-P D$ & $\gamma$-aminobutyric acid based pyrrolidone dicarboxylic acid \\
\hline
\end{tabular}


Dedicated to my parents

and my husband 



\section{Table of Contents}

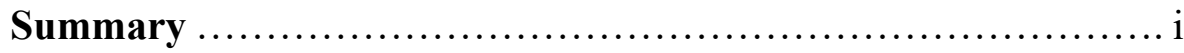

Chapter 1: Introduction ..................................... 1

1.1 Linear to circular economy ........................................... 3

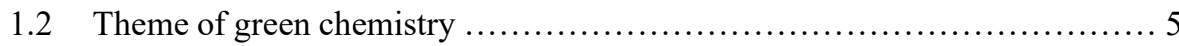

1.3 Biomass as an alternative renewable source ............................ 6

1.4 Michael addition reaction ............................................ 7

1.5 Oxazolines - A class of heterocyclic ring .............................. 10

1.6 Renewable pyrrolidone monomers to polymers ......................... 11

1.7 Reversible dynamic networks ......................................... 14

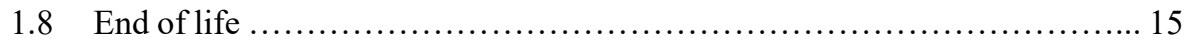

1.9 Aim and scope of the thesis ........................................... 16

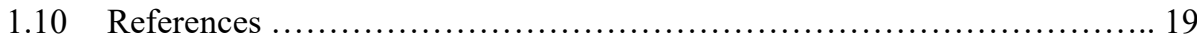

Chapter 2: Renewable (bis)pyrrolidone monomers as components for thermally curable and enzymatically depolymerizable 2-

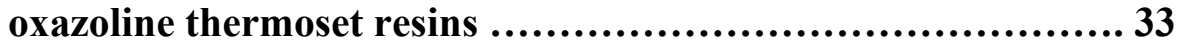

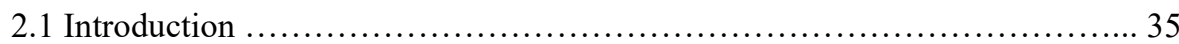

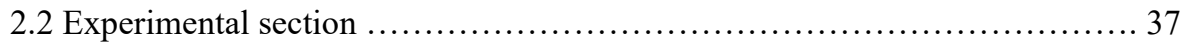

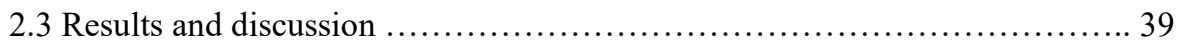

2.3.1 Bis(pyrrolidone) monomer synthesis and characterizations .... 39

2.3.2 In-situ monitoring of curing performance with bis(oxazolines) 43

2.3.3 Thermal behavior of developed thermosets .................... 51

2.3.4 Enzymatic depolymerization: Towards predicting end of life ... 52

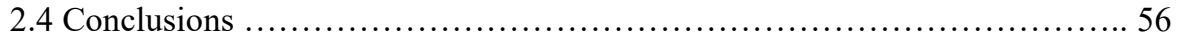

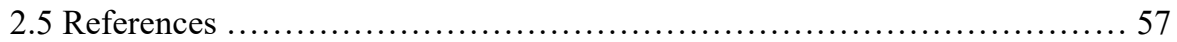




\section{Chapter 3: Renewable (Pyrrolidone)-Based Thermosets: \\ Synthesis, Characterization, and Potential Route for Their}

Chemical Recycling ............................................ 63

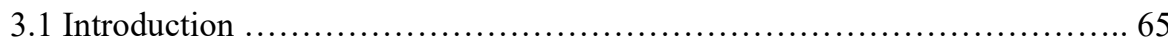

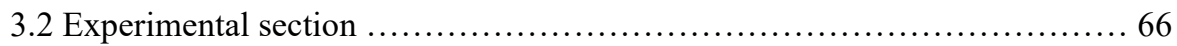

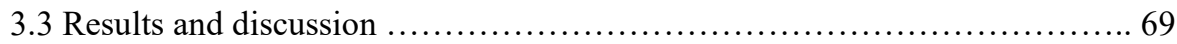

3.3.1 Synthesis, characterization and thermal behavior of developed thermosets

3.3.2 Effect of moisture of its hydrolytic stability and mechanical performance .................................................... 74

3.3.3 Application and evaluating its performance as adhesives ...... 77

3.3.4 Employing hydrolysis towards controlling thermoset properties and chemical recycling of synthesized monomer ....................... 80

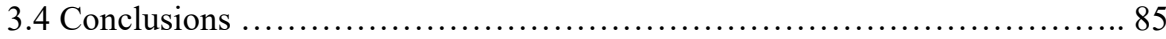

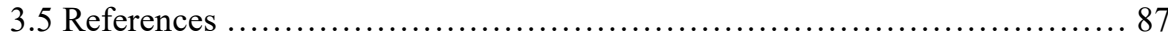

Chapter 4: The Aza-Michael addition-cyclization reaction;

Synthesis of (functional) pyrrolidone dicarboxylic acids from

naturally occurring amino acids, their polymerization, and

viscoelastic behavior .......................................... 93

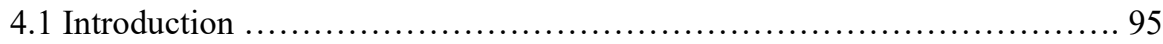

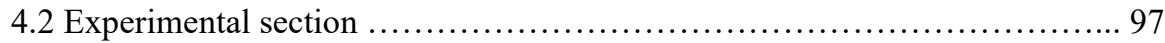

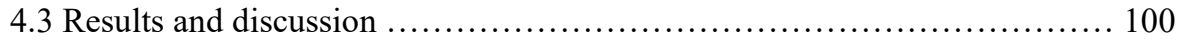

4.3.1 Aza-Michael cascade reaction on naturally occurring amino acids: Synthesis and characterization of functional monomers ........... 100

4.3.2 Thermal ring opening polymerization with bis(oxazolines) ... 117

4.3.3 Thermal stability of synthesized functional monomers ......... 120

4.3.4 Rheology and stress relaxation ............................. 121 


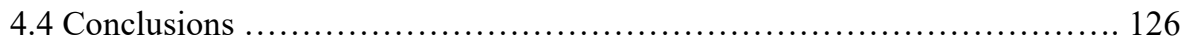

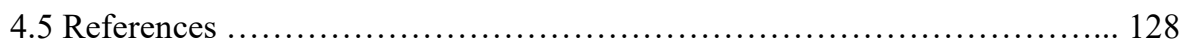

Chapter 5: Evaluating the performance of amide based organic compounds as plasticizer and nucleating agent in polylactide 133

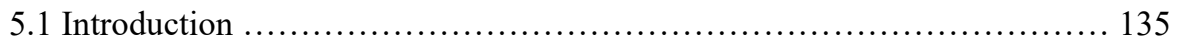

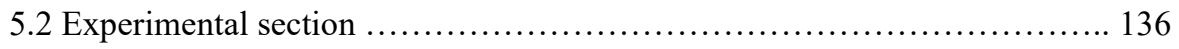

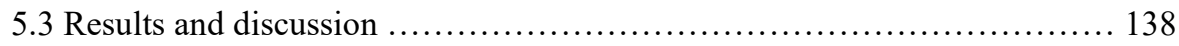

5.3.1 Influence of processing conditions on molecular weight of PLA ...138

5.3.2 BHET and BHEF as nucleating agents for Polylactic acid......... 141

5.3.3 Effect of cooling rate on BHEF morphology and nucleating efficiency of PLA.............................................................. 146

5.4 Conclusions ......................................................... 150

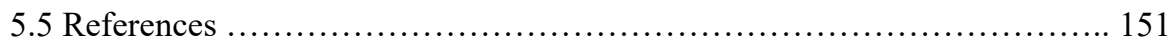

Chapter 6: On the nucleation of polylactide by melt-soluble

oxalamide based organic compounds ....................... 155

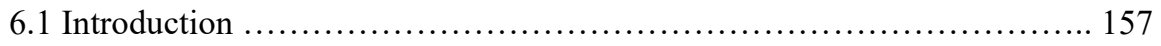

6.2 Experimental section ............................................... 158

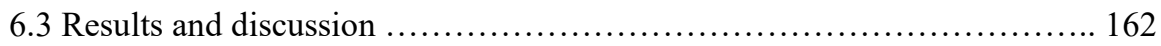

6.3.1 Bisoxalamides as nucleating agents for PLA ................ 162

6.3.2 Effect of its crystallization temperature on transcrystalline PLA morphology

6.3.3 PLA crystallization during cooling in presence of OBOC I varying concentration .................................................. 168

6.3.4 Identifying OBOC nucleation mechanism .................... 170

6.3.5 Approximation of shear rates during OBOC crystal growth .. 172

6.3.6 Optimizing processing conditions for improved PLA nucleation 


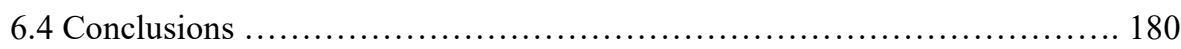

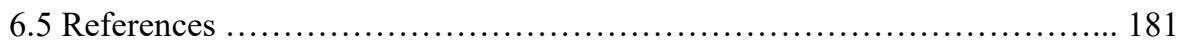

Chapter 7: Epilogue ........................................... 187

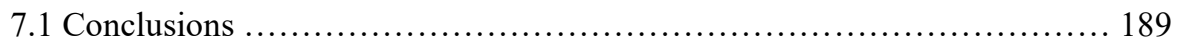

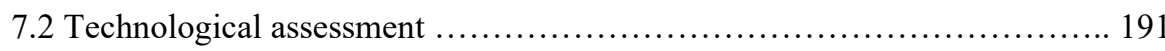

7.2.1 Adhesives in coatings and bio-medical applications ........... 191

7.2.2 Transition from traditional to sustainable (biobased) resin .. 192

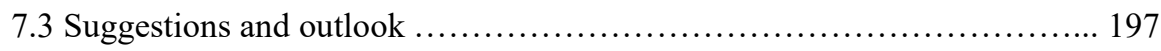

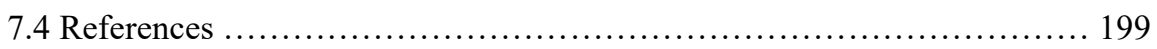

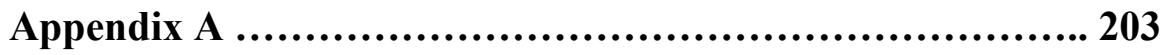

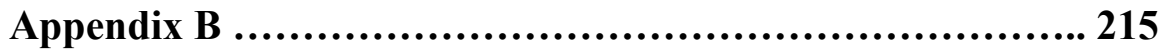

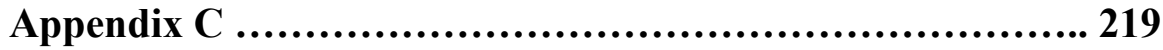

Appendix D ............................................... 229

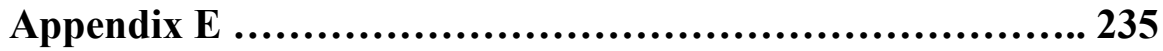

List of publications ......................................... 241

Acknowledgements .......................................... 243

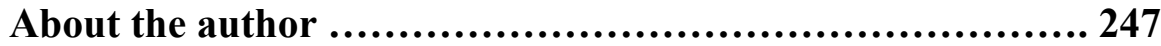




\section{Summary}

Polymers, both thermoplastics and thermosets, are of high importance in our daily life. However, the concern arising due to depletion of fossil feedstocks and traditional usage of plastics have forced both academia and industry to explore sustainable routes to develop polymers following biobased circular economy principles, i.e. through development of degradable, reusable, or recyclable polymers. To this end, biobased molecules synthesized from biobased/bio-sources or renewable feedstocks provide an alternative monomer source for polymers. One such interesting monomer is itaconic acid, with two functional dicarboxylic acids and one unsaturated bond, allowing it to undergo aza-Michael addition-cyclization (cascade) reaction, thereby generating a five membered heterocyclic pyrrolidone dicarboxylic acids functionality. Some of these pyrrolidone monomers have been recently used to develop fully renewable, degradable polymers like polyesters and poly amides, however the functional and reactive pyrrolidone monomer synthesis and its purifications are not yet optimized and explored. Traditional thermoset resins are non-recyclable, non-degradable which thereby end up as landfills or garbage thrown into oceans due to their permanent covalent crosslinking. Until now, epoxy resins have been extensively used to develop thermosets. However, there are several drawbacks of using epoxy resins, for example - its toxicity, brittleness, nonrecyclability and poor mechanical performance. Retrospectively, bis(oxazolines) chemistry has proven its beneficial potential in developing linear and branched polymers over decades. Until now, these bis(oxazolines) are widely used as chain extenders, however, their potential in developing thermosetting resins is hardly explored. Furthermore, their curing kinetics, mechanical performances and their potential end-of-life has not been evaluated. Additionally, the green pathway for synthesis of these bis(oxazolines) makes them more interesting, while following the concepts of green chemistry and sustainability principles.

In this thesis, the potential of aza-Michael addition-cyclization (cascade) reaction is investigated with the aim to optimize the synthetic conditions and purification of various symmetric and multi-functional pyrrolidone dicarboxylic acids. They are further reacted with furan and phenyl based bis(oxazolines) to undergo ring opening polymerization, with the focus to understand their curing kinetics, mechanical properties under ambient and various controlled humidity conditions, and evaluating their potential end of life. Hence bis(oxazolines) chemistry is investigated thoroughly to develop both thermoplastics and thermosets, when reacted with the synthesized pyrrolidone dicarboxylic acids. The study also focuses on exploring the potential of renewable pyrrolidone dicarboxylic acids as new biobased building blocks for generation of bio-based low viscous thermosetting resins, used as an adhesive. While performing this study the efforts are made to understand 
1) the synthesis and purification steps following the requirements of green chemistry;

2) the curing kinetics of both linear chain formation and crosslinking density; 3) influence of ambient condition and relative humidity on the mechanical performances of the developed thermoplastic and thermoset; 4) potential end of life - recyclability, enzymatic depolymerization followed with recovery and reusage of the renewable monomers. This knowledge of understanding is relevant in developing a toolbox of novel renewable and recyclable thermoset resins/thermoplastics by using renewable bio-based building blocks.

The symmetrical (bis)pyrrolidone dicarboxylic acids synthesis and its purification obtained by using itaconic acid and various aliphatic diamines via azaMichael addition reaction is performed and investigated using a combination of ${ }^{1} \mathrm{H}$ and 2D-NMR (structural analysis) and thermal analysis (DSC and TGA) (Chapter 2). Through NMR, GPC, and FTIR analysis it is demonstrated that these (bis)pyrrolidone dicarboxylic acids exhibit significantly enhanced curing rates in 2oxazoline resins compared to resins containing aliphatic dicarboxylic acids such as sebacic acid. Overall, it is shown that the rate of 2-oxazoline ring opening addition with carboxylic acid functionalities is determined by the dicarboxylic acid, whereas the ring opening addition of the 2-oxazoline functionality with amide groups is determined by the bis(2-oxazoline) compound. The thermosets obtained after curing proved to be readily plasticized by water, thereby opening possibilities towards predicting its potential end of life via enzymatic degradation.

Followed with the understanding of their curing kinetics, several thermosets with varying crosslinking densities are prepared by mixing the desired ratio of (bis)pyrrolidone dicarboxylic acid with bis(oxazolines), followed by thermal curing at $180^{\circ} \mathrm{C}$ (Chapter 3). The crosslink density of the thermosets is controlled by the usage of an excess of bis(2-oxazoline) and the achievement of full conversion is confirmed by Fourier transform infrared spectroscopy (FTIR). Retrospectively, the glass transition temperature of the thermosets increases with increasing crosslinking density. Nevertheless, all synthesized thermosets are prone to absorb water upon storage, resulting in a strong plasticizing effect and thus a suppression of the glass transition temperature. To assess the role of water on the thermo-mechanical properties, all thermosets are exposed to various relative humidity and their mechanical performance is evaluated. In general, we observe that the suppression in $T_{g}$ under the influence of water facilitates a brittle-to-ductile transition as the $T_{g}$ of the thermosets approaches room temperature, without significantly facilitating hydrolysis of the polymer backbone. Furthermore, it was observed that hydrolysis of the ester bonds can be enforced at elevated temperatures and high pressure in the presence of water, resulting in liberation of the used renewable (bis)pyrrolidone monomer in aqueous phase. Interestingly, the renewable (bis)pyrrolidone 
dicarboxylic acids can readily be isolated by filtration as it crystallizes from water during cooling, thereby providing a promising route for its chemical recycling. Additionally, these renewable resins have better adhesive property to glass, metals and any polar surfaces having amide or ester bonds. Thus, the adhesion performance of these renewable thermoset resins is evaluated on aluminium and polylactide (PLA), both under ambient condition and after immersion in water that resulted in promising findings. Glass is excluded from the plan of experiment due to safety concerns.

Furthermore, the effect of additional functionality and reactive groups towards ring opening polymerization with bis(oxazolines) is studied while using the synthesized functional pyrrolidone dicarboxylic acids obtained by using itaconic acids and naturally occurring L-amino acids (Chapter 4). The developed monomers prove to be excellent candidates for thermal polymerization with bis(2-oxazolines), yielding thermally stable thermoplastic polymers and thermosets. Through GPC and NMR analysis, it is demonstrated that the aromatic alcohol group in the monomer derived from L-tyrosine is non-reactive towards thermal ring opening addition with 2-oxazolines under the employed reaction conditions. In other words, the thermal ring-opening addition polymerization of bis(2-oxazolines) can effectively be used to generate (co)polymers with pendant aromatic alcohol groups without requiring any protecting groups or solvents. Through evaluation of the visco-elastic response and stress relaxation tests, it can be proved that the relaxation behaviour of the developed polymers can be tailored by introduction of pendant functional groups, effectively increasing the secondary interactions.

As explained earlier in Chapters $\mathbf{2}$ and $\mathbf{3}$ on the performance of 2-oxazoline based polymers, together with their potential for hydrolysis and consecutive recovery of the (bis)pyrrolidone dicarboxylic acids, the role of the residual diol of the ringopened 2-oxazoline has been ignored. However, the presence of two hydroxyl groups combined with two amide moieties makes these organic compounds promising candidates as nucleating agents; because they exhibit high melting temperature due to strong hydrogen bonding and a strong ability to self-assemble/crystallize upon cooling. Thus, the potential of bis(2-hydroxyethyl)furan-2,5-dicarboxamide (BHEF) and N,N'-bis(2-hydroxyethyl)terephthalamide (BHET) are explored as additives for PLA (Chapter 5). Overall it demonstrates that the BHET compound, potentially isolated from chemical recycling of thermally cured polymers, based on terephthalic acid based bis(2-oxazoline), is a promising additive for PLA (acting both as a plasticizer and nucleating agent, depending on the amount used), whereas, BHEF, the product that can be isolated after hydrolysis of thermally cured polymers containing a 2,5-furandicarboxylic acid based bis(2-oxazoline) has, despite its renewable origin, no signifnant effect as additive for PLA. Hence this renewable monomer (BHEF) is 
better suited for the development of bio-based and biodegradabe polymers rather than for reuse as additive for PLA after chemical recyling.

While working with BHET and BHEF as nucleating agents for PLA, it is observed that the cooling rate and the amount ( $\mathrm{wt} \%$ ) of NA used plays a significant role in enhancing the nucleation efficiency of the matrix (PLA). Thus to further investigate the effect of supercooling, a series of oxalamide based organic compounds (OBOC) are synthesized and their capability to enhance the nucleation of polylactide is evaluated (Chapter 6). Interestingly, it is observed that the nucleation efficiency of the OBOCs increases at high cooling rates, making them more attractive for industrial processing conditions. It is observed that the nucleation efficiency of polylactide is significantly enhanced in the presence of OBOC crystals, as a transcrystalline PLA morphology grows from the OBOC crystal surface (at relatively low supercooling from the equilibrium melting temperature of PLA). However, such crystallization of PLA occurs only when the OBOC crystals are formed at or below $145^{\circ} \mathrm{C}$ while cooling at $10{ }^{\circ} \mathrm{C} / \mathrm{min}$. In contrast, when the OBOC crystals are formed above $145^{\circ} \mathrm{C}$ (i.e. from a lower supersaturated state), the nucleation efficiency is reduced. Thus these findings override the possibility of both epitaxy and soft-epitaxy as their only plausible nucleation mechanism. Supported with in-situ polarized optical microscopy, differential scanning calorimetry, plate-plate rheology and molecular modelling, it is confirmed that the enhanced nucleation results from local stresses imposed with crystal growth of the nucleating agent on the polylactide melt thereby invoking stress-enhanced nucleation. Thus the reasoning of nucleation efficiency is attributed to 1) The imposed local shear rates, facilitated by the rapid growth of the OBOC crystals, which are high enough to facilitate contour orientation of the high molecular weight PLA chains next to the growing OBOC crystals, confirming the possibility for stress-enhanced nucleation, and 2) surface roughness of OBOC crystals. OBOC crystallization at high supersaturation yields defected smaller/defected OBOC crystals, which provides higher surface area and surface roughness, in contrast to lowered supersaturation $\left(>150^{\circ} \mathrm{C}\right)$, they undergo annealing thereby providing a smoother surface and lesser surface area - retrospectively exhibiting a decreased nucleation efficiency, irrespective of the PLA supercooling. Interestingly, both the surface roughness of OBOC crystals and the local stresses imposed on the PLA melt increase when the OBOC crystal growth proceeds from a highly supersaturated state, providing an explanation to the cause of the favored crystallization of PLA at high cooling rates in presence of the chosen oxalamides.

In this thesis, the potential of pyrrolidone based dicarboxylic acids is explored elaborately in developing renewable thermplastics and thermosetting resins. The thesis conclusively demonstrates that the combination of renewable pyrroldione dicarboxylic acids (both symmetrical and functional) together with potential biobased 
bis(oxazolines) is a promising alternative in developing fast curable, biobased resins with controlled curing kinetics. The control over curing kinetics is highly desired in order to achieve optimal mechanical performance. Furthermore, the curing chemistry of bis(oxazolines) and pyrroldione dicarboxylic acids proved to be also beneficial in developing functional and renewable biobased thermosetting resins with additional functionality as pendant groups, available for secondary interactions thereby enabling dynamic networks formation. Furthermore, these renewable resins have better adhesion performance with metal and PLA, both under ambient conditions and after immersion in water. Due to the presence of numerous polar groups, the developed renewable thermosets and thermoplastics are prone to moisture absorption and thus can undergo hydrolysis under extreme condition, enabling to predict its end of life via enzymatic depolymerization and recycling of the developed polymer. Furthermore, recovery and reusage of these renewable pyrrolidone monomer is a promising addition towards circularity, on the other hand the potential of the open diols, obtained from ring opened structure of used bis(oxazolines), is further used as an additive (nucleating agent) for PLA to enhance its nucleation efficiency. Thus this thesis demonstrates that the concept of biobased circularity can be achieved while using these renewable pyrrolidone monomers in developing 2-oxazoline based polymers. These scientific reaserch findings, investigated with specific organic compunds or systems, are of general nature, thus can be also applied to develop other polymer systems. 



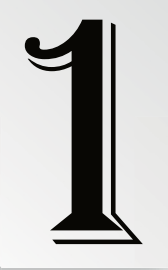

INTRODUCTION 


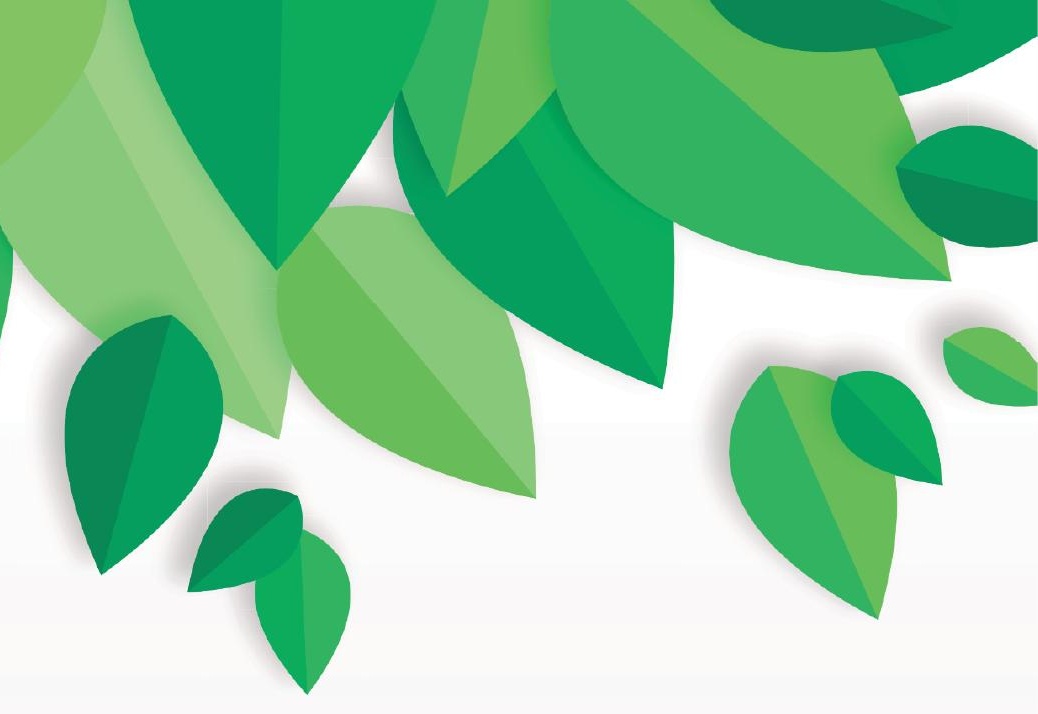

"What we are doing matters more

than what we used to do"

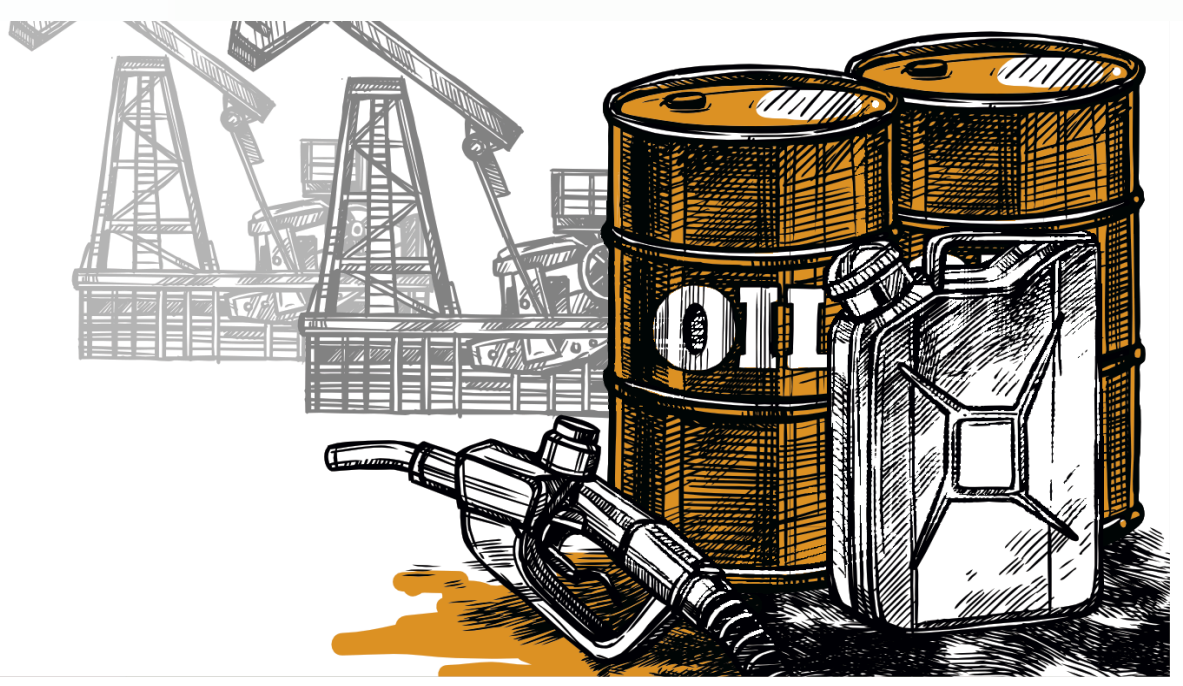




\subsection{Linear to circular economy}

\subsubsection{Transition from a linear to circular economy}

For several decades, a linear economy has been in practice, which means that raw materials are transformed into products, are thrown away after use, and generally end up with aquatic life or as landfill. ${ }^{1}$ To limit the generation of waste and employ residual value of the products, recycling of materials or products came into practice $^{2}$. Owing towards the environmental consequences of linear production and consumption of single used plastics in our daily life, the concept and practice of implanting a circular economy has gained momentum (figure 1.1) ) $^{3,4}$. In a circular economy, manufacturers design products that are reusable, easier to repair and/or recover the used resources. While practicing this concept of closing the loop (i.e. making material streams circular rather than linear), the life span of these products extends and consumption of natural resources can be controlled or monitored ${ }^{5}$. Current examples include material recycling for reusage of used products, lowering the usage of natural resources in existing processed, and rethinking our value chains towards more renewable resource efficiency ${ }^{6}$. Our planet has a finite supply of fossilbased resources and limited annual supply of renewable resources; serious problems are anticipated as the continuous consumption of "seeming to be - abundant" natural resources proceeds to fulfill humankind's ever-increasing demands and needs ${ }^{7}$. These activities include extraction, processing, manufacturing and usage activities, with a larger part of the used products ending up in landfill ${ }^{8}$. Hence, the concept and practice of a circular economy (CE) provides every tool, as it is based on three rules -1) reducing wasteful resources, 2) effective design and implementation of products and 3) improved resource efficiency, which is combined together with circular material flow involving recovery, reuse, recycling and remanufacturing (3R concept) of processed products ${ }^{5,3}$.

Thus, this practice is not only our sole option, but also it is considered as an inevitable step towards conservation of our natural resources, maintaining an ecological biodiversity, balanced human life and economic growth. The concept of circular economy developed over time. It started a few decades ago and started with the concepts of 1R (Reduce) introduced in the $1980 \mathrm{~s}^{5}$. Later, during the 1990s, the 3R concept, Reduce, Reuse and Recycle became a practice for focus. ${ }^{8,9,10}$ Nowadays, these concepts are developed further and aim to optimize the use of utilized natural resources, reducing pollution, emissions and waste. The current trend shows that achieving sustainable value chain in manufacturing requires green to sustainable manufacturing process ${ }^{11,12}$. 


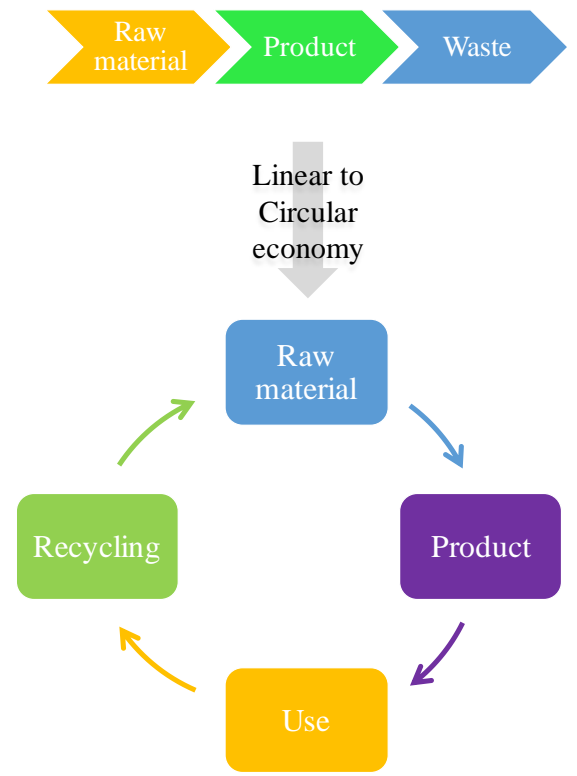

Figure 1.1. Schematic overview of the practices in a linear, reuse, and circular economy.

\subsubsection{Approach towards Closed-loop Sustainability}

Focusing towards sustainable practices while manufacturing products, leads to broader methodology for product development over multiple life cycles ${ }^{5,7,2}$. In this regards, as stated by I.S Jawahir the 3 Rs principle are extended further to the $6 \mathrm{R}$ principle, Reduce, Reuse, Recycle, Recover, Redesign, and Remanufacture (figure 1.2), which offers a closed loop with multiple extended life cycle systems as a basis for sustainable process 5 . While explaining 6R principles, the Reduce terminology focuses more on reduced energy, materials and other natural resources during its manufacturing process, while taking into account the reduction in emissions and wastes during usage or production ${ }^{5}$. Reuse refers to reuse of products as a whole or components after its first life cycle, and for its consequent cycles, reducing the virgin materials usage while producing newer products. Recycle involves the practices of considering the generated wastes used in developing new materials or products. Recovery is the process of collection, assembling, grading and cleaning of the used products at their end of use, while, Redesigning involves the rethinking to develop next generation products obtained from the previous products. This step is more beneficial from sustainable point of view, in order to avoid landfill. Lastly, Remanufacturing involves the reprocessing of the used products aiming towards regaining their original state without loss of their performance or functionality. 
Hence, the $6 \mathrm{R}$ principles can provide a powerful basis for a more sustainable use of materials.

\subsection{Theme of Green Chemistry}

To address the sustainability in the field of chemistry, the 12 principles of Green chemistry have been introduced by Anastas and Warner ${ }^{13}$ about 30 years ago. It has taken a paradigm shift from traditional concepts of process efficiency (only focused on chemical yield), to that of one assigning the economic value by eliminating the waste and avoiding the usage of toxic and/or hazardous substances ${ }^{7}$. In this regard, green chemistry has become an utmost demanding research topic since its introduction. A useful parameter to measure the environmental acceptability of chemical processes is $\mathrm{E}$ (Environmental) factor, which defines the ratio of the waste to desired product formation ${ }^{14,15,16 \text {, }}$

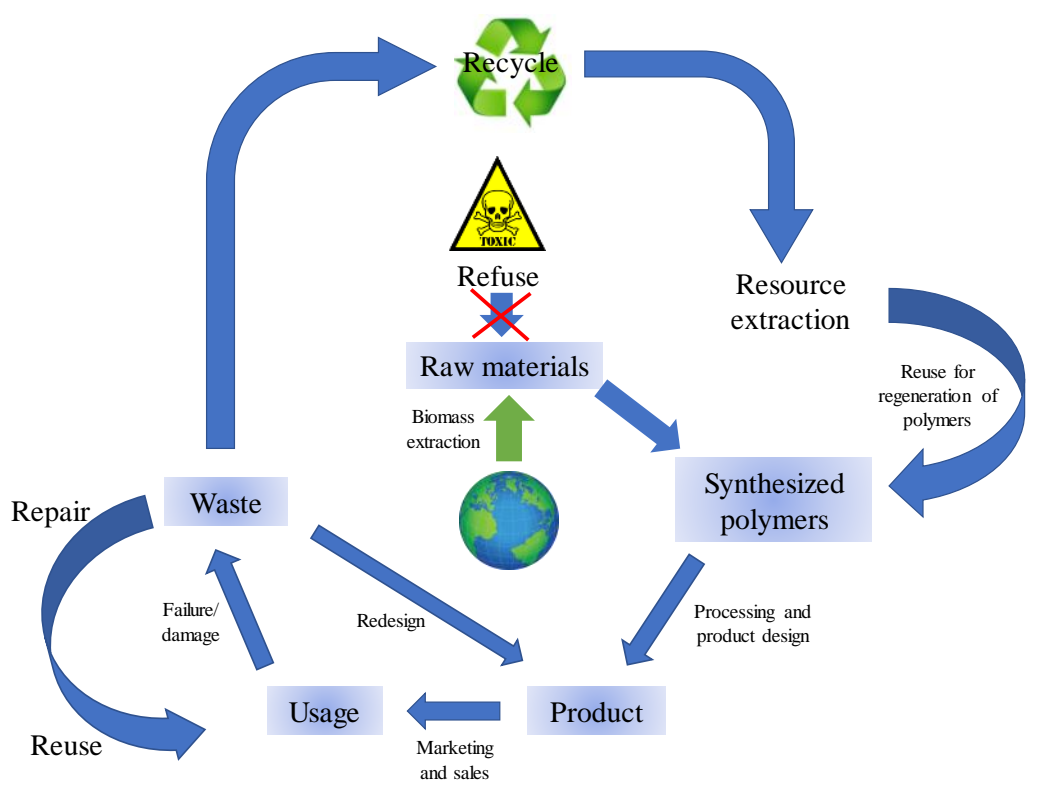

Figure 1.2. Closing the loop while practicing the 6R principles for sustainable and green polymeric future.

During the last two decades, the concept of green chemistry has been widely embraced by both academia and industry ${ }^{17}$. As a working definition of the essence of green chemistry, once Roger A. Sheldon ${ }^{16}$ simplified it to a single sentence: 
"Green chemistry efficiently utilizes (preferably renewable) raw materials, eliminates waste and avoids the use of toxic and/or hazardous reagents and solvents in the manufacture and application of chemical products"

Hence, one could say that sustainability is our prime goal and green chemistry is a route to achieve it. Accordingly, the focus in this thesis is on development and evaluation of new monomers and polymers from renewable resources while taking the concepts of green chemistry into consideration. Furthermore, identification of the materials properties and providing routes for their reuse or recycling is essential to predict their sustainability and is therefore a second aim of this work.

\subsection{Biomass as an alternative renewable source}

\subsubsection{Itaconic acid -A renewable biobased building block}

Biomass is considered as the only source for renewable organic carbon on earth; it offers an efficient platform for the production of bioorganic acid as an alternative to the fast depletion of non-renewable fossil based resources. ${ }^{18,19,20,21}$ In this regard, itaconic acid (IA) is chosen as an efficient biobased alternative synthesized from, for example, lignocellulose biomass ${ }^{22,23,24}$. IA production can take place through various ways like fermentation of sugars by fungi like Aspergillus terreus $^{25,26,27}$ and Ustilago maydis ${ }^{22}$ strains or, by metabolically engineered bacteria like Escherichia coli $^{22}$ and Corynebacterium glutamicum ${ }^{22}$. Itaconic acid was discovered first by S. Baup in 1836 as it is a thermal decomposition product of citric $\operatorname{acid}^{28}$, while its production by Aspergillus itaconicus was reported by Kinoshita in $1931^{29}$. Later on, the focus towards fermentation was shifted to A. terrus strains as reported by Nelson et al. ${ }^{30}$ and Eimhjellen et al. ${ }^{31}$ who reported on the effect of different substrates (including sugars and alcohols) on IA production. Initially industrial production of IA was performed via pyrolysis of citric acid to itaconic anhydride, followed by hydrolysis of anhydride ${ }^{28}$. Many other techniques are evaluated for the synthesis of the IA through genetic engineering of various strains for higher production of IA. Due to the high demand of biobased alternatives, IA has become a valuable compound in the biotechnological industry. To compete with the conventional chemical methods, biorefineries are taking up the challenge to cut down the production cost by producing IA on a larger scale ${ }^{32}$. Over the last decades, IA has been used for the synthesis of functional polyesters ${ }^{33,34,35,36,37,38,39}$, polyamides ${ }^{40,41,42,43}$ and other polymers ${ }^{44}$. Besides the renewable origin of IA, it holds an additional chemical advantage: IA contains an exo-vinyl group (unsaturated bond) along with two carboxylic acid moieties. Together, these functionalities allow IA to participate in Michael addition reactions ${ }^{45,46}$ with $\operatorname{amines}^{47}$, thiols ${ }^{38,48}$, or acetyl acetone ${ }^{49,50}$, 
thereby providing access to a broad range of IA derived compounds, usable for polymerization $^{51,18,52}$.

\subsection{Michael addition reaction}

The chemical reaction named as the Michael addition reaction was introduced in the year of 1884 by the research of Arthur Michael, inspired by the work of Conard and Kuthzeit ${ }^{53}$. Initially, the Michael addition was considered as the addition of an enolate ion into the $\beta$-carbon atom of an $\alpha, \beta$-unsaturated carbonyl compound ${ }^{53}$. Over the years, the Michael addition is not confined to the addition of only carbanions, as variety of nucleophiles bearing heteroatoms like nitrogen, oxygen, sulphur, and phosphor proved suitable Michael donors (scheme 1.1). Compounds bearing such electron rich heteroatoms, proved to act as nucleophile and actively participate in addition reaction to the $\mathrm{C}=\mathrm{C}$ (unsaturated) bond of Michael acceptor. Generally, this reaction is activated by a neighboring electron withdrawing group (EWG) located on the Michael acceptor, such as a carbonyl, nitrite or cyanide functionality. In contrast, the presence of electron donating groups on the Michael acceptor, the inductive effect $(+\mathrm{I}$ effect) or resonance stabilizing effect $(+\mathrm{R}$ effect) results in slow or no aza-Michael addition reaction due to the decrease in electrophilicity of the $\mathrm{C}=\mathrm{C}$ (unsaturation bond $)^{54,55}$. Nevertheless, the Michael addition reaction can be considered as a versatile tool for the formation of $\mathrm{C}-\mathrm{C}$, and $\mathrm{C}-\mathrm{X}(\mathrm{X}=\mathrm{N}, \mathrm{O}, \mathrm{P}, \mathrm{S})$ bonds in organic synthesis.

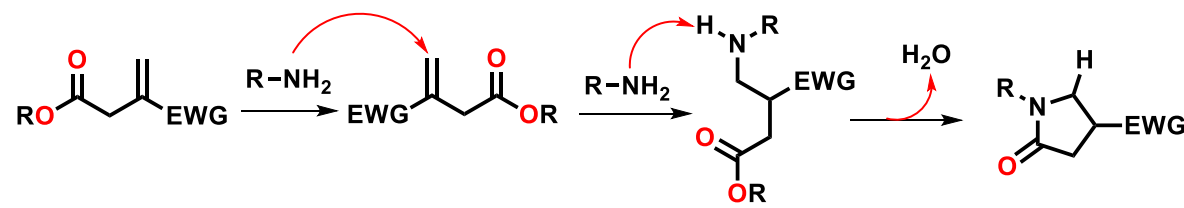

Scheme 1.1. Schematic diagram of the Michael addition reaction, while using itaconic acid as Michael acceptor and a nucleophilic alkyl group $\left(\mathrm{NH}_{2}-\mathrm{R}\right)$ as Michael donor.

\subsubsection{Aza-Michael addition/cyclization cascade reaction - Biomass feedstocks to biobased monomers}

Based on the nature of the nucleophile (Michael donor), Michael addition reaction is classified as carba-Michael addition, aza-Michael addition, oxa-Michael addition, phospho-Michael addition and thia-Michael addition reaction. The focus in this thesis is restricted to the used of the nitrogen nucleophiles only, hence on the azaMichael addition reaction ${ }^{56}$. It was firstly reported by McElvain et al. in 1948 who noted that the aza-Michael addition reaction is a second order reaction due to its 
dependency of rate co-efficient on both the concentration of the Michael acceptor and donor (amines in this case). Over the years, various catalysts have been reported for the aza-Michael addition reaction, including acid or base catalysts ${ }^{57,58}$, silica supported acids ${ }^{59,60}$, organic compounds $s^{61,62,63,64}$, clay supported Lewis acids ${ }^{65}$, organic polymers ${ }^{66}$, and ionic liquids ${ }^{67,68}$. Furthermore, the aza-Michael addition reaction can be conducted in water or ${ }^{69}$ and solvent free approach (bulk). ${ }^{70,57,71}$ During the course of this reaction, several factors such as reactivity of amines, nucleophilicity, and steric hindrance affect the reactivity of the Michael donor and determine the rate and progress of the reaction. ${ }^{72,73,74}$ An example, reacting aromatic amines with any $\alpha, \beta$ - unsaturated acrylate derivative requires harsh conditions (i.e. high temperatures) whereas reaction with aliphatic amines proceeds readily at room temperature. ${ }^{75}$ In particular, the aza-Michael addition on itaconic acid provides a toolbox of for the development of compounds having mono, di or multi-functional reactive groups usable for polymerization, as reported by groups of Sperry ${ }^{51}$, Miller $^{76,77}$, Wilsens ${ }^{78,79}$ and others ${ }^{40,51,45}$. Confining the attention towards developing the aza-Michael addition on itaconic acid, a consecutive cyclization cascade reaction has been reported already in 1950 by Paytash and his coworkers when using primary amines. ${ }^{80}$ While reacting a primary amine or ammonia with itaconic acid, having a carboxylic acid (or ester group, when using itaconic esters) situated on the $\gamma$-position unsaturated bond (acting as EWG group), it forms a secondary amine as aza-Michael adduct. In turn, this adduct undergoes an intra-molecular cyclization with the ester or acid group spaced at the $\gamma$-position, to form a stable 5-membered $\mathrm{N}$-substituted pyrrolidone ring (scheme 1.1, right). ${ }^{56,81}$ In this contrast, such a cascade reaction is not observed while using secondary amine as the Michael donor, thereby a tertiary amine is obtained as end Michael product. This aza-Michael addition/cyclization cascade reaction is reported for various other biobased monomers including fumarate and maleate esters, trans-aconitic and, and muconic acid provides a toolbox for the development of thermally stable and renewable monomers suitable for polymerization..$^{82,83}$

\subsubsection{Aza-Michael addition/cyclization cascade reaction - Biobased monomers into polymers}

Over the years, the aza-Michael addition reaction has been employed successfully for the synthesis of pyrrolidone based carboxylic acids, as reported by Miller et al. ${ }^{76,77,84,51}$, Wilsens et al. ${ }^{78}$, Ali et al. ${ }^{40,42}$, Malferrari et al. ${ }^{85}$ and Qi et al. ${ }^{76}$ In turn, these monomers have been used for the synthesis of polyesters, polyamides and poly(ester-amide)s. In addition, as reported by Ali and his coworkers ${ }^{40}$ and Wang and coworkers $^{86}$, the one pot reaction of itaconic acid and aliphatic diamines or aromatic diamines leads to either conventional poly-condensation reaction or salt formation 
and subsequent poly condensation yielding mono-pyrrolidone polyamides via azaMichael addition and cyclisation cascade reaction proceeding under same reaction conditions, yielding high $T_{\mathrm{g}}$, amorphous polyamides.

It is not surprising that aza-Michael addition is not restricted to small molecules; it can also be conducted on/for polymers as reported by groups of Pellis ${ }^{47}$, Chanda and Ramakrishnan ${ }^{50}$, and Farmer ${ }^{49,47}$ which used the aza-Michael addition reaction for polymer post-modification or post-polymerization steps while using the pendant functional groups (abbreviated as PFGs). The conventional polycondensation reaction of itaconic acid with various diols yields polyesters bearing having residual unsaturated exo-vinyl bond in the polymeric backbone available for post-polymerization modification (Scheme 1.2) ) $^{33,34,35}$. This functionality is still susceptible to undergo an aza-Michael addition, as reported by Robert and Friebel et al. ${ }^{36}$ However, the use of secondary amines are preferred as the aza-Michael adducts cannot undergo further cyclization reactions. Instead, the use of primary amines as reactants for post-functionalization of itaconic acid based polyesters is followed by the cascade cyclization reaction, resulting in chain scission of the polymers, as is reported by Farmer and coworkers ${ }^{47}$ (scheme 1.2, top). This azaMichael addition and cyclization reaction is irreversible in nature, unlike some minor reversibility observed at elevated temperature as reported by Pellis et al ${ }^{87}$.

Nevertheless, the employed post-functionalization is a well-known technique for synthesizing functional polymers with tunable properties like hydrophilicity, degradability and thermal properties. It is worth to note that itaconic acid undergoes isomerization on its exo-vinyl bond, and is known to be responsible for (undesired) crosslinking, converting the polyesters into thermosets. ${ }^{47}$ 


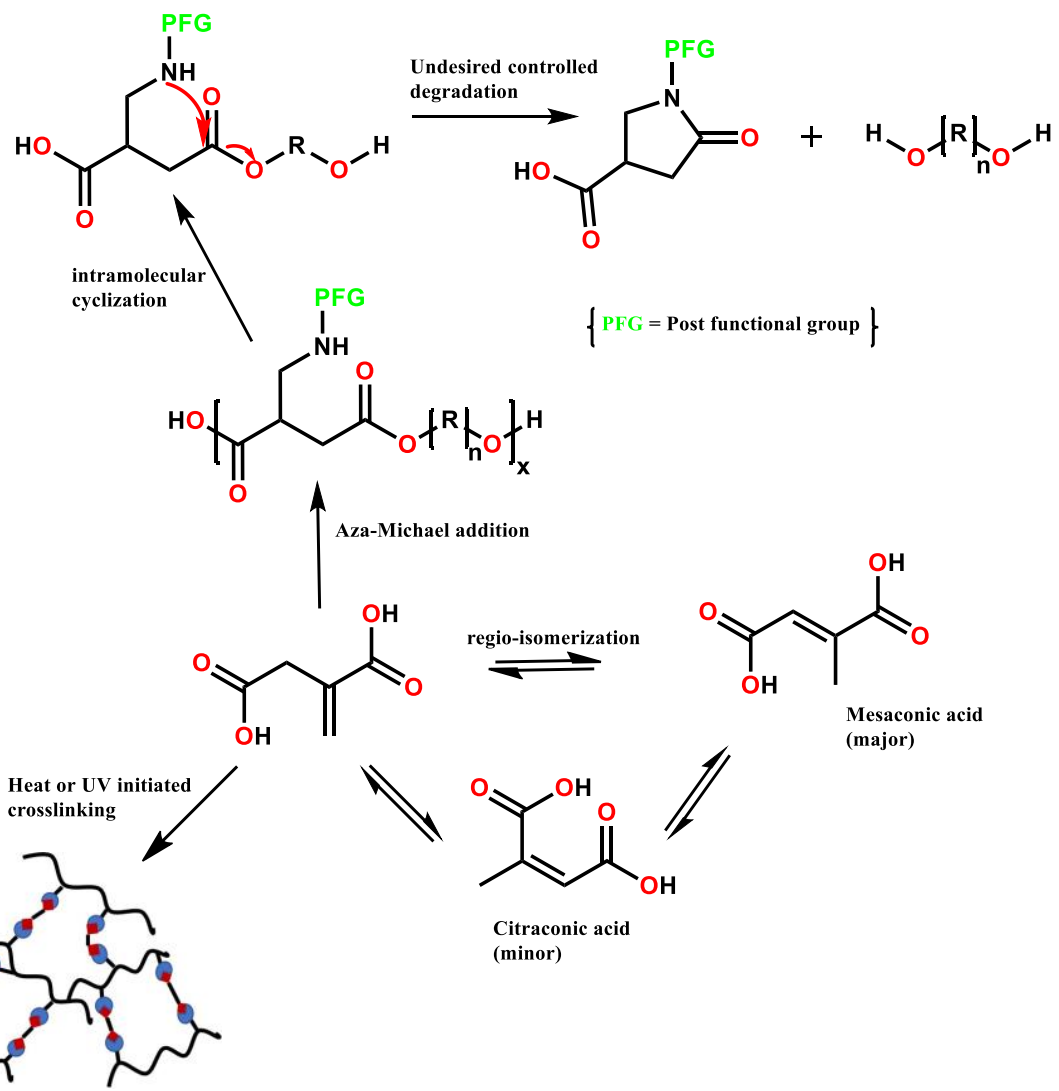

Scheme 1.2. A schematic overview showing the various region-isomers of itaconic acid, usage of exo-type double bond of itaconic acid with functionalized amines via aza-Michael addition/cyclization reaction resulting in thermal controlled degradation of the synthesized unsaturated polyester (UPE) via ring closure forming lactam ring and usage of exo-double bond for heat or UV initiated crosslinking.

\subsection{Oxazolines - A class of heterocyclic ring}

Oxazolines are introduced to the world of chemistry already early in the $19^{\text {th }}$ century by Wenker ${ }^{88}$, Fry ${ }^{89}$ and Goldberg ${ }^{90}$. The 2-oxazoline ring is a five membered heterocyclic ring, containing one oxygen and nitrogen atom and are widely employed as ligands in asymmetric catalysis and as protecting groups for carboxylic acids $^{91,92,93,94,95}$. These 2-oxazolines can be synthesized from carboxylic acids using amino alcohol ${ }^{96}$, haloalkylamides ${ }^{97}$, or from nitriles ${ }^{98,99}$ and aziridines ${ }^{100}$. There are various mechanisms proposed for the synthesis of oxazolines in literature; however, 
the synthesis of bis(2-oxazolines) using amino alcohol and dicarboxylic acid such as 2,5-furandicarboxylic acid is of particular interest due to their renewable origin. Various catalysts such as lanthanide chloride ${ }^{101}$, DAST $^{102,103}$, Deoxo-Fluor reagent ${ }^{102}$, 2-chloro-4,6-dimethoxy-1,3,5-triazine ${ }^{104}$, acidic zeolite ${ }^{105}$ and molybdenum oxide ${ }^{106}$ can be used as dehydrating catalysts for the generation of 2-oxazolines ${ }^{107}$. As an alternative route, the synthesis of bis(2-oxazolines) are described through conversion of dicarboxylic acids into acyl chlorides via treatment with thionyl chloride, followed with ring closure using chloroethylamine hydrochloride and ring closure in methanol containing sodium hydroxide. A third route involves the esterification of the dicarboxylic acid to methanol, followed by treating with ethanolamine and ring closure with thionyl chloride. However, following the green chemistry principles, the most sustainable and green synthetic pathway is to employ the reaction of $\beta$-amino alcohol with the dicarboxylic acids in refluxing condition, using toluene as solvent for 24 hours, with added molecular sieves or dehydrant (required for water deprivation and ring closure of oxazolines ring formation) ${ }^{108,109}$. An overview of the various synthesis routes obtaining bis(2-oxazolines) from dicarboxylic acids is shown in scheme 1.3 .

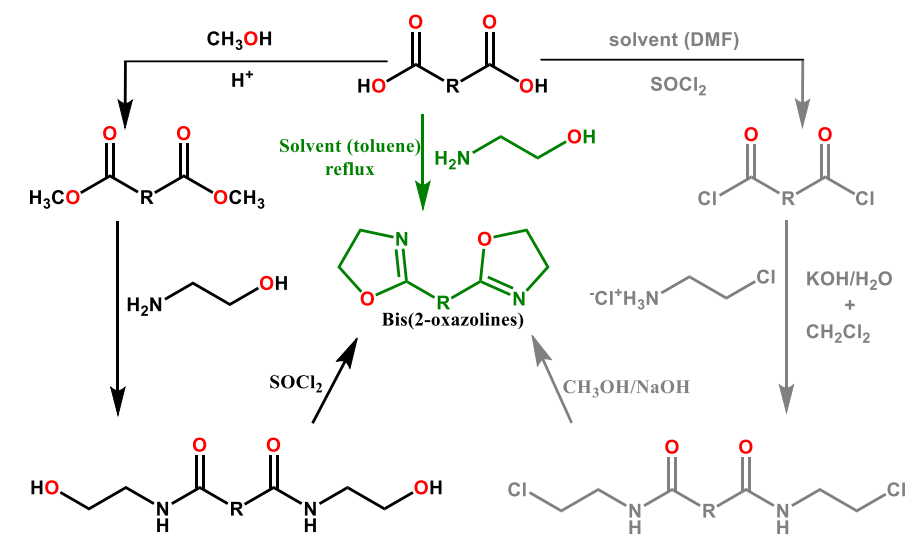

Scheme 1.3. Various reaction pathways to synthesize bis(oxazolines) starting from dicarboxylic acids, where $R=$ alkyl, phenyl, aromatic groups and so on.

\subsection{Renewable pyrrolidone monomers to polymers}

"Polymer" is now a days a word that encompasses our life starting from our pen to mobile phone, polymers are everywhere. Though polymers are generally obtained from fossil feedstock, endeavors of academia and industry have been undertaken to find a renewable alternative. Indeed, biomass such as lignin and cellulose proved to hold great potential as feedstock for the generation of renewable building blocks with various added functionality ${ }^{110,111,112,113}$. Furthermore, these 
biobased building blocks are interesting due to their additional functionality allowing for the generation of thermoplastics and thermosets.

\subsubsection{Thermoplastics}

Thermoplastics, as the name suggests, are a class of polymers, which can be molten and solidified reversibly upon heating. There are various conventional thermoplastics available including polyesters, polyamides and poly(ester-amides). In this respect, the pyrrolidone based dicarboxylic acid monomers have proven promising candidates for the development of biobased polymers. Generally, the reaction between pyrrolidone based carboxylic acids to a reaction with aliphatic alcohols, yields amorphous and degradable polyester material as reported by the groups of Miller 76,84 and others ${ }^{114,40,42}$. In contrast, the groups of Wilsens demonstrated the possibility for the development of semi-crystalline polymers from pyrrolidone based dicarboxylic acids ${ }^{78}$. In general, the presence of pyrrolidone rings is considered beneficial as it allows for the synthesis of high $T_{\mathrm{g}}$ materials; the pyrrolidone ring hinders the chain orientation due to its bulky chemical structure, thereby increasing the $T_{\mathrm{g}}$ compared to aliphatic fatty acids as reported by the groups of Wilsens ${ }^{78}$, Ali ${ }^{51}$ and Wang ${ }^{115}$. Furthermore, the potential of aza-Michael addition is extended towards developing functional renewable polyesters using naturally occurring $L$-amino acids with aliphatic side groups as is recently reported by the group of Miller ${ }^{84}$. In addition to the synthesis of polyesters, the pyrrolidone based dicarboxylic acids have been used for the generation of polyamides as reported by Noordzij and coworkers ${ }^{78}$, Wang and coworkers ${ }^{86}$, and others ${ }^{51,42,43}$. Hence, these pyrrolidone based carboxylic acids has proved its potential as a renewable building block together with its sustainable and green chemistry approach during its synthesis.

\subsubsection{Thermosetting resins}

Thermoset polymers are special class of polymers, which irreversibly solidify upon curing, initiated by heat, radiation, pressure, or catalyst. There are various conventional thermosets arising from covalently crosslinked networks available in the market like polyurethanes (via usage of isocyanides), epoxy, phenolformaldehydes, vinyl esters and unsaturated polyesters. Similarly, the use of bis(2oxazolines) has proven beneficial in developing poly(ester-amide)s, both thermoplastic and thermosetting resins, as reported by the groups of Hoogenboom ${ }^{116,117,118,119}$,Luxenhofer ${ }^{120,121}$, Nuyken ${ }^{122,123,124}$ and Schubert ${ }^{125,126,127,128}$ and Bohme $\mathrm{e}^{95,129,91}$.

Since last two centuries, thermal ring opening polymerization of 2oxazolines is employed for the generation of both thermoplastic and thermosetting polymers. Thermal polymerization proceeds via two pathways - one route is cationic 
ring opening polymerizations that yield poly (2-oxazolines), which has proven beneficial for biomedical applications, as is part of the above references by Hoogenboom, Nuyken, and Luxenhofer. Furthermore, groups of Sano ${ }^{130}$, Nery ${ }^{131}$, Wilsens ${ }^{108}$ reported on thermal ring opening polymerization with carboxylic acids; this reaction proceeds via abstraction of labile proton of the carboxylic acid group by the 2-oxazoline, yielding a carboxylate ion. This carboxylate ion now undergoes a nucleophilic attack on the $\mathrm{C} 5$ position of the positively charged 2-oxazoline ring, leading to the formation of an ester-amide (scheme 1.4). It may be no surprise that this thermal ring opening addition reaction of bis(2-oxazolines) have been widely used as chain extenders for carboxylic acid terminated polymers ${ }^{94,132}$. Examples include their use as chain extender for recycled PET or for the development of liquid crystalline polymers, contributing to the recycling of polymers or starting materials. Furthermore, 2-oxazolines have been reported to react with amines and alcohols; The reaction with amines proceeds at elevated temperatures generating amine-amide bonds, as reported by M.J.Fazio ${ }^{133}$. The reaction with aromatic phenols is reported by groups of Cuberston and Luston ${ }^{134}$, generating ether-amide bonds.

In addition to the linear chain extension reaction ( $k_{1}$, scheme 1.4), the generated secondary amide groups participate in the ring opening polymerization with the 2-oxazoline moieties, thereby generating tertiary amide bonds $\left(k_{2}\right.$, scheme 1.4). In this regard, the formation of the tertiary amide bonds are responsible for crosslinking of the 2-oxazoline resins, where the crosslinking density can be controlled by using excess of bis(2-oxazolines) monomers. ${ }^{130,108}$

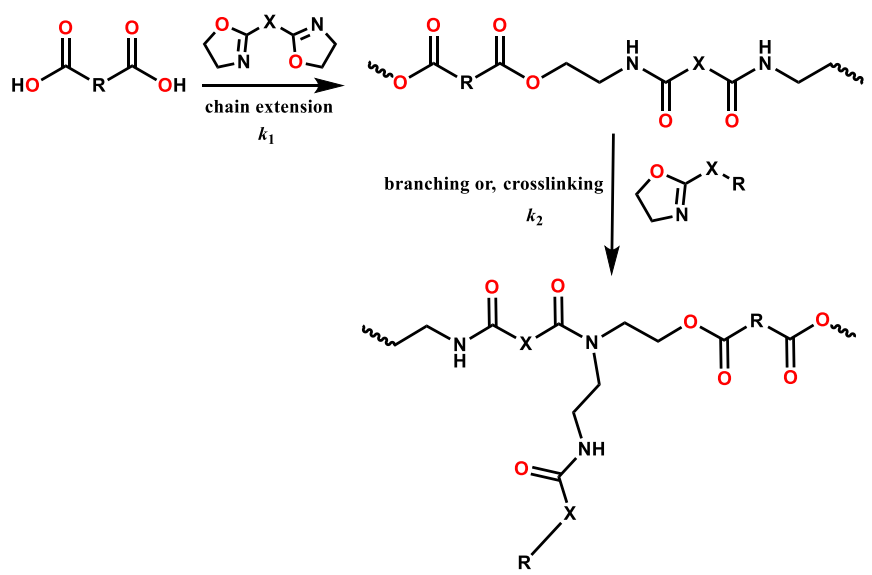

Scheme 1.4. Chain extension $\left(k_{1}\right)$ and branching $\left(k_{2}\right)$ reactions occurring during curing of the resin containing dicarboxylic acid and bis(2-oxazolines). The branching and crosslinking step is controlled with the excess amount of the used bis(2oxazolines). 


\subsection{Reversible dynamic networks (Recyclable thermosets)}

It is noted that the chemical resistance of the thermosets is often considered excellent; these covalently bonded thermosets suffer from a poor recyclability. To this end, dynamic or reversible network has gained a lot of both academic and industrial attention. Reversible network formation in practice, uses chemistry like Diels Alder and Retro Diels Alder reaction ${ }^{135,136,137}$ for the formation of reversible and thermo-responsive covalent networks. Similarly, hydrogen bonding ${ }^{138,139,140}$, ionic bonding and ionomers ${ }^{141,142}$ are also used to generate polymers having physical networks. The association and dissociation kinetics of these reactive species are responsible for their smart properties like repairing or self-healing used under optimum conditions. The self-healing mechanism was introduced first by Ludwik Leibler, which led to a new generation of thermosets that are repairable, healable or reprocessable after damage or fracture ${ }^{143,144,145,146,147}$. This class of thermosets outshined traditional thermosets in terms of sustainability, moreover, provided a platform for the development of this new generation of thermosets. This class of reprocessable thermosets have evolved over the years while exploring various possible chemistry leading to dynamic covalent crosslinking, as shown by F.E.Du.Prez et al. ${ }^{148-155}$, C.N.Bowman et al. ${ }^{156-162}$, and others ${ }^{173-177}$. In case of reversible networks, the presence of non-permanent covalent networks or crosslinks can break and make through reversible chemical bonding. As reported by F.E.D.Prez et al. ${ }^{151}$, the reversible polymer networks undergoes topological changes due to the fluctuations or dissolution of the overall connectivity during heating, thereby undergoing deformation or melting of the polymer. Furthermore, upon cooling down (below the glass transition temperature) the original state of the polymer is recovered (scheme 1.5). Please note, the behavior of this class of networks is different from that of vitrimers, where the fluctuations occur although the number of connectivity remains the same throughout the reaction time. Thus, these materials (reversible networks) behave both as thermoplastic and thermosets depending on the conditions applied. As for example, heat may trigger the loss of connectivity in a reversible dynamic network. Similarly does the aprotic polar solvents like dimethyl sulphoxide, dimethyl formamide, can also readily trigger the loss of connectivity and hence complete dissolution of the crosslinked polymer is facilitated. Thus, the reversible dynamic networks might be molten or solubilized and hence can be reprocessed with ease under any of these conditions (of choice). 

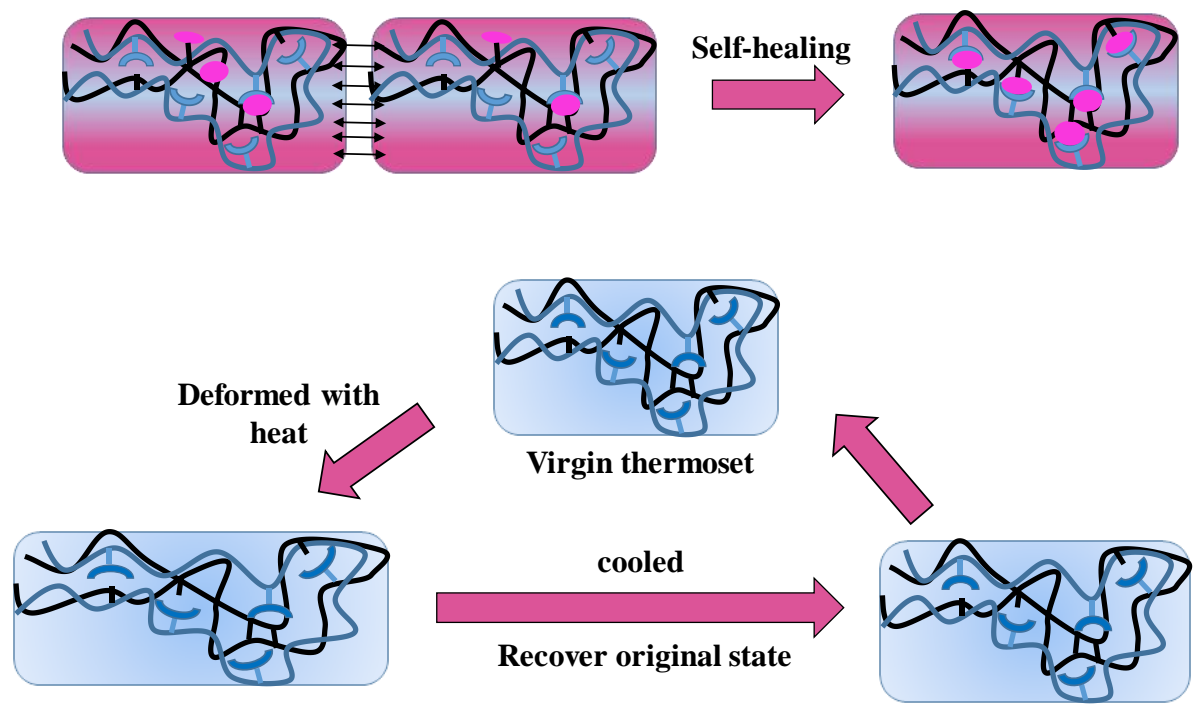

Scheme 1.5: Plausible mechanism of reversible dynamic bonding in polymers, leading to reusage, recyclable and self-healable polymers via physical attractions or reversible chemical bonding triggered with heat.

\subsection{End of life}

Most of the traditional thermosets, based on for example epoxy or polyurethane, suffer from limitations in recyclability resulting from the presence of covalent crosslinks ${ }^{168,169}$. In contrast, thermosets based on polyester, polyamide, or poly(ester-amide)s proved to hold more promise with respect to their end-of-lie possibilities as they can be depolymerized via hydrolysis ${ }^{170,171}$ or enzymatic depolymerization ${ }^{172}$, can be subjected to biodegradation ${ }^{173,42}$ and can be chemically recycled ${ }^{168,174}$. As mentioned earlier, bis(2-oxazolines) derived polymers proved useful for the synthesis of polyamides for biomedical applications ${ }^{175,176,177,178,179}$ and poly(ester-amide)s ${ }^{108,180,95,129}$ for various thermoplastic and thermosetting applications. These polymers, are prone to both hydrolysis and biodegradation via enzymes or bacteria ${ }^{173,42,42,171,181,182,183,184}$. As an end of life, enzymatic biodegradation may be the safest option; if ended as carbon dioxide and water. In some cases, biodegradation (when left in soil) yields small oligomers, which remains soluble in water thereby harming the aquatic life ecosystem. Perhaps a more sustainable approach would involve chemical recycling or enzymatic depolymerization. In this case, the polymer is depolymerized, and either the monomers or the starting materials can be isolated and reused. 


\subsection{Aim and scope of the thesis}

The main motivation of this work is to evaluate the potential of polymers obtained from bis(2-oxazolines) and renewable pyrrolidone based dicarboxylic acids. This project is partly funded by Interegg Vlaanderen-Nederland and is a part of the BB100 project. The aim of this project is to develop fully renewable fibers, including all additives. The work performed in this thesis contributed to the development of novel monomers usable for curing with 2-oxazolines into free-standing films or adhesives, and for the development of nucleating agents using the same chemistry. To this end, monomers are synthesized from, amongst others, the renewable itaconic acid, 2,5-furandicarboxylic acid, and various naturally occurring L-amino acids. The kinetics of the thermal ring-opening addition reaction between the bis(2-oxazolines) and the developed dicarboxylic acids is studied, the mechanical performance and adhesion of the ensuing polymers is evaluated, and their potential for enzymatic depolymerization and chemical recycling of the monomers is identified (scheme 1.6).

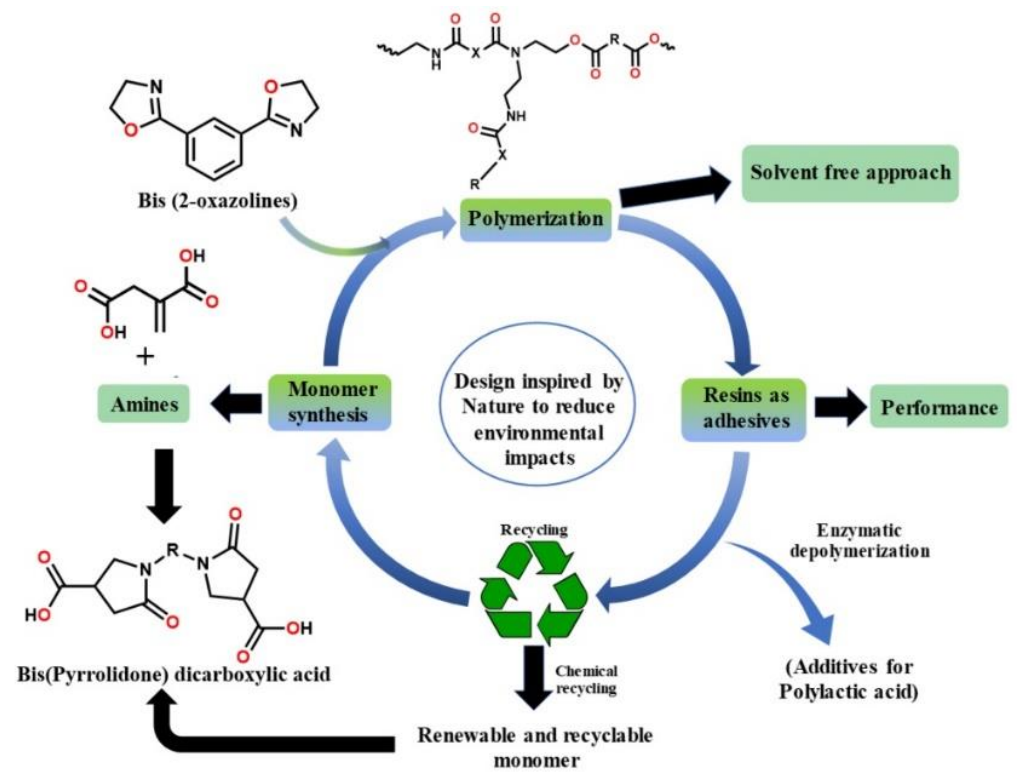

Scheme 1.6: Schematic representation showing summary of the thesis starting with synthesis of biobased monomers and its utilization in developing biobased resins, followed with evaluation of its mechanical performances and formation of dynamic networks based on 2-oxazolines chemistry. Lastly, their end of life is predicted via various pathways like enzymatic depolymerization and chemical recycling and the recovered organic compounds are further reused for polymerizations with 2oxazolines and as additives and adhesives for PLA. 
In more detail; In Chapter 2, the synthesis of a series of asymmetric pyrrolidone dicarboxylic acids from itaconic acid and aliphatic diamines is reported. This chapter details about the employed reaction conditions, analysis, yield, and thermal stability of the developed monomers. In the second part we evaluate the potential of these monomers in the thermal curing with bis(2-oxazolines). It is noteworthy that these monomers exhibit a significantly increased reactivity towards bis(2-oxazolines) compared to regular aliphatic dicarboxylic acids (sebacic acid), allows for shorter curing times. Lastly, the possibility for enzymatic depolymerization of the developed materials is explored.

The development of thermosets using the thermal ring-opening addition reaction in bis(2-oxazolines) resins is explored in Chapter 3. The developed thermosets proved highly sensitive to absorb water, resulting in a plasticizing effect and facilitating a brittle-to-ductile transition in their mechanical performance. The thermo-mechanical behavior in the presence of various amounts of water is discussed in detail and the adhesive properties of the developed polymers on alumina and polylactide are explored. Lastly, the possibility for chemical recycling of the (bis)pyrrolidone monomer, after hydrolysis of the developed polymers, is evaluated.

In Chapter 4, the synthesis of multifunctional pyrrolidone based dicarboxylic acids from itaconic acid and naturally occurring L-amino acids is reported. Examples of monomers that are used include $L$-phenyl alanine, $L$-tyrosine and $L$-histidine. The curing of these monomers with bis(2-oxazolines) is performed with the aim to generate polymers bearing pendant functional groups existing in the employed amino acid. For example, the polymerization of a pyrrolidine based dicarboxylic acid derived from L-tyrosine with an isophthalic acid based bis(2oxazolines) is performed, yielding a polymer bearing pendant phenol moieties. The visco-elastic and stress relaxation behavior are evaluated as a function of the chemical composition of the polymers and in the presence of pendant functional groups.

Chapter 5 shows the potential application the N,N'-bis(2hydroxyethyl)furan-2,5-dicarboxamide (BHEF) and N,N'-bis(2hydroxyethyl)terephthalamide (BHET) as additive for PLA. Both BHET and BHEF compounds are the hydrolyzed residues of polymers based on 2,5-furandicarboxylic acid or terephthalic acid based bis(2-oxazolines). In this chapter we evaluate their potential as plasticizer and nucleating agent for PLA. Though BHEF is renewable in origin, it proved to hold no interesting properties as additive in PLA. Instead, BHET proved to function both as a plasticizer and nucleating agent for PLA. Though the terephthalic acid from BHET is not renewable in origin, it can be isolated from recycling streams such as PET. 
Chapter 6 elaborates on a series of renewable oxalamide based nucleating agent for polylactide (PLA), providing an alternative to the BHET reported in Chapter 4. The nucleation mechanism is investigated in detail using polarized optical microscopy, DSC, and rheological analysis. Overall, we observed that the enhancement in PLA nucleation in the presence of these oxalamide based nucleating agents does not originate from epitaxy or soft-epitaxy but instead results from surface roughness and stress-enhanced nucleation. More importantly, the performance of these nucleating agents improves when subjected to increasing cooling rates, making these interesting for use under industrial processing conditions.

Lastly, the conclusion, outlook, recommendation and valorization of this thesis are given in Chapter 7. 


\subsection{References}

(1) Sauvé, S.; Bernard, S.; Sloan, P. Environmental Sciences, Sustainable Development and Circular Economy: Alternative Concepts for Trans-Disciplinary Research. Environ. Dev. 2016, 17, 48-56. https://doi.org/10.1016/j.envdev.2015.09.002.

(2) Houshyar, A. N.; Hoshyar, A. N.; Sulaiman, R. Review Paper on Sustainability in Manufacturing System. J. Appl. Environ. Biol. Sci. 2014, 4 (4), 7-11.

(3) Suárez-Eiroa, B.; Fernández, E.; Méndez-Martínez, G.; Soto-Oñate, D. Operational Principles of Circular Economy for Sustainable Development: Linking Theory and Practice. J. Clean. Prod. 2019, 214, 952-961. https://doi.org/10.1016/j.jclepro.2018.12.271.

(4) Kirchherr, J.; Piscicelli, L. Towards an Education for the Circular Economy (ECE): Five Teaching Principles and a Case Study. Resour. Conserv. Recycl. 2019, 150 (July), 104406. https://doi.org/10.1016/j.resconrec.2019.104406.

(5) Jawahir, I. S.; Bradley, R. Technological Elements of Circular Economy and the Principles of 6R-Based Closed-Loop Material Flow in Sustainable Manufacturing. Procedia CIRP 2016, 40, 103-108. https://doi.org/10.1016/j.procir.2016.01.067.

(6) Kalmykova, Y.; Sadagopan, M.; Rosado, L. Circular Economy - From Review of Theories and Practices to Development of Implementation Tools. Resour. Conserv. Recycl. 2018, 135 (February 2017), 190-201. https://doi.org/10.1016/j.resconrec.2017.10.034.

(7) Jawahir, I. S.; Badurdeen, F.; Rouch, K. E. Innovation in Sustainable Manufacturing Education. 11th Glob. Conf. Sustain. Manuf. 2013, 9-16.

(8) Adams, K. T.; Osmani, M.; Thorpe, T.; Thornback, J. Circular Economy in Construction: Current Awareness, Challenges and Enablers. Proc. Inst. Civ. Eng. Waste Resour. Manag. 2017, 170 (1), 15-24. https://doi.org/10.1680/jwarm.16.00011.

(9) Moreau, V.; Sahakian, M.; van Griethuysen, P.; Vuille, F. Coming Full Circle: Why Social and Institutional Dimensions Matter for the Circular Economy. J. Ind. Ecol. 2017, 21 (3), 497-506. https://doi.org/10.1111/jiec.12598.

(10) Prieto-Sandoval, V.; Jaca, C.; Ormazabal, M. Towards a Consensus on the Circular Economy. J. Clean. Prod. 2018, 179, 605-615. https://doi.org/10.1016/j.jclepro.2017.12.224.

(11) Sheldon, R. A. Metrics of Green Chemistry and Sustainability: Past, Present, and Future. ACS Sustain. Chem. Eng. 2018, 6 (1), 32-48. https://doi.org/10.1021/acssuschemeng.7b03505.

(12) Karpudewan, M.; Ismail, Z.; Roth, W. M. Ensuring Sustainability of Tomorrow through Green Chemistry Integrated with Sustainable Development Concepts (SDCs). Chem. Educ. Res. Pract. 2012, 13 (2), 120-127. https://doi.org/10.1039/c1rp90066h.

(13) Anastas, P. T.; Warner, J. C. Green Chemistry: Theory and Practice; Oxford University Press: Oxford [England]; New York, 1998.

(14) Sheldon, R. A. Fundamentals of Green Chemistry: Efficiency in Reaction Design. Chem. Soc. Rev. 2012, 41 (4), 1437-1451. https://doi.org/10.1039/c1cs15219j.

(15) Sheldon, R. A. Consider the Environmental Quotient. CHEMTECH;(United States) 1994, 24 
(3).

(16) Sheldon, R. A. Green Solvents for Sustainable Organic Synthesis: State of the Art. Green Chem. 2005, 7 (5), 267-278. https://doi.org/10.1039/b418069k.

(17) Horváth, I. T.; Anastas, P. T. Innovations and Green Chemistry. Chem. Rev. 2007, 107 (6), 2169-2173. https://doi.org/10.1021/cr078380v.

(18) Medway, A. M.; Sperry, J. Heterocycle Construction Using the Biomass-Derived Building Block Itaconic Acid. Green Chem. 2014, 16 (4), 2084-2101.

https://doi.org/10.1039/c4gc00014e.

(19) Lebarbé, T.; Maisonneuve, L.; Nga Nguyen, T. H.; Gadenne, B.; Alfos, C.; Cramail, H. Methyl 10-Undecenoate as a Raw Material for the Synthesis of Renewable Semi-Crystalline Polyesters and Poly(Ester-Amide)S. Polym. Chem. 2012, 3 (10), 2842-2851.

https://doi.org/10.1039/c2py20394d.

(20) Wang, Z.; Yuan, L.; Tang, C. Sustainable Elastomers from Renewable Biomass. Acc. Chem. Res. 2017, 50 (7), 1762-1773. https://doi.org/10.1021/acs.accounts.7b00209.

(21) Pfaltzgraff, L. A.; De Bruyn, M.; Cooper, E. C.; Budarin, V.; Clark, J. H. Food Waste Biomass: A Resource for High-Value Chemicals. Green Chem. 2013, 15 (2), 307-314. https://doi.org/10.1039/c2gc36978h.

(22) Teleky, B. E.; Vodnar, D. C. Biomass-Derived Production of Itaconic Acid as a Building Block in Specialty Polymers. Polymers (Basel). 2019, 11 (6).

https://doi.org/10.3390/polym11061035.

(23) Bohre, A.; Novak, U.; Grilc, M.; Likozar, B. Synthesis of Bio-Based Methacrylic Acid from Biomass-Derived Itaconic Acid over Barium Hexa-Aluminate Catalyst by Selective Decarboxylation Reaction. Mol. Catal. 2019, 476 (February).

https://doi.org/10.1016/j.mcat.2019.110520.

(24) Pedroso, G. B.; Montipó, S.; Mario, D. A. N.; Alves, S. H.; Martins, A. F. Building Block Itaconic Acid from Left-over Biomass. Biomass Convers. Biorefinery 2017, 7 (1), 23-35. https://doi.org/10.1007/s13399-016-0210-1.

(25) Klement, T.; Büchs, J. Itaconic Acid - A Biotechnological Process in Change. Bioresour. Technol. 2013, 135, 422-431. https://doi.org/10.1016/j.biortech.2012.11.141.

(26) Klement, T.; Milker, S.; Jäger, G.; Grande, P. M.; Domínguez de María, P.; Büchs, J. Biomass Pretreatment Affects Ustilago Maydis in Producing Itaconic Acid. Microb. Cell Fact. 2012, 11, 1-13. https://doi.org/10.1186/1475-2859-11-43.

(27) Jiménez-Quero, A.; Pollet, E.; Zhao, M.; Marchioni, E.; Avérous, L.; Phalip, V. Itaconic and Fumaric Acid Production from Biomass Hydrolysates by Aspergillus Strains. J. Microbiol. Biotechnol. 2016, 26 (9), 1557-1565. https://doi.org/10.4014/jmb.1603.03073.

(28) Baup, S. Ueber Eine Neue Pyrogen-Citronensäure, Und Über Benennung Der Pyrogen-Säuren Überhaupt. Ann. der Pharm. 1836, 19 (1), 29-38. https://doi.org/10.1002/jlac.18360190107.

(29) Steiger, M. G.; Blumhoff, M. L.; Mattanovich, D.; Sauer, M. Biochemistry of Microbial Itaconic Acid Production. Front. Microbiol. 2013, 4, 271-287.

https://doi.org/10.3389/fmicb.2013.00023. 
(30) Nelson, G. E. N.; Traufler, D. H.; Kelley, S. E.; Lockwood, L. B. Production of Itaconic Acid by Aspergillus Terreus in 20-Liter Fermentors. Ind. Eng. Chem. 1952, 44 (5), 1166-1168. https://doi.org/10.1021/ie50509a062.

(31) Eimhjellen, K. E.; LARSEN, H. The Mechanism of Itaconic Acid Formation by Aspergillus Terreus. 2. The Effect of Substrates and Inhibitors. Biochem. J. 1955, 60 (1), 139. https://doi.org/10.1042/bj0600139.

(32) Bozell, J. J.; Petersen, G. R. Technology Development for the Production of Biobased Products from Biorefinery Carbohydrates - the US Department of Energy's “Top 10" Revisited. Green Chem. 2010, 12 (4), 539-554.

(33) Dai, J.; Ma, S.; Liu, X.; Han, L.; Wu, Y.; Dai, X.; Zhu, J. Synthesis of Bio-Based Unsaturated Polyester Resins and Their Application in Waterborne UV-Curable Coatings. Prog. Org. Coatings 2015, 78, 49-54. https://doi.org/10.1016/j.porgcoat.2014.10.007.

(34) Barrett, D. G.; Merkel, T. J.; Luft, J. C.; Yousaf, M. N. One-Step Syntheses of Photocurable Polyesters Based on a Renewable Resource. Macromolecules 2010, 43 (23), 9660-9667. https://doi.org/10.1021/ma1015424.

(35) Panic, V. V.; Seslija, S. I.; Popovic, I. G.; Spasojevic, V. D.; Popovic, A. R.; Nikolic, V. B.; Spasojevic, P. M. Simple One-Pot Synthesis of Fully Biobased Unsaturated Polyester Resins Based on Itaconic Acid. Biomacromolecules 2017, 18 (12), 3881-3891.

https://doi.org/10.1021/acs.biomac.7b00840.

(36) Robert, T.; Friebel, S. Itaconic Acid-a Versatile Building Block for Renewable Polyesters with Enhanced Functionality. Green Chem. 2016, 18 (10), 2922-2934.

https://doi.org/10.1039/c6gc00605a.

(37) Brännström, S.; Malmström, E.; Johansson, M. Biobased UV-Curable Coatings Based on Itaconic Acid. J. Coatings Technol. Res. 2017, 14 (4), 851-861.

https://doi.org/10.1007/s11998-017-9949-y.

(38) Lv, A.; Cui, Y.; Du, F. S.; Li, Z. C. Thermally Degradable Polyesters with Tunable Degradation Temperatures via Postpolymerization Modification and Intramolecular Cyclization. Macromolecules 2016, 49 (22), 8449-8458.

https://doi.org/10.1021/acs.macromol.6b01325.

(39) Lv, A.; Li, Z. L.; Du, F. S.; Li, Z. C. Synthesis, Functionalization, and Controlled Degradation of High Molecular Weight Polyester from Itaconic Acid via ADMET Polymerization. Macromolecules 2014, 47 (22), 7707-7716. https://doi.org/10.1021/ma5020066.

(40) Ali, M. A.; Tateyama, S.; Oka, Y.; Kaneko, D.; Okajima, M. K.; Kaneko, T. Syntheses of High-Performance Biopolyamides Derived from Itaconic Acid and Their Environmental Corrosion. Macromolecules 2013, 46 (10), 3719-3725. https://doi.org/10.1021/ma400395b.

(41) Ayadi, F.; Mamzed, S.; Portella, C.; Dole, P. Synthesis of Bis(Pyrrolidone-4-Carboxylic Acid)Based Polyamides Derived from Renewable Itaconic Acid - Application as a Compatibilizer in Biopolymer Blends. Polym. J. 2013, 45 (10), 766-774. https://doi.org/10.1038/pj.2012.206.

(42) Ali, M. A.; Tateyama, S.; Kaneko, T. Syntheses of Rigid-Rod but Degradable Biopolyamides from Itaconic Acid with Aromatic Diamines. Polym. Degrad. Stab. 2014, 109 (July), 367-372. https://doi.org/10.1016/j.polymdegradstab.2014.05.031. 
(43) He, M.; Wang, Z.; Wang, R.; Zhang, L.; Jia, Q. Preparation of Bio-Based Polyamide Elastomer by Using Green Plasticizers. 2016, 86-10. https://doi.org/10.3390/polym8070257.

(44) Lv, A.; Li, Z.-L. Z.-C.; Du, F.-S.; Li, Z.-L. Z.-C. Synthesis, Functionalization, and Controlled Degradation of High Molecular Weight Polyester from Itaconic Acid via ADMET Polymerization. Macromolecules 2014, 47 (22), 7707-7716.

https://doi.org/10.1021/ma5020066.

(45) Guarneri, A.; Cutifani, V.; Cespugli, M.; Pellis, A.; Vassallo, R.; Asaro, F.; Ebert, C.;

Gardossi, L. Functionalization of Enzymatically Synthesized Rigid Poly(Itaconate)s via PostPolymerization Aza-Michael Addition of Primary Amines. Adv. Synth. Catal. 2019, 361 (11), 2559-2573. https://doi.org/10.1002/adsc.201900055.

(46) Bosica, G.; Spiteri, J.; Borg, C. Aza-Michael Reaction: Selective Mono- versus Bis-Addition under Environmentally-Friendly Conditions. Tetrahedron 2014.

https://doi.org/10.1016/j.tet.2014.02.021.

(47) Farmer, T. J.; Comerford, J. W.; Pellis, A.; Robert, T. Post-Polymerization Modification of Bio-Based Polymers: Maximizing the High Functionality of Polymers Derived from Biomass. Polym. Int. 2018, 67 (7), 775-789. https://doi.org/10.1002/pi.5573.

(48) Winkler, M.; Lacerda, T. M.; Mack, F.; Meier, M. A. R. R. Renewable Polymers from Itaconic Acid by Polycondensation and Ring-Opening-Metathesis Polymerization. Macromolecules 2015, 48 (5), 1398-1403. https://doi.org/10.1021/acs.macromol.5b00052.

(49) Farmer, T. J.; Castle, R. L.; Clark, J. H.; Macquarrie, D. J. Synthesis of Unsaturated Polyester Resins from Various Bio-Derived Platform Molecules. Int. J. Mol. Sci. 2015. https://doi.org/10.3390/ijms160714912.

(50) Chanda, S.; Ramakrishnan, S. Poly(Alkylene Itaconate)s - An Interesting Class of Polyesters with Periodically Located Exo-Chain Double Bonds Susceptible to Michael Addition. Polym. Chem. 2015, 6 (11), 2108-2114. https://doi.org/10.1039/c4py01613k.

(51) Saidi, M. R.; Pourshojaei, Y.; Aryanasab, F.; Ali, M. A.; Tateyama, S.; Oka, Y.; Kaneko, D.; Okajima, M. K.; Kaneko, T.; Qi, P.; et al. Synthesis of Biorenewable and Water---Degradable Polylactam Esters from Itaconic Acid. Tetrahedron Lett. 2007, 64 (40), 9540-9543. https://doi.org/10.1016/j.ultsonch.2011.11.009.

(52) Robert, T.; Friebel, S. Itaconic Acid-a Versatile Building Block for Renewable Polyesters with Enhanced Functionality. Green Chem. 2016, 18 (10), 2922-2934.

https://doi.org/10.1039/c6gc00605a.

(53) Tokoroyama, T. Discovery of the Michael Reaction. 2010, 2009-2016. https://doi.org/10.1002/ejoc.200901130.

(54) Genest, A.; Portinha, D.; Fleury, E.; Ganachaud, F. The Aza-Michael Reaction as an Alternative Strategy to Generate Advanced Silicon-Based (Macro)Molecules and Materials. Prog. Polym. Sci. 2017, 72, 61-110. https://doi.org/10.1016/j.progpolymsci.2017.02.002.

(55) Critchfield, F. E.; Funk, G. L.; Johnson, J. B. Determination of Alpha, Beta-Unsaturated Compouds by Reaction with Morpholine. Anal. Chem. 1956, 28 (1), 76-79. https://doi.org/10.1021/ac60109a024. 
Alternative Strategy to Generate Advanced Silicon-Based (Macro) Molecules and Materials. Prog. Polym. Sci. 2017, 72, 61-110.

(57) Wang, Y.; Yuan, Y.; Guo, S. Silica Sulfuric Acid Promotes Aza-Michael Addition Reactions under Solvent-Free Condition as a Heterogeneous and Reusable Catalyst. 2009, No. Iv, 47794789. https://doi.org/10.3390/molecules14114779.

(58) Bosica, G.; Abdilla, R. Aza-Michael Mono-Addition Using Acidic Alumina under Solventless Conditions. Molecules 2016, 21 (6). https://doi.org/10.3390/molecules21060815.

(59) You, L.; Feng, S.; An, R.; Wang, X.; Bai, D. Silica Gel Accelerated Aza-Michael Addition of Amines to a , b -Unsaturated Amides. 2008, 49, 5147-5149.

https://doi.org/10.1016/j.tetlet.2008.06.099.

(60) Sharma, G.; Kumar, R.; Chakraborti, A. K. Fluoroboric Acid Adsorbed on Silica-Gel ( HBF 4 - $\mathrm{SiO} 2$ ) as a New, Highly Efficient and Reusable Heterogeneous Catalyst for Thia-Michael Addition to a , b -Unsaturated Carbonyl Compounds. 2008, 49, 4272-4275.

https://doi.org/10.1016/j.tetlet.2008.04.144.

(61) Surendra, K.; Krishnaveni, N. S.; Sridhar, R.; Rao, K. R. ????-Cyclodextrin Promoted AzaMichael Addition of Amines to Conjugated Alkenes in Water. Tetrahedron Lett. 2006. https://doi.org/10.1016/j.tetlet.2006.01.124.

(62) Cai, Y.; Li, L. I.; Luo, M.; Yang, K.; Lai, G.; Jiang, J.; Xu, L. Organocatalytic Aza-Michael / Retro-Aza-Michael Reaction : Pronounced Chirality Amplification in Aza-Michael Reaction and Racemization via Retro-Aza-Michael Reaction. 2011, 403 (March 2010), 397-403. https://doi.org/10.1002/chir.

(63) Ying, A.; Li, Z.; Yang, J.; Liu, S.; Xu, S.; Yan, H.; Wu, C. DABCO-Based Ionic Liquids: Recyclable Catalysts for Aza-Michael Addition of $\alpha, \beta$-Unsaturated Amides under SolventFree Conditions. 2014. https://doi.org/10.1021/jo500937a.

(64) Kai, N. A. N-Methylimidazole as a Promising Catalyst for the Aza-Michael Addition Reaction of N-Heterocycles. 2007, 3-9. https://doi.org/10.1055/s-2007-983816.

(65) Mokhtar, M.; Saleh, T. S.; Basahel, S. N. Journal of Molecular Catalysis A : Chemical Mg - Al Hydrotalcites as Efficient Catalysts for Aza-Michael Addition Reaction : A Green Protocol.

"Journal Mol. Catal. A, Chem. 2012, 353-354, 122-131. https://doi.org/10.1016/j.molcata.2011.11.015.

(66) Dhake, K. P.; Tambade, P. J.; Singhal, R. S.; Bhanage, B. M. Promiscuous Candida Antarctica Lipase B-Catalyzed Synthesis of b -Amino Esters via Aza-Michael Addition of Amines to Acrylates. Tetrahedron Lett. 2010, 51 (33), 4455-4458.

https://doi.org/10.1016/j.tetlet.2010.06.089.

(67) Dewan, M.; De, A.; Mozumdar, S. Ef Fi Cient and Reusable Ionic Liquid Stabilized Magnetic Cobalt Nanoparticles as Catalysts for Aza- and Thia-Michael Reactions. INOCHE 2015, 53, 92-96. https://doi.org/10.1016/j.inoche.2015.01.027.

(68) Xu, L.; Li, J.; Zhou, S.; Xia, C.; Oxidation, S. A Green, Ionic Liquid and Quaternary Ammonium Salt-Catalyzed Aza-Michael Reaction of a , b -Ethylenic Compounds with Amines in Water. 2004, 183-184.

(69) Ranu, B. C.; Banerjee, S. Significant Rate Acceleration of the Aza-Michael Reaction in Water. 
Tetrahedron Lett. 2007. https://doi.org/10.1016/j.tetlet.2006.10.142.

(70) Ying, A.; Zheng, M.; Xu, H.; Qiu, F.; Ge, C. For Aza-Michael Addition under Solvent-Free Conditions. 2011, No. 3, 883-890. https://doi.org/10.1007/s11164-011-0296-9.

(71) Chen, X.; Li, X.; Song, H.; Qian, Y.; Wang, F. Solvent-Free Aza-Markovnikov and AzaMichael Additions Promoted by a Catalytic Amount of Imidazolide Basic Ionic Liquids. Tetrahedron Lett. 2011, 52 (28), 3588-3591. https://doi.org/10.1016/j.tetlet.2011.04.117.

(72) Matveeva, E. V; Petrovskii, P. V; Odinets, I. L. Efficient Synthesis of Racemic $\beta$ Aminophosphonates via Aza-Michael Reaction in Water. Tetrahedron Lett. 2008, 49 (42), 6129-6133. https://doi.org/10.1016/j.tetlet.2008.08.015.

(73) ZOU, B.; JIANG, H.-F. Synthesis of $\beta$-Amino Acids via Catalyst- and Solvent-Free AzaMichael Reaction. Chinese J. Chem. 2008, 26 (7), 1309-1314. https://doi.org/10.1002/cjoc.200890238.

(74) Wu, D.; Liu, Y.; He, C.; Chung, T.; Goh, S. Effects of Chemistries of Trifunctional Amines on Mechanisms of Michael Addition Polymerizations with Diacrylates. Macromolecules 2004, 37 (18), 6763-6770. https://doi.org/10.1021/ma0493832.

(75) Rulev, A. Y. Aza-Michael Reaction: Achievements and Prospects. Russ. Chem. Rev. 2011, 80 (3), 197-218. https://doi.org/10.1070/RC2011v080n03ABEH004162.

(76) Qi, P.; Chen, H.-L. L.; Nguyen, H. T. H.; Lin, C.-C. C.; Miller, S. A. Synthesis of Biorenewable and Water-Degradable Polylactam Esters from Itaconic Acid. Green Chem. 2016, 18 (15), 4170-4175. https://doi.org/10.1039/C6GC01081D.

(77) Mather, B. D.; Viswanathan, K.; Miller, K. M.; Long, T. E. Michael Addition Reactions in Macromolecular Design for Emerging Technologies. Prog. Polym. Sci. 2006, 31 (5), 487-531. https://doi.org/10.1016/j.progpolymsci.2006.03.001.

(78) Noordzij, G. J.; van den Boomen, Y. J. G.; Gilbert, C.; van Elk, D. J. P.; Roy, M.; Wilsens, C. H. R. M.; Rastogi, S. The Aza-Michael Reaction: Towards Semi-Crystalline Polymers from Renewable Itaconic Acid and Diamines. Polym. Chem. 2019, 4049-4058. https://doi.org/10.1039/c9py00463g.

(79) Noordzij, G.J; Roy, Manta; Bos, Natasja; Reinartz, V. W. Dicarboxylic Acid. Polymers (Basel). 2019, 11 (10), 1654-1668. https://doi.org/https://doi.org/10.3390/polym11101654.

(80) Paytash, P. L.; Sparrow, E.; Gathe, J. C. The Reaction of Itaconic Acid with Primary Amines. J. Am. Chem. Soc. 1950, 72 (3), 1415-1416. https://doi.org/10.1021/ja01159a520.

(81) Ai, X.; Wang, X.; Liu, J.; Ge, Z.; Cheng, T.; Li, R. An Effective Aza-Michael Addition of Aromatic Amines to Electron-de Fi Cient Alkenes in Alkaline Al 2 O 3. Tetrahedron 2010, 66 (29), 5373-5377. https://doi.org/10.1016/j.tet.2010.05.054.

(82) Okada, Y.; Banno, T.; Toshima, K.; Matsumura, S. Synthesis and Properties of Polycarboxylate-Type Green Surfactants with S- or N-Linkages. J. Oleo Sci. 2009, 58 (10), 519-528. https://doi.org/10.5650/jos.58.519.

(83) Kanitkar, A.; Aita, G.; Madsen, L. The Recovery of Polymerization Grade Aconitic Acid from Sugarcane Molasses. J. Chem. Technol. Biotechnol. 2013, 88 (12), 2188-2192. https://doi.org/10.1002/jctb.4084. 
(84) Nsengiyumva, O.; Miller, S. A. Synthesis, Characterization, and Water-Degradation of Biorenewable Polyesters Derived from Natural Camphoric Acid. 2019, 973-978. https://doi.org/10.1039/c8gc03990a.

(85) Malferrari, D.; Armenise, N.; Decesari, S.; Galletti, P.; Tagliavini, E. Surfactants from Itaconic Acid: Physicochemical Properties and Assessment of the Synthetic Strategies. ACS Sustain. Chem. Eng. 2015, 3 (7), 1579-1588. https://doi.org/10.1021/acssuschemeng.5b00264.

(86) Wang, Z.; Wei, T.; Xue, X.; He, M.; Xue, J.; Song, M.; Wu, S.; Kang, H.; Zhang, L.; Jia, Q. Synthesis of Fully Bio-Based Polyamides with Tunable Properties by Employing Itaconic Acid. Polym. (United Kingdom) 2014, 55 (19), 4846-4856.

https://doi.org/10.1016/j.polymer.2014.07.034.

(87) Pellis, A.; Hanson, P. A.; Comerford, J. W.; Clark, J. H.; Farmer, T. J. Enzymatic Synthesis of Unsaturated Polyesters: Functionalization and Reversibility of the Aza-Michael Addition of Pendants. Polym. Chem. 2019, 10 (7), 843-851. https://doi.org/10.1039/C8PY01655K.

(88) Wenker, $H$. The Synthesis of $\Delta 2$-Oxazolines and $\Delta 2$-Thiazolines from N-Acyl-2Aminoethanols. J. Am. Chem. Soc. 1935, 57 (6), 1079-1080.

https://doi.org/10.1021/ja01309a034.

(89) Fry, E. M. Oxazoline Ring-Opening. J. Org. Chem. 1950, 15 (4), 802-806. https://doi.org/10.1021/jo01150a014.

(90) Goldberg, A. A.; Kelly, W. 388. The Addition of Hydrogen Sulphide to 2-Phenyl- $\triangle 2$ Oxazoline and Benzoylethyleneimine: Attempted Synthesis of Fused ThiazolidineButyroazlactone Ring Systems. J. Chem. Soc. 1948, 1919-1926. https://doi.org/10.1039/JR9480001919.

(91) Baier, A.; Böhme, F.; Vogel, R.; Martin, H.; Leistner, D. Modification Reactions on Thermotropic Liquid-crystalline Poly (Ethylene Terephthalate-co-oxybenzoate) s with Bi-and Trivalent 2-oxazoline Couplers. Die Angew. Makromol. Chemie Appl. Macromol. Chem. Phys. 1995, 228 (1), 117-129. https://doi.org/10.1002/apmc.1995.052280109.

(92) Taylan, E.; Küsefoğlu, S. H. Chain Extension Reactions of Unsaturated Polyesters with Bis(2Oxazoline)S. J. Appl. Polym. Sci. 2012, 124 (4), 3229-3235. https://doi.org/10.1002/app.35450.

(93) Lustoň, J.; Kronek, J.; Kleinová, A.; Janigová, I.; Valentová, H.; Nedbal, J. Synthesis and Polymerization Reactions of Cyclic Imino Ethers. VI. Polymers with Biphenyl Structure. $J$. Polym. Sci. Part A Polym. Chem. 2012, 50 (19), 3936-3943. https://doi.org/10.1002/pola.26199.

(94) Inata, H.; Matsumura, S. Chain Extenders for Polyesters. VI. Properties of the Polyesters Chain-extended by 2,2'-bis(4H-3,1-benzoxazin-4-one). J. Appl. Polym. Sci. 1987, 34 (8), 2769-2776. https://doi.org/10.1002/app.1987.070340812.

(95) Huber, T.; Böhme, F.; Komber, H.; Kronek, J.; Luston, J.; Voigt, D.; Voit, B. New Hyperbranched Poly(Ether Amide)s via Nucleophilic Ring Opening of 2-OxazolineContaining Monomers. Macromol. Chem. Phys. 1999, 200 (1), 126-133. https://doi.org/10.1002/(SICI)1521-3935(19990101)200:1<126::AID-MACP126>3.0.CO;2-O.

(96) Cwik, A.; Hell, Z.; Heged??s, A.; Finta, Z.; Horv??th, Z. A Simple Synthesis of 2-Substituted 
Oxazolines and Oxazines. Tetrahedron Lett. 2002, 43 (22), 3985-3987.

https://doi.org/10.1016/S0040-4039(02)00723-2.

(97) Pirrung, M. C.; Tumey, L. N. Oxazoline Synthesis from Hydroxyamides by Resin Capture and Ring-Forming Release. J. Comb. Chem. 2000, 2 (6), 675-680.

https://doi.org/10.1021/cc000047g.

(98) Kangani, C. O.; Kelley, D. E.; Day, B. W. One Pot Direct Synthesis of Oxazolines, Benzoxazoles, and Oxadiazoles from Carboxylic Acids Using the Deoxo-Fluor Reagent. Tetrahedron Lett. 2006, 47 (37), 6497-6499. https://doi.org/10.1016/j.tetlet.2006.07.032.

(99) Kangani, C. O.; Day, B. W. A Novel and Direct Synthesis of 1,3,4-Oxadiazoles or Oxazolines from Carboxylic Acids Using Cyanuric Chloride/Indium. Tetrahedron Lett. 2009, 50 (38), 5332-5335. https://doi.org/10.1016/j.tetlet.2009.07.032.

(100) Wipf, P.; Venkatraman, S. From Aziridines to Oxazolines and Thiazolines: The Heterocyclic Route to Thiangazole. Synlett 2004, 1 (01), 1-10. https://doi.org/10.1055/s-1997-681.

(101) Zhou, P.; Blubaum, J. E.; Burns, C. T.; Natale, N. R. The Direct Synthesis of 2-Oxazolines from Carboxylic Esters Using Lanthanide Chloride as Catalyst. Tetrahedron Lett. 1997, 38 (40), 7019-7020. https://doi.org/10.1016/S0040-4039(97)01641-9.

(102) Phillips, A. J.; Uto, Y.; Wipf, P.; Reno, M. J.; Williams, D. R. Synthesis of Functionalized Oxazolines and Oxazoles with DAST and Deoxo-Fluor. Org. Lett. 2000, 2 (8), 1165-1168. https://doi.org/10.1021/o1005777b.

(103) Bigot, A.; Blythe, J.; Pandya, C.; Wagner, T.; Loiseleur, O. DAST-Mediated Cyclization of ??,??-Disubstituted-??- Acylaminoketones: Efficient and Divergent Synthesis of Unprecedented Heterocycles. Org. Lett. 2011, 13 (2), 192-195.

https://doi.org/10.1021/ol102454e.

(104) Bandgar, B. P.; Pandit, S. S. Direct Synthesis of 2-Oxazolines from Carboxylic Acids Using 2Chloro-4 , 6-Dimethoxy-1 , 3 , 5-Triazine under Mild Conditions. Tetrahedron Lett. 2003, 44 (11), 2331-2333. https://doi.org/10.1016/S0040-4039(03)00251-X.

(105) Karmee, S. K. Conversion of Fatty Acid Feedstocks to 2- Oxazoline. J. Oil Palm Res. 2015, 27 (3) (September), $261-265$.

(106) Tilvi and Keisham S. Singh, S. Synthesis of Oxazole, Oxazoline and Isoxazoline Derived Marine Natural Products: A Review. Curr. Org. Chem. 2016, 20 (8), 898-929. https://doi.org/10.2174/1385272819666150804000046.

(107) Frump, J. A. Oxazolines. Their Preparation, Reactions, and Applications. Chem. Rev. 1971, 71 (5), 483-505. https://doi.org/10.1021/cr60273a003.

(108) Wilsens, C. H. R. M.; Wullems, N. J. M.; Gubbels, E.; Yao, Y.; Rastogi, S.; Noordover, B. A. J. Synthesis, Kinetics, and Characterization of Bio-Based Thermosets Obtained through Polymerization of a 2,5-Furandicarboxylic Acid-Based Bis(2-Oxazoline) with Sebacic Acid. Polym. Chem. 2015, 6 (14), 2707-2716. https://doi.org/10.1039/c4py01609b.

(109) Xu, Q.; Li, Z. A Facile Synthesis of 2-Oxazolines Using a PPh3-DDQ System. Tetrahedron Lett. 2009, 50 (49), 6838-6840. https://doi.org/10.1016/j.tetlet.2009.09.127.

(110) Delidovich, I.; Hausoul, P. J. C.; Deng, L.; Pfützenreuter, R.; Rose, M.; Palkovits, R. 
Alternative Monomers Based on Lignocellulose and Their Use for Polymer Production. Chem. Rev. 2016, 116 (3), 1540-1599. https://doi.org/10.1021/acs.chemrev.5b00354.

(111) Becker, J.; Lange, A.; Fabarius, J.; Wittmann, C. Top Value Platform Chemicals: Bio-Based Production of Organic Acids. Curr. Opin. Biotechnol. 2015, 36 (Figure 1), 168-175. https://doi.org/10.1016/j.copbio.2015.08.022.

(112) Climent, M. J.; Corma, A.; Iborra, S. Conversion of Biomass Platform Molecules into Fuel Additives and Liquid Hydrocarbon Fuels. Green Chem. 2014, 16 (2), 516-547. https://doi.org/10.1039/c3gc41492b.

(113) Wilbon, P. A.; Chu, F.; Tang, C. Progress in Renewable Polymers from Natural Terpenes, Terpenoids, and Rosin. Macromol. Rapid Commun. 2013, 34 (1), 8-37. https://doi.org/10.1002/marc.201200513.

(114) Qi, P.; Chen, H. L.; Nguyen, H. T. H.; Lin, C. C.; Miller, S. A. Synthesis of Biorenewable and Water-Degradable Polylactam Esters from Itaconic Acid. Green Chem. 2016, 18 (15), 41704175. https://doi.org/10.1039/c6gc01081d.

(115) Li, P.; Ma, S.; Dai, J.; Liu, X.; Jiang, Y.; Wang, S.; Wei, J.; Chen, J.; Zhu, J. Itaconic Acid as a Green Alternative to Acrylic Acid for Producing a Soybean Oil-Based Thermoset: Synthesis and Properties. ACS Sustain. Chem. Eng. 2017, 5 (1), 1228-1236.

https://doi.org/10.1021/acssuschemeng.6b02654.

(116) Hoogenboom, R.; Schlaad, H. Bioinspired Poly(2-Oxazoline)S. Polymers (Basel). 2011, 3 (4), 467-488. https://doi.org/10.3390/polym3010467.

(117) Lava, K.; Verbraeken, B.; Hoogenboom, R. Poly(2-Oxazoline)s and Click Chemistry: A Versatile Toolbox toward Multi-Functional Polymers. Eur. Polym. J. 2015, 65, 98-111. https://doi.org/10.1016/j.eurpolymj.2015.01.014.

(118) Hoogenboom, R. Poly(2-Oxazoline)s: A Polymer Class with Numerous Potential Applications. Angew. Chemie - Int. Ed. 2009, 48 (43), 7978-7994. https://doi.org/10.1002/anie.200901607.

(119) Lava, K.; Verbraeken, B.; Hoogenboom, R. Poly(2-Oxazoline)s and Click Chemistry: A Versatile Toolbox toward Multi-Functional Polymers. Eur. Polym. J. 2015, 65, 98-111. https://doi.org/10.1016/j.eurpolymj.2015.01.014.

(120) Luxenhofer, R.; Han, Y.; Schulz, A.; Tong, J.; He, Z.; Kabanov, A. V; Jordan, R. Poly ( 2 Oxazoline ) s as Polymer Therapeutics. Macromol. Rapid Commun 2012, 33, 1613-1631. https://doi.org/10.1002/marc.201200354.

(121) Luxenhofer, R.; Huber, S.; Hytry, J.; Tong, J.; Kabanov, A. V.; Jordan, R. Chiral and WaterSoluble Poly(2-Oxazoline)S. J. Polym. Sci. Part A Polym. Chem. 2013, 51 (3), $732-738$. https://doi.org/10.1002/pola.26437.

(122) Cesana, S.; Auernheimer, J.; Jordan, R.; Kessler, H.; Nuyken, O. First Poly(2-Oxazoline)s with Pendant Amino Groups. Macromol. Chem. Phys. 2006, 207 (2), 183-192. https://doi.org/10.1002/macp.200500495.

(123) Nuyken, O.; Weberskirch, R.; Bortenschlager, M.; Schönfelder, D. Amphiphilic Polymers Based on Poly(2-Oxazoline)s - From ABC-Triblock Copolymers to Micellar Catalysis. Macromol. Symp. 2004, 215, 215-229. https://doi.org/10.1002/masy.200451118. 
(124) Cesana, S.; Kurek, A.; Baur, M. A.; Auernheimer, J.; Nuyken, O. Polymer-Bound Thiol Groups on Poly(2-Oxazoline)S. Macromol. Rapid Commun. 2007, 28 (5), 608-615. https://doi.org/10.1002/marc.200600737.

(125) Tauhardt, L.; Kempe, K.; Gottschaldt, M.; Schubert, U. S. Poly(2-Oxazoline) Functionalized Surfaces: From Modification to Application. Chem. Soc. Rev. 2013, 42 (20), 7998. https://doi.org/10.1039/c3cs60161g.

(126) Weber, C.; Hoogenboom, R.; Schubert, U. S. Temperature Responsive Bio-Compatible Polymers Based on Poly(Ethylene Oxide) and Poly(2-Oxazoline)S. Prog. Polym. Sci. 2012, 37 (5), 686-714. https://doi.org/10.1016/j.progpolymsci.2011.10.002.

(127) Hartlieb, M.; Kempe, K.; Schubert, U. S. Covalently Cross-Linked Poly(2-Oxazoline) Materials for Biomedical Applications-from Hydrogels to Self-Assembled and Templated Structures. J. Mater. Chem. B 2015, 3 (4), 526-538. https://doi.org/10.1039/c4tb01660b.

(128) Hoogenboom, R.; Fijten, M. W. M.; Thijs, H. M. L.; Van Lankvelt, B. M.; Schubert, U. S. Microwave-Assisted Synthesis and Properties of a Series of Poly(2-Alkyl-2-Oxazoline)S. Des. Monomers Polym. 2005, 8 (6), 659-671. https://doi.org/10.1163/156855505774597704.

(129) Böhme, F.; Clausnitzer, C.; Gruber, F.; Grutke, S.; Huber, T.; Pötschke, P.; Voit, B. Hyperbranched Poly(Ether Amide)s via Nucleophilic Ring Opening Reaction of Oxazolines. High Perform. Polym. 2001, 13 (2). https://doi.org/10.1088/0954-0083/13/2/303.

(130) Sano, Y. Polymerization of Bis(2-oxazoline) Compounds with Dicarboxylic Acids. J. Polym. Sci. Part A Polym. Chem. 1989, 27 (8), 2749-2760. https://doi.org/10.1002/pola.1989.080270820.

(131) Néry, L.; Lefebvre, H.; Fradet, A. Kinetic and Mechanistic Studies of Carboxylic AcidBisoxazoline Chain-Coupling Reactions. Macromol. Chem. Phys. 2003, 204 (14), 1755-1764. https://doi.org/10.1002/macp.200350036.

(132) Kempe, K. Chain and Step Growth Polymerizations of Cyclic Imino Ethers: From Poly(2Oxazoline)s to Poly(Ester Amide)S. Macromol. Chem. Phys. 2017, 201700021, 1-17. https://doi.org/10.1002/macp.201700021.

(133) Fazio, M. J. Nucleophilic Ring Opening of 2-Oxazolines with Amines: A Convenient Synthesis for Unsymmetrically Substituted Ethylenediamines. J. Org. Chem. 1984, 49 (25), 4889-4893. https://doi.org/10.1021/jo00199a029.

(134) Luston, J.; Böhme, F.; Komber, H.; Pompe, G. Unsaturated 2-Oxazoline Modification of Polyethylene Containing Carboxylic Groups. 2010, 1325. https://doi.org/10.1080/10601329808002099.

(135) Zhang, Y.; Broekhuis, A. A.; Picchioni, F. Thermally Self-Healing Polymeric Materials: The next Step to Recycling Thermoset Polymers? Macromolecules 2009, 42 (6), 1906-1912. https://doi.org/10.1021/ma8027672.

(136) Goiti, E.; Huglin, M. B.; Rego, J. M. Thermal Breakdown by the Retro Diels-Alder Reaction of Crosslinking in Poly[Styrene-Co-(Furfuryl Methacrylate)]. Macromol. Rapid Commun. 2003, 24 (11), 692-696. https://doi.org/10.1002/marc.200350013.

(137) Goussé, C.; Gandini, A.; Hodge, P. Application of the Diels-Alder Reaction to Polymers Bearing Furan Moieties. 2. Diels-Alder and Retro-Diels-Alder Reactions Involving Furan 
Rings in Some Styrene Copolymers. Macromolecules 1998, 31 (2), 314-321.

https://doi.org/10.1021/ma9710141.

(138) Sordo, F.; Mougnier, S. J.; Loureiro, N.; Tournilhac, F.; Michaud, V. Design of Self-Healing Supramolecular Rubbers with a Tunable Number of Chemical Cross-Links. Macromolecules 2015, 48 (13), 4394-4402. https://doi.org/10.1021/acs.macromol.5b00747.

(139) Chino, K.; Ashiura, M. Themoreversible Cross-Linking Rubber Using Supramolecular Hydrogen-Bonding Networks. Macromolecules 2001, 34 (26), 9201-9204. https://doi.org/10.1021/ma011253v.

(140) Betancourt, T.; Pardo, J.; Soo, K.; Peppas, N. A. Characterization of PH-Responsive Hydrogels of Poly(Itaconic Acid-g-Ethylene Glycol) Prepared by UV-Initiated Free Radical Polymerization as Biomaterials for Oral Delivery of Bioactive Agents. J. Biomed. Mater. Res. Part A 2010, 93 (1), 175-188. https://doi.org/10.1002/jbm.a.32510.

(141) Suckow, M.; Mordvinkin, A.; Roy, M.; Singha, N. K.; Heinrich, G.; Voit, B.; Saalwächter, K.; Böhme, F. Tuning the Properties and Self-Healing Behavior of Ionically Modified Poly(Isobutylene-Co-Isoprene) Rubber. Macromolecules 2018, 51 (2), 468-479. https://doi.org/10.1021/acs.macromol.7b02287.

(142) Barber, G. D.; Calhoun, B. H.; Moore, R. B. Poly(Ethylene Terephthalate) Ionomer Based Clay Nanocomposites Produced via Melt Extrusion. Polymer (Guildf). 2005, 46 (17), 67066714. https://doi.org/10.1016/j.polymer.2005.05.024.

(143) Leibler, L.; Rubinstein, M.; Colby, R. H. Dynamics of Reversible Networks. Macromolecules 1991, 24 (16), 4701-4707. https://doi.org/10.1021/ma00016a034.

(144) Cordier, P.; Tournilhac, F.; Soulié-Ziakovic, C.; Leibler, L. Self-Healing and Thermoreversible Rubber from Supramolecular Assembly. Nature 2008, 451 (7181), 977-980. https://doi.org/10.1038/nature06669.

(145) Stukalin, E. B.; Cai, L.-H. H.; Kumar, N. A.; Leibler, L.; Rubinstein, M. Self-Healing of Unentangled Polymer Networks with Reversible Bonds. Macromolecules 2013, 46 (18), 7525 7541. https://doi.org/10.1021/ma401111n.

(146) Tournilhac, F.; Cordier, P.; Montarnal, D.; Soulié-Ziakovic, C.; Leibler, L. Self-Healing Supramolecular Networks. Macromol. Symp. 2010, 291-292 (1), 84-88. https://doi.org/10.1002/masy.201050510.

(147) Imbernon, L.; Norvez, S.; Leibler, L. Stress Relaxation and Self-Adhesion of Rubbers with Exchangeable Links. Macromolecules 2016, 49 (6), 2172-2178. https://doi.org/10.1021/acs.macromol.5b02751.

(148) Denissen, W.; Droesbeke, M.; Nicola, R.; Leibler, L.; Winne, J. M.; Du Prez, F. E. Chemical Control of the Viscoelastic Properties of Vinylogous Urethane Vitrimers. Nat. Commun. 2017, 8. https://doi.org/10.1038/ncomms14857.

(149) Denissen, W.; De Baere, I.; Van Paepegem, W.; Leibler, L.; Winne, J.; Du Prez, F. E. Vinylogous Urea Vitrimers and Their Application in Fiber Reinforced Composites. Macromolecules 2018, 51 (5), 2054-2064. https://doi.org/10.1021/acs.macromol.7b02407.

(150) Guerre, M.; Taplan, C.; Nicolaÿ, R.; Winne, J. M.; Du Prez, F. E. Fluorinated Vitrimer Elastomers with a Dual Temperature Response. J. Am. Chem. Soc. 2018, 140 (41), 13272- 
13284. https://doi.org/10.1021/jacs.8b07094.

(151) Winne, J. M.; Leibler, L.; Prez, F. E. Du. Polymer Chemistry a Mechanistic Perspective. 2019, 6091-6108. https://doi.org/10.1039/c9py01260e.

(152) Stukenbroeker, T.; Wang, W.; Winne, J. M.; Du Prez, F. E.; Nicolaÿ, R.; Leibler, L. Polydimethylsiloxane Quenchable Vitrimers. Polym. Chem. 2017, 8 (43), 6590-6593. https://doi.org/10.1039/c7py01488k.

(153) Billiet, S.; Hillewaere, X. K. D.; Teixeira, R. F. A.; Prez, F. E. Du. Chemistry of Crosslinking Processes for Self-Healing Polymers. Macromol. Rapid Commun. 2013, No. 34, 290-309. https://doi.org/DOI: 10.1002/marc.201200689.

(154) Denissen, W.; Rivero, G.; Nicolaÿ, R.; Leibler, L.; Winne, J. M.; Prez, F. E. Du; Du Prez, F. E. Vinylogous Urethane Vitrimers. Adv. Funct. Mater. 2015, 25 (16), 2451-2457. https://doi.org/10.1002/adfm.201404553.

(155) Barner-kowollik, C.; Prez, F. E. Du; Espeel, P.; Hawker, C. J.; Junkers, T.; Schlaad, H.; Camp, W. Van. "Clicking” Polymers or Just Efficient Linking: What Is the Difference ?**. 2011, 60-62. https://doi.org/10.1002/anie.201003707.

(156) Kloxin, C. J.; Bowman, C. N. Covalent Adaptable Networks: Smart, Reconfigurable and Responsive Network Systems. Chem. Soc. Rev. 2013, 42 (17), 7161-7173. https://doi.org/10.1039/c3cs60046g.

(157) Podgórski, M.; Fairbanks, B. D.; Kirkpatrick, B. E.; McBride, M.; Martinez, A.; Dobson, A.; Bongiardina, N. J.; Bowman, C. N. Toward Stimuli-Responsive Dynamic Thermosets through Continuous Development and Improvements in Covalent Adaptable Networks (CANs). Adv. Mater. 2020, 1906876, 1-26. https://doi.org/10.1002/adma.201906876.

(158) Berg, G. J.; Gong, T.; Fenoli, C. R.; Bowman, C. N. A Dual-Cure, Solid-State Photoresist Combining a Thermoreversible Diels-Alder Network and a Chain Growth Acrylate Network. Macromolecules 2014, 47 (10), 3473-3482. https://doi.org/10.1021/ma500244r.

(159) Lyon, G. B.; Baranek, A.; Bowman, C. N. Scaffolded Thermally Remendable Hybrid Polymer Networks. Adv. Funct. Mater. 2016, 26 (9), 1477-1485.

https://doi.org/10.1002/adfm.201505368.

(160) Sowan, N.; Cox, L. M.; Shah, P. K.; Song, H. B.; Stansbury, J. W.; Bowman, C. N. Dynamic Covalent Chemistry at Interfaces: Development of Tougher, Healable Composites through Stress Relaxation at the Resin-Silica Nanoparticles Interface. Adv. Mater. Interfaces 2018, 5 (18), 1-10. https://doi.org/10.1002/admi.201800511.

(161) Hanzon, D. W.; Traugutt, N. A.; McBride, M. K.; Bowman, C. N.; Yakacki, C. M.; Yu, K. Adaptable Liquid Crystal Elastomers with Transesterification-Based Bond Exchange Reactions. Soft Matter 2018, 14 (6), 951-960. https://doi.org/10.1039/c7sm02110k.

(162) Lyon, G. B.; Cox, L. M.; Goodrich, J. T.; Baranek, A. D.; Ding, Y.; Bowman, C. N. Remoldable Thiol-Ene Vitrimers for Photopatterning and Nanoimprint Lithography. Macromolecules 2016, 49 (23), 8905-8913. https://doi.org/10.1021/acs.macromol.6b01281.

(163) Geng, H.; Wang, Y.; Yu, Q.; Gu, S.; Zhou, Y.; Xu, W.; Zhang, X.; Ye, D. Vanillin-Based Polyschiff Vitrimers: Reprocessability and Chemical Recyclability. ACS Sustain. Chem. Eng. 2018, 6 (11), 15463-15470. https://doi.org/10.1021/acssuschemeng.8b03925. 
(164) Azcune, I.; Odriozola, I. Aromatic Disulfide Crosslinks in Polymer Systems: Self-Healing, Reprocessability, Recyclability and More. Eur. Polym. J. 2016, 84, 147-160.

https://doi.org/10.1016/j.eurpolymj.2016.09.023.

(165) Fang, Z.; Zheng, N.; Zhao, Q.; Xie, T. Healable, Recon Fi Gurable, Reprocessable Thermoset Shape Memory Polymer with Highly Tunable Topological Rearrangement Kinetics. 2017. https://doi.org/10.1021/acsami.7b05713.

(166) Zhang, Y.; Ying, H.; Hart, K. R.; Wu, Y.; Hsu, A. J.; Coppola, A. M.; Kim, T. A.; Yang, K.; Sottos, N. R.; White, S. R.; et al. Malleable and Recyclable Poly(Urea-Urethane) Thermosets Bearing Hindered Urea Bonds. Adv. Mater. 2016, 28 (35), 7646-7651. https://doi.org/10.1002/adma.201601242.

(167) Zhou, Y.; Groote, R.; Goossens, J. G. P.; Sijbesma, R. P.; Heuts, J. P. A. Tuning PBT Vitrimer Properties by Controlling the Dynamics of the Adaptable Network. Polym. Chem. 2019, 10 (1), 136-144. https://doi.org/10.1039/c8py01156g.

(168) Al-Salem, S. M.; Lettieri, P.; Baeyens, J. Recycling and Recovery Routes of Plastic Solid Waste (PSW): A Review. Waste Manag. 2009, 29 (10), 2625-2643. https://doi.org/10.1016/j.wasman.2009.06.004.

(169) Pickering, S. J. Recycling Technologies for Thermoset Composite Materials-Current Status. Compos. Part A Appl. Sci. Manuf. 2006, 37 (8), 1206-1215. https://doi.org/10.1016/j.compositesa.2005.05.030.

(170) Kaneko, T.; Thi, T. H.; Shi, D. J.; Akashi, M. Environmentally Degradable, High-Performance Thermoplastics from Phenolic Phytomonomers. Nat. Mater. 2006, 5 (12), 966-970. https://doi.org/10.1038/nmat1778.

(171) Kint, D.; Muñoz-Guerra, S. A Review on the Potential Biodegradability of Poly(Ethylene Terephthalate). Polym. Int. 1999, 48 (5), 346-352. https://doi.org/10.1002/(SICI)10970126(199905)48:5<346::AID-PI156>3.0.CO;2-N.

(172) Noordzij, G. J. G. J.; Roy, M.; Bos, N.; Reinartz, V.; Wilsens, C. H. R. M. C. H. R. M. Improving the Hydrolysis Rate of the Renewable Poly(Hexamethylene Sebacate) Through Copolymerization of a Bis(Pyrrolidone)-Based Dicarboxylic Acid. Polymers (Basel). 2019, 11 (10), 1654. https://doi.org/10.3390/polym11101654.

(173) Paredes, N.; Rodríguez-Galán, A.; Puiggalí, J. Synthesis and Characterization of a Family of Biodegradable Poly(Ester Amide)s Derived from Glycine. J. Polym. Sci. Part A Polym. Chem. 1998, 36 (8), 1271-1282. https://doi.org/10.1002/(SICI)1099-0518(199806)36:8<1271::AIDPOLA10>3.0.CO;2-3.

(174) Li, J.; Xu, P. L.; Zhu, Y. K.; Ding, J. P.; Xue, L. X.; Wang, Y. Z. A Promising Strategy for Chemical Recycling of Carbon Fiber/Thermoset Composites: Self-Accelerating Decomposition in a Mild Oxidative System. Green Chem. 2012, 14 (12), 3260-3263. https://doi.org/10.1039/c2gc36294e.

(175) Boerman, M. A.; Van Der Laan, H. L.; Bender, J. C. M. E.; Hoogenboom, R.; Jansen, J. A.; Leeuwenburgh, S. C.; Van Hest, J. C. M. Synthesis of PH- and Thermoresponsive Poly(2-nPropyl-2-Oxazoline) Based Copolymers. J. Polym. Sci. Part A Polym. Chem. 2016, 54 (11), 1573-1582. https://doi.org/10.1002/pola.28011. 
(176) Sedlacek, O.; Monnery, B. D.; Filippov, S. K.; Hoogenboom, R.; Hruby, M. Poly(2Oxazoline)s - Are They More Advantageous for Biomedical Applications than Other Polymers? Macromol. Rapid Commun. 2012, 33 (19), 1648-1662. https://doi.org/10.1002/marc.201200453.

(177) Hoogenboom, R. Poly(2-Oxazoline)s: A Polymer Class with Numerous Potential Applications. Angew. Chemie - Int. Ed. 2009, 48 (43), 7978-7994. https://doi.org/10.1002/anie.200901607.

(178) Luxenhofer, R.; Schulz, A.; Roques, C.; Li, S.; Bronich, T. K.; Batrakova, E. V.; Jordan, R.; Kabanov, A. V. Doubly Amphiphilic Poly(2-Oxazoline)s as High-Capacity Delivery Systems for Hydrophobic Drugs. Biomaterials 2010, 31 (18), 4972-4979.

https://doi.org/10.1016/j.biomaterials.2010.02.057.

(179) de la Rosa, V. R. Poly(2-Oxazoline)s as Materials for Biomedical Applications. J. Mater. Sci. Mater. Med. 2014, 25 (5), 1211-1225. https://doi.org/10.1007/s10856-013-5034-y.

(180) Lustoň, J.; Kronek, J.; Böhme, F. Synthesis and Polymerization Reactions of Cyclic Imino Ethers. i. Ring-Opening Homopolyaddition of AB-Type Hydroxyphenyl-Substituted 2Oxazolines. J. Polym. Sci. Part A Polym. Chem. 2006, 44 (1), 343-355. https://doi.org/10.1002/pola.21159.

(181) Labrecque, L. V.; Kumar, R. A.; Davé, V.; Gross, R. A.; Mccarthy, S. P. Citrate Esters as Plasticizers for Poly(Lactic Acid). J. Appl. Polym. Sci. 1997, 66 (8), 1507-1513. https://doi.org/10.1002/(SICI)1097-4628(19971121)66:8<1507::AID-APP11>3.0.CO;2-0.

(182) Rodriguez-Galán, A.; Fuentes, L.; Puiggalı, J. Studies on the Degradability of a Poly(Ester Amide) Derived from L-Alanine, 1,12-Dodecanediol and 1,12-Dodecanedioic Acid. Polymer (Guildf). 2000, 41 (15), 5967-5970. https://doi.org/10.1016/S0032-3861(99)00756-9.

(183) Armelin, E.; Franco, L.; Rodríguez-Galán, A.; Puiggalí, J. Study on the Degradability of Poly(Ester Amide)s Related to Nylons and Polyesters 6,10 or 12,10. Macromol. Chem. Phys. 2002, 203 (1), 48-58. https://doi.org/10.1002/1521-3935(20020101)203:1<48::AIDMACP48>3.0.CO;2-E.

(184) Alla, A.; Rodríguez-Galán, A.; Muñoz-Guerra, S. Hydrolytic and Enzymatic Degradation of Copoly(Ester Amide)s Based on L-Tartaric and Succinic Acids. Polymer (Guildf). 2000, 41 (19), 6995-7002. https://doi.org/10.1016/S0032-3861(00)00070-7. 


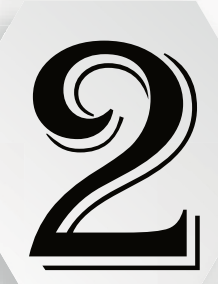

RENEWABLE (BIS)PYRROLIDONE MONOMERS AS COMPONENTS FOR THERMALLY CURABLE AND ENZYMATICALLY DEPOLYMERIZABLE 2-OXAZOLINE THERMOSET RESINS

Chapter partly based on:

Roy, M.; Noordzij, G. J.; Van Den Boomen, Y.; Rastogi, S.;

Wilsens, C. H. R. M., ACS Sustain. Chem. Eng. 2018, 6 (4), 5053-5066.

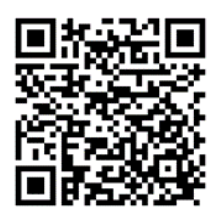




\title{
In this chapter...
}

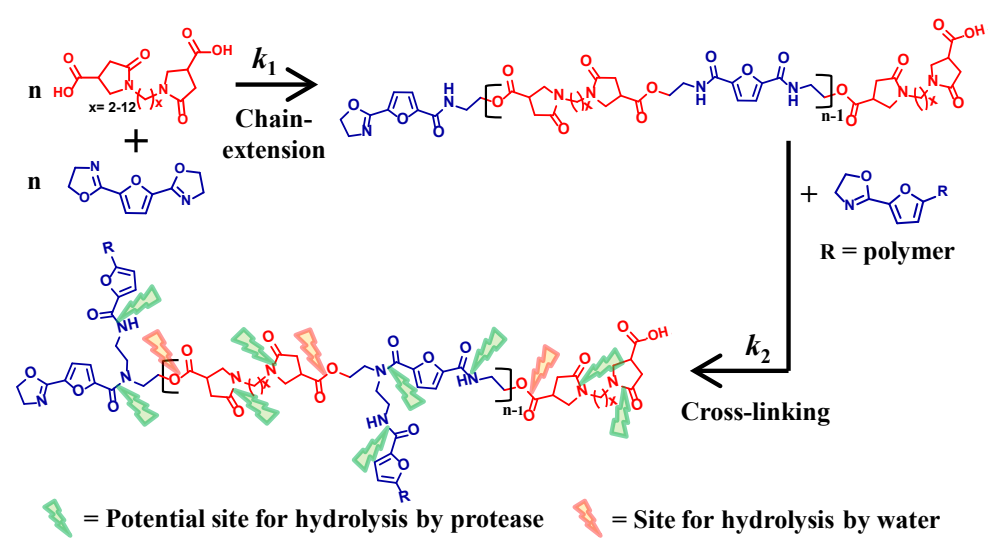

"Chain-extension $\left(k_{1}\right)$ and cross-linking $\left(k_{2}\right)$ reaction between renewable dicarboxylic acids and a 2,5-furandicarboxylic acid based bis(2-oxazoline) yielding fully renewable and enzymatically depolymerizable thermosets"

\begin{abstract}
This chapter describes the synthesis of (bis)pyrrolidone based dicarboxylic acids from itaconic acid via aza-Michael addition of a diamine using a catalytic amount of water and evaluates their potential application in 2-oxazoline resins for developing fully renewable thermoset materials. Later it is demonstrated that these (bis)pyrrolidone dicarboxylic acids exhibit significantly enhanced curing rates in 2oxazoline resins compared to sebacic acid. The rate of 2-oxazoline ring opening addition with carboxylic acid functionalities is determined by the used dicarboxylic acid, whereas the ring opening addition of the 2-oxazoline functionality with amide groups is determined by the used bis(2-oxazoline) compound. The thermosets obtained after curing proved to be readily plasticized by water, opening possibilities for enzymatic degradation.
\end{abstract}




\subsection{Introduction}

With the continuous growth of the plastic industry, an increasing amount of resources will be required to produce polymeric materials in the future. To this end, both academia and industry have a strong focus to develop renewable, recyclable, and/or degradable polymer materials that can ensure the sustainability of the polymer industry. Particular monomers that contribute to this endeavor are the renewable 2,5furandicarboxylic acid ${ }^{1,}$ and itaconic acid ${ }^{2,3}$. 2,5-furandicarboxylic acid, generally obtained from hydroxymethyl furfural or its derivatives ${ }^{4}$, has proven to exhibit excellent gas permeability properties in thermoplastic materials ${ }^{5}$ and has also proven applicable for thermosetting resins ${ }^{6}$. Itaconic acid, having two carboxylic functionalities combined with the presence of a vinyl group, has proven to be of interest for the synthesis of renewable polyesters $^{7-11}$, polyester resins ${ }^{12,13}$, polyamides ${ }^{14-17}$, and other polymers ${ }^{12,18-20}$. One particularly interesting application of itaconic acid is its ability to undergo the aza-Michael addition reaction with amines, followed by ring closure and the generation of a carboxylic acid functionalized pyrrolidone ring ${ }^{17,21}$. The resulting pyrrolidone based carboxylic acids are readily polymerized using conventional polycondensation methods, generally yielding amorphous and degradable polymer materials ${ }^{22-25}$. Especially the degradable nature of these carboxylic acids makes them interesting candidates for application in resins, as this opens possibilities for chemical recycling or full biodegradation of thermosets materials.

Though 2-oxazoline chemistry is often used to develop polymers for (bio)medical applications, as is reported by the groups of Hoogenboom ${ }^{26-29}$, Luxenhofer ${ }^{30-32}$, and Nuyken ${ }^{33,34}$, they are promising candidates for curing in resins for coatings or thermosets as they are highly reactive toward ring-opening addition with amines and carboxylic acid groups ${ }^{35-39}$. One particular challenge in the field of 2-oxazolines is related to their sustainability, as most widely used synthesis routes to obtain the 2-oxazoline moiety require nitriles ${ }^{40-42}$, haloalkylamides ${ }^{43}$, or aziridines ${ }^{44}$ as starting materials. That being said, an eco-friendly synthesis route involving the bulk amidation and consecutive ring formation of a carboxylic acid with an amino alcohol is known ${ }^{45,46}$, yielding the oxazolines functionality with only water as a reaction product.

Resins containing bis(2-oxazoline)s and dicarboxylic acids are known to undergo a thermal ring opening addition polymerization reaction, generating poly(ester-amide)s, which has been earlier communicated by groups of Bohme ${ }^{47-49}$, Sano $^{50,51}$, Nery ${ }^{52}$, and others ${ }^{37,39,53-56}\left(k_{1}\right.$ in Scheme 2.1). In turn, the generated amide groups are participating in a ring opening addition reaction with 2-oxazoline moieties, thereby forming tertiary amide bonds $\left(k_{2}\right.$ in Scheme 2.1). In fact, this mechanism is 
responsible for the cross-linking of the 2-oxazoline resins, where the cross-link density is controlled by the excess of bis(2-oxazoline) monomer ${ }^{50,51,57}$. In previous work we have demonstrated that the application of renewable monomers in 2oxazoline resins can be beneficial for the curing process: Besides being renewable in nature, the application of a 2,5-FDCA based bis(2-oxazoline) (FDCAox) showed to be selectively enhancing this cross-linking reaction, thereby significantly suppressing the required curing time.

In the present study we evaluate the performance of itaconic acid based (bis)pyrrolidone dicarboxylic acid monomers in 2-oxazoline resins (Scheme 2.1). The effect of the dicarboxylic acids on the curing process is explored using NMR spectroscopy, FTIR spectroscopy, and GPC chromatography. Additionally, the thermal behavior of the developed thermosets is investigated while particular attention is paid on the plasticizing effect of water in these materials. Lastly, preliminary enzymatic degradation studies are performed to evaluate whether these thermosets can be depolymerized in nature.

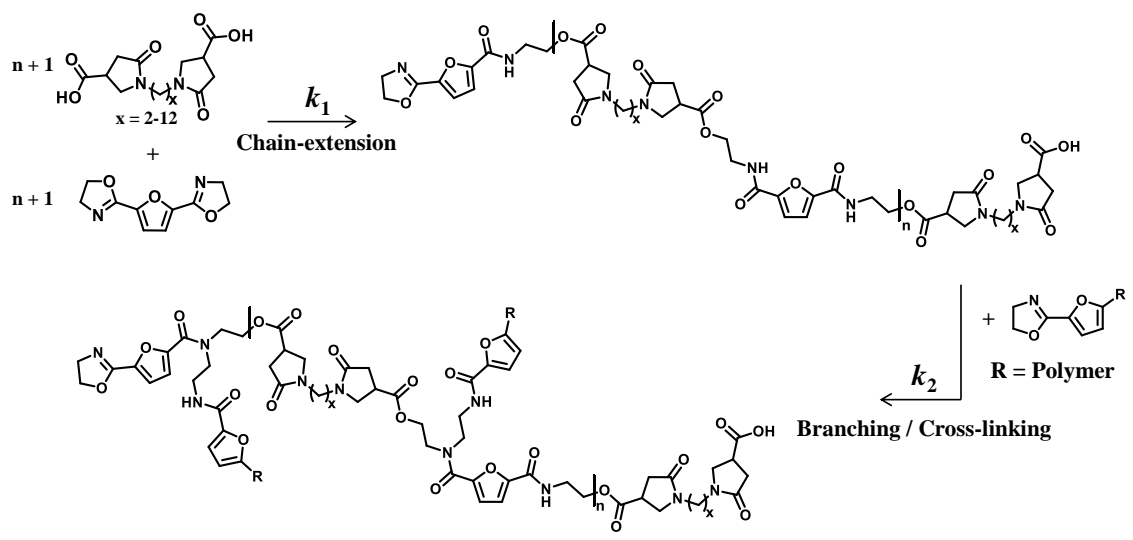

Scheme 2.1. Expected reactions occurring during thermally initiated ring-opening addition of FDCAox with the (bis)pyrrolidone dicarboxylic acid monomers explored in this study. 


\subsection{Experimental section}

\subsubsection{Materials}

Itaconic acid, 1,2-diaminoethane, 1,3-diaminopropane, 1,4-diaminobutane, 1,5-diaminopentane, 1,6-diaminohexane, 1,7-diaminoheptane, 1,8-diaminooctane, 1,9-diaminononane, 1,10-diaminodecane, and 1,12-diaminododecane were purchased from Sigma-Aldrich. Sebacic acid (SeA), 2-chloroethylamine hydrochloride, triphenylphosphite (TPP), thionyl chloride, sodium hydroxide, and potassium hydroxide were purchased from Sigma. 1,3-bis(4,5-dihydrooxazol-2yl)benzene (IAox) was purchased from TCI Europe. 2,5-furandicarboxylic acid (2,5FDCA) was obtained from Atomole, China (>99,5 wt\%, GC-MS). 2,5-bis(4,5dihydrooxazol-2-yl)furan (FDCAox) was synthesized according to previously reported procedure ${ }^{57}$. The chemicals are used as received, unless it is mentioned otherwise.

\subsubsection{General synthesis procedure of the (bis)pyrrolidone dicarboxylic acids}

The (bis)pyrrolidone based dicarboxylic acids were synthesized using a bulk reaction between itaconic acid and an $\alpha, \omega$-aliphatic diamine in a $2: 1$ molar ratio. The obtained monomers are abbreviated as $B P-C_{x}$ where $x$ denotes the methylene spacer length of the used diamine. As representative example, the synthesis of N,N'-octamethylene-bis(pyrrolidone-4-carboxylic acid), hence, BP-C 8 , is described: Itaconic acid $(9.02 \mathrm{~g}, 0.069 \mathrm{mmol})$ and 1,8-diaminooctane $(5 \mathrm{~g}, 0.035$ mmol) were added to a $100 \mathrm{ml}$ round bottom flask. The mixture was heated to 130 ${ }^{\circ} \mathrm{C}$ and was allowed to stir for 18 hours in the presence of a catalytic amount of distilled water to yield a yellow viscous liquid. The product was obtained as white crystals after recrystallization from a mixture methanol and ethyl acetate, followed by filtration and washing with ethyl acetate, and drying in vacuo overnight at $50{ }^{\circ} \mathrm{C}$. The yield of the synthesized monomer is shown in table 2.1. The yield is measured gravimetrically, while the purity is traced with ${ }^{1} \mathrm{H}$ NMR. ${ }^{1} \mathrm{H}$ NMR analysis is shown in figure $2.1\left(\mathrm{CDCl}_{3}+\mathrm{d}\right.$-TFA, $\left.300 \mathrm{MHz}\right): \delta 3.80(\mathrm{~m}, 4 \mathrm{H}), 3.44(\mathrm{~m}, 4 \mathrm{H}), 3.31(\mathrm{~m}, 2 \mathrm{H})$, $2.99(\mathrm{~d}, 2 \mathrm{H}, \mathrm{J}=7.9 \mathrm{~Hz}), 1.57(\mathrm{~m}, 4 \mathrm{H}), 1.30(\mathrm{~m}, 8 \mathrm{H}) .{ }^{13} \mathrm{C} \mathrm{NMR}\left(\mathrm{CDCl}_{3}+\mathrm{d}-\mathrm{TFA}, 300\right.$ $\mathrm{MHz}): \delta 178.2(C=\mathrm{OOH}), 175.0(\mathrm{NC}=\mathrm{O}), 49.7\left(\mathrm{NCH}_{2}\right.$ ring), $43.4\left(\mathrm{NCH}_{2}\right.$ spacer $)$, 35.7 ( $\mathrm{CH}$ ring), $33.8\left(\mathrm{CH}_{2}\right.$ ring), $28.6\left(\mathrm{CH}_{2}\right.$ spacer), $26.5\left(\mathrm{CH}_{2}\right.$ spacer $), 26.1\left(\mathrm{CH}_{2}\right.$ spacer). For the detailed overview of the synthesis and NMR analysis of the BP-C monomers, we refer to Appendix A (provided with QR code). 


\subsubsection{General melt polymerization procedure}

Reaction mixtures containing the desired molar ratio of $\mathrm{BP}-\mathrm{C}_{\mathrm{x}}$ and bis(2oxazoline) were mixed using a mortar and pestle. When desired, $1 \mathrm{wt} \%$ of triphenyl phosphite (TPP) was added as catalyst. The obtained reaction mixtures were polymerized on small scale (10 mg in a HPLC vial) or were polymerized on a 3-gram scale after loading into a $250 \mathrm{~mL}$ round bottom flask fitted with a mechanical stirrer. The polymerizations were performed at $180{ }^{\circ} \mathrm{C}$ for 60 minutes to ensure full conversion. Regular sampling was performed to monitor the reaction over time.

\subsubsection{Characterization methods}

${ }^{1} \mathrm{H}$ NMR and ${ }^{13} \mathrm{C}$ NMR spectroscopy was performed in a Bruker Ultrashield 300 spectrometer (300 MHz magnetic field). Samples were prepared by dissolving $10 \mathrm{mg}$ of monomer or polymer in $0.5 \mathrm{ml}$ deuterated dimethyl sulfoxide (DMSO- $d_{6}$ ), in a mixture of deuterated chloroform $\left(\mathrm{CDCl}_{3}\right)$ and deuterated trifluoroacetic acid (TFA- $d$ ), or in deuterated dimethylformamide (DMF- $\left.d_{7}\right)$. All spectra were referenced against tetramethyl silane (TMS).

Molecular weight of the synthesized poly(ester amide)s was determined via Gel Permeation Chromatography (GPC). The polymers $(5.0 \mathrm{mg})$ were dissolved in $1.5 \mathrm{ml}$ of 1,1,1,3,3,3-hexafluoroisopropanol (HFIP) containing 0,019\% sodium trifluoroacetate. After full dissolution, the mixtures were filtered over a $0.2 \mu \mathrm{m}$ PTFE syringe filter before injection. The GPC apparatus was calibrated with poly (methyl methacrylate) standards. Two PFG combination medium microcolumns with $7 \mu \mathrm{m}$ particle size (4.6 x $250 \mathrm{~mm}$, separation range 100-1.000.000 Da) and precolumn PFG combination medium with $7 \mu \mathrm{m}$ particle size $(4.6$ x $30 \mathrm{~mm})$ with Refractive Index detector (RI) were used in order to determine molecular weights and dispersities.

Attenuated total reflection Fourier transform infra-red spectroscopy (ATRFTIR) was performed using Perkin Elmer Spotlight 400 equipped with PIKE GladiATR, dual mode MCT (mercury cadmium telluride) detector with an array or a temperature-stabilized DTGS (deuterated triglycine sulfate) as a standard configuration. Polymerization and curing reactions were monitored on-line for one hour at the desired reaction temperature between $140{ }^{\circ} \mathrm{C}$ and $220^{\circ} \mathrm{C}$. Spectra were collected every two seconds in the range of 4000 to $450 \mathrm{~cm}^{-1}$ with a spectral resolution of $4 \mathrm{~cm}^{-1}$.

Thermal stability of the $B P-C_{x}$ monomers and poly(ester amide)s synthesized in this study was evaluated using thermogravimetric analysis (TGA). Experiments were performed on a TA Instruments TGA Q500 in a nitrogen rich atmosphere. Samples were heated from 20 to $700{ }^{\circ} \mathrm{C}$, at a heating rate of $10^{\circ} \mathrm{C} / \mathrm{min}$. 
Differential scanning calorimetry (DSC) was performed to identify the thermal transitions of the developed materials using a TA Instruments DSC Q2000. Two heating and cooling runs were performed at heating and cooling rates of $10{ }^{\circ} \mathrm{C} / \mathrm{min}$. The melting temperature $\left(T_{\mathrm{m}}\right)$ was determined from the first heating run while the glass transition temperatures of the $\mathrm{BP}-\mathrm{C}_{\mathrm{x}}$ monomers were determined from the second heating run.

\subsubsection{Enzymatic depolymerization and solubilization procedures}

The solubility and enzymatic degradation were evaluated on the developed thermosets obtained after curing of an equimolar mixture bis(2-oxazoline) and BP$\mathrm{C}_{\mathrm{x}}$ monomers for 1 hour at $180{ }^{\circ} \mathrm{C}$. In general, $30 \mathrm{mg}$ of the thermoset was added to water or tris buffer solution $(1 \mathrm{~mL}, 100 \mathrm{mM}$, pH8), with or without Bascillus $S p$ (0.8 $\mathrm{ml}, 16$ units/mL, Sigma Aldrich) enzyme. The enzyme was added to the polymer together with a $\mathrm{CaCl}_{2}$ solution $(0.01 \mathrm{M})$ at $50{ }^{\circ} \mathrm{C}$ in order to enforce depolymerization and dissolve the poly(ester amide) thermosets ${ }^{58-60}$.

\subsection{Results and discussion}

\subsection{1 (Bis)pyrrolidone based dicarboxylic acid $\left(B P-C_{x}\right)$ synthesis}

Previous reports on (bis)pyrrolidone based dicarboxylic acids from itaconic acid from different aliphatic diamine spacers (Scheme 2.2) often involve synthesis from water as both reaction medium and catalyst ${ }^{11,17}$. These dicarboxylic acids, generally obtained as viscous oily liquids, can be used directly for polymerization or are first isolated after several trituration steps ${ }^{15}$. One potential problem with these methods is that residual unreacted primary or secondary amine groups may be present in the final product. The presence of such amine impurities is detrimental for thermal curing polymerizations with 2-oxazolines as they are known to negatively affect the reactivity of the mixture ${ }^{61}$. Additionally, the presence of unreacted itaconic acid or amines will affect the stoichiometry and might hamper build-up of molecular weight. For this purpose, we have slightly modified the synthesis and purification methods provided in literature ${ }^{11,15}$ : Firstly the synthesis is performed in bulk at $130-180{ }^{\circ} \mathrm{C}$ in the presence of a catalytic amount of water. After reaction overnight, the formed oily yellow liquid is subjected to reduced pressure to remove the water generated during the reaction. Secondly, the monomers are dissolved and recrystallized from a methanol / ethyl acetate mixture to obtain them in high purity. 


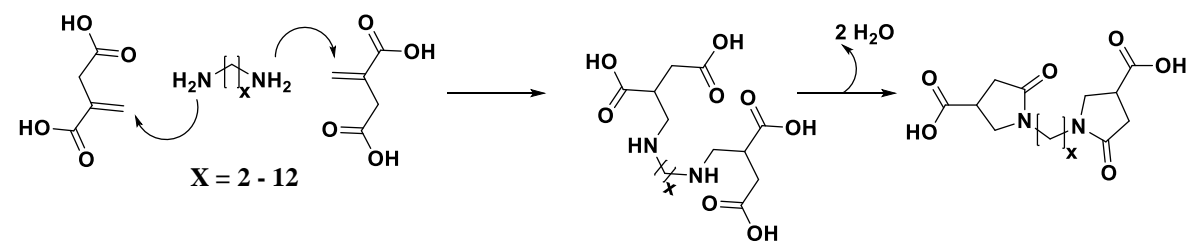

Scheme 2.2. Aza-Michael addition of various diamines with itaconic acid, followed by intramolecular cyclization. The ensuing monomers are abbreviated as $B P-C_{x}$, where $x$ stands for the number of methylene groups in the used diamine spacer. For example, the N,N'-octamethylene-bis(pyrrolidone-4-carboxylic acid) monomer based on 1,8-diaminooctane is named $B P-C_{8}$.

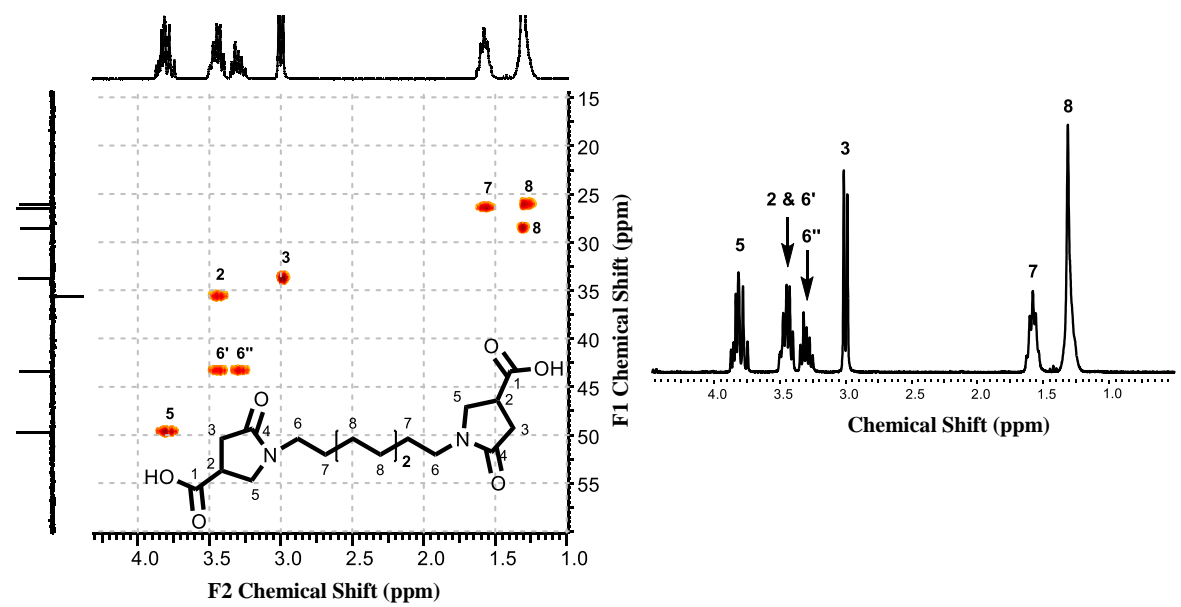

Figure 2.1. HSQC $\left({ }^{1} \mathrm{H}_{-}{ }^{13} \mathrm{C} \mathrm{DEPT)}\right.$ spectrum (left) and ${ }^{l} \mathrm{H}-\mathrm{NMR}$ spectrum (right) and the corresponding peak assignment of the BP-C $C_{8}$ monomer, taken in $C D C l_{3} / d-T F A$ as solvent.

With respect to the yield of the monomers after purification, we observe that the recrystallization process proceeds rapidly and results in high isolated yields for monomers with short $\left(\mathrm{BP}-\mathrm{C}_{2}, \mathrm{BP}-\mathrm{C}_{3}\right.$, and $\left.\mathrm{BP}-\mathrm{C}_{4}\right)$ or long $\left(\mathrm{BP}-\mathrm{C}_{10}\right.$ and $\left.\mathrm{BP}-\mathrm{C}_{12}\right)$ diamine spacers. However, recrystallization proved more challenging for the $\mathrm{BP}-\mathrm{C}_{\mathrm{x}}$ monomers having 5 to 9 methylene spacers between the pyrrolidone rings. Especially when using odd diamine spacers, recrystallization from solvent proved challenging, resulting in decreased isolated yields as is visible from Table 2.1. In fact, the BP-C 7 monomer proved impossible to crystallize using the described purification procedure, even upon prolonged recrystallization times at $-20{ }^{\circ} \mathrm{C}$. 
Table 2.1. Yield, thermal stability and thermal behavior of the BP-C $C_{x}$ monomers.

\begin{tabular}{|c|c|c|c|c|}
\hline Entry & Yield $(\%)^{a}$ & $T_{\mathrm{m}}\left({ }^{\circ} \mathbf{C}\right)^{\mathbf{b}}$ & $T_{\mathrm{g}}\left({ }^{\circ} \mathrm{C}\right)^{\mathrm{b}}$ & $T_{\mathrm{d}}\left({ }^{\circ} \mathbf{C}\right)^{\mathrm{c}}$ \\
\hline $\mathrm{BP}-\mathrm{C}_{2}$ & 95 & 238 & 48.5 & 272 \\
\hline BP-C 3 & 90 & 194 & 31.3 & 269 \\
\hline BP-C 4 & 85 & 196 & 24.1 & 274 \\
\hline BP-C 5 & 65 & 156 & 22.3 & 275 \\
\hline $\mathrm{BP}_{-\mathrm{C}_{6}}$ & 75 & 181 & 18.7 & 272 \\
\hline BP-C8 & 85 & 153 & 12.6 & 274 \\
\hline BP-C9 & 30 & 81 & 4.9 & 271 \\
\hline BP-C 10 & 90 & 131 & 3.1 & 274 \\
\hline BP-C 12 & 90 & 145 & 3.7 & 271 \\
\hline
\end{tabular}

a'Yield determined after purification by recrystallization. ${ }^{b}$ Determined from the DSC analysis, whereas the peak melting temperature $\left(T_{\mathrm{m}}\right)$ is determined from the first heating run and the glass transition temperature $\left(T_{\mathrm{g}}\right)$ is determined from the second heating run. ${ }^{\mathrm{c}}$ Thermal degradation temperature determined from TGA analysis, where the $T_{\mathrm{d}}$ denotes the onset point for degradation.

The obtained BP-C $\mathrm{C}_{\mathrm{x}}$ crystallites were tested for their thermal stability and melting behavior using TGA and DSC analysis. Overall, the monomers are stable up to temperatures well above their melting temperatures (Figure 2.2 and Table 2.1): In general, an onset of degradation is observed around $270{ }^{\circ} \mathrm{C}$ for all monomers, and no significant weight loss is detected below $250{ }^{\circ} \mathrm{C}$. With respect to the thermal transitions prior to degradation, the $\mathrm{BP}-\mathrm{C}_{\mathrm{x}}$ monomers display distinct melting behavior $\left(T_{\mathrm{m}}\right)$ during the first heating run in DSC analysis. During the second DSC heating run cold-crystallization and consecutive melting is observed only for the BP- $\mathrm{C}_{2}, \mathrm{BP}-\mathrm{C}_{8}$ and BP- $\mathrm{C}_{12}$ monomers, as is displayed in Figure 2.3A for BP-C $\mathrm{C}_{8}$ All other monomers do not crystallize within the time provided by the DSC analysis methods, but instead display a characteristic glass transition temperature $\left(T_{\mathrm{g}}\right)$. The presence of such a $T_{\mathrm{g}}$ likely originates from the hydrogen bonding between dicarboxylic acid groups, resulting in long range order. Indeed, this seems to be the case as the $T_{\mathrm{g}}$ of the BP-C $\mathrm{C}_{\mathrm{x}}$ monomers (Figure 2.3B) decreases rather linearly with 
the hydrogen bonding density of the carboxylic acid groups, as is explained in Appendix A (Figures A3\&A4).

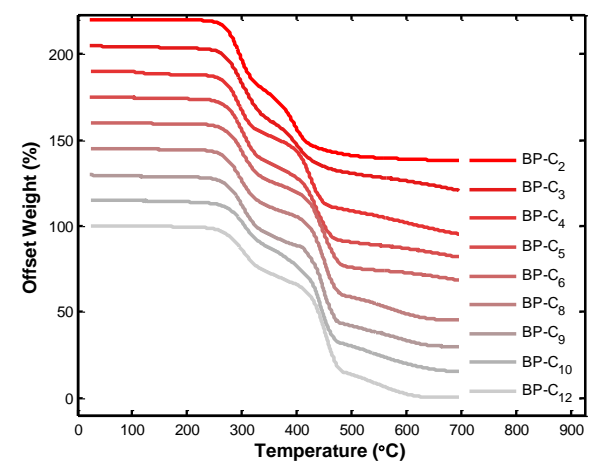

Figure 2.2. Offset TGA thermograms depicting the thermal stability of the various $B P-C_{x}$ monomers developed in this study. The applied heating rate for the TGA experiments was $10^{\circ} \mathrm{C} / \mathrm{min}$.
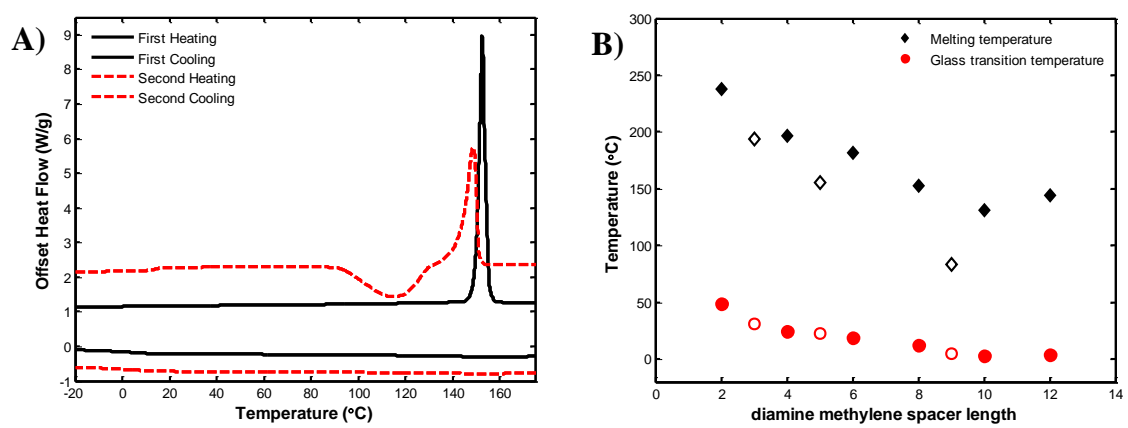

Figure 2.3. A) DSC thermogram of $B P-C_{8}$ depicting the characteristic melting behavior of purified BP-C $C_{x}$ monomers. B) Overview of the peak melting temperatures of the synthesized monomers observed during the first DSC heating run, and the glass transition temperature observed during the second DSC heating run. All heating and cooling rates were $10^{\circ} \mathrm{C} / \mathrm{min}$. Note, the filled symbols depict the thermal transitions of the BP-C $C_{x}$ monomers containing even amine spacers, whereas the open symbols depict the thermal transitions of the BP-C $C_{x}$ monomers with odd amine spacers.

An overview of all the peak melting temperatures observed during the first heating run and the glass transition temperatures observed during the second heating run is depicted in Figure 2.3B. In general, the melting temperature of the $B P-C_{x}$ monomers decreases with increasing methylene spacer length. For example, the BP- 
$\mathrm{C}_{2}$ monomer exhibits a peak melting temperature at $238{ }^{\circ} \mathrm{C}$, whereas the $\mathrm{BP}-\mathrm{C}_{12}$ monomer melts already at $145^{\circ} \mathrm{C}$. Additionally, a characteristic odd-even effect ${ }^{62,63}$ is observed: The $B P-C_{x}$ monomers with an odd amine spacer are displaying significantly lowered melting temperatures than the $B P-C_{x}$ monomers containing even diamine spacers. This low melting temperature of $\mathrm{BP}-\mathrm{C}_{\mathrm{x}}$ monomers with odd amine spacers explains their previously observed low isolated yield: $B P-C_{x}$ monomers with odd amine spacers can only form defected crystallites with low melting temperatures during recrystallization from solvent. As a consequence, crystal growth is significantly hindered, thereby lowering the isolated yield after recrystallization.

Overall, based on the synthesis procedure and the behavior of the synthesized $\mathrm{BP}-\mathrm{C}_{\mathrm{x}}$ monomers we can conclude that the modified bulk synthesis allows for upscaling in good yield when using diamine spacers that generate high melting $\mathrm{BP}-\mathrm{C}_{\mathrm{x}}$ monomers.

\subsubsection{Curing performance of BP-C $C_{x}$ monomer in 2-oxazoline resins}

The BP- $\mathrm{C}_{\mathrm{x}}$ monomers were used as dicarboxylic acid for the preparation of 2-oxazoline resins. To avoid the use of solvents, the monomers were grinded in the solid state and polymerized in bulk. In this study, the evaluated bis(2-oxazoline) monomers are 1,3-bis(4,5-dihydrooxazol- 2-yl)benzene (IAox) and the renewable 2,5-bis(4,5-dihydrooxazol-2-yl)furan (FDCAox). An overview of the used monomers for polymerization reactions is provided in Scheme 2.3. The characteristic polymerization reaction of $B P-C_{x}$ monomers with FDCAox is depicted in Scheme 2.1. In general, the ring-opening polyaddition reaction between dicarboxylic acids and bis(2-oxazoline)s $\left(k_{1}\right)$ yields linear poly(ester-amide)s $\mathrm{s}^{57}$. However, the formed amide bond is susceptible to react with another 2-oxazoline moiety $\left(k_{2}\right)$, thereby providing the means to generate branched or cross-linked structures. In previous work we have demonstrated that the branching and cross-linking reaction is highly dependent on the selected bis(2-oxazoline spacer): To recall, the amorphous 2,5furandicarboxylic acid based amide groups are highly susceptible to participate in a branching reaction with a 2-oxazoline ${ }^{57}$. 
<smiles>c1cc(C2=NCCO2)oc1C1=NCCO1</smiles>

FDCAox

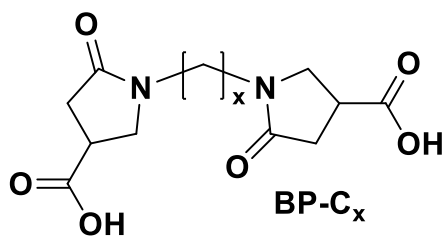<smiles>c1cc(C2=NCCO2)cc(C2=NCCO2)c1</smiles>

IAox<smiles>CC(CCCC(=O)O)C(=O)O</smiles>

Sebacic acid

Scheme 2.3. Overview of the used monomers for polymerization reactions performed in this study.

The curing performance of the $\mathrm{BP}-\mathrm{C}_{\mathrm{x}}$ monomers in 2-oxazoline resins was tested in equimolar systems containing either FDCAox or IAox. As reference, the reaction with sebacic acid as aliphatic dicarboxylic acid was performed. The equimolar systems were cured for one hour at $180^{\circ} \mathrm{C}$ under nitrogen atmosphere. The conversion and molecular weights were determined through ${ }^{1} \mathrm{H}$ NMR and GPC analysis of samples taken at regular time intervals. The conversion determination procedure from NMR analysis and representative NMR spectra are provided in Appendix A (provided with QR code). Figure 2.4 depicts the conversion over time for the first 5 minutes of reaction time for the tested reaction systems: In general, we observe that all systems go to roughly $90 \%$ conversion within this reaction time. Such a high reactivity is expected as the reaction of 2-oxazolines with dicarboxylic acids is known to proceed sufficiently fast at this reaction temperature to allow for chainextension reactions in compounding or extrusion processes. Interestingly, we observe that resins with $\mathrm{BP}_{-} \mathrm{C}_{8}$ react significantly faster compared to systems containing sebacic acid as they already achieve more than 50\% conversion within 20 seconds of reaction time. Additionally, as reported in previous work, resins containing 2,5FDCAox exhibit a higher reactivity than systems containing IAox (Figure 2.4), which is attributed to the increased tendency of FDCAox to undergo cross-linking with the formed amide groups ${ }^{57}$. 


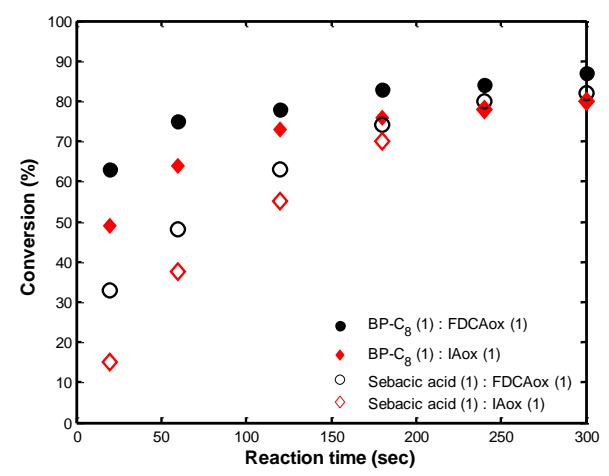

Figure 2.4. Conversion calculated from NMR analysis during the first 5 minutes of curing of the various 2-oxazoline resins at $180^{\circ} \mathrm{C}$. Characteristic NMR spectra and calculation method are provided in Appendix A (provided with QR code).

To obtain more information on the molecular weight build-up at the start of the curing process, systems containing various $B$ P- $C_{x}$ monomers and IAox have been cured for 1 minute at $180{ }^{\circ} \mathrm{C}$ and their molecular weights were evaluated through HFIP-GPC analysis. Please note that we confine ourselves to IAox based systems for the determination of molecular weights given the high tendency of FDCAox based resins to undergo rapid cross-linking, thereby limiting their solubility. As is visible from Figure 2.5, the molecular weights $\left(M_{\mathrm{w}}\right)$ of the systems containing the BP-C $\mathrm{x}_{\mathrm{x}}$ monomers reach $10-20 \mathrm{~kg} / \mathrm{mol}$ within only 1 minute of curing.
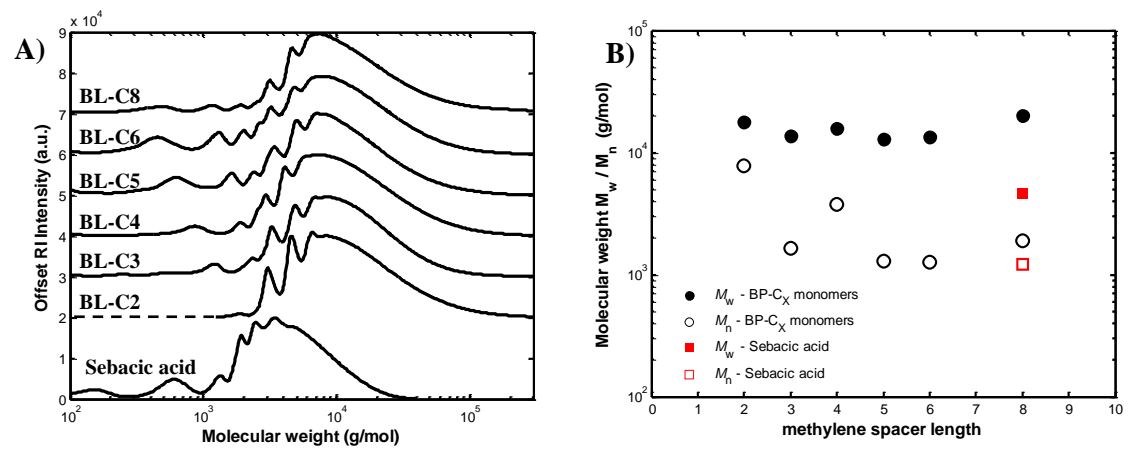

Figure 2.5. A) GPC traces of equimolar systems containing dicarboxylic acid and IAox cured for 1 minute at $180{ }^{\circ} \mathrm{C}$. B) Overview of corresponding molecular weights $\left(M_{w}\right.$ and $\left.M_{n}\right)$ of the GPC traces shown in Figure 2.5A.

In contrast, the system containing sebacic acid only yields a $M_{\mathrm{w}}$ of 4.5 $\mathrm{kg} / \mathrm{mol}$ after the same reaction time. Additionally, the presence of monomers and 
oligomers can be detected in the GPC traces of all systems, confirming that no full conversion is yet achieved (Figure 2.5A). Furthermore, in all BP-C $\mathrm{C}_{\mathrm{x}}$ GPC traces a high molecular weight tail is observed, likely indicating the presence of branched structures.

To obtain more information on the polymerization proceeding over time, the IAox based resins containing sebacic acid and $\mathrm{BP}-\mathrm{C}_{8}$ were cured for one hour and their molecular weight build-up was monitored over time using GPC analysis. As is shown in the GPC traces of the IAox: $\mathrm{BP}-\mathrm{C}_{8}$ resin (Figure 2.6A), a rapid molecular weight build-up is achieved immediately upon melting. Over time, the residual monomers and oligomers continue to react and the high molecular weight tail increases, but overall, no significant changes are observed in the GPC traces. This experiment confirms that systems with $\mathrm{BP}-\mathrm{C}_{8}$ exhibit extremely high reactivity and rapidly build up molecular weight. Furthermore, after 60 minutes of curing, the sample proved to be only partially insoluble in the HFIP solvent, indicating that branching and cross-linking proceeds over time.
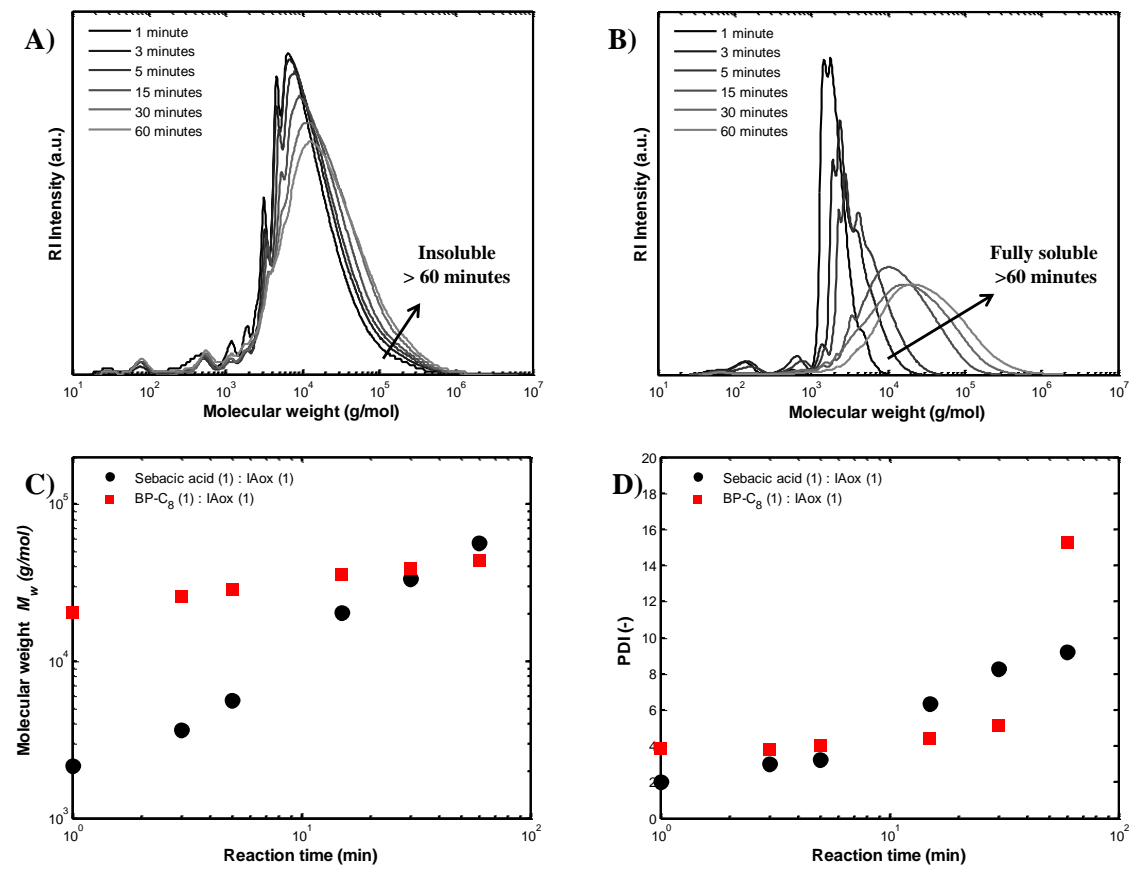

Figure 2.6. Molecular weight build-up according to GPC analysis during curing at

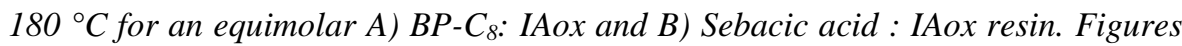
$C)$ and $D)$ depict the increase in $M_{w}$ and PDI over time respectively for both systems. 
In contrast, when looking at the GPC traces of the sebacic acid-based resin (Figure 2.6B) we observe a more gradual increase in molecular weight over time. Such behavior is characteristic for polycondensation type of polymerizations. Additionally, when the polymerization reaches high conversions, a rise in high molecular weight tail and $M_{\mathrm{w}}$ is observed together with a systematic increase in dispersity, indicating that branching also occurs in this system (Figures 2.6C and 2.6D). That being said, branching and cross-linking proceeds significantly slower in the presence of sebacic acid compared to samples containing BP- $\mathrm{C}_{8}$ as the system remains fully soluble in HFIP, even after 6 hours of reaction time.

From the previous observations we have strong indications that the BP- $C_{x}$ monomers significantly enhance the reaction rate of 2-oxazoline resins compared to aliphatic dicarboxylic acids. However, at this point it is not clear whether the presence of $\mathrm{BP}-\mathrm{C}_{\mathrm{x}}$ selectively accelerates the $k_{1}$ (chain extension) or $k_{2}$ (branching) reaction constant as both processes seem to proceed simultaneously. In order to gain more information on this reactivity difference, on-line monitoring of the polymerization is performed using ATR-FTIR analysis. For these reactions a carboxylic acid to (2oxazoline) ratio of $1: 2$ has been used to enforce cross-linking and to assess the $k_{2}$ reaction constant: The excess of 2-oxazoline moieties ensure a rapid conversion of the dicarboxylic acids into ester-amide bonds in the initial stage of polymerization, leaving the residual 2-oxazoline moieties only with the amide groups to react. The reaction of the 2-oxazoline moiety with the secondary amide yields a tertiary amide bond (Scheme 2.1), which exhibits a characteristic resonance in FTIR spectroscopy around $1417 \mathrm{~cm}^{-1}$. Figures $2.7 \mathrm{~A}$ and $2.7 \mathrm{~B}$ depict the characteristic FTIR spectra obtained during a polymerization of FDCAox based resins containing BP-C $\mathrm{C}_{8}$ and sebacic acid as dicarboxylic acid, respectively.

The FTIR spectra obtained during polymerization of systems containing IAox as bis(2-oxazoline) are provided in Appendix A (Figure A3). Three distinct vibrational bands are highlighted in Figure 2.7: I correspond to the furan ring vibration $\left(816 \mathrm{~cm}^{-1}\right)$, II corresponds to the 2-oxazoline ring vibration $\left(922 \mathrm{~cm}^{-1}\right)$, and III corresponds to the tertiary amide bonds vibration $\left(1412 \mathrm{~cm}^{-1}\right)$. In general, for both systems we observe that peak area $\mathbf{I}$ remains constant during the curing process, indicating that no evaporation of the FDCAox occurs over time. The fact that no components are evaporating over time allow us to use this vibration as internal standard. Signals II and III change over time as a result of the proceeding chemical reaction, as the 2-oxazoline ring is depleted during ring-opening (signal II) and tertiary amide bonds are formed upon cross-linking (signal III).

When qualitatively comparing the change in tertiary amide bond signal (III) over time for the four tested systems, we can identify two characteristic regimes in 
the polymerization (Figure 2.8). Regime 1 denotes the region where mostly melting of the mixture proceeds in combination with reaction of the 2-oxazoline moieties with carboxylic acid groups until a plateau value is observed. According to the NMR analysis depicted in Figure 2.4 this generally proceeds within the first minutes of polymerization. Regime 2 denotes the region where the remaining 2-oxazolines react with the generated amide bonds, hence the cross-linking of the system is proceeding. Obviously, these regimes differ per reaction and depend on temperature, reaction rate and the presence of a catalyst. For this reason, non-indicative lines separating the two regimes are added to guide the eye.
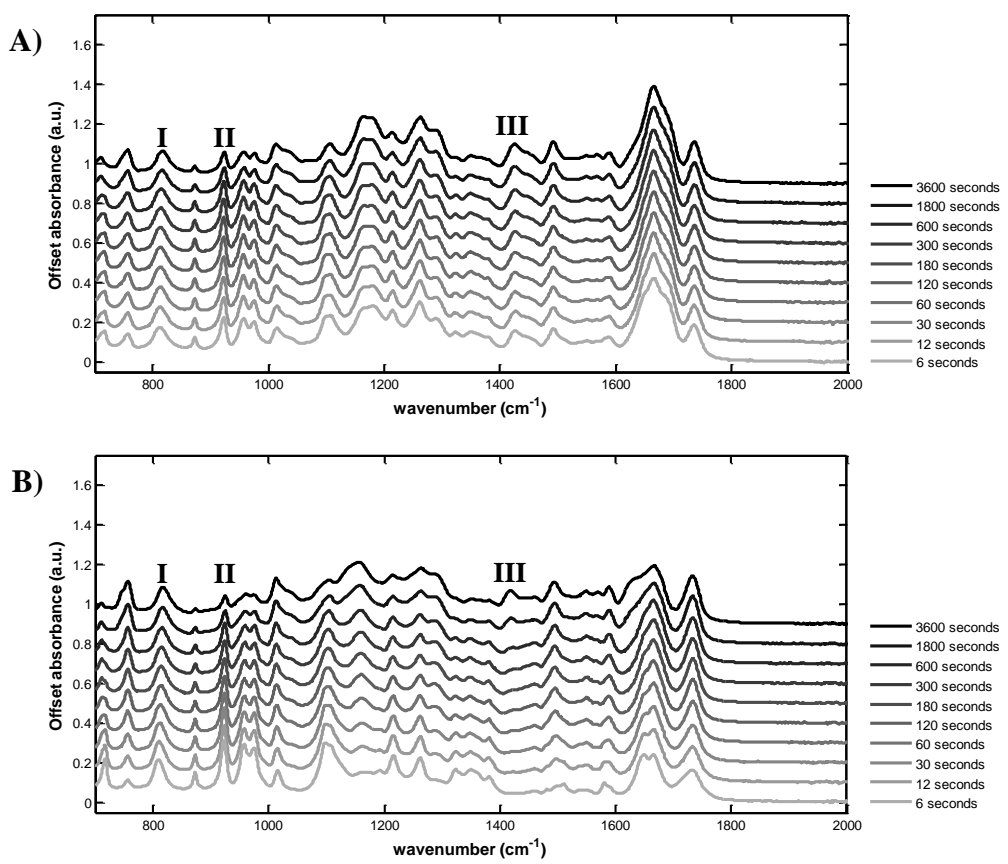

Figure 2.7. FTIR spectra $\left(700-2000 \mathrm{~cm}^{-1}\right)$ obtained during the on-line monitoring of the curing of (A) BP-C $:$ FDCAox and (B) Sebacic acid: FDCAox systems at 180 ${ }^{\circ} C$. Both polymerizations were performed in a dicarboxylic acid : 2-oxazoline ratio of 1 : 2. Vibrational bands I, II, and III denote the characteristic vibrations from the furan ring, 2-oxazoline ring, and tertiary amide bonds respectively.

Interestingly, systems containing $\mathrm{BP}-\mathrm{C}_{8}$ tend to melt rapidly and reach a plateau value within seconds, as is visible from Figure 2.8A. However, the crosslinking of the reaction mixtures seems to depend mostly on the selected bis(2oxazoline) spacer, as only the systems containing FDCAox tend to rapidly crosslink 
over time. Systems containing IAox do exhibit cross-linking, but at a significantly decreased rate compared to FDCAox based systems. The addition of triphenyl phosphite (TPP) as catalyst, does facilitate the cross-linking reaction for all systems (Figure 2.8B), indicating that $k_{2}$ reaction constant is determined by either the selected bis(2-oxazoline) or catalyst, but not by the dicarboxylic acid. The FTIR spectra obtained during polymerization of systems containing $1 \mathrm{wt} \%$ TPP used for the generation of Figure 2.8B are provided in Appendix A (Figure A4).
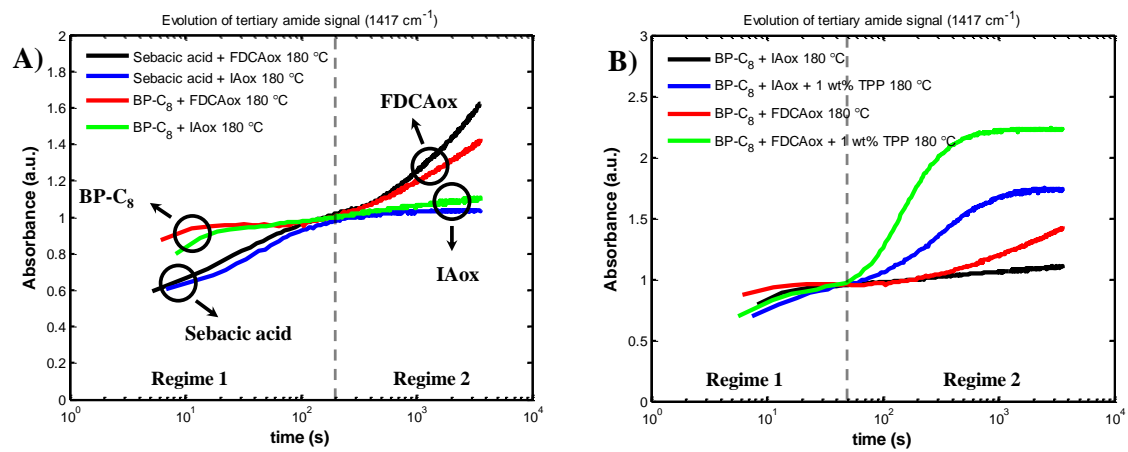

Figure 2.8. Changes of the normalized tertiary amide vibration III during polymerization of dicarboxylic acid: bis(2-oxazoline) resins (ratio of $1: 2$ ) at $180^{\circ} \mathrm{C}$. The curves are freely shifted vertically to align the plateau values obtained at the end of regime 1 to ease the comparison.

To obtain quantitative insight on the effect of $\mathrm{BP}-\mathrm{C}_{\mathrm{x}}$ monomers on the polymerization kinetics, data fitting using normalized resonance II (2-oxazoline ring vibration) as input has been performed for the systems containing FDCAox. The reaction kinetics of the polymerization between bis(2-oxazoline)s and dicarboxylic acids can be described using differential equations (2.1) - (2.3):

$$
\begin{aligned}
& \frac{\delta[\text { Acid }]}{\delta t}=-\frac{\delta[\text { Ester }]}{\delta t}=-\frac{\delta[\text { Sec.Amide }]}{\delta t}=-k_{1}[\text { Acid }][0 X] \\
& \frac{\delta[0 X]}{\delta t}=-k_{1}[\text { Acid }][O X]-k_{2}[\text { Sec. Amide }][0 X \\
& \frac{\delta[\text { Tert.Amide }]}{\delta t}=\mathbf{k}_{2}[\text { Sec. Amide }][0 X]
\end{aligned}
$$

In differential equations (2.1) - (2.3), the 2-oxazoline concentration is denoted as $[\mathrm{OX}]$ in $\mathrm{mol} / \mathrm{kg}$. Similarly, the concentrations of the ester, amide groups, and tertiary amide groups are denoted as [Ester], [Sec. Amide], and [Tert. Amide] 
respectively (in mol/kg). After normalization of the area of resonance II by the area of resonance $\mathbf{I}$, followed by conversion to concentration using a conversion factor ${ }^{57}$, the 2-oxazoline concentration during polymerization is obtained. This data has been used as input to fit both $k_{1}$ and $k_{2}$ reaction constants using differential equations (2.1) - (2.3) (Figure 2.9). For the data fitting we assumed that both reactions are second order and irreversible, and that both 2-oxazoline moieties in a bis(2oxazoline) reactant are equireactive ${ }^{52}$. Additionally, we assumed that the 2-oxazoline concentration is linearly dependent on the normalized resonance II peak area, thus ignoring contributions from potential evaporation or changes in density during polymerization $^{57}$.
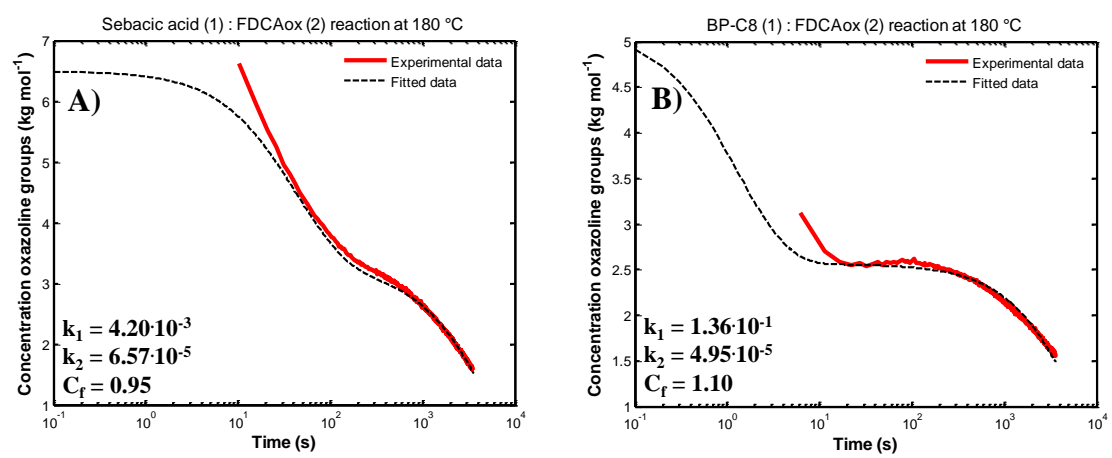

Figure 2.9. Bulk 2-oxazoline concentration during polymerization of (A) Sebacic acid : FDCAox and (B) BP-C $C_{8}$ :FDCAox resins, both in $1: 2$ molar ratios. The red line indicates the experimental data, whereas the black dotted line corresponds to the obtained fit. $C_{f}$ indicates the conversion factor.

From Figure 2.9 we can observe that the used method can fit the FTIR data rather well. Remarkably, the $k_{2}$ reaction constants of the two different resins are the same order of magnitude, while the $k_{1}$ reaction constant increases with more than one order of magnitude when exchanging sebacic acid for $\mathrm{BP}-\mathrm{C}_{8}$. These findings indicate that the previously observed enhancement in reaction and cross-linking rate in $\mathrm{BP}-\mathrm{C}_{8}$ based systems can be attributed to the selective enhancement of the $k_{1}$ reaction constant only. The predicted molecular weight build-up during curing and the resulting decrease in cross-linking time as a function of change in $k_{1}$ reaction constant is provided in Appendix A (Figure A6-A8).

Please note, for the system containing BP- $\mathrm{C}_{8}$, we expect that the $k_{1}$ reaction constant obtained from the fit depicted in Figure 2.9 is overestimated. This is a result from the rapid 2-oxazoline depletion immediately upon the melting of the mixture, thereby preventing the collection of enough data points and at early stage of the 
polymerization. Nevertheless, despite the potential error in the $k_{1}$ value, we can clearly observe that the $k_{1}$ constant is significantly higher when using $\mathrm{BP}-\mathrm{C}_{8}$ as dicarboxylic acid. This behavior is consistently observed in systems containing other BP- $\mathrm{C}_{\mathrm{x}}$ monomers as is shown in Appendix A (Figure A5).

The observed enhancement in $k_{1}$ reaction constant in this work is rather comparable to the findings reported by Néry and coworkers for systems using a pyridine based bis(2-oxazoline). These authors attributed the enhanced reactivity to 1) the basic nature of the pyridine ring and 2) to the stabilization of the protonated 2oxazoline moiety. Given the basic but non-reactive nature of $\mathrm{N}$-alkylated pyrrolidone moeities ${ }^{52}$, it is plausible that they promote the deprotonation of the carboxylic acid moiety and thereby enhance its reactivity towards electrophiles such as 2-oxazolines. Verification of this hypothesis is part of ongoing work and will be communicated in future publications.

\subsubsection{Thermal behavior of the developed thermosets}

With understanding of the effect of $\mathrm{BP}-\mathrm{C}_{\mathrm{x}}$ monomers on the curing kinetics in 2-oxazoline resins, in this section we evaluate their effect on the thermal behavior of the fully renewable thermosets, being systems based on FDCAox. To this end, various resins containing FDCAox and several readily recrystallizable $B P-C_{x}$ monomers have been prepared in equimolar ratio and were cured for one hour at 180 ${ }^{\circ} \mathrm{C}$ in the absence of catalyst. The obtained materials were analyzed for their thermal transitions using DSC analysis. The obtained materials are all amorphous in nature, as can be observed from the exemplary DSC heating and cooling traces for the FDCAox : $\mathrm{BP}_{-} \mathrm{C}_{8}$ based thermoset (Figure 2.10A). As expected, the rigid nature of the pyrrolidone rings in the polymer backbone increases the $T_{\mathrm{g}}\left(70{ }^{\circ} \mathrm{C}\right.$ for $\left.\mathrm{BP}-\mathrm{C}_{8}\right)$ compared to the thermosets based on sebacic acid $\left(52{ }^{\circ} \mathrm{C}\right)$. Furthermore, also in line with expectations, the $T_{\mathrm{g}}$ of the thermoset increases with decreasing number of methylene spacers of the used BP- $\mathrm{C}_{\mathrm{x}}$ monomer (Figure 2.10B).

One particular point for attention in these amorphous materials is that the presence of numerous free electron pairs in the oxygen and nitrogen atoms results in the significant absorption of water: As is visible from Figure 2.10A, exposure to water or moisture results in a plasticizing effect, thereby decreasing the glass transition temperature by roughly $30^{\circ} \mathrm{C}$. Such amorphous behavior combined with the plasticizing effect of water is observed for all FDCAox : BP-C $\mathrm{C}_{\mathrm{x}}$ based thermosets, as is shown in Figure 2.10B. The introduction of a water as plasticizer improves the deformability of the thermoset, but can also result in rapid hydrolysis of the ester groups present in these poly(ester-amide)s ${ }^{11,17,54}$. Though such hydrolysis can hamper 
the structural integrity and lifetime of the materials, it also opens up possibilities for depolymerization and biodegradation of the thermosets.
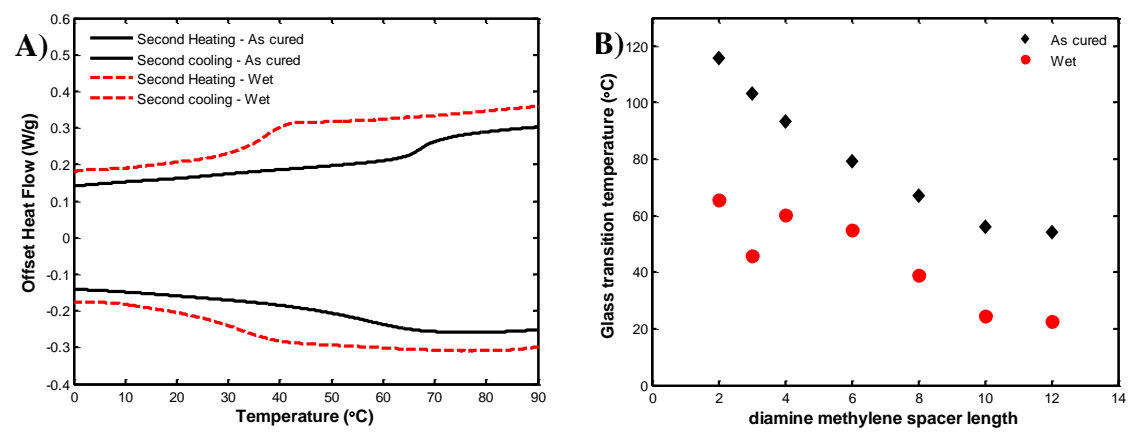

Figure 2.10. A) Second DSC heating and cooling traces for FDCAox: BP-C8 systems cured in an equimolar ratio at $180{ }^{\circ} \mathrm{C}$. The same film has been measured directly after curing and after exposure to air. Image B) depicts the glass transition before and after air exposure for cured films containing various BP-Cx monomers and FDCAox. All DSC runs were performed at heating and cooling rates of $10{ }^{\circ} \mathrm{C} / \mathrm{min}$.

\subsubsection{Enzymatic depolymerization: Towards predicting end of life}

To identify whether such depolymerization occurs in the presence of water, preliminary degradation studies were performed on the synthesized renewable pyrrolidone based polymers, as shown in scheme 2.4 .

In general, $30 \mathrm{mg}$ of product was placed in $1 \mathrm{~mL}$ of demineralized water or tris(hydroxymethyl)aminomethane (tris) buffer (100mM, pH8). Optionally, protease enzyme from Bacillus Sp58 (0.8 ml, 16units/ml, Sigma Aldrich) was added together with $200 \mu \mathrm{L}$ of $0.01 \mathrm{M} \mathrm{CaCl} 2$ solution. Please note, this enzyme is commonly present in soil and is generally responsible for the breakdown of amide bonds. Next, the vials were sealed, and incubated for 72 hours at $50{ }^{\circ} \mathrm{C}$ under constant shaking.

In general, we can observe from Figure 2.11A that both pure water and tris buffer do not absorb UV light in the evaluated range of 250 to $600 \mathrm{~nm}$. BHFDA, the compound expected to be formed after hydrolysis of the ester bonds in FDCAox based thermosets (Scheme 2.4) does display a strong absorption peak around $280 \mathrm{~nm}$. The other hydrolysis product, $\mathrm{BP}-\mathrm{C}_{8}$, displays a minor absorption peak below 320 $\mathrm{nm}$. Ethanolamine, a reaction product expected to be formed after hydrolysis of the furan dicarboxamide bonds displays an adsorption maximum at $260 \mathrm{~nm}$. Lastly, we observe that also the enzyme does not interfere with the TNBS activity as it only displays an absorption maximum below $300 \mathrm{~nm}$. 


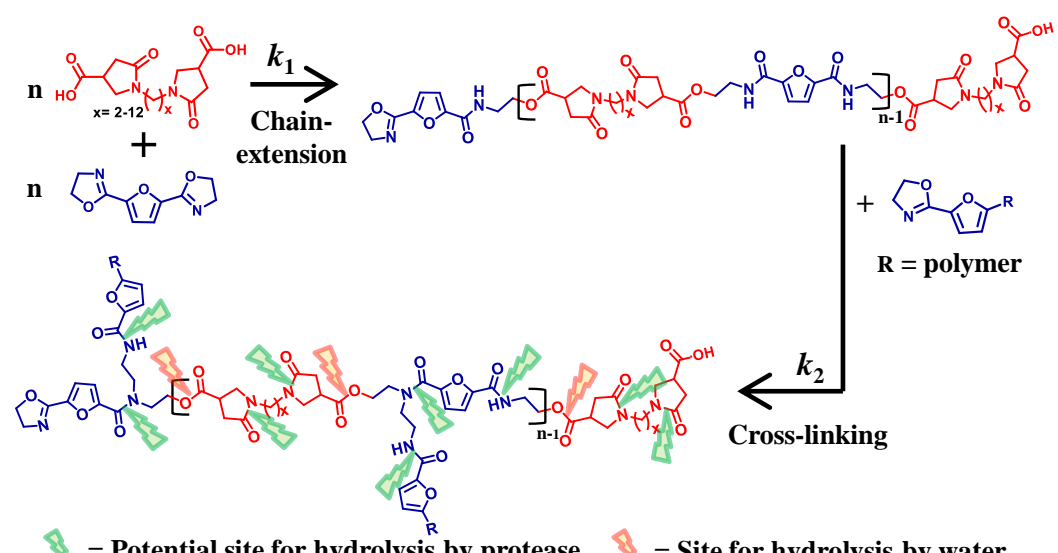

$=$ Potential site for hydrolysis by protease $\&$ = Site for hydrolysis by water

Scheme 2.4: Chain-extension $\left(k_{1}\right)$ and cross-linking $\left(k_{2}\right)$ reaction between renewable dicarboxylic acids and a 2,5-furandicarboxylic acid based bis(2-oxazoline) yielding fully renewable and enzymatically depolymerizable thermosets.

After incubation, the solutions were placed in a cuvette and analyzed using UV-VIS spectrophotometry, together with a series of model compounds expected to form after hydrolysis of the ester bonds in the FDCAox:BP-C 8 (1:1) polymers (Scheme 2.5). Directly after UV-VIS analysis of the mixtures, $25 \mu \mathrm{l}$ of a $1 \mathrm{wt} \%$ solution of 1,4,6-trinitrobenzenesulphonic acid (TNBS) sodium salt solution was added, mixed with a pipette, and placed back for UV-VIS analysis. TNBS is a wellknown indicator for primary amines ${ }^{64-68}$ which allows us to identify whether the used protease facilitate amide bond breakage. In general, TNBS (absorption maximum at 250-260 nm) reacts with primary amines to form a Meisenheimer complex (absorption maximum at $420 \mathrm{~nm}$ ), which in turn can react further into a trinitrophenylamine (absorption maximum at $340 \mathrm{~nm}$ ). In the absence of amines partial hydrolysis of TNBS occurs yielding picric acid which displays an absorption maximum at $340 \mathrm{~nm}^{69}$. Figure 2.11 displays the UV-VIS spectra in the range between 250 and $600 \mathrm{~nm}$ for the various mixtures evaluated in this study.

As expected, the addition of TNBS to water only results in hydrolysis of the TNBS resulting in UV absorption corresponding to the presence of picric acid (Figure 2.11B). Similarly, addition of TNBS to BP-C 8 , BHFDA, and the enzyme solutions only result in partial hydrolysis of TNBS. Please note, also tris buffer does not interact with TNBS in our used detection protocol despite the presence of an amine group, which is likely a result from steric hindrance of the three bulky ethanol side groups. In contrast, the system containing ethanolamine rapidly decolors when TNBS is added and displays a dominant UV absorption at $420 \mathrm{~nm}$, indicating the presence of 
the Meisenheimer complex (in fact, the UV absorption at this concentration is close to detection limit).

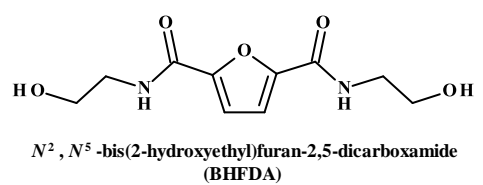

(BHFDA)
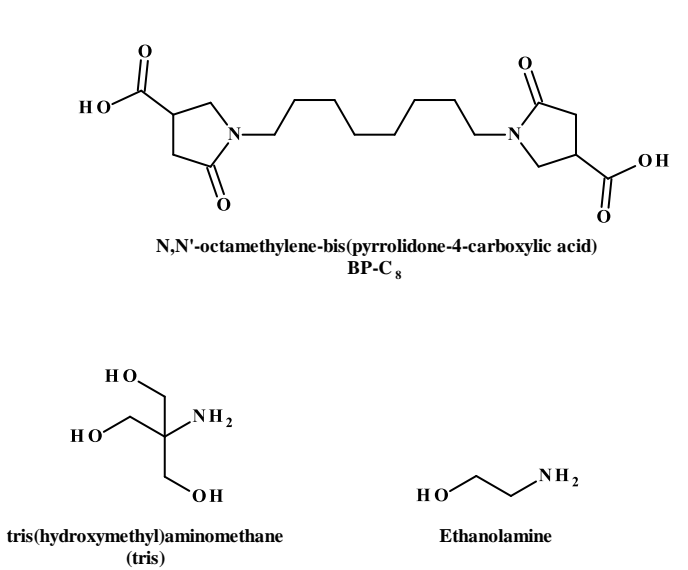

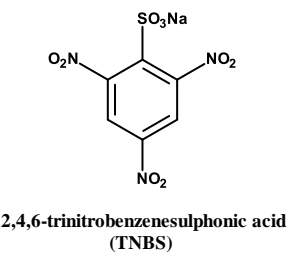
(TNBS)

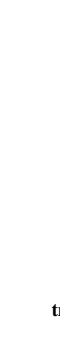

Scheme 2.5. Overview of the reference compounds and the used TNBS sodium salt indicator used to identify UV activity and the presence of amines in the degradation products.

Systems containing FDCAox and $\mathrm{BP}_{-} \mathrm{C}_{8}$ based thermosets (1:1 ratio) were also incubated under various conditions. The general observations from these studies are that the presence of water alone is not sufficient to depolymerize the material: The thermosets immersed in water only swell but do not fully dissolve within a period of several weeks. In contrast, immersion in the slightly alkaline buffer does result in swelling and dissolution of the thermoset within 72 hours. Please note, since the 2,5FDCA based thermosets are crosslinked and insoluble, dissolution of the thermosets is only possible upon chemical break-down. Therefore, the dissolution observed in these degradation studies is likely a result from ester bond hydrolysis and consecutive dissolution of the hydrolyzed products, as has also been reported for BP-C $\mathrm{C}_{\mathrm{x}}$ based polyesters by Miller and coworkers ${ }^{11}$. This therefore indicates that hydrolysis is rather slow in pure water but is enhanced in alkaline conditions. Interestingly, the hydrolysis of the thermoset is significantly enhanced upon the addition of enzyme to polymer in buffer as full dissolution of the thermoset is observed within 48 hours of incubation. Addition of enzyme to polymer in only water does not enhance the hydrolysis rate to such an extent as the polymer merely swells over time. This strongly suggests that the combination of enzyme and buffer facilitates depolymerization of the thermoset through hydrolysis. 

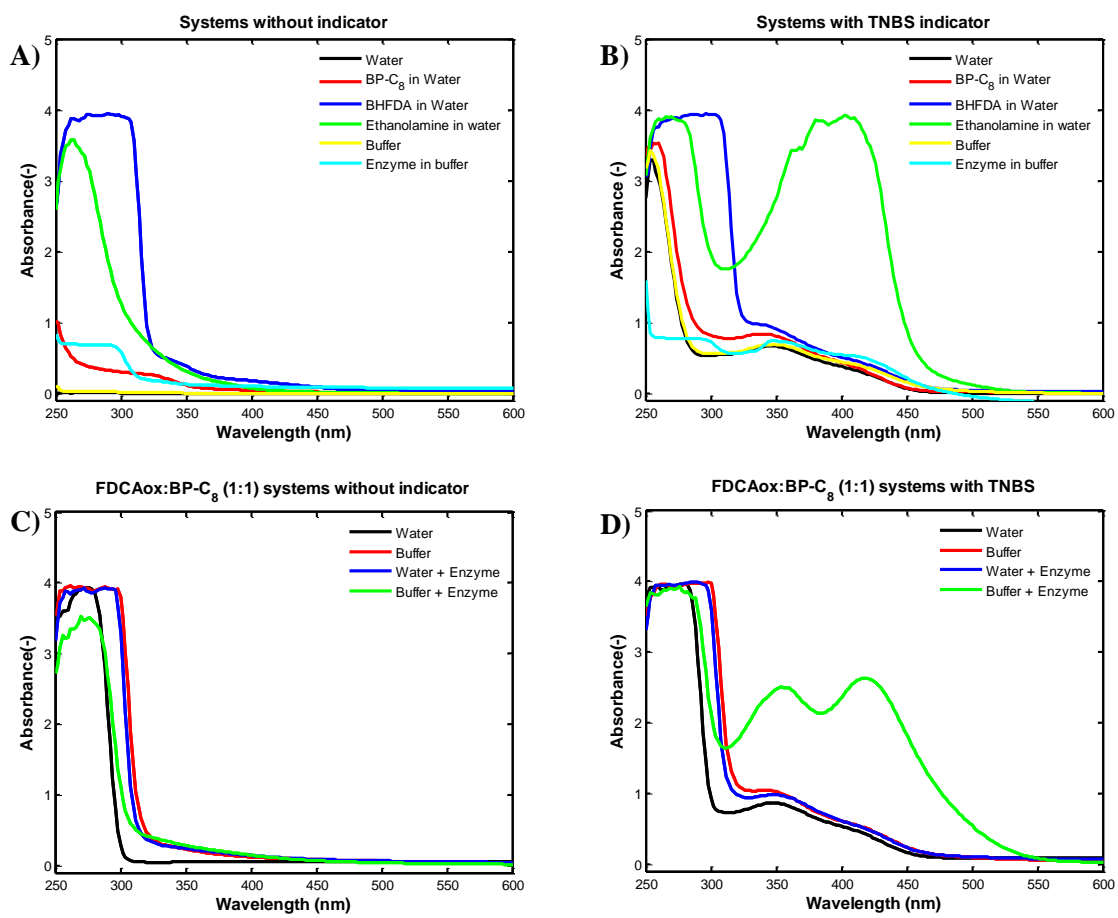

Figure 2.11. UV absorbance of solutions containing various media in the range of 250-600 nm without $(A, C)$ and with $\operatorname{TNBS}(B, D)$ as indicator.

Indeed, this seems to be the case when looking at the UV response of the ensuing mixtures. As is visible from Figure 2.11C, UV absorption is observed for all the samples below $300-320 \mathrm{~nm}$, likely indicating the presence of hydrolysis product BHFDA. No primary amines are detected upon addition of TNBS for the polymers immersed in water, buffer or enzyme solution in water, indicating that only hydrolysis of the ester bonds takes place under these conditions. Interestingly, addition of TNBS to the system containing both buffer and enzyme results in a rapid discoloration of the solution and a rise in UV absorption maximum at 420 . These preliminary findings indicate that the enzyme can also break down the amide bonds, thereby producing primary amines.

Though this absorption of water and consecutive enzymatic depolymerization shows potential for biodegradability of the material, it may be desired to increase the lifetime of the thermosets in the presence of water. One potential route to control the depolymerization rate is by controlling the cross-linking density of the thermosets: An increase in cross-linking density increases the $T_{\mathrm{g}}$ and decreases the tendency of the thermosets to absorb water. As a result, the 
depolymerization process is expected to slow down and thermosets with controlled degradation rates can be developed.

Detailed studies related to the degradation mechanism and products of the developed thermosets as a function of chemical composition and cross-linking density are currently ongoing and will be part of future communications.

\subsection{Conclusions}

The synthesis of fully renewable (bis)pyrrolidone based dicarboxylic acids was shown to be readily achieved in bulk with only water as catalyst. Furthermore, these materials have shown to be excellent candidates for curing in 2-oxazoline based resins: Both GPC and NMR data indicate that the BP- $\mathrm{C}_{\mathrm{x}}$ monomers improve the reaction rate, thus allowing for faster curing. Additionally, through FTIR characterization we observed that the reaction kinetics in 2-oxazoline based resins can be controlled by a judicious selection of the reactants; the $k_{1}$ reaction constant, responsible for chain extension, can be tailored by the choice of the dicarboxylic acid component, whereas the $k_{2}$ reaction constant, responsible for branching and crosslinking, can be controlled by the choice of the bis(2-oxazoline) reactant. Combined, this provides a toolbox for the development of fully renewable and highly reactive resins with tailored thermal properties. Furthermore, the developed thermosets in this study are readily plasticized by water and are promising candidates for biodegradation as they are susceptible for enzymatic depolymerization.

\section{For further information, please refer to Appendix A.}




\subsection{References}

(1) Gandini, A.; Belgacem, M. N. Furans in Polymer Chemistry. Prog. Polym. Sci. 1997, 22 (6), 1203-1379. https://doi.org/10.1016/S0079-6700(97)00004-X.

(2) Robert, T.; Friebel, S. Itaconic Acid-a Versatile Building Block for Renewable Polyesters with Enhanced Functionality. Green Chem. 2016, 18 (10), 2922-2934.

https://doi.org/10.1039/c6gc00605a.

(3) Winkler, M.; Lacerda, T. M.; Mack, F.; Meier, M. A. R. R. Renewable Polymers from Itaconic Acid by Polycondensation and Ring-Opening-Metathesis Polymerization. Macromolecules 2015, 48 (5), 1398-1403. https://doi.org/10.1021/acs.macromol.5b00052.

(4) Van Putten, R. J.; Van Der Waal, J. C.; De Jong, E.; Rasrendra, C. B.; Heeres, H. J.; De Vries, J. G. Hydroxymethylfurfural, a Versatile Platform Chemical Made from Renewable Resources. Chem. Rev. 2013, 113 (3), 1499-1597. https://doi.org/10.1021/cr300182k.

(5) Wang, J.; Liu, X.; Zhang, Y.; Liu, F.; Zhu, J. Modification of Poly(Ethylene 2,5Furandicarboxylate) with 1,4-Cyclohexanedimethylene: Influence of Composition on Mechanical and Barrier Properties. Polymer (Guildf). 2016, 103, 1-8. https://doi.org/10.1016/j.polymer.2016.09.030.

(6) Deng, J.; Liu, X.; Li, C.; Jiang, Y.; Zhu, J. Synthesis and Properties of a Bio-Based Epoxy Resin from 2,5-Furandicarboxylic Acid (FDCA). RSC Adv. 2015, 5 (21), 15930-15939. https://doi.org/10.1039/c5ra00242g.

(7) Dai, J.; Ma, S.; Wu, Y.; Han, L.; Zhang, L.; Zhu, J.; Liu, X. Polyesters Derived from Itaconic Acid for the Properties and Bio-Based Content Enhancement of Soybean Oil-Based Thermosets. Green Chem. 2015, 17 (4), 2383-2392. https://doi.org/10.1039/c4gc02057j.

(8) Dai, J.; Ma, S.; Teng, N.; Dai, X.; Shen, X.; Wang, S.; Liu, X.; Zhu, J. 2,5-Furandicarboxylic Acid- and Itaconic Acid-Derived Fully Biobased Unsaturated Polyesters and Their CrossLinked Networks. Ind. Eng. Chem. Res. 2017, 56 (10), 2650-2657. https://doi.org/10.1021/acs.iecr.7b00049.

(9) Seppälä, J. V.; Helminen, A. O.; Korhonen, H. Degradable Polyesters through Chain Linking for Packaging and Biomedical Applications. Macromol. Biosci. 2004, 4 (3), 208-217. https://doi.org/10.1002/mabi.200300105.

(10) Cowie, J. M. G.; Haq, Z. Poly(Mono n-Alkyl Itaconic Acid Esters): Their Preparation and Some Physical Properties. Br. Polym. J. 1977, 9 (3), 241-245. https://doi.org/10.1002/pi.4980090314.

(11) Qi, P.; Chen, H.-L.; Nguyen, H. T. H.; Lin, C.-C.; Miller, S. A. Synthesis of Biorenewable and Water-Degradable Polylactam Esters from Itaconic Acid. Green Chem. 2016, 18 (15), 41704175. https://doi.org/10.1039/C6GC01081D.

(12) Dai, J.; Ma, S.; Liu, X.; Han, L.; Wu, Y.; Dai, X.; Zhu, J. Synthesis of Bio-Based Unsaturated Polyester Resins and Their Application in Waterborne UV-Curable Coatings. Prog. Org. Coatings 2015, 78, 49-54. https://doi.org/10.1016/j.porgcoat.2014.10.007. 
(13) Farmer, T. J.; Castle, R. L.; Clark, J. H.; Macquarrie, D. J. Synthesis of Unsaturated Polyester Resins from Various Bio-Derived Platform Molecules. Int. J. Mol. Sci. 2015.

https://doi.org/10.3390/ijms160714912.

(14) Ali, M. A.; Tateyama, S.; Oka, Y.; Kaneko, D.; Okajima, M. K.; Kaneko, T. Syntheses of High-Performance Biopolyamides Derived from Itaconic Acid and Their Environmental Corrosion. Macromolecules 2013, 46 (10), 3719-3725. https://doi.org/10.1021/ma400395b.

(15) Ayadi, F.; Mamzed, S.; Portella, C.; Dole, P. Synthesis of Bis(Pyrrolidone-4-Carboxylic Acid)Based Polyamides Derived from Renewable Itaconic Acid - Application as a Compatibilizer in Biopolymer Blends. Polym. J. 2013, 45 (10), 766-774. https://doi.org/10.1038/pj.2012.206.

(16) Chau, N.; Matsuda, S.; Iwakura, Y. Synthesis of Polyamides from Esteramide-diamines. Die Makromol. Chemie 1979, 180 (6), 1435-1440. https://doi.org/10.1002/macp.1979.021800604.

(17) Wang, Z.; Wei, T.; Xue, X.; He, M.; Xue, J.; Song, M.; Wu, S.; Kang, H.; Zhang, L.; Jia, Q. Synthesis of Fully Bio-Based Polyamides with Tunable Properties by Employing Itaconic Acid. Polym. (United Kingdom) 2014, 55 (19), 4846-4856.

https://doi.org/10.1016/j.polymer.2014.07.034.

(18) Lv, A.; Li, Z. L. C. Z.-C.; Du, F. S.; Li, Z. L. C. Z.-C. Synthesis, Functionalization, and Controlled Degradation of High Molecular Weight Polyester from Itaconic Acid via ADMET Polymerization. Macromolecules 2014, 47 (22), 7707-7716.

https://doi.org/10.1021/ma5020066.

(19) Goerz, O.; Ritter, H. Polymers with Shape Memory Effect from Renewable Resources: Crosslinking of Polyesters Based on Isosorbide, Itaconic Acid and Succinic Acid. Polym. Int. 2013, 62 (5), 709-712. https://doi.org/10.1002/pi.4443.

(20) Gao, C.; Wang, J.; Han, S.; Hu, Z.; Liu, Y. Copolymerization Modification of Poly (Butylene Itaconate). AIP Conf. Proc. 2017, 1864 (August). https://doi.org/10.1063/1.4993038.

(21) He, M.; Wang, Z.; Wang, R.; Zhang, L.; Jia, Q. Preparation of Bio-Based Polyamide Elastomer by Using Green Plasticizers. Polymers (Basel). 2016, 8 (7).

https://doi.org/10.3390/polym8070257.

(22) Chen, K.-S.; Ku, Y.-A.; Lin, H.-R.; Yan, T.-R.; Sheu, D.-C.; Chen, T.-M.; Lin, F.-H.

Preparation and Characterization of PH Sensitive Poly(N-Vinyl-2-Pyrrolidone/Itaconic Acid) Copolymer Hydrogels. Mater. Chem. Phys. 2005, 91 (2-3), 484-489.

https://doi.org/10.1016/j.matchemphys.2004.12.037.

(23) Coşkun, R. Graft Copolymerization of Itaconic Acid-Methacrylamide Comonomers onto Poly(Ethylene Terephthalate) Fibers. Eur. Polym. J. 2007, 43 (4), 1428-1435.

https://doi.org/10.1016/j.eurpolymj.2007.01.002.

(24) Sabaa, M. W.; Mokhtar, S. M. Chemically Induced Graft Copolymerization of Itaconic Acid onto Cellulose Fibers. Polym. Test. 2002, 21 (3), 337-343. https://doi.org/10.1016/S01429418(01)00094-0.

(25) Ouyang, Q.; Cheng, L.; Wang, H.; Li, K. Mechanism and Kinetics of the Stabilization Reactions of Itaconic Acid-Modified Polyacrylonitrile. Polym. Degrad. Stab. 2008, 93 (8), 
1415-1421. https://doi.org/10.1016/j.polymdegradstab.2008.05.021.

(26) Hoogenboom, R. Poly(2-Oxazoline)s: A Polymer Class with Numerous Potential Applications. Angew. Chemie - Int. Ed. 2009, 48 (43), 7978-7994. https://doi.org/10.1002/anie.200901607.

(27) Hoogenboom, R.; Schlaad, H. Bioinspired Poly(2-Oxazoline)S. Polymers (Basel). 2011, 3 (4), 467-488. https://doi.org/10.3390/polym3010467.

(28) Lava, K.; Verbraeken, B.; Hoogenboom, R. Poly (2-Oxazoline ) s and Click Chemistry : A Versatile Toolbox towards Multi- Functional Polymers. 2015, 65, 1-28.

(29) Sedlacek, O.; Monnery, B. D.; Filippov, S. K.; Hoogenboom, R.; Hruby, M. Poly(2Oxazoline)s - Are They More Advantageous for Biomedical Applications than Other Polymers? Macromol. Rapid Commun. 2012, 33 (19), 1648-1662.

https://doi.org/10.1002/marc.201200453.

(30) Luxenhofer, R.; Han, Y.; Schulz, A.; Tong, J.; He, Z.; Kabanov, A. V; Jordan, R. Poly ( 2 Oxazoline ) s as Polymer Therapeutics. Macromol. Rapid Commun 2012, 33, 1613-1631. https://doi.org/10.1002/marc.201200354.

(31) Luxenhofer, R.; Sahay, G.; Schulz, A.; Alakhova, D.; Bronich, T. K.; Jordan, R.; Kabanov, A. V. Structure-Property Relationship in Cytotoxicity and Cell Uptake of Poly(2-Oxazoline) Amphiphiles. J. Control. Release 2011, 153 (1), 73-82. https://doi.org/10.1016/j.jconrel.2011.04.010.

(32) Luxenhofer, R.; Schulz, A.; Roques, C.; Li, S.; Bronich, T. K.; Batrakova, E. V.; Jordan, R.; Kabanov, A. V. Doubly Amphiphilic Poly(2-Oxazoline)s as High-Capacity Delivery Systems for Hydrophobic Drugs. Biomaterials 2010, 31 (18), 4972-4979.

https://doi.org/10.1016/j.biomaterials.2010.02.057.

(33) Persigehl, P.; Jordan, R.; Nuyken, O. Functionalization of Amphiphilic Poly(2-Oxazoline) Block Copolymers: A Novel Class of Macroligands for Micellar Catalysis †. 2000. https://doi.org/10.1021/ma0007381.

(34) Jordan, R.; West, N.; Ulman, A.; Chou, Y. M.; Nuyken, O. Nanocomposites by SurfaceInitiated Living Cationic Polymerization of 2-Oxazolines on Functionalized Gold Nanoparticles. Macromolecules 2001, 34 (6), 1606-1611. https://doi.org/10.1021/ma001615j.

(35) Culbertson, B. M. Cyclic Imino Ethers in Step-Growth Polymerizations. Prog. Polym. Sci. 2002, 27 (3), 579-626. https://doi.org/10.1016/S0079-6700(01)00053-3.

(36) Kagiya, T.; Narisawa, S.; Maeda, T.; Fukui, K. Ring-Opening Polymerization of 2-Substituted 2-Oxazolines. J. Polym. Sci. Part B Polym. Lett. 1966, 4 (7), 441-445. https://doi.org/10.1002/pol.1966.110040701.

(37) Kagiya, T.; Narisawa, S.; Maeda, T.; Fukui, K. Preparation of a Crystalline Poly(Ester-Amide) by the Polyaddition Reaction of Bisoxazoline and a Dicarboxylic Acid. J. Polym. Sci. Part B Polym. Lett. 1966, 4 (4), 257-260. https://doi.org/10.1002/pol.1966.110040405.

(38) Lustoň, J.; Kronek, J.; Böhme, F. Synthesis and Polymerization Reactions of Cyclic Imino Ethers. i. Ring-Opening Homopolyaddition of AB-Type Hydroxyphenyl-Substituted 2- 
Oxazolines. J. Polym. Sci. Part A Polym. Chem. 2006, 44 (1), 343-355.

https://doi.org/10.1002/pola.21159.

(39) Lustoň, J.; Kronek, J.; Markus, O.; Janigová, I.; Böhme, F. Synthesis and Polymerization Reactions of Cyclic Imino Ethers. 3. Poly(Ester Amide)s of the AA + BB Type on the Basis of 2-Oxazolines. Polym. Adv. Technol. 2007, 18 (2), 165-172. https://doi.org/10.1002/pat.801.

(40) Kangani, C. O.; Kelley, D. E. One Pot Direct Synthesis of Amides or Oxazolines from Carboxylic Acids Using Deoxo-Fluor Reagent. Tetrahedron Lett. 2005, 46 (51), 8917-8920. https://doi.org/10.1016/j.tetlet.2005.10.068.

(41) Kangani, C. O.; Kelley, D. E.; Day, B. W. One Pot Direct Synthesis of Oxazolines, Benzoxazoles, and Oxadiazoles from Carboxylic Acids Using the Deoxo-Fluor Reagent. Tetrahedron Lett. 2006, 47 (37), 6497-6499. https://doi.org/10.1016/j.tetlet.2006.07.032.

(42) Kempe, K. Screening the Synthesis of 2-Substituted-2- Oxazolines. J. Comb. Chem. Chem. 2009, 11 (July), 274-280. https://doi.org/10.1021/cc800174d.

(43) Pirrung, M. C.; Tumey, L. N. Oxazoline Synthesis from Hydroxyamides by Resin Capture and Ring-Forming Release. J. Comb. Chem. 2000, 2 (6), 675-680.

https://doi.org/10.1021/cc000047g.

(44) Wipf, P.; Venkatraman, S. From Aziridines to Oxazolines and Thiazolines: The Heterocyclic Route to Thiangazole. Synlett 2004, 1 (01), 1-10. https://doi.org/10.1055/s-1997-681.

(45) Cwik, A.; Hell, Z.; Heged??s, A.; Finta, Z.; Horv??th, Z. A Simple Synthesis of 2-Substituted Oxazolines and Oxazines. Tetrahedron Lett. 2002, 43 (22), 3985-3987. https://doi.org/10.1016/S0040-4039(02)00723-2.

(46) Bodner, T.; Ellmaier, L.; Schenk, V.; Albering, J.; Wiesbrock, F. Delocalized $\pi$-Electrons in 2Oxazoline Rings Resulting in Negatively Charged Nitrogen Atoms: Revealing the Selectivity during the Initiation of Cationic Ring-Opening Polymerizations. Polym. Int. 2011, 60 (8), 1173-1179. https://doi.org/10.1002/pi.3126.

(47) Böhme, F.; Clausnitzer, C.; Gruber, F.; Grutke, S.; Huber, T.; Pötschke, P.; Voit, B. Hyperbranched Poly(Ether Amide)s via Nucleophilic Ring Opening Reaction of Oxazolines. High Perform. Polym. 2001, 13 (2). https://doi.org/10.1088/0954-0083/13/2/303.

(48) Huber, T.; Böhme, F.; Komber, H.; Kronek, J.; Luston, J.; Voigt, D.; Voit, B. New Hyperbranched Poly(Ether Amide)s via Nucleophilic Ring Opening of 2-OxazolineContaining Monomers. Macromol. Chem. Phys. 1999, 200 (1), 126-133. https://doi.org/10.1002/(SICI)1521-3935(19990101)200:1<126::AID-MACP126>3.0.CO;2-O.

(49) Jakisch, L.; Bo, F.; Komber, H.; Pompe, G. Synthesis and Thermal Polymerization of Aromatic 2-Oxazolines Containing Carboxylic Groups. 1999, 260, 256-260.

(50) Sano, Y. Polymerization of Bis(2-oxazoline) Compounds with Dicarboxylic Acids. J. Polym. Sci. Part A Polym. Chem. 1989, 27 (8), 2749-2760.

https://doi.org/10.1002/pola.1989.080270820.

(51) Sano, Y.; Arita, K.; Masuda, I. Cross-Linked Polyesteramide from Bis (2-Oxazoline). Google 
Patents October 2, 1984.

(52) Néry, L.; Lefebvre, H.; Fradet, A. Kinetic and Mechanistic Studies of Carboxylic AcidBisoxazoline Chain-Coupling Reactions. Macromol. Chem. Phys. 2003, 204 (14), 1755-1764. https://doi.org/10.1002/macp.200350036.

(53) Tuominen, J.; Seppälä, J. V. Synthesis and Characterization of Lactic Acid Based Poly(EsterAmide). Macromolecules 2000, 33 (10), 3530-3535. https://doi.org/10.1021/ma9916761.

(54) Tarvainen, T.; Karjalainen, T.; Malin, M.; Pohjolainen, S.; Tuominen, J.; Seppälä, J.; Järvinen, K. Degradation of and Drug Release from a Novel 2,2-Bis(2-Oxazoline) Linked Poly(Lactic Acid) Polymer. J. Control. Release 2002, 81 (3), 251-261. https://doi.org/10.1016/S01683659(02)00081-0.

(55) Kylmä, J.; Tuominen, J.; Helminen, A.; Seppälä, J. Chain Extending of Lactic Acid Oligomers. Effect of 2,2'-Bis(2-Oxazoline) on 1,6-Hexamethylene Diisocyanate Linking Reaction. Polymer (Guildf). 2001, 42 (8), 3333-3343. https://doi.org/10.1016/S0032-3861(00)00751-5.

(56) Lustoň, J.; Kronek, J.; Böhme, F.; Komber, H. Azo-group Labelled Polyesters by End-capping with 2-oxazoline Derivatives - Preparation. Macromol. Symp. 2001, 164 (1), 105-116. https://doi.org/10.1002/1521-3900(200102)164:1<105::AID-MASY105>3.0.CO;2-H.

(57) Wilsens, C. H. R. M.; Wullems, N. J. M.; Gubbels, E.; Yao, Y.; Rastogi, S.; Noordover, B. A. J. Synthesis, Kinetics, and Characterization of Bio-Based Thermosets Obtained through Polymerization of a 2,5-Furandicarboxylic Acid-Based Bis(2-Oxazoline) with Sebacic Acid. Polym. Chem. 2015, 6 (14), 2707-2716. https://doi.org/10.1039/c4py01609b.

(58) Gashti, M. P.; Assefipour, R.; Kiumarsi, A.; Gashti, M. P. Enzymatic Surface Hydrolysis of Polyamide 6,6 with Mixtures of Proteolytic and Lipolytic Enzymes. Prep. Biochem. Biotechnol. 2013, 43 (8), 798-814. https://doi.org/10.1080/10826068.2013.805623.

(59) Silva, C.; Araújo, R.; Casal, M.; Gübitz, G. M.; Cavaco-Paulo, A. Influence of Mechanical Agitation on Cutinases and Protease Activity towards Polyamide Substrates. Enzyme Microb. Technol. 2007, 40 (7), 1678-1685. https://doi.org/10.1016/j.enzmictec.2006.09.001.

(60) Song, A. R.; Kim, H. R. Effectiveness of Flavourzyme Treatment on Polyamide Fabric. Fibers Polym. 2013, 14 (12), 2212-2220. https://doi.org/10.1007/s12221-013-2212-y.

(61) Fazio, M. J. Nucleophilic Ring Opening of 2-Oxazolines with Amines: A Convenient Synthesis for Unsymmetrically Substituted Ethylenediamines. J. Org. Chem. 1984, 49 (25), 4889-4893. https://doi.org/10.1021/jo00199a029.

(62) Cui, X.; Yan, D. Preparation, Characterization and Crystalline Transitions of Odd-Even Polyamides 11,12 and 11,10. Eur. Polym. J. 2005, 41 (4), 863-870.

https://doi.org/10.1016/j.eurpolymj.2004.10.045.

(63) Blumstein, A.; Thomas, O. Odd-Even Effect in Thermotropic Liquid Crystalline 4,4'Dihydroxy-2,2'-Dimethylazoxybenzene-Alkanedioic Acid Polymers. Macromolecules 1982, 15 (5), 1264-1267. https://doi.org/10.1021/ma00233a010.

(64) Satake, K.; Okuyama, T.; Ohashi, M.; Shinoda, T. The Spectrophotometric Determination of 
Amine, Amino Acid and Peptide with 2,4,6- Trinitrobenzene 1-Sulfonic Acid. J. Biochem. 1960, 47 (5), 654-660. https://doi.org/10.1093/oxfordjournals.jbchem.a127107.

(65) Cayot, P.; Tainturier, G. The Quantification of Protein Amino Groups by the Trinitrobenzenesulfonic Acid Method: A Reexamination. Anal. Biochem. 1997, 249 (2), 184 200. https://doi.org/10.1006/abio.1997.2161.

(66) Silva, C.; Cavaco-Paulo, A. Monitoring Biotransformations in Polyamide Fibres. Biocatal. Biotransformation 2004, 22 (5-6), 357-360. https://doi.org/10.1080/10242420400025828.

(67) Spadaro, A. C. C.; Draghetta, W.; Del Lama, S. N.; Camargo, A. C. M.; Greene, L. J. A Convenient Manual Trinitrobenzenesulfonic Acid Method for Monitoring Amino Acids and Peptides in Chromatographic Column Effluents. Anal. Biochem. 1979, 96 (2), 317-321. https://doi.org/10.1016/0003-2697(79)90587-6.

(68) Bubnis, W. A.; Ofner, C. M. The Determination of E\{lunate $\}$-Amino Groups in Soluble and Poorly Soluble Proteinaceous Materials by a Spectrophotometrie Method Using

Trinitrobenzenesulfonic Acid. Anal. Biochem. 1992, 207 (1), 129-133. https://doi.org/10.1016/0003-2697(92)90513-7.

(69) Kiranas, E. R.; Tzouwara-Karayanni, S. M.; Karayannis, M. I. The Reaction of Glutamic Acid and Trinitrobenzenesulfonic Acid - Kinetic Study and Analytical Application. Talanta 1997, 44 (6), 1113-1121. https://doi.org/10.1016/S0039-9140(96)02205-9. 


\section{3 \\ 3}

RENEWABLE (PYRROLIDONE)-BASED THERMOSETS: SYNTHESIS, CHARACTERIZATION, AND POTENTIAL ROUTE FOR THEIR CHEMICAL RECYCLING

Chapter partly based on:

Roy, M.; Wilsens, C. H. R. M.; Leoné, N.; Rastogi, S.

ACS Sustain. Chem. Eng. 2019, 7 (9), 8842-8852.

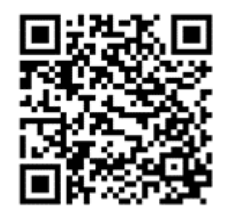




\title{
In this chapter...
}

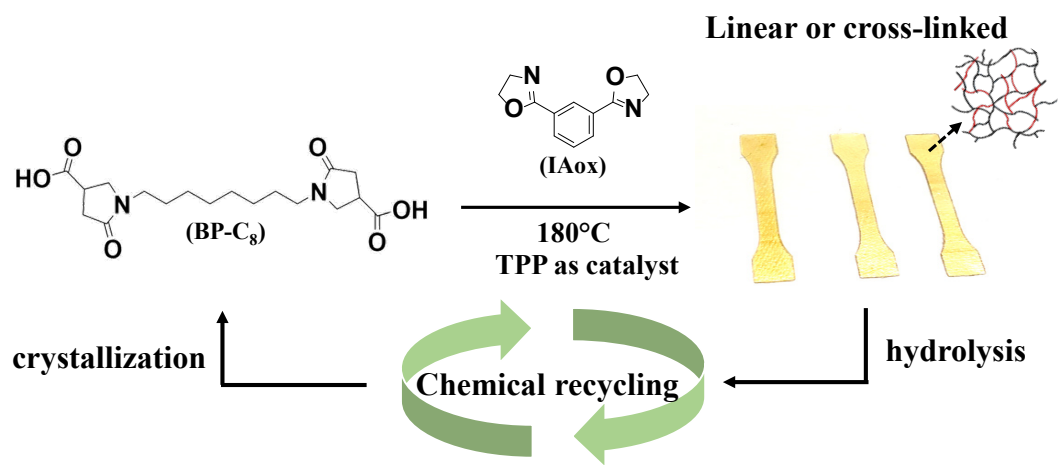

"The synthesis and performance of thermosets using itaconic acid based dicarboxylic acids with bis(2-oxazoline)s is described together with their interaction with moisture, providing a route to recycle the used dicarboxylic acid monomers"

\begin{abstract}
This chapter reports on the synthesis and performance of thermosets using itaconic acid derived (bis)pyrrolidone dicarboxylic acids with bis(2-oxazolines), while investigating their interaction with moisture, providing a route to chemically recycle the used dicarboxylic acid monomers.
\end{abstract}




\subsection{Introduction}

The depletion of fossil feedstock and the usage of traditional plastics have proven to contribute to major environmental issues such as water and air pollution ${ }^{1}$. To this end, industry and academia are exploring routes to make polymers suitable for circular economy, for example through the development of degradable materials or through reuse and recycling of already existing materials ${ }^{2-4}$. Additionally, as a step towards sustainable chemistry ${ }^{5-8}$, bio-based molecules synthesized from renewable feedstock could provide an alternative monomer source for polymers. One of such monomers is itaconic acid ${ }^{9-12}$. The monomer is generally obtained by distillation of citric acid ${ }^{13,14}$ and by fermenting corn $\operatorname{starch}^{15,16}$ which makes it readily available at a low cost. The presence of one unsaturated group along with two carboxylic moieties allows itaconic acid to undergo an aza-Michael addition, thereby generating a pyrrolidone based carboxylic acid functionality. Recently, these monomers have been successfully used for the synthesis of polyesters ${ }^{17}$, polyamides ${ }^{9,10,18-20}$, poly(esteramide) $\mathrm{s}^{21}$, and other polymers ${ }^{22,23}$. In general, the presence of the heterocyclic pyrrolidone ring introduces rigidity into the polymeric backbone, thereby allowing for the development of polymers with enhanced glass transition temperatures. However, the presence of hydrophilic pyrrolidone ring enhances the moisture affinity, effectively facilitating the plasticizing effect and in turn suppressing the glass transition temperature. A concomitant effect of the presence of water is that it facilitates hydrolysis of the pyrrolidone ring, allowing dissolution of the polymers in water and soil, thus making the polymer prone to enzymatic depolymerization ${ }^{21}$ and/or potential biodegradation ${ }^{17}$.

Poly(2-oxazolines) have proven their potential as polymers suitable for biomedical applications ${ }^{24-28}$. These applications have been reported by various researchers such as Hoogenboom et al. ${ }^{29-33}$, Luxenhofer et al. ${ }^{34-36}$, Nuyken et al..$^{37,38}$ and Schubert et al. ${ }^{39-42}$. Additionally, 2-oxazolines are promising candidates in resins for thermal curing with amines ${ }^{43-45}$ and carboxylic acids ${ }^{21,46-50}$.

In previous work, we have demonstrated that the itaconic acid based N,N'octamethylene-bis(pyrrolidone-4-carboxylic acid) $\left(\mathrm{BP}-\mathrm{C}_{8}\right)^{21}$ readily reacted into poly(ester-amide)s at elevated temperatures with both 1,3-bis(4,5-dihydro-2oxazolyl)benzene (IAox) (scheme 3.1, $k_{l}$ reaction) and with the bis(2-oxazoline) derived from renewable 2,5-furandicarboxylic acid. Additionally, when applying an excess of bis(2-oxazoline), the 2-oxazoline functionality can in turn react with the formed amide bonds, thereby generating a tertiary amide group (scheme 3.1, $k_{2}$ reaction). This second reaction proved to be a versatile tool for the development of thermosets, as it allows control over the cross-linking density by merely adding an excess of bis(2-oxazoline). 

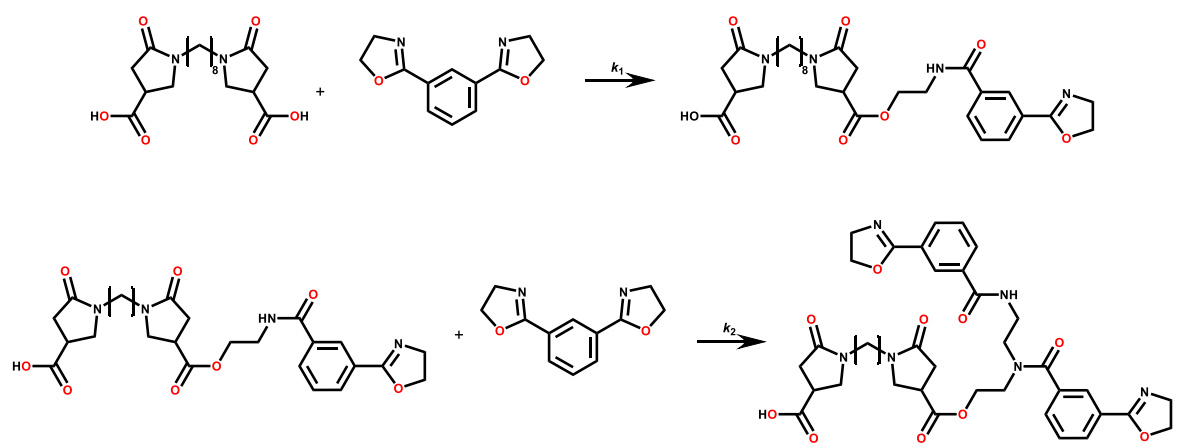

Scheme 3.1. Chain extension $\left(k_{1}\right)$ and branching $\left(k_{2}\right)$ reactions occurring during curing of the resin containing dicarboxylic acid $B P-C_{8}$ and IAox.

When immersed in water, both the ester and amide functionalities in these thermosets are susceptible to enzymatic depolymerization, thereby opening new possibilities for chemical recycling or potential biodegradation ${ }^{21}$. Although these scenarios are promising for the potential circularity of these materials, the actual role of water on the chemical stability, thermal behavior and mechanical behavior of these materials has yet to be identified. Therefore, in this work we report on the role of moisture on the thermo-mechanical behavior of a series of 2-oxazoline based thermosets with varying cross-linking density. Though the use of 2,5furandicarboxylic acid based bis(2-oxazoline) is preferred from renewability aspects, its susceptibility towards cross-linking does not allow for the generation of noncrosslinked polymers which are required as reference in this study. Instead, for a better control over the cross-linking process, in this work we make use of IAox derived from isophthalic acid, which could become a future renewable building block as synthesis routes are emerging with, for example, renewable xylenes ${ }^{51}$, isoprene ${ }^{52}$, or triacylglycerides ${ }^{53}$ as starting compounds. Lastly, in addition to the thermomechanical behavior we report on a potential route for chemical recycling of the BP$\mathrm{C}_{8}$ monomer after hydrolysis of the thermosets.

\subsection{Experimental section}

\subsubsection{Materials}

Itaconic acid (99\% purity), triphenyl phosphite (TPP, 98\% purity), deuterated water $\left(\mathrm{D}_{2} \mathrm{O}, 99\right.$ atoms\% D), and 1,8-diaminooctane (98\% purity) were purchased from Sigma-Aldrich. 1,3-Bis(4,5-dihydro-2-oxazolyl)benzene (IAox, 98\% purity, GC) was purchased from TCI chemicals. The chemicals used are used as received, unless described otherwise. 


\subsubsection{Synthesis of N,N'-octamethylene-bis(pyrrolidone-4-carboxylic acid), $\mathrm{BP}-\mathrm{C}_{8}$}

$\mathrm{BP}_{-} \mathrm{C}_{8}$ used in this study abbreviated as, N,N'-octamethylenebis(pyrrolidone-4-carboxylic acid) was synthesized using the method reported in earlier work (scheme 3.2) ${ }^{21}$ : Itaconic acid $(13.01 \mathrm{~g}, 0.10 \mathrm{~mol})$ and 1,8-octanediamine $(7.21 \mathrm{~g}, 0.05 \mathrm{~mol})$ were placed in a round-bottom flask and heated in bulk to $180{ }^{\circ} \mathrm{C}$ for 18 hours. The product was obtained as off-white crystals via recrystallization from methanol and ethyl acetate and was isolated by filtration followed by drying in vacuo at $80{ }^{\circ} \mathrm{C}$ with a yield of $89 \%$. Purity $(\geq 99 \%)$ of the monomer was determined using NMR analysis. ${ }^{1} \mathrm{H}$ NMR $\left(\mathrm{CDCl}_{3}+\mathrm{d}-\mathrm{TFA}, 300 \mathrm{MHz}\right): \delta 3.80(\mathrm{~m}, 4 \mathrm{H}), 3.44(\mathrm{~m}, 4 \mathrm{H})$, $3.31(\mathrm{~m}, 2 \mathrm{H}), 2.99(\mathrm{~d}, 4 \mathrm{H}, \mathrm{J}=7.9 \mathrm{~Hz}), 1.57(\mathrm{~m}, 4 \mathrm{H}), 1.30(\mathrm{~m}, 8 \mathrm{H}) .{ }^{13} \mathrm{C} \mathrm{NMR}\left(\mathrm{CDCl}_{3}\right.$ + d-TFA, $300 \mathrm{MHz}): \delta 178.2(\mathrm{C}=\mathrm{OOH}), 175.0(\mathrm{NC}=\mathrm{O}), 49.7\left(\mathrm{NCH}_{2} \operatorname{ring}\right), 43.4$ $\left(\mathrm{NCH}_{2}\right.$ spacer), 35.7 ( $\mathrm{CH}$ ring), $33.8\left(\mathrm{CH}_{2}\right.$ ring), $28.6\left(\mathrm{CH}_{2}\right.$ spacer $), 26.5\left(\mathrm{CH}_{2}-\right.$ spacer), $26.1\left(\mathrm{CH}_{2}\right.$ spacer $)$.

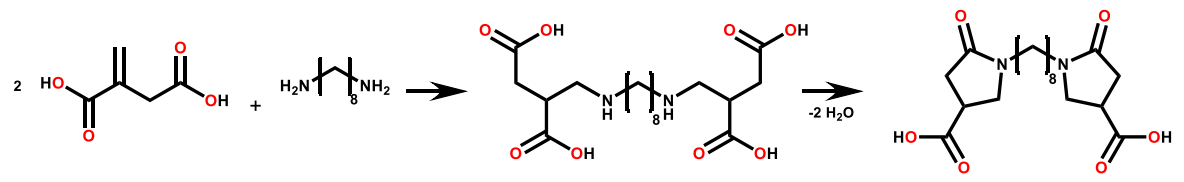

Scheme 3.2: Reaction scheme showing the synthesis of $N, N$ '-octamethylenebis(pyrrolidone-4-carboxylic acid), BP-C8.

\subsubsection{Melt curing procedure}

The desired amount of the BP-C 8 and IAox was weighed together with 1 wt $\%$ triphenyl phosphite. The ensuing product was mixed with a mortar and pestle before usage. The resulting mixture was cured in a compression mold (30 bar pressure) at $180^{\circ} \mathrm{C}$ for 90 minutes to ensure full conversion. The resulting films were dried overnight in vacuo at $70{ }^{\circ} \mathrm{C}$ prior to further use. A series of five polymers, having varying crosslinking density, were synthesized following the given procedure. The crosslinking density was varied by the addition of an excess of IAox. The polymers having an equimolar ratio of $\mathrm{BP}-\mathrm{C}_{8}$ and IAox is named polymer $\mathbf{1}$, whereas the thermosets containing $25 \mathrm{~mol} \%$, $50 \mathrm{~mol} \%$, $75 \mathrm{~mol} \%$, and $100 \mathrm{~mol} \%$ excess of IAox (with respect to $\mathrm{BP}_{-} \mathrm{C}_{8}$ ) are called polymers $\mathbf{2}, \mathbf{3}, \mathbf{4}$, and $\mathbf{5}$, respectively.

\subsubsection{Characterization Methods}

${ }^{1} \mathrm{H}-\mathrm{NMR}$ spectroscopy was performed with a Bruker Ultrashield 300 spectrometer (300 MHz magnetic field). Samples were prepared by dissolving $10 \mathrm{mg}$ 
of material in $0.5 \mathrm{~mL}$ deuterated dimethyl-sulfoxide (DMSO-d6) or deuterated water $\left(\mathrm{D}_{2} \mathrm{O}\right)$. All spectra were referenced against tetramethyl silane (TMS).

Molecular weights of the synthesized poly(ester-amide)s was determined via gel permeation chromatography (GPC). The poly(ester-amide)s (5.0 mg) were dissolved in $1.5 \mathrm{~mL}$ of 1,1,1,3,3,3-hexafluoroisopropanol (HFIP) containing $0.019 \%$ sodium trifluoroacetate. After dissolution, the mixtures were filtered over a $0.2 \mu \mathrm{m}$ PTFE syringe filter before injection. The GPC apparatus was calibrated with poly(methyl methacrylate) standards. Two PFG combination medium microcolumns with $7 \mu \mathrm{m}$ particle size $(4.6 \mathrm{~mm} \times 250 \mathrm{~mm}$, separation range $100-1.000 .000 \mathrm{~g} / \mathrm{mol})$ and precolumn PFG combination medium with $7 \mu \mathrm{m}$ particle size $(4.6 \mathrm{~mm} \times 30 \mathrm{~mm})$ with refractive index detector (RI) were used in order to determine the molecular weight and dispersity.

Attenuated total reflection Fourier transform infrared spectroscopy (ATRFTIR) was performed using a PerkinElmer Spotlight 400 equipped with a PIKE GladiATR, dual mode MCT (mercury cadmium telluride) detector with an array or a temperature-stabilized DTGS (deuterated triglycine sulfate) as a standard configuration. ATR-FTIR spectroscopy carried out on the cured thermosets with various crosslinking densities. Spectra were collected in the range $450-4000 \mathrm{~cm}^{-1}$ with a spectral resolution of $4 \mathrm{~cm}^{-1}$. Polymerization and curing reactions were monitored on-line for two hours at a reaction temperature of $180{ }^{\circ} \mathrm{C}$.

The thermal stability and moisture absorption of the synthesized thermosets were studied using Thermogravimetric analysis (TGA). Experiments were performed on a TA Instruments TGA Q500 in a nitrogen rich atmosphere. Samples were heated from 20 to $500{ }^{\circ} \mathrm{C}$, at a heating rate of $10^{\circ} \mathrm{C} / \mathrm{min}$.

Differential scanning calorimetry (DSC) was performed on a TA Instruments DSC Q2000 to identify the glass transition temperature of the developed materials, both before and after water absorption. Heating and cooling runs were performed at rates of $10^{\circ} \mathrm{C} / \mathrm{min}$. The glass transition temperature of the materials was determined from the first heating run, in order to prevent full evaporation of the water during heating.

Tensile tests were performed to assess the mechanical performance of the thermosets on small scale. To this end, a Linkam micro tensile stage, equipped with a constant force of $50 \mathrm{~N}$ load cell with accuracy of $0.01 \mathrm{~N}$ was used. Samples were cut into a flat dog bone type specimen with an effective gage dimensions of $13 \times 2 \times 1$ $\mathrm{mm}$ (length $\mathrm{x}$ width $\mathrm{x}$ thickness). The tensile tests were performed at a deformation rate of $100 \mathrm{~mm} / \mathrm{min}$. All the measurements were performed at room temperature. 
Torsional DMTA experiments on the inner parts of the PLA tensile bars were performed on an Anton Paar 702 twin drive rheometer equipped with DMTA clamps. The samples were heated from $40{ }^{\circ} \mathrm{C}$ to $160{ }^{\circ} \mathrm{C}$ and exposed to a strain of $1 \%$ and consecutively cooled back to $40{ }^{\circ} \mathrm{C}$.

\subsubsection{Thermoset hydrolysis and monomer recovery tests}

The hydrolysis and monomer recovery tests were performed on the polymer $\mathbf{1}$ and polymer $\mathbf{5}$. Sample specimens were cut and immersed in demineralized water, followed by heating to $130{ }^{\circ} \mathrm{C}$ in a pressure vessel for three or six hours. Next, the supernatant was decanted off, cooled down to room temperature and stored at $5^{\circ} \mathrm{C}$ after addition to methanol as co-solvent. Over time, small crystallites formed which were filtered off, dried at $60{ }^{\circ} \mathrm{C}$ in vacuo prior to analysis using NMR spectroscopy.

\subsection{Results and discussion}

\subsubsection{Synthesis, characterization and thermal behavior of developed thermosets}

As explained in the experimental section, the $\mathrm{BP}_{-} \mathrm{C}_{8}$ monomer was synthesized according to the route reported in previous publication ${ }^{21}$, and recalled briefly in the experimental section. Scheme 3.1 demonstrates the thermal curing of a 2-oxazoline functionality with a dicarboxylic acid group, which results into a ringopening addition reaction yielding an ester-amide moiety ( $k_{1}$ reaction). In turn, the generated amide bonds react with a 2-oxazoline moiety catalyzed by triphenylphosphite (TPP) resulting in the generation of a tertiary amide bond $\left(k_{2}\right.$ reaction). This reaction mechanism, combined with the use of an excess of bis(2oxazoline) with respect to $\mathrm{BP}-\mathrm{C}_{8}$, is employed to control the cross-linking density in our thermosets. To assess the required curing time to achieve full conversion, thermal curing of IAox and $\mathrm{BP}_{-} \mathrm{C}_{8}$ in a molar ratio of $2: 1$ is performed at $180{ }^{\circ} \mathrm{C}$ and monitored on-line using ATR-FTIR. Spectra are collected over time and are used to monitor the depletion of the 2-oxazoline functionality. An overview of the resulting FTIR spectra is provided in Figure 3.1(a), whereas an overlay of the spectra in Figure 3.1(a) is provided in Figure 3.1(b). 

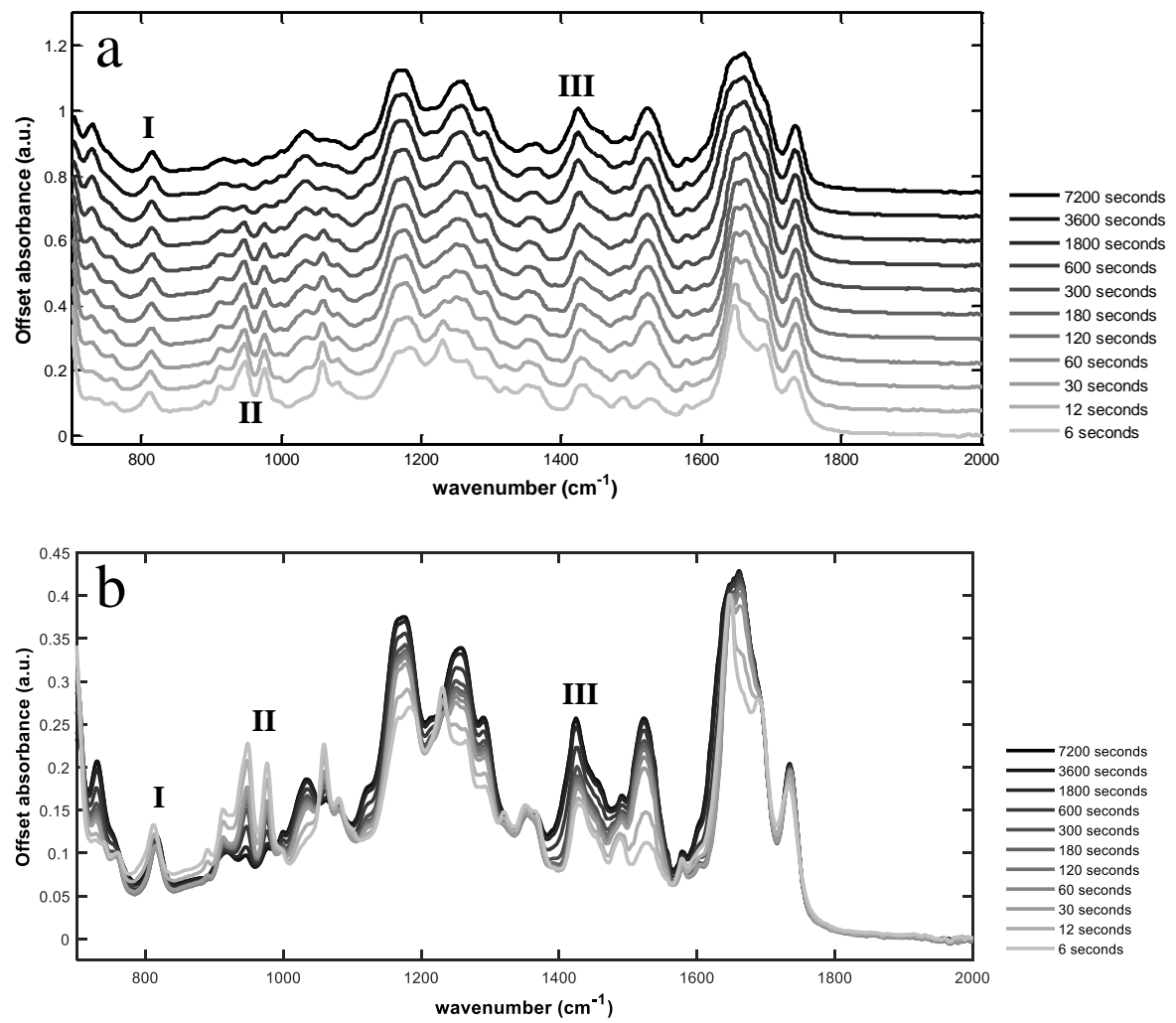

Figure 3.1: (a) FTIR spectra (700-2000 $\mathrm{cm}^{-1}$ ) obtained during thermal curing of IAox and $\mathrm{BP}-\mathrm{C}_{8}$ in a molar ratio of $2: 1$, at $180{ }^{\circ} \mathrm{C}$. Vibrational bands I, II, and III are the characteristic vibration modes arising from the phenyl ring, 2-oxazoline moiety, and the tertiary amide bonds, respectively; (b) Overlay of FTIR spectra $\left(700-2000 \mathrm{~cm}^{-1}\right)$ obtained during thermal curing of IAox and BP-C $C_{8}$ in a molar ratio of $2: 1$, at $180{ }^{\circ} \mathrm{C}$. Vibrational bands $\mathbf{I}, \mathbf{I I}$, and III are the characteristic vibration modes arising from the phenyl ring, 2-oxazoline moiety, and the tertiary amide bonds, respectively.

From Figure 3.1(a) and (b) it is apparent that the two 2-oxazoline ring vibrations ${ }^{21,46}$ decrease over time (vibrations II, between 900 and $1000 \mathrm{~cm}^{-1}$ ), while the vibration of the phenyl rings of IAox (vibration $\mathbf{I}$, at $815 \mathrm{~cm}^{-1}$ ) remains constant. Given that intensity of the signal I remains constant over time, the possibility of monomer evaporation in the system could be excluded. Instead, the decrease in vibrations II confirms the consumption of the 2-oxazoline moieties resulting from to polymerization. Additionally, the occurrence of the $k_{2}$ reaction shown in Scheme 3.1 can be detected by the steady increase in vibration III $\left(1430 \mathrm{~cm}^{-1}\right)$ over time, 
corresponding to the generation of tertiary amide bonds (Figure 3.1(b)). It is to be noted that vibration III is already present at the start of the reaction, resulting from the vibrations of the amide group of the pyrrolidone rings in $\mathrm{BP}_{-} \mathrm{C}_{8}$. Nevertheless, the FTIR experiments confirm that the curing of $\mathrm{BP}_{-} \mathrm{C}_{8}$, with an excess of IAox, can readily be achieved at $180{ }^{\circ} \mathrm{C}$ within one hour in the presence of $1 \mathrm{wt} \% \mathrm{TPP}$.

Thermosets containing BP-C 8 and a various molar excess of IAox $(0 \%, 25 \%$, $50 \%, 75 \%$ and $100 \%$, corresponding to polymer $\mathbf{1}, \mathbf{2}, \mathbf{3}, \mathbf{4}$, and $\mathbf{5}$, respectively) are prepared under the same conditions: $1 \mathrm{wt} \%$ of TPP is added and the monomer mixture is grinded using a mortar and pestle. The ensuing mixtures are thermally cured in bulk at $180{ }^{\circ} \mathrm{C}$ in a compression mold. To confirm the full conversion of 2-oxazoline moieties, FTIR analysis is performed on the products and the resulting spectra are provided in Figure 3.2

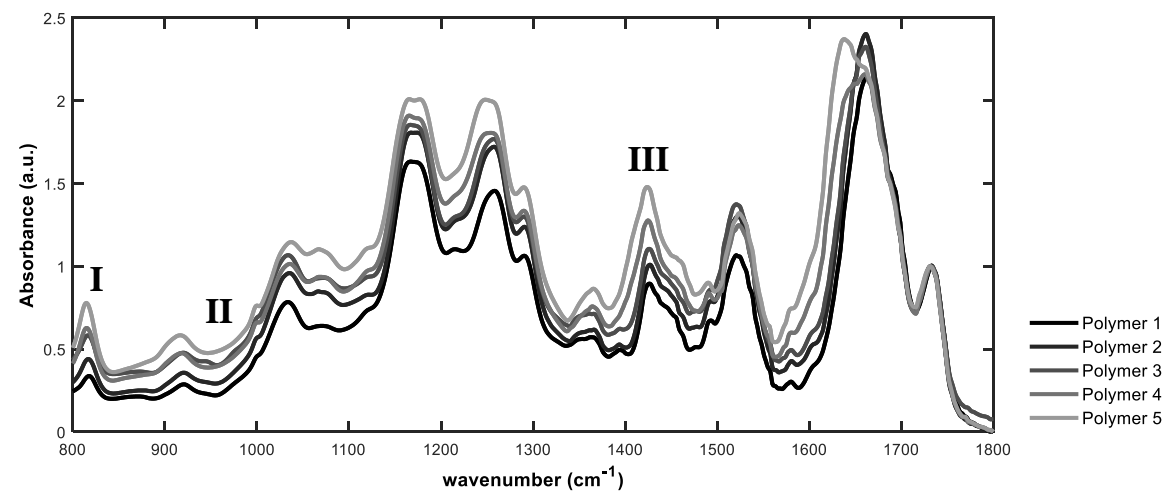

Figure 3.2. FTIR spectra between $800-1800 \mathrm{~cm}^{-1}$ obtained after the curing of BP$C_{8}$ : IAox at $180^{\circ} \mathrm{C}$. The curing was performed on resins having a composition of $B P$ $C_{8}$ : IAox in molar ratio of 1:1 (Polymer 1), 1:1.25 (Polymer 2), 1:1.5 (Polymer 3), 1:1.75 (Polymer 4), and 1:2 (Polymer 5).

To ease comparison, all spectra are normalized against the ester carbonyl stretch vibration at $1734 \mathrm{~cm}^{-1}$. Similar to Figure 3.1, vibrational bands I, II, and III are highlighted in Figure 3.2 and correspond to the vibrations of the aromatic ring, 2oxazoline moiety, and the tertiary amide bonds, respectively. Indeed, no vibrations corresponding to the presence of the 2-oxazoline moiety are detected in any sample, confirming that the samples have achieved a high conversion. Additionally, on plotting the intensity of vibrational band III as a function of molar excess of IAox, it becomes apparent that the signal increases linearly with increasing IAox concentration (Figure 3.3). These observations suggest that the employed excess of 
2-oxazoline moieties is successfully consumed and converted into tertiary amide bonds, effectively increasing the cross-linking density.

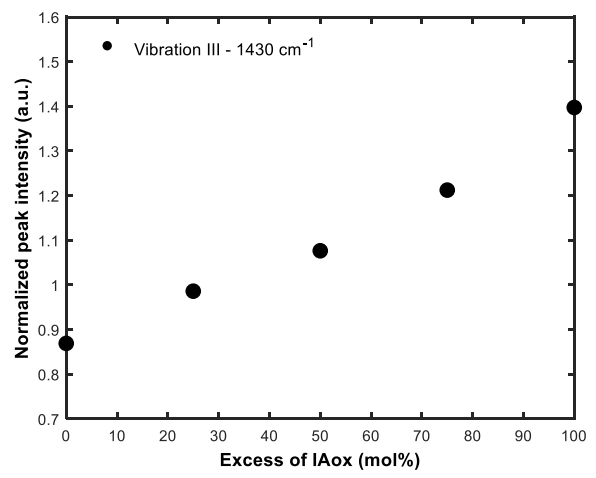

Figure 3.3. Intensity of the vibrational band at $1430 \mathrm{~cm}^{-1}$ as a function of the excess of IAox used during polymerization.

DSC analysis is performed on the thermosets to assess the effect of crosslink density on the glass transition temperature $\left(T_{\mathrm{g}}\right)$. As expected, the $T_{\mathrm{g}}$ of the thermosets gradually increases with the crosslink density: Polymer $\mathbf{1}$ exhibits a $T_{\mathrm{g}}$ of $63{ }^{\circ} \mathrm{C}$, while the $T_{\mathrm{g}}$ of polymer 5 approaches $100{ }^{\circ} \mathrm{C}$ (Figure 3.4 , left).

To assess the influence of water on the glass transition temperature, the thermosets are exposed to different relative humidity $(\mathrm{RH})$ conditions: ambient conditions (approaching 50\% RH in our laboratories), 70\% RH and 90\% RH using a climate chamber. After exposing the materials for a day in these conditions, the $T_{\mathrm{g}}$ is determined using DSC (Figure 3.4, left). The right image of Figure 3.4 displays the characteristic DSC heating curves obtained for the material having an equimolar ratio of BP-C $\mathrm{C}_{8}$ and IAox, directly after curing and after exposure to $70 \%$ and $90 \% \mathrm{RH}$. As anticipated, absorption of moisture facilitates a plasticizing effect, thus causing a suppression in the glass transition temperature. Additionally, we observe that the decrease in $T_{\mathrm{g}}$, resulting from the plasticizing effect of water, is independent of the cross-link density; From Figure 3.4 left it can be observed that the $T_{\mathrm{g}}$ of all thermosets drops roughly by $40{ }^{\circ} \mathrm{C}$ when exposed to ambient conditions, $50{ }^{\circ} \mathrm{C}$ after exposure to $70 \% \mathrm{RH}$, and $70{ }^{\circ} \mathrm{C}$ when exposed to $90 \% \mathrm{RH}$. 

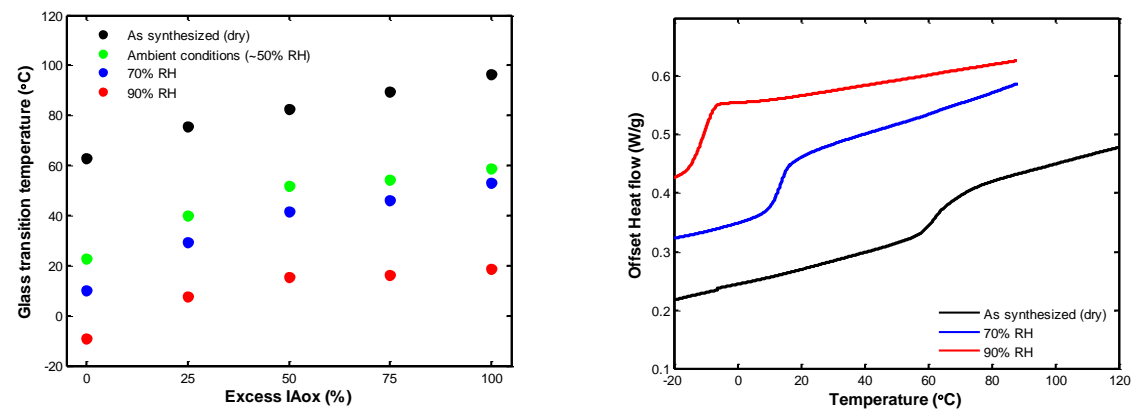

Figure 3.4. Left, thermoset $T_{g}$ as a function of IAox excess used during curing and various humidity conditions. Right, exemplary DSC heating traces depicting the $T_{g}$ suppression of polymer 1 after exposure to different humidity conditions.

The effective $T_{\mathrm{g}}$ suppression can be associated with the total amount of water absorbed during exposure to moisture. To confirm, TGA analysis is performed on the thermosets exposed to different RH (Figure 3.5, left) and with varying excess of IAox used for polymerization (Figure 3.5, right). To assess the maximum amount of absorbed water at room temperature, the polymers are immersed in demineralized water for a day prior to TGA analysis. The dry polymer $\mathbf{1}$ does not display any notable weight loss below $300{ }^{\circ} \mathrm{C}$, after which rapid degradation occurs. However, upon exposure of the material to humidity, one can observe a stepwise weight loss occurring at $100{ }^{\circ} \mathrm{C}$. This stepwise decrease is thought to be the result of diffusion and evaporation of absorbed water. Furthermore, the weight loss increases after exposure to increasing relative humidity. Note that for the sample that was immersed in water at room temperature, the weight loss is observed already below $100{ }^{\circ} \mathrm{C}$. This evaporation at relatively low temperatures is associated with the water adhered to the surface of the sample, instead of water diffused into the sample. Additionally, the amount of absorbed water decreases with increasing cross-linking density, as is highlighted by the arrow in Figure 3.5 right. It is to be noted that prolonged exposure to these humidity conditions does not change the amount of absorbed water. To recall, exposure of the sample to the same conditions for a full week showed the same amount of absorbed water after the TGA analysis. These findings confirm that the water absorption at a given relative humidity is already at equilibrium after one day of exposure. Overall, from these findings we can conclude that the polymers synthesized in this study are highly sensitive to water/moisture. 

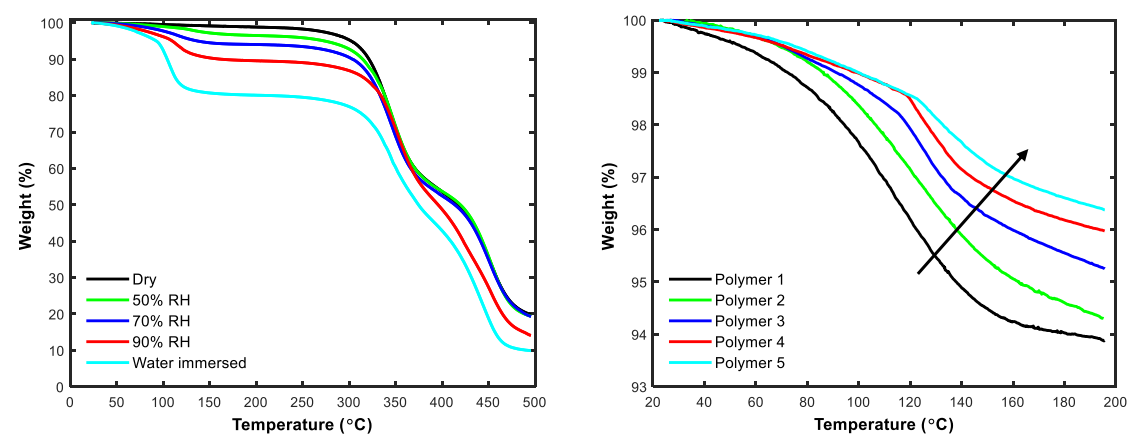

Figure 3.5. Left) Weight loss of the polymer 1 after exposure to various $R H$ conditions, where the ambient conditions approach 50\% RH in our laboratories. Right) Weight loss of polymer $\mathbf{1}$ - 5 after exposure to ambient conditions.

\subsubsection{Effect of moisture on hydrolytic stability and mechanical performance}

To address potential applications of these thermosets, identification of the hydrolytic stability is of key importance. The previously observed tendency of the developed materials to absorb water could pose a problem as this facilitates hydrolysis of the ester bonds next to the $\mathrm{BP}_{-} \mathrm{C}_{8}$ monomer (Scheme 3.1). Such hydrolysis would result in the liberation of oligomers or even $\mathrm{BP}_{-} \mathrm{C}_{8}$ monomers, effectively decreasing the molecular weight of the material and resulting in rapid deterioration of the mechanical properties. Therefore, the hydrolytic stability of the polymer $\mathbf{1}$ is assessed after exposure to the various RH conditions evaluated in this study (Table 3.1, Figure 3.6). This polymer 1, having no excess of IAox is not crosslinked and therefore could be analyzed using GPC techniques $\left(M_{\mathrm{w}}\right.$ after curing was $56 \mathrm{~kg} / \mathrm{mol}$ ). In general, a decrease in $M_{\mathrm{w}}$ is observed upon exposure to increasing $\mathrm{RH}$ and/or upon increasing the exposure time.

Table 3.1: Weight average molecular weight $\left(M_{w}\right)$ obtained after exposure of polymer 1 for 1 or 7 days to the various humidity conditions evaluated in this study. Note, directly after curing, the polymer containing an equimolar ratio of $B P-C_{8}$ and IAox has an $M_{w}$ of $56 \mathrm{~kg} / \mathrm{mol}$.

\begin{tabular}{|ccccc|}
\hline $\begin{array}{c}\text { Exposure } \\
\text { time (days) }\end{array}$ & $\begin{array}{c}\text { Ambient } \\
\text { conditions }\end{array}$ & $\mathbf{7 0 \%}$ R.H. & $\mathbf{9 0 \%}$ R.H. & $\begin{array}{c}\text { Immersed } \\
\text { in water }\end{array}$ \\
\hline $\mathbf{1}$ & $54.3 \mathrm{~kg} / \mathrm{mol}$ & $50.8 \mathrm{~kg} / \mathrm{mol}$ & $50.9 \mathrm{~kg} / \mathrm{mol}$ & $42.5 \mathrm{~kg} / \mathrm{mol}$ \\
\hline $\mathbf{7}$ & $52.2 \mathrm{~kg} / \mathrm{mol}$ & $50.0 \mathrm{~kg} / \mathrm{mol}$ & $48.4 \mathrm{~kg} / \mathrm{mol}$ & $40.9 \mathrm{~kg} / \mathrm{mol}$ \\
\hline
\end{tabular}


Though TGA analysis displayed no difference in the amount of water absorbed over time, the GPC analysis demonstrates that prolonged exposure to moisture does result in a gradual decrease in molecular weight over time.
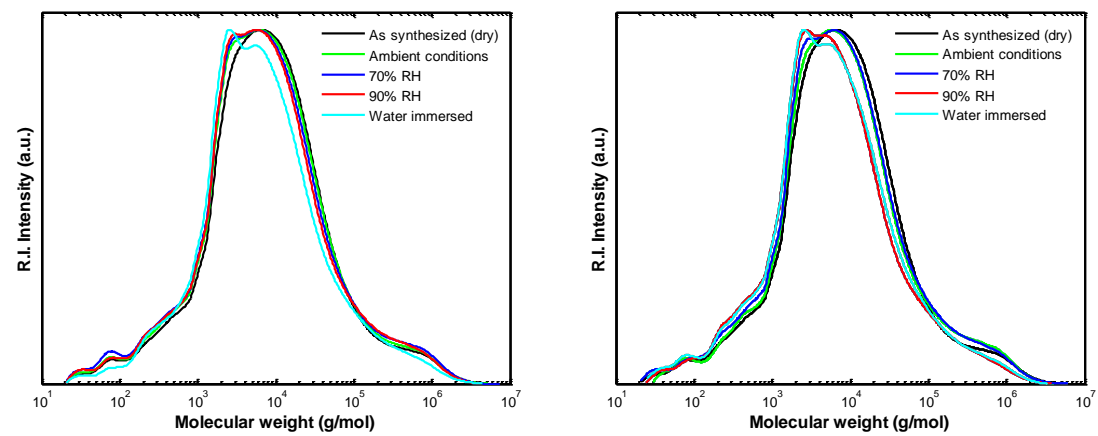

Figure 3.6. GPC traces showing the hydrolysis of polymer 1 after exposure to various climatic conditions: Dry, 50\% RH, 70\% RH, 90\% RH, and immersion in water for one day (left) and one week (right).

Additionally, to the plasticizing effect of water, the decrease in molecular weight as a result of hydrolysis also results in a decrease of the glass transition temperature shown in figure 3.7. From these results it can be concluded that the hydrolysis of $\mathrm{BP}_{-} \mathrm{C}_{8}$ based thermosets with bis(2-oxazolines) is indeed occurring, hence these thermosets could be suitable candidates for applications where controlled degradation is desired.

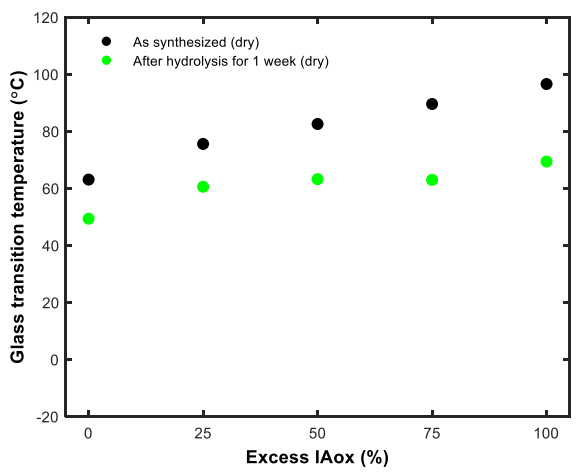

Figure 3.7. $T_{g}$ observed from DSC analysis of the as synthesized polymers (black) and the same polymers (green) after immersion in demineralized water for 7 days. Note, the samples immersed in water are dried in vacuo elevated temperatures to minimize residual water in the sample prior to DSC analysis. 
The mechanical performance of the developed thermosets is evaluated after exposure to moisture for a week. Directly after synthesis, i.e. in the dry state, the thermosets are highly brittle in nature. In fact, we are unable to reproducibly cut tensile bars from cured films without cracking or breaking the thermosets. In contrast, the presence of water and the concomitant suppression in $T_{\mathrm{g}}$ allowed for ease in handling, sample preparation, and repeatable mechanical testing. The characteristic tensile data and standard deviations (average over 3 specimens) of the thermosets after exposure to $70 \% \mathrm{RH}$ are provided in Table 3.2 whereas the characteristic tensile curves are shown in Figure 3.8.

Table 3.2. Young's modulus and maximum stress (either yield or fracture) of the polymers 1 - 5 exposed to 70\% RH. Standard deviations are provided and were averaged over three specimens.

\begin{tabular}{|ccc|}
\hline Polymer & Young's Modulus (GPa) & Fracture stress (MPa) \\
\hline $\mathbf{1}$ & $0.05 \pm 0.02$ & $0.30 \pm 0.08$ \\
\hline $\mathbf{2}$ & $0.23 \pm 0.08$ & $3.26 \pm 0.47$ \\
\hline $\mathbf{3}$ & $0.42 \pm 0.37$ & $21.9 \pm 5.61$ \\
\hline $\mathbf{4}$ & $0.72 \pm 0.06$ & $28.8 \pm 4.94$ \\
\hline $\mathbf{5}$ & $1.28 \pm 0.19$ & $31.5 \pm 2.87$ \\
\hline
\end{tabular}

In general, we observe that the increase in cross-linking density results into thermosets having increasing yield point and a decreasing strain at break. For example, polymer 5 displays a maximum stress of $34 \mathrm{MPa}$ prior to failure, after being exposed to $70 \% \mathrm{RH}$. Upon decreasing the cross-linking density, the materials go through a brittle-to-ductile transition, which is evident from the decrease in tensile modulus, the decrease in yield stress and the increase in strain at break of polymers 4, 3 and 2, respectively (Figure 3.8). Such a decrease in yield point can be correlated to the decreasing $T_{\mathrm{g}}$ of the thermosets resulting from the absorbed water, as is shown in Figure 3.4.

A similar trend is observed for polymer 2 - 5 exposed to $90 \% \mathrm{RH}$; These thermosets displayed rubbery deformation behavior independent of their cross-link density (Figure 3.9). The cause for such rubbery deformation is associated with the large amount of absorbed water, the resulting suppression of the $T_{\mathrm{g}}$ to values below room temperature, and hence the absence of a yield point. 


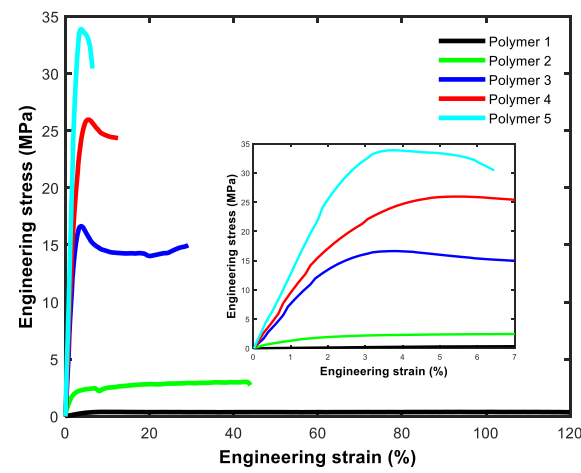

Figure 3.8. Characteristic engineering stress vs engineering strain curves for polymer 1 - 5 after exposure to $70 \%$ RH. Note, the inset displays the same traces, only at low engineering strain.

The only exception to this behavior is polymer 1 , which, due to its $T_{\mathrm{g}}$ below room temperature (Figure 3.4) and the absence of cross-links, flows during the tensile experiments. Overall, these findings stress the importance of moisture in these materials as it facilitates a brittle-to-ductile transition and thereby toughens the thermosets.

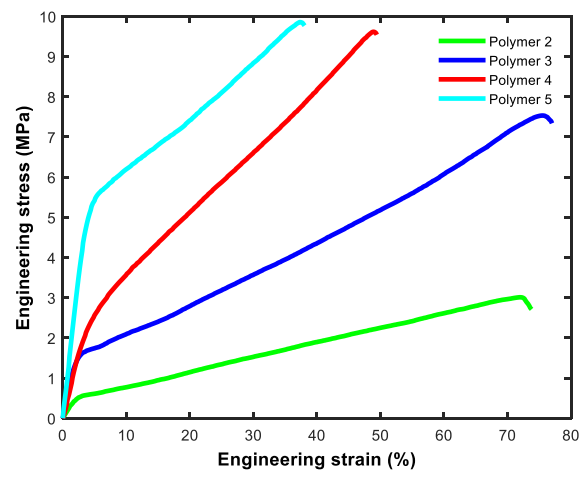

Figure 3.9. Characteristic engineering stress vs engineering strain curves for polymer $2-5$ after exposure to $90 \% \mathrm{RH}$.

\subsubsection{Potential of BP-C $C_{8}$ based polymers as adhesive}

Given the affinity to moisture, we have evaluated the potential of the developed polymers as adhesive for PLA and alumina both under ambient conditions and after immersion in water. Therefore, specimens were prepared as described in the experimental section and subjected to lap shear tests, following the ASTM D 5868 
standard. The tensile data are provided in Figure 3.10 whereas an overview and comparison of data is provided in Tables 3.3 and 3.4.

Table 3.3. Overview of adhesive strength of the different adhesives and polylactide on alumina, conducted under ambient conditions and under water (or after immersion in water, as is done in this work).

\begin{tabular}{|cc|c|}
\hline Adhesive & $\begin{array}{c}\text { Adhesion in } \\
\text { air }(\mathbf{M P a})\end{array}$ & $\begin{array}{c}\text { Adhesion under } \\
\text { water(MPa) }\end{array}$ \\
\hline Elmer's glue (polyvinyl alcohol) $^{\mathbf{A}}$ & $3 \pm 1$ & 0 \\
\hline Gorilla glue (polyurethane) $^{\mathbf{A}}$ & $2.8 \pm 0.7$ & $2.5 \pm 0.8$ \\
\hline Starch glue $^{\mathbf{A}}$ & $2.4 \pm 0.4$ & 0 \\
\hline Hide glue $^{\mathbf{A}}$ & $0.8 \pm 0.1$ & 0 \\
\hline Polylactide $^{\mathbf{A}}$ & $0.21 \pm 0.06$ & $0.10 \pm 0.05$ \\
\hline P1 & $2.3 \pm 0.37$ & $2.0 \pm 0.36$ \\
\hline
\end{tabular}

${ }^{\mathrm{A}}$ Data obtained from J.J. Wilker et al. ${ }^{54}$; The bonds are lap-shear joints between two aluminum pieces cured at $37^{\circ} \mathrm{C}$ for 24 hours, whereas PLA was applied as adhesive through solvent casting.

These preliminary results indicate that the use of $\mathrm{BP}_{-} \mathrm{C}_{8}$ based polymers as adhesive for alumina is highly promising as it can be used under ambient and wet conditions. Overall, the performance of $\mathbf{P 1}$ as adhesive it is slightly inferior to Gorilla glue ${ }^{\circledR}$, though we expect that improvement can be made by optimizing the chemical composition of the polymer.

Table 3.4. Overview of the adhesive strength of polymers having an equimolar ratio of $\mathrm{BP}-\mathrm{C}_{8}$ and IAox, used as adhesive to polylactide. Lab shear tests were performed after exposure to three different humidity conditions; in the dry state, under ambient conditions, and after immersion in water.

\begin{tabular}{|ccc|}
\hline Adhesives composition & $\begin{array}{c}\text { Maximum force } \\
(\mathbf{N})\end{array}$ & $\begin{array}{c}\text { Tensile shear strength } \\
(\mathbf{M P a})\end{array}$ \\
\hline Catechol-PLA bonding $^{\mathbf{A}}$ & - & $0.21+0.006$ \\
\hline P1 (dried) & $90.3+31.3$ & $1.07+0.35$ \\
\hline P1 (ambient condition) & $57.2+3.79$ & $0.73+0.06$ \\
\hline P1 (water immersed) & $49.3+19.3$ & $0.56+0.20$ \\
\hline
\end{tabular}

${ }^{\mathrm{A}}$ Data obtained from J.J Wilker et.al. ${ }^{54}$

Instead, when employing P1 as adhesive for PLA, we observe that the samples fail already at lower force. For comparison, the adhesive strength of P1 with alumina is 2.35 MPa, whereas that with PLA is only around 1.1 MPa. We expect that the strong hydrogen bonding of P1 with the metal surface can facilitate a stronger bonding 
strength. Accordingly, we expect that the decreased capability of PLA to accept hydrogen bonds is a plausible reason for its lowered interaction with P1 (i.e. it only contains one carbonyl per repeat unit as hydrogen bond acceptor). Furthermore, the presence of water proves detrimental for the adhesive strength of P1 to PLA, as is observed from its suppression to from roughly 1.1 to $0.7 \mathrm{MPa}$ (Table 3.4).
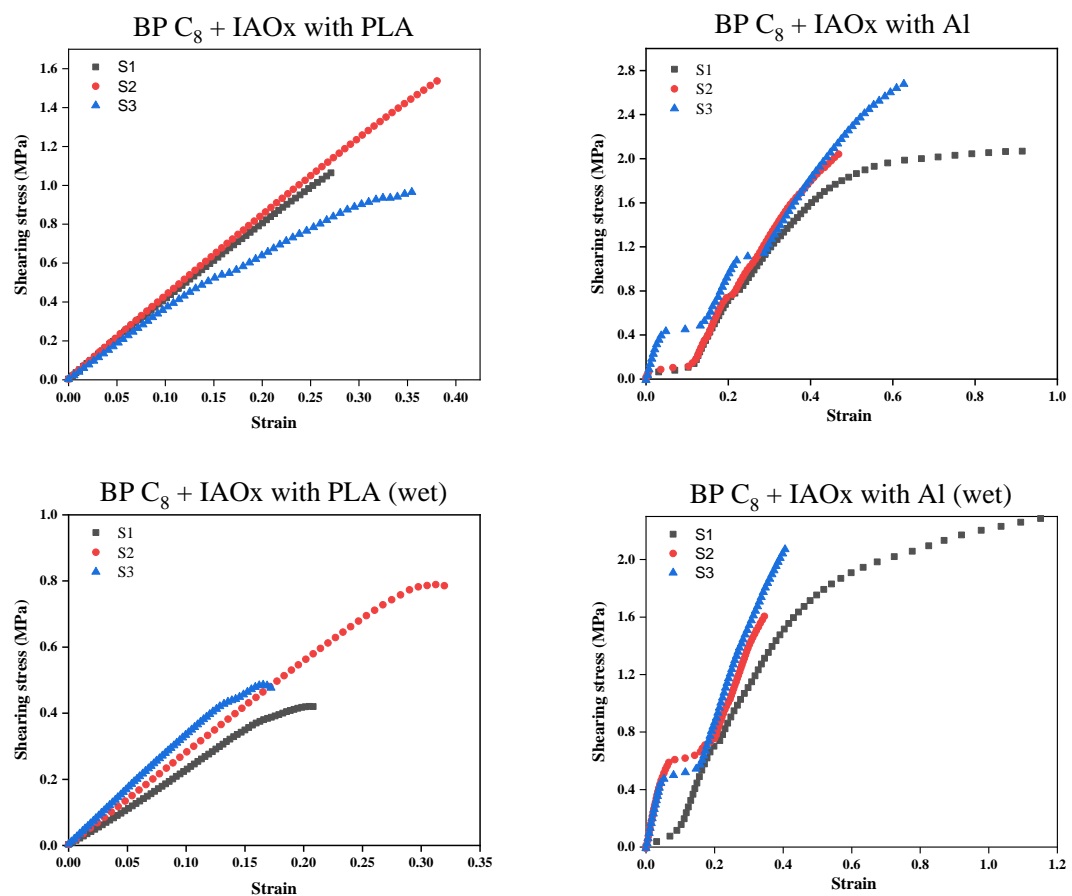

Figure 3.10. Shearing stress versus strain data generated during the lap shear tests on Left) PLA and right) Alumina under ambient conditions and bottom) after immersion in water.

Nevertheless, these findings indicate that the $\mathrm{BP}_{-} \mathrm{C}_{8}$ based polymers developed in this study have potential as adhesive, not only for alumina, but also for hydrogen bond accepting thermoplastic plastic polymers, such as polylactide. Note, though we have observed an excellent adhesion of the developed polymers to glass, we have not identified its performance due to safety considerations. 


\subsubsection{Employing hydrolysis to control thermoset properties and recover the $\mathrm{BP}-\mathrm{C}_{8}$ monomer}

From the findings reported above, it is evident that water plays a crucial role on the chemical stability and on both the thermal and mechanical response of the BP$\mathrm{C}_{8}$ based thermosets. When these thermosets are exposed for sufficiently long time to moisture, hydrolysis continues until all ester bonds are broken. As can be deduced from Scheme 3.1, only the carboxylic acid moieties of $\mathrm{BP}-\mathrm{C}_{8}$ are transformed into esters upon polymerization, hence hydrolysis of the ester bonds would liberate the original carboxylic acids groups and thus result in the release of the $\mathrm{BP}-\mathrm{C}_{8}$ monomer. As explained earlier, the use of an excess of IAox facilitates the generation of tertiary amide bonds ( $k_{2}$ reaction Scheme 3.1), and thus determines the cross-linking density. As a result, in addition to the liberation of $\mathrm{BP}-\mathrm{C}_{8}$, a combination of N,N'-bis(2hydroxyethyl)benzene-1,3-dicarboxamide (IAda) (Scheme 3.3, left) and the hydrolysis products containing branched / cross-linked species connected through tertiary amide bonds (Scheme 3.3, right) are liberated depending on the cross-linking density of the thermoset prior to hydrolysis. Though ester bond hydrolysis suppresses the mechanical properties, it also provides an opportunity to chemically recycle the liberated components. In a broad generality, two scenarios can be envisioned for the hydrolysis: Scenario 1, where the hydrolysis of the thermosets generates strictly BP$\mathrm{C}_{8}$ and IAda components and scenario 2, where the $\mathrm{BP}-\mathrm{C}_{8}$ is selectively liberated after hydrolysis. Scenario 1 is likely to occur when the branching reaction $\left(k_{2}\right.$ reaction in Scheme 3.1) causing crosslinking can be suppressed, i.e. in polymer 1, having no excess of IAox and no cross-linking catalyst (TPP). In contrast, the second scenario is more likely to occur in the samples having extensive branching and cross-linking, for example in polymer 5; On using an excess of IAox, thereby promoting crosslinking, all the IA-da moieties during hydrolysis are prevented from breaking off the polymer chain as they are connected through tertiary amide bonds.

Experiments have been performed to evaluate the feasibility of both scenarios. With respect to the first scenario, we attempted to hydrolyze polymer 1 . The experiment is performed by placing the polymer at atmospheric pressure in a pressure vessel filled with demineralized water. Next, the pressure vessel is closed and heated to $130{ }^{\circ} \mathrm{C}$ for at least three hours while water is maintained in its superheated state. After cooling, the polymer is found to be almost fully hydrolyzed, concluded by the fact that a clear solution is obtained combined with the fact that demineralized water is not a solvent for the original polymer. Since the $\mathrm{BP}-\mathrm{C}_{8}$ monomer could be recrystallized from a water/methanol mixture, methanol was added to the supernatant and the ensuing mixture was stored at $5{ }^{\circ} \mathrm{C}$. Like the purification method for $\mathrm{BP}-\mathrm{C}_{8}$, crystallization of $\mathrm{BP}-\mathrm{C}_{8}$ is observed over time. The 
crystals are filtered and dried after which the presence of $\mathrm{BP}-\mathrm{C}_{8}$ is confirmed using NMR spectroscopy.

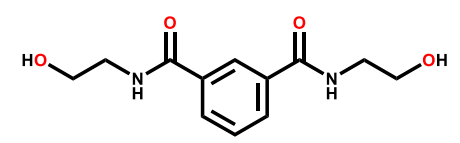

$N^{1}, N^{3}$-bis(2-hydroxyethyl)benzene-1,3-dicarboxamide

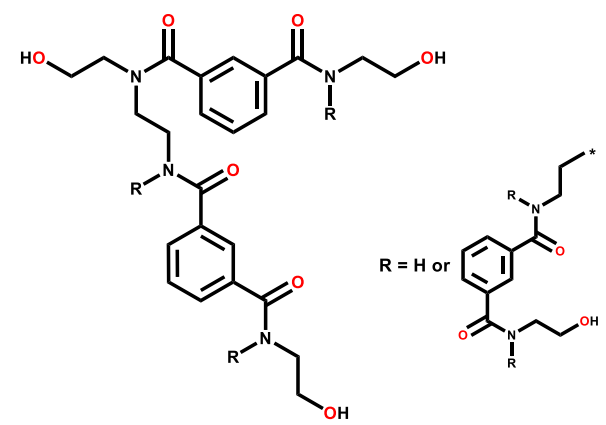

Scheme 3.3. Chemical structures of the expected products after hydrolysis of the polymers used in this study. Left) N,N-bis(2-hydroxyethyl)benzene-1,3dicarboxamide (IAda), which is liberated when the IAox only participated in the $k_{l}$ reaction shown in Scheme 3.1. Right) Possible hydrolysis structures formed after hydrolysis for IAox moieties which did participate in the $k_{2}$ reaction shown in Scheme 3.1. Note, the ratio between the left) and right) structures is dependent on the crosslinking density of the polymer, and hence on the excess of IAox used for polymerization.

Figure 3.11 shows the ${ }^{1} \mathrm{H}-\mathrm{NMR}$ spectra of polymer $\mathbf{1}$ directly after synthesis (blue), polymer 1 after hydrolysis (green), the crystallites obtained after hydrolysis and consecutive recrystallization (red), and the virgin BP- $\mathrm{C}_{8}$ crystals as obtained after synthesis and purification (black). Note, the polymer was hydrolyzed in demineralized water until a fully soluble solution was obtained, after which the water was removed via rotary evaporation and drying in vacuo prior to NMR analysis of the hydrolyzed polymer 1 .

In Figure 3.11, polymer 1 displays the characteristic signals corresponding to both the $\mathrm{BP}_{-} \mathrm{C}_{8}$ and IAda moieties present in the polymer backbone. Additionally, the presence of ester bonds can clearly be observed around $4.2 \mathrm{ppm}$ (resonance 6), corresponding to the methylene protons next to the ester bond. Indeed, after hydrolysis, this proton signal decreases significantly and shifts to $3.5 \mathrm{ppm}$ (resonance 6'), corresponding to the protons next to the free alcohol groups of IAda ${ }^{55}$. Similarly, the methylene protons next to the amide group (resonance 5) shift to $3.3 \mathrm{ppm}$ after hydrolysis (resonance $\left.\mathbf{5}^{\prime}\right)^{55}$, overlapping with resonances $\mathbf{C}$ and $\mathbf{D}$ of the $\mathrm{BP}_{-} \mathrm{C}_{8}$ moiety. The crystals formed from the solution of hydrolyzed polymer $\mathbf{1}$ are predominantly the $\mathrm{BP}_{-} \mathrm{C}_{8}$ monomer $(86 \%)$ whereas the residual $14 \%$ is the IAda compound, as is evident from the red ${ }^{1} \mathrm{H}-\mathrm{NMR}$ spectrum. Although the method to recover the $\mathrm{BP}_{-} \mathrm{C}_{8}$ monomer after polymer hydrolysis is far from being optimized (i.e. 
the $\mathrm{BP}-\mathrm{C}_{8}$ yield after recrystallization is slightly exceeding $40 \%$ ), we consider it a promising route for separation of the used components for further use. Indeed, changing $\mathrm{BP}_{-} \mathrm{C}_{8}$ with $\mathrm{BP}-\mathrm{C}_{2}$, i.e. the dicarboxylic acid obtained after reaction of 1,2-ethanediamine with two itaconic acid molecules, allowed for the retrieval of already $84 \%$ of the $\mathrm{BP}-\mathrm{C}_{2}$ used for polymerization. To be more precise, the crystals were obtained in $95 \%$ yield (of the employed $\mathrm{BP}_{-} \mathrm{C}_{2}$ ) and displayed a purity of $88 \%$ BP- $\mathrm{C}_{2}$ according to NMR analysis (data provided in Appendix B). Please note, the protocol used for the synthesis and hydrolysis is like that reported above.

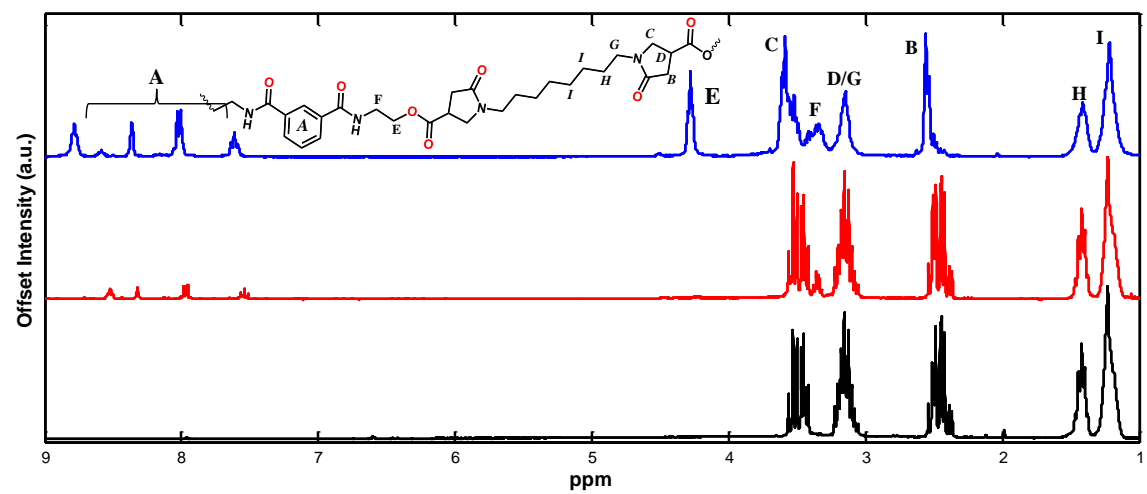

Figure 3.11. ${ }^{1} H$-NMR spectra of, from top to bottom, polymer 1 directly after synthesis (blue), polymer 1 after hydrolysis (green), the crystallites obtained after hydrolysis (red), and the pure BP-C $C_{8}$ monomer as obtained after synthesis and purification (bottom, black).

To address the possibility of the second hydrolysis scenario, we have placed polymer $\mathbf{5}$ for hydrolysis. The sample is cut into bars after exposure to air. The bars are placed in a pressure vessel at $130{ }^{\circ} \mathrm{C}$ together with demineralized water for six hours. In contrast to previous observations, the thermoset remained structurally intact even after subjecting them to such harsh hydrolysis conditions. Apparently, the use of the excess of IAox resulted in enough tertiary amide cross-links (Scheme 3.1) to maintain the cross-linked nature of the thermoset after hydrolysis of the ester bonds. In accordance with our observation in the scenario 1, cooling of the supernatant resulted in the formation of small crystallites that are identified as the $\mathrm{BP}-\mathrm{C}_{8}$ monomer. Both the original thermosets and the hydrolyzed thermosets are analyzed using FTIR after drying (Figure 3.12). In Figure 3.12, the FTIR spectra are normalized on vibration $\mathbf{I}$, corresponding to the vibrations of the phenyl ring $\left(815 \mathrm{~cm}^{-}\right.$ ${ }^{1}$ ). To recall, the absence of the vibrations II (2-oxazoline ring vibrations between 900 and $1000 \mathrm{~cm}^{-1}$ ) suggests a high 2-oxazoline conversion. Additionally, it is evident 
that hydrolysis resulted in the breakage of a large fraction of ester bonds, indicated by decrease in both the $\mathrm{C}-\mathrm{O}$ stretch vibration of the ester bond around $1170 \mathrm{~cm}^{-1}$ (vibration IV) and the $\mathrm{C}=\mathrm{O}$ ester stretch around $1730 \mathrm{~cm}^{-1}$ (vibration VII). Additionally, the decrease in the ester bonds results in an increase in the vibration $\mathbf{V}$ $\left(\mathrm{C}=\mathrm{O}\right.$ stretch of the tertiary amide, $\left.1636 \mathrm{~cm}^{-1}\right)$, and a slight decrease in vibration VI $\left(\mathrm{C}=\mathrm{O}\right.$ stretch of the secondary amide, $\left.1660 \mathrm{~cm}^{-1}\right)$. The removal of the $\mathrm{BP}-\mathrm{C}_{8}$ monomer concentrates the leftover tertiary amide groups, explaining the increase in signal $\mathbf{V}$ after hydrolysis. Furthermore, the decrease in signal VI suggests that the immersion of the thermosets in demineralized water at $130{ }^{\circ} \mathrm{C}$ in a closed pressure vessel also facilitates partial hydrolysis of the secondary amide bonds. Retrospectively, it is possible that vibration VII results from the $\mathrm{C}=\mathrm{O}$ stretch of free carboxylic acid groups of isophthalic acid. Nevertheless, from the FTIR analysis we can confirm that we have developed a procedure to hydrolyze the ester bonds in the thermoset while leaving the tertiary amide bonds intact.

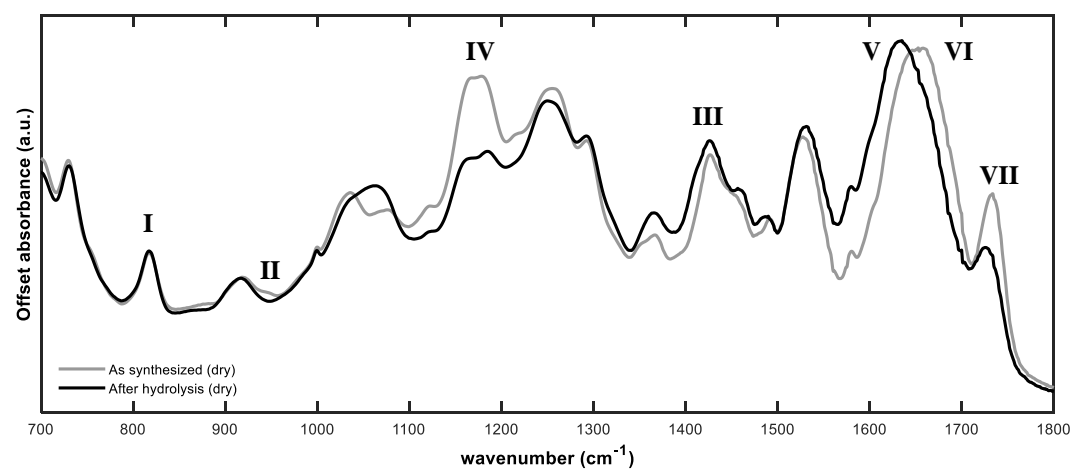

Figure 3.12. FTIR spectra between $800-1800 \mathrm{~cm}^{-1}$ of polymer 5, obtained directly after synthesis (grey) and after hydrolysis for 6 hours, followed by drying in vacuo (black). Note, the FTIR spectra were normalized on the aromatic ring vibration at $815 \mathrm{~cm}^{-1}$.

With respect to the thermo-mechanical behavior, DMTA analysis is performed (after both drying and exposure to ambient conditions and the results are provided in Figure 3.13. From Figure 3.13 left, it is evident that the as-cured polymer 5 exhibits a storage modulus (E') around $3 \mathrm{GPa}$ below its glass transition temperature of $102{ }^{\circ} \mathrm{C}$. Note, the glass transition temperature is identified as the peak in loss modulus (E"). On exposing the same thermoset to roughly $50 \% \mathrm{RH}$ at room temperature, both the storage modulus and $T_{\mathrm{g}}$ decrease to roughly $1 \mathrm{GPa}$ and $93{ }^{\circ} \mathrm{C}$, respectively. This observation is in line with the previously reported results and is attributed to the plasticizing effect of water. 

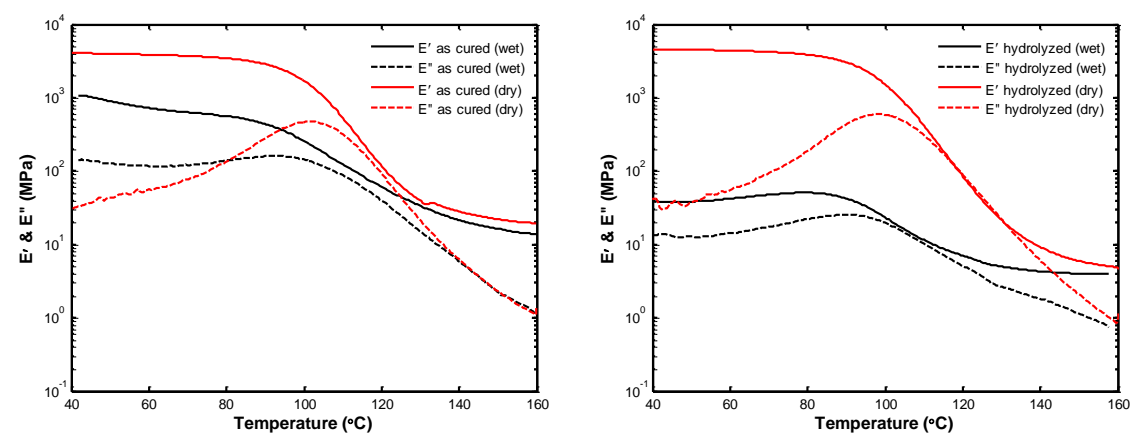

Figure 3.13. DMTA traces showing both $E^{\prime}$ and $E$ " (in MPa) for polymer 5, directly after curing (left) and after hydrolysis in a pressure vessel (right). Please note, both samples were measured in a dried state (red lines) and in a wet state (black line) after exposure to ambient conditions ( $50 \% \mathrm{RH})$.

The hydrolyzed polymer $\mathbf{5}$, in its dry state, exhibits a storage modulus of 3 $\mathrm{GPa}$ and $T_{\mathrm{g}}$ at $98{ }^{\circ} \mathrm{C}$ (Figure 3.13, right). However, on exposing the hydrolyzed polymer to $50 \% \mathrm{RH}$, the material softens considerably resulting in a drop-in storage modulus E' to $40 \mathrm{MPa}$ (below its $T_{\mathrm{g}}$ ) suggesting that the thermoset absorbs significantly more water after hydrolysis compared to the as-synthesized polymer. Interestingly, the $T_{\mathrm{g}}$ of the hydrolyzed sample in the wet state does not display a significant decrease in the presence of water $\left(90{ }^{\circ} \mathrm{C}\right)$. However, from Figure 3.13 right one can observe that the storage modulus for the sample exposed to $50 \% \mathrm{RH}$ gradually increases upon heating, suggesting a continuous evaporation of water during the experiment. Retrospectively, we consider it plausible that the $T_{\mathrm{g}}$ of the material was originally lower than observed but gradually shifts to higher values during the experiment as a result of the continuous evaporation of absorbed water during heating.

The loss of the $\mathrm{BP}-\mathrm{C}_{8}$ monomer in the hydrolyzed sample can be identified by the difference in the storage modulus above the glass transition temperature of the two samples. For comparison, at $160{ }^{\circ} \mathrm{C}$ the as synthesized thermoset exhibits a plateau modulus of $20 \mathrm{MPa}$, whereas the same sample exhibits a plateau modulus of $4 \mathrm{MPa}$ after hydrolysis. Considering the relation $E^{\prime}=3 \rho R T / M_{c}$, where $\rho$ is the density, $\mathrm{T}$ the temperature, $\mathrm{R}$ the ideal gas constant, and $M_{\mathrm{c}}$ the molecular weight between cross-links, and assuming that the sample in its original and hydrolyzed state exhibit a comparable density, we can conclude that $M_{\mathrm{c}}$ of the thermoset after hydrolysis increases by a factor of 5 . The increase in $M_{\mathrm{c}}$ combined with the enhanced absorption of water and softening of the thermoset after hydrolysis is evidence for the breakage of the ester bonds, and at least the partial removal of $\mathrm{BP}-\mathrm{C}_{8}$ monomers. 

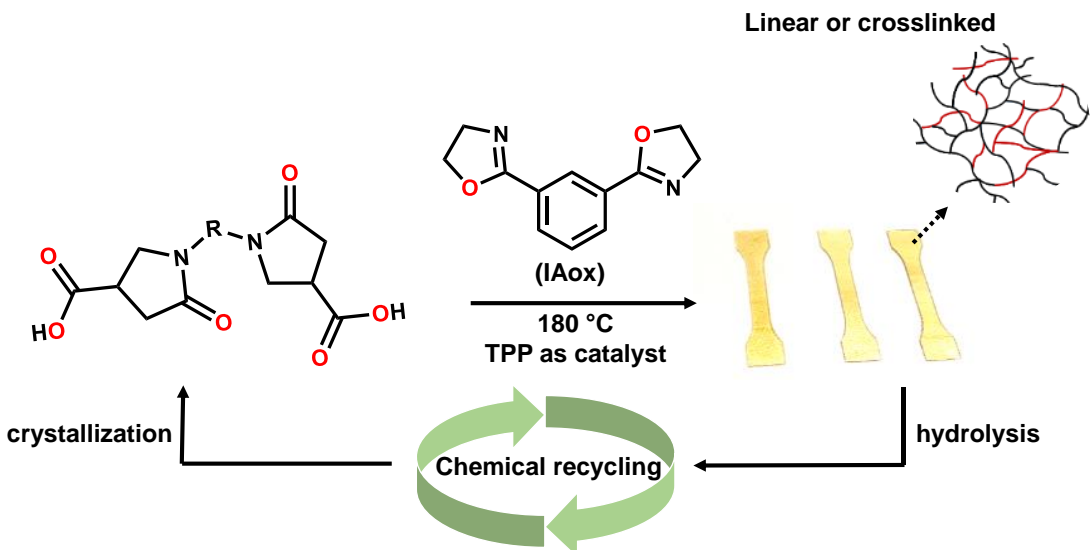

hydrolysis

Scheme 3.4: An overview schematic representation showing the significance of utilizing renewable (bis)pyrrolidone based thermoset resins - with potential towards chemical recycling.

Overall, these findings (summarized in scheme 3.4) demonstrate that this class of thermosets could be promising in applications where structural integrity is desired combined with a slow release of carboxylic acid functionalized molecules, for example in drug delivery applications. The potential of such functional thermosets is currently being explored and will be part of future communications.

\subsection{Conclusions}

In this study we have demonstrated that the cross-link density of poly(esteramide) thermosets based on BP-C 8 and IAox can readily be controlled through the usage of an excess of bis(2-oxazoline) monomer and triphenylphosphite as a catalyst. The synthesized thermosets are brittle and display glass transition temperatures between 60 and $100{ }^{\circ} \mathrm{C}$ directly after curing. Interestingly, all thermosets exhibit a high affinity to moisture. On the absorption of water, the glass transition temperature decreases, and the thermosets display a brittle-to-ductile transition: As anticipated, the mechanical response of the thermoset is found to have a strong dependence on the $T_{\mathrm{g}}$. The presence of water in the thermosets is shown to facilitate slow hydrolysis of the ester bonds, effectively lowering the molecular weight over time. The sensitivity for hydrolysis provides an opportunity for chemical recycling of the BP$\mathrm{C}_{8}$ monomer: On synthesizing non-crosslinked polymers, hydrolysis could be enforced in a pressure vessel, effectively breaking down the polymer to the BP-C 8 monomer and the hydrolyzed structure of IAox, N,N-bis(2-hydroxyethyl)benzene1,3-dicarboxamide. Through preliminary tests, we demonstrate that the BP-C 8 monomer could be crystallized from the ensuing solution providing a potential route 
for its chemical recycling. In addition, when using a thermoset with a high cross-link density, the ester bonds could also be hydrolyzed using the same approach, again liberating the $\mathrm{BP}-\mathrm{C}_{8}$ monomer. However, the tertiary amide cross-links formed through the introduction of an excess of bis(2-oxazoline) proved to maintain the structural integrity of the thermoset even after ester hydrolysis. Overall, these findings demonstrate that this class of thermosets could be promising in applications where structural integrity is desired together with a slow release of carboxylic acid functionalized molecules over time, for example in drug-delivery applications.

For further information, please refer to Appendix B. 


\subsection{References}

(1) Marsh, K.; Bugusu, B. Food Packaging - Roles, Materials, and Environmental Issues: Scientific Status Summary. J. Food Sci. 2007, 72 (3). https://doi.org/10.1111/j.17503841.2007.00301.x.

(2) Gross, R. A.; Kalra, B. Biodegradable Polymers for the Enviroment. Science (80-. ). 2002, 297 (5582), 803-807. https://doi.org/10.1126/science.297.5582.803.

(3) Swift, G. Directions for Environmentally Biodegradable Polymer Research. Acc. Chem. Res. 1993, 26 (3), 105-110. https://doi.org/10.1021/ar00027a005.

(4) Poirier, Y. Green Chemistry Yields a Better Plastic. Nat. Biotechnol. 1999, 17 (10), 960-961. https://doi.org/10.1038/13652.

(5) Winkler, M.; Lacerda, T. M.; Mack, F.; Meier, M. A. R. Renewable Polymers from Itaconic Acid by Polycondensation and Ring-Opening-Metathesis Polymerization. Macromolecules 2015, 48 (5), 1398-1403. https://doi.org/10.1021/acs.macromol.5b00052.

(6) Zhu, Y.; Romain, C.; Williams, C. K. Sustainable Polymers from Renewable Resources. Nature 2016, 540 (7633), 354-362. https://doi.org/10.1038/nature21001.

(7) Ranucci, E.; Liu, Y.; Söderqvist Lindblad, M.; Albertsson, A.-C. New Biodegradable Polymers from Renewable Sources. High Molecular Weight Poly(Ester Carbonate)s from Succinic Acid and 1,3-Propanediol. Macromol. Rapid Commun. 2000, 21 (10), 680-684. https://doi.org/10.1002/1521-3927(20000601)21:10<680::AID-MARC680>3.0.CO;2-Y.

(8) Lindblad, M. S.; Ranucci, E.; Albertsson, A. C. Biodegradable Polymers from Renewable Sources. New Hemicellulose-Based Hydrogels. Macromol. Rapid Commun. 2001, 22 (12), 962-967. https://doi.org/10.1002/1521-3927(20010801)22:12<962::AIDMARC962>3.0.CO;2-E.

(9) Ayadi, F.; Mamzed, S.; Portella, C.; Dole, P. Synthesis of Bis(Pyrrolidone-4-Carboxylic Acid)Based Polyamides Derived from Renewable Itaconic Acid- Application as a Compatibilizer in Biopolymer Blends. Polym. J. 2013, 45 (10), 766-774. https://doi.org/10.1038/pj.2012.206.

(10) Ali, M. A.; Tateyama, S.; Oka, Y.; Kaneko, D.; Okajima, M. K.; Kaneko, T. Syntheses of High-Performance Biopolyamides Derived from Itaconic Acid and Their Environmental Corrosion. Macromolecules 2013, 46 (10), 3719-3725. https://doi.org/10.1021/ma400395b.

(11) Okada, Y.; Banno, T.; Toshima, K.; Matsumura, S. Synthesis and Properties of Polycarboxylate-Type Green Surfactants with S- or N-Linkages. J. Oleo Sci. 2009, 58 (10), 519-528. https://doi.org/10.5650/jos.58.519.

(12) Veličković, J.; Filipović, J.; Djakov, D. P. The Synthesis and Characterization of Poly(Itaconic) Acid. Polym. Bull. 1994. https://doi.org/10.1007/BF00306384.

(13) Willke, T.; Vorlop, K.-D. Biotechnological Production of Itaconic Acid. Appl. Microbiol. Biotechnol. 2001, 56 (3-4), 289-295. https://doi.org/10.1007/s002530100685.

(14) Bressler, E.; Braun, S. Conversion of Citric Acid to Itaconic Acid in a Novel Liquid Membrane Bioreactor. J. Chem. Technol. Biotechnol. 2000, 75 (1), 66-72. https://doi.org/10.1002/(SICI)1097-4660(200001)75:1<66::AID-JCTB176>3.0.CO;2-U. 
(15) Yahiro, K.; Shibata, S.; Jia, S. R.; Park, Y.; Okabe, M. Efficient Itaconic Acid Production from Raw Corn Starch. J. Ferment. Bioeng. 1997, 84 (4), 375-377. https://doi.org/10.1016/S0922338X(97)89265-3.

(16) Tabuchi, T.; Sugisawa, T.; Ishidori, T.; Nakahara, T.; Sugiyama, J. Itaconic Acid Fermentation by a Yeast Belonging to the Genus Candida. Agric. Biol. Chem. 1981, 45 (2), 475-479. https://doi.org/10.1080/00021369.1981.10864534.

(17) Qi, P.; Chen, H.-L. L.; Nguyen, H. T. H.; Lin, C.-C. C.; Miller, S. A. Synthesis of Biorenewable and Water-Degradable Polylactam Esters from Itaconic Acid. Green Chem. 2016, 18 (15), 4170-4175. https://doi.org/10.1039/C6GC01081D.

(18) Wang, Z.; Wei, T.; Xue, X.; He, M.; Xue, J.; Song, M.; Wu, S.; Kang, H.; Zhang, L.; Jia, Q. Synthesis of Fully Bio-Based Polyamides with Tunable Properties by Employing Itaconic Acid. Polym. (United Kingdom) 2014, 55 (19), 4846-4856.

https://doi.org/10.1016/j.polymer.2014.07.034.

(19) Garc??a, J. M.; Garc??a, F. C.; Serna, F.; de la Pe??a, J. L. High-Performance Aromatic Polyamides. Prog. Polym. Sci. 2010, 35 (5), 623-686. https://doi.org/10.1016/j.progpolymsci.2009.09.002.

(20) He, M.; Wang, Z.; Wang, R.; Zhang, L.; Jia, Q. Preparation of Bio-Based Polyamide Elastomer by Using Green Plasticizers. 2016, 86-10. https://doi.org/10.3390/polym8070257.

(21) Roy, M.; Noordzij, G. J. G. J.; Van Den Boomen, Y.; Rastogi, S.; Wilsens, C. H. R. M. R. M. C. H. R. M. Renewable (Bis)Pyrrolidone Based Monomers as Components for Thermally Curable and Enzymatically Depolymerizable 2-Oxazoline Thermoset Resins. ACS Sustain. Chem. Eng. 2018, 6 (4), 5053-5066. https://doi.org/10.1021/acssuschemeng.7b04716.

(22) Farmer, T. J.; Castle, R. L.; Clark, J. H.; Macquarrie, D. J. Synthesis of Unsaturated Polyester Resins from Various Bio-Derived Platform Molecules. Int. J. Mol. Sci. 2015. https://doi.org/10.3390/ijms160714912.

(23) Satoh, K.; Lee, D. H.; Nagai, K.; Kamigaito, M. Precision Synthesis of Bio-Based Acrylic Thermoplastic Elastomer by RAFT Polymerization of Itaconic Acid Derivatives. Macromol. Rapid Commun. 2014. https://doi.org/10.1002/marc.201300638.

(24) Viegas, T. X.; Bentley, M. D.; Harris, J. M.; Fang, Z.; Yoon, K.; Dizman, B.; Weimer, R.; Mero, A.; Pasut, G.; Veronese, F. M. Polyoxazoline: Chemistry, Properties, and Applications in Drug Delivery. Bioconjug. Chem. 2011, 22 (5), 976-986. https://doi.org/10.1021/bc200049d.

(25) Ramiasa, M. N.; Cavallaro, A. A.; Mierczynska, A.; Christo, S. N.; Gleadle, J. M.; Hayball, J. D.; Vasilev, K. Plasma Polymerised Polyoxazoline Thin Films for Biomedical Applications. Chem. Commun. 2015, 51 (20), 4279-4282. https://doi.org/10.1039/c5cc00260e.

(26) De La Rosa, V. R. Poly(2-Oxazoline)s as Materials for Biomedical Applications. J. Mater. Sci. Mater. Med. 2014, 25 (5), 1211-1225. https://doi.org/10.1007/s10856-013-5034-y.

(27) Sedlacek, O.; Monnery, B. D.; Filippov, S. K.; Hoogenboom, R.; Hruby, M. Poly(2Oxazoline)s - Are They More Advantageous for Biomedical Applications than Other Polymers? Macromol. Rapid Commun. 2012, 33 (19), 1648-1662. https://doi.org/10.1002/marc.201200453. 
(28) Hruby, M.; Filippov, S. K.; Panek, J.; Novakova, M.; Mackova, H.; Kucka, J.; Vetvicka, D.; Ulbrich, K. Polyoxazoline Thermoresponsive Micelles as Radionuclide Delivery Systemsa. Macromol. Biosci. 2010, 10 (8), 916-924. https://doi.org/10.1002/mabi.201000034.

(29) Fijten, M. W. M. M.; Haensch, C.; Lankvelt, B. M. V. Van; Hoogenboom, R.; Schubert, U. S. Clickable Poly(2-Oxazoline)s as Versatile Building Blocks. Macromol. Chem. Phys. 2008, 209 (18), 1887-1895. https://doi.org/10.1002/macp.200800226.

(30) Hoogenboom, R.; Schlaad, H. Bioinspired Poly(2-Oxazoline)S. Polymers (Basel). 2011, 3 (4), 467-488. https://doi.org/10.3390/polym3010467.

(31) Lava, K.; Verbraeken, B.; Hoogenboom, R. Poly(2-Oxazoline)s and Click Chemistry: A Versatile Toolbox toward Multi-Functional Polymers. Eur. Polym. J. 2015, 65, 98-111. https://doi.org/10.1016/j.eurpolymj.2015.01.014.

(32) Boerman, M. A.; Van Der Laan, H. L.; Bender, J. C. M. E.; Hoogenboom, R.; Jansen, J. A.; Leeuwenburgh, S. C.; Van Hest, J. C. M. Synthesis of PH- and Thermoresponsive Poly(2-nPropyl-2-Oxazoline) Based Copolymers. J. Polym. Sci. Part A Polym. Chem. 2016, 54 (11), 1573-1582. https://doi.org/10.1002/pola.28011.

(33) Hoogenboom, R. Poly(2-Oxazoline)s: A Polymer Class with Numerous Potential Applications. Angew. Chemie - Int. Ed. 2009, 48 (43), 7978-7994. https://doi.org/10.1002/anie.200901607.

(34) Seo, Y.; Schulz, A.; Han, Y.; He, Z.; Bludau, H.; Wan, X.; Tong, J.; Bronich, T. K.; Sokolsky, M.; Luxenhofer, R.; et al. Poly(2-Oxazoline) Block Copolymer Based Formulations of Taxanes: Effect of Copolymer and Drug Structure, Concentration, and Environmental Factors. Polym. Adv. Technol. 2015, 26 (7), 837-850. https://doi.org/10.1002/pat.3556.

(35) Lorson, T.; Lübtow, M. M.; Wegener, E.; Haider, M. S.; Borova, S.; Nahm, D.; Jordan, R.; Sokolski-Papkov, M.; Kabanov, A. V; Luxenhofer, R. Poly(2-Oxazoline)s Based Biomaterials: A Comprehensive and Critical Update. Biomaterials 2018, 178, 204-280.

https://doi.org/https://doi.org/10.1016/j.biomaterials.2018.05.022.

(36) Hahn, L.; Lübtow, M. M.; Lorson, T.; Schmitt, F.; Appelt-Menzel, A.; Schobert, R.; Luxenhofer, R. Investigating the Influence of Aromatic Moieties on the Formulation of Hydrophobic Natural Products and Drugs in Poly(2-Oxazoline)-Based Amphiphiles. Biomacromolecules 2018, 19 (7), 3119-3128. https://doi.org/10.1021/acs.biomac.8b00708.

(37) Cesana, S.; Kurek, A.; Baur, M. A.; Auernheimer, J.; Nuyken, O. Polymer-Bound Thiol Groups on Poly(2-Oxazoline)S. Macromol. Rapid Commun. 2007, 28 (5), 608-615. https://doi.org/10.1002/marc.200600737.

(38) Cesana, S.; Auernheimer, J.; Jordan, R.; Kessler, H.; Nuyken, O. First Poly(2-Oxazoline)s with Pendant Amino Groups. Macromol. Chem. Phys. 2006, 207 (2), 183-192. https://doi.org/10.1002/macp.200500495.

(39) Hartlieb, M.; Pretzel, D.; Wagner, M.; Hoeppener, S.; Bellstedt, P.; Görlach, M.; Englert, C.; Kempe, K.; Schubert, U. S. Core Cross-Linked Nanogels Based on the Self-Assembly of Double Hydrophilic Poly(2-Oxazoline) Block Copolymers. J. Mater. Chem. B 2015, 3 (9), 1748-1759. https://doi.org/10.1039/C4TB02069C.

(40) Kempe, K.; Hoogenboom, R.; Jaeger, M.; Schubert, U. S. Three-Fold Metal-Free Efficient (“Click”) Reactions onto a Multifunctional Poly(2-Oxazoline) Designer Scaffold. 
Macromolecules 2011, 44 (16), 6424-6432. https://doi.org/10.1021/ma201385k.

(41) Tauhardt, L.; Kempe, K.; Gottschaldt, M.; Schubert, U. S. Poly(2-Oxazoline) Functionalized Surfaces: From Modification to Application. Chem. Soc. Rev. 2013, 42 (20), 7998. https://doi.org/10.1039/c3cs60161g.

(42) Hartlieb, M.; Kempe, K.; Schubert, U. S. Covalently Cross-Linked Poly(2-Oxazoline) Materials for Biomedical Applications-from Hydrogels to Self-Assembled and Templated Structures. J. Mater. Chem. B 2015, 3 (4), 526-538. https://doi.org/10.1039/c4tb01660b.

(43) Frump, J. A. Oxazolines. Their Preparation, Reactions, and Applications. Chem. Rev. 1971, 71 (5), 483-505. https://doi.org/10.1021/cr60273a003.

(44) Kidwai, M.; Mohan, R. Solid Supported Reaction of Substituted 2-Oxazoline with Amines under Microwave Irradiation. J. Chinese Chem. Soc. 2003, 50 (5), 1075-1078. https://doi.org/10.1002/jccs.200300152.

(45) Fazio, M. J. Nucleophilic Ring Opening of 2-Oxazolines with Amines: A Convenient Synthesis for Unsymmetrically Substituted Ethylenediamines. J. Org. Chem. 1984, 49 (25), 4889-4893. https://doi.org/10.1021/jo00199a029.

(46) Wilsens, C. H. R. M.; Wullems, N. J. M.; Gubbels, E.; Yao, Y.; Rastogi, S.; Noordover, B. A. J. Synthesis, Kinetics, and Characterization of Bio-Based Thermosets Obtained through Polymerization of a 2,5-Furandicarboxylic Acid-Based Bis(2-Oxazoline) with Sebacic Acid. Polym. Chem. 2015, 6 (14), 2707-2716. https://doi.org/10.1039/c4py01609b.

(47) Böhme, F.; Clausnitzer, C.; Gruber, F.; Grutke, S.; Huber, T.; Pötschke, P.; Voit, B. Hyperbranched Poly(Ether Amide)s via Nucleophilic Ring Opening Reaction of Oxazolines. High Perform. Polym. 2001, 13 (2). https://doi.org/10.1088/0954-0083/13/2/303.

(48) Huber, T.; Böhme, F.; Komber, H.; Kronek, J.; Luston, J.; Voigt, D.; Voit, B. New Hyperbranched Poly(Ether Amide)s via Nucleophilic Ring Opening of 2-OxazolineContaining Monomers. Macromol. Chem. Phys. 1999, 200 (1), 126-133. https://doi.org/10.1002/(SICI)1521-3935(19990101)200:1<126::AID-MACP126>3.0.CO;2-O.

(49) Jakisch, L.; Bo, F.; Komber, H.; Pompe, G. Synthesis and Thermal Polymerization of Aromatic 2-Oxazolines Containing Carboxylic Groups. 1999, 260, 256-260.

(50) Sano, Y. Polymerization of Bis(2-oxazoline) Compounds with Dicarboxylic Acids. J. Polym. Sci. Part A Polym. Chem. 1989, 27 (8), 2749-2760. https://doi.org/10.1002/pola.1989.080270820.

(51) Thai, W.; Sikkenga, D.; Schroeder, W. Production of Renewable Aromatic Compounds, 2013.

(52) Frost, J. W. Synthesis of Biobased Terephthalic Acids and Isophthalic Acids, 2014.

(53) Fegade, S.; Tande, B.; Kubátová, A.; Seames, W.; Kozliak, E. Novel Two-Step Process for the Production of Renewable Aromatic Hydrocarbons from Triacylglycerides. Ind. Eng. Chem. Res. 2015, 54 (39), 9657-9665. https://doi.org/10.1021/acs.iecr.5b01932.

(54) Jenkins, C. L.; Siebert, H. M.; Wilker, J. J. Integrating Mussel Chemistry into a Bio-Based Polymer to Create Degradable Adhesives. Macromolecules 2017, 50 (2), 561-568. https://doi.org/10.1021/acs.macromol.6b02213. 
(55) Håland, T.; Sydnes, L. K. Formation of N,O-Acetals in the Production of X-Ray Contrast Agents. Org. Process Res. Dev. 2014, 18 (10), 1181-1190.

https://doi.org/10.1021/op500177w. 



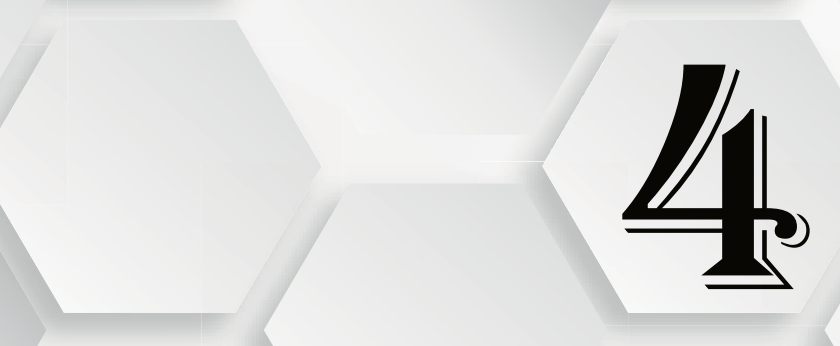

SYNTHESIS OF (FUNCTIONAL) PYRROLIDONE DICARBOXYLIC ACIDS FROM NATURALLY OCCURRING AMINO ACIDS: THEIR POLYMERIZATION, AND VISCOELASTIC BEHAVIOR

This chapter contains unpublished work, submitted to green chemistry journal.

Special thanks to all my students for their hard work and Gijs de Kort for his contribution in the successful completion of this work. 


\title{
In this chapter...
}
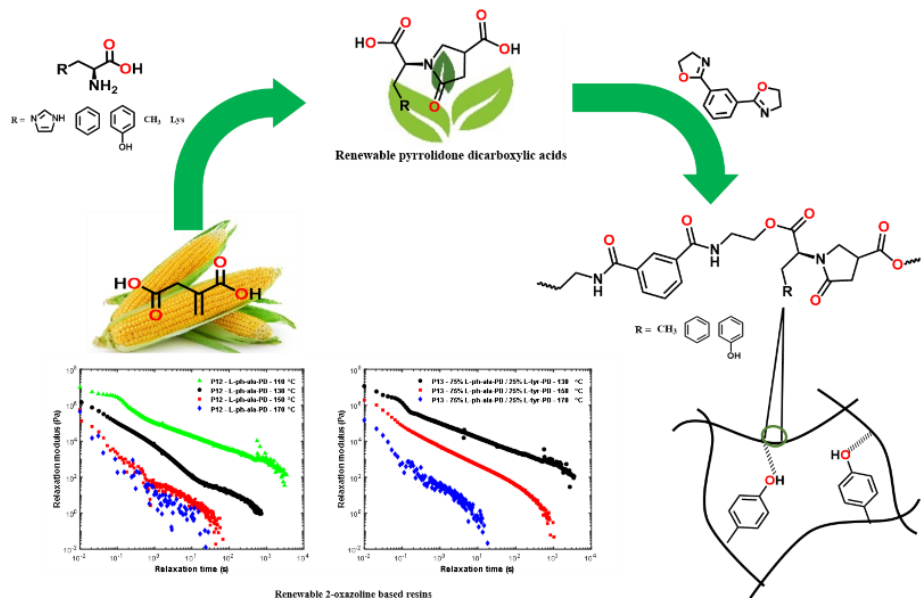

"Synthesis and performance of functional pyrrolidone based dicarboxylic acids from itaconic acid and naturally occurring $L$-amino acids with bis(oxazolines) is evaluated showing secondary interactions in their viscoelastic behavior"

\begin{abstract}
This chapter reports on the synthesis of novel asymmetric functional and renewable biobased carboxylic acids obtained via cascade aza-Michael addition-cyclization reaction using itaconic acid and series of amino acids, including the naturally occurring L-phenyl alanine, L-lysine, L-histidine, and L-tyrosine. The developed monomers prove excellent candidates for thermal polymerization with bis(2oxazolines), yielding thermally stable thermoplastic polymers and thermosets. Furthermore, the presence of non-reactive pendant groups proved to be beneficial in tailoring their relaxation behavior by effectively increasing the secondary interactions.
\end{abstract}




\subsection{Introduction}

Over the years, both academia and chemical industry have focused on the development of materials from renewable resources ${ }^{1,2,3}$, for example from plant oils $^{4,5}$, rosins ${ }^{6}$, terpenes ${ }^{7,8}$ and sugars ${ }^{7,9,10}$. Among the developed chemicals, itaconic acid (IA) has gained considerable attention over the years as its two carboxylic acids and the exo-type double bond make it highly versatile for both chemical modification and polymerization purposes. ${ }^{11,12,13}$ Examples of routes towards itaconic acid based polymers include both chain-growth (i.e. free radical polymerization) ${ }^{14,15,16}$ and stepgrowth (i.e. polycondensation) $)^{17,18}$ techniques. Additionally, itaconic acid, itaconic esters, or itaconic acid based polymers with residual unsaturated bonds can be postfunctionalized via a Michael addition, using for example amines ${ }^{19}$, thiols ${ }^{17,20}$ or acetyl acetone $^{12,21,19,13,22,23}$. When primary amines are used the aza-Michael addition reaction can be followed by a cascade cyclization reaction, leading to $N$-substituted 2-carboxylic acid-pyrrolidone. In particular, the aza-Michael addition-cyclization reaction has proven promising for the generation of monomers for polycondensation, as this reaction allows for the synthesis of (di-)carboxylic acid functionalized pyrrolidone (scheme 4.1, top). As is reported by the groups of Miller, Ali, and Wilsens, these monomers have been used for the development of renewable polyesters ${ }^{25,26,27}$, poly(ester-amide) $\mathrm{s}^{28}$, and polyamides ${ }^{29,27,30,31,30}$ which can be either amorphous or semi-crystalline, enzymatically degradable, bioresorbable, and display potential for chemical recycling. ${ }^{32}$
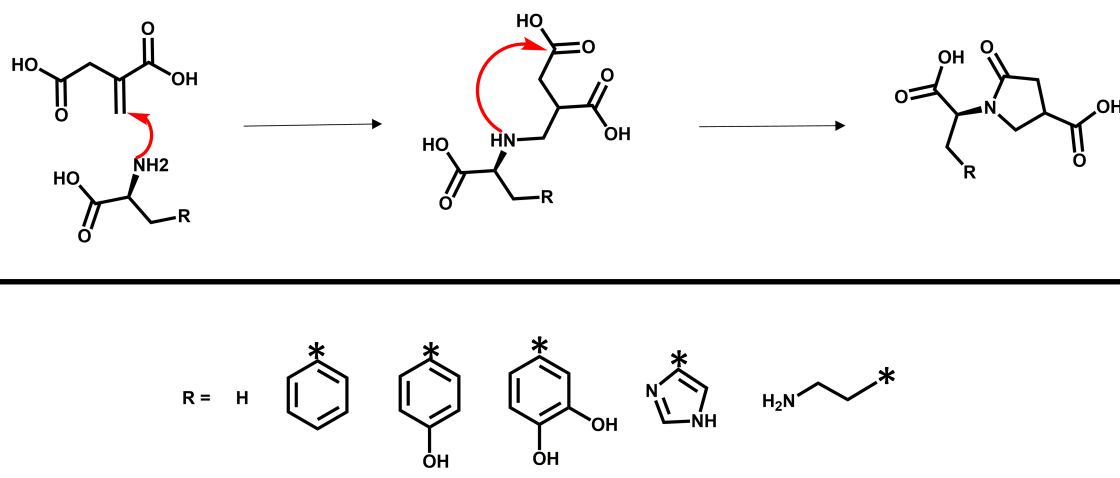

Scheme 4.1. Schematic overview of the aza-Michael addition of itaconic acid with enantiomeric L-amino acids (top, left) and the cascade cyclization with the carboxylic acid at the $\gamma$-position forming a stable 5-membered pyrrolidone ring (top, right). The names and functional groups $(R)$ of the naturally occurring amino acids used in this study are depicted on the bottom of the scheme. 
These pyrrolidone dicarboxylic acids prove particularly interesting for polymerization with bis(2-oxazolines), as the carboxylic acid moiety readily undergoes a ring-opening addition reaction with a 2-oxazoline ring, generating a poly(ester-amide) linkage ( $k_{1}$ reaction, scheme 4.2). In previous work we demonstrated that the basic nature of the pyrrolidone ring drastically enhances the rate of polymerization ${ }^{28}$, effectively providing a platform for the generation of polymers compatible with commercial processing methods (i.e. reactive extrusion or compression molding). To illustrate, the polymerization does not require the removal of a condensate and yields poly(ester-amide)s with molecular weights well above 10 $\mathrm{kg} / \mathrm{mol}$ within one minute of reaction at $180^{\circ} \mathrm{C}$. However, the thermal ring-opening addition polymerization is not limited to the generation of poly(ester-amide)s; 2oxazolines are known to react with aromatic alcohols (yielding ether-amide linkages, $k_{2}$ reaction, scheme 4.2)) and amines (yielding amide-amides linkages) as is reported by the groups of Bohme and Lûston. ${ }^{33,34,35}$ Additionally, 2-oxazolines can react with amides, thereby generating a tertiary amide and a new amide bond ( $k_{3}$ reaction, scheme 4.2). This latter reaction proved an interesting cross-linking mechanism for the generation of thermosets where the cross-linking density can be controlled through the addition of an excess of bis(2-oxazoline $)^{28,36}$.

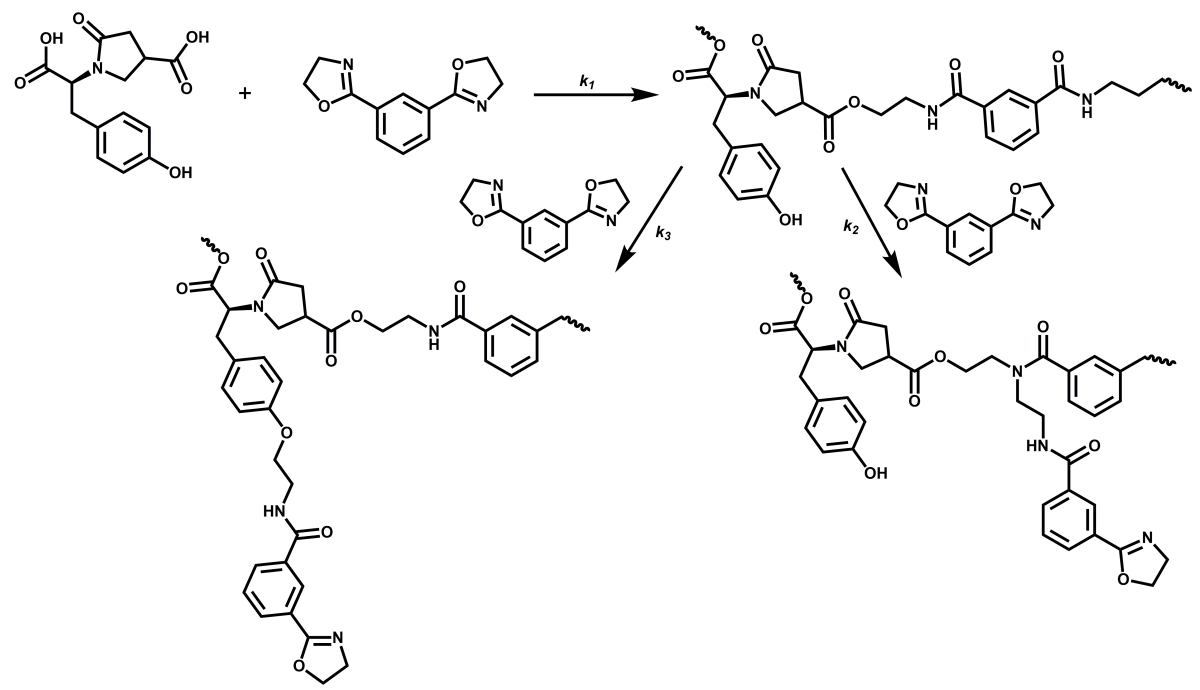

Scheme 4.2. Schematic overview of the thermal ring-opening addition reaction between IAox and the L-tyrosine derived pyrrolidone based dicarboxylic acid (L-tyrPD) yielding poly(ester-amide)s bearing pendant phenyl groups ( $k_{1}$ reaction). Secondly, unreacted 2-oxazoline moieties can react with the aromatic alcohol group of $\mathbf{L}$-tyr-PD ( $k_{2}$ reaction) or amide moiety ( $k_{3}$ reaction) to yield ether-amide or amideamide branches, respectively, both providing mechanisms for cross-linking. 
Though the versatility of the thermal 2-oxazoline curing chemistry may be clear, 2-oxazolines are by far most reactive towards carboxylic acids, likely resulting from their low $\mathrm{p} K_{a}$ and their strong nucleophilic character in the deprotonated state. Accordingly, the difference in reactivity of the 2-oxazoline moiety towards different functional groups (carboxylic acids $>>$ amine $>$ amide $>$ phenol) can potentially be used for the generation of polymers bearing functional side-groups ${ }^{37-40}$. Hence, the purpose of this work is twofold; firstly, we aim to demonstrate that the aza-Michael cascade addition-cyclization reaction can be performed on naturally occurring amino acids to generate renewable dicarboxylic acids having an additional functional group such as a phenol, catechol, amine, or imidazole. To this end, we report on the reaction between itaconic acid and a series of both synthetic and naturally occurring amino acids, including $L$-tyrosine, $L$-DOPA, $L$-lysine, and $L$-histidine (scheme 4.1 ). These starting amino acids are chosen due to the presence of their specific functional groups like phenolic hydroxyl, catechol, tricarboxylic acid or imidazole groups, which might undergo post-polymerization modifications. Additionally, we aim to demonstrate that these monomers are excellent candidates for polymerization with a bis(2-oxazoline) derived from isophthalic acid (IAox), yielding both thermoplastic and thermosetting polymers. Furthermore, we highlight the versatility of the thermal 2-oxazoline curing chemistry by generating poly(ester-amides) bearing pendant phenol groups, for which we observe an increased viscosity and relaxation time, corresponding to the presence of enhanced dynamic network interactions through hydrogen bonding.

\subsection{Experimental section}

\subsubsection{Materials}

Itaconic acid, $L$-tyrosine, $L$-phenyl alanine, $L$-histidine, $L$-alanine, $\beta$-alanine, $L$-lysine, $\gamma$-aminobutyric acid, 11-aminoundecanoic acid, sodium hydroxide, and all used solvents (both normal and deuterated) were purchased from Sigma-Aldrich. 1,3bis (4,5-dihydrooxazol-2-yl)benzene (IAox) was purchased from TCI Europe. The chemicals are used as received, unless it is mentioned otherwise.

\subsubsection{General synthesis procedure of the bis(pyrrolidone) dicarboxylic acids}

The functional pyrrolidone dicarboxylic acids were synthesized in bulk by reacting itaconic acid with the desired amino acid in a $1.2: 1$ molar ratio in the presence of a catalytic amount of water. The slight excess of itaconic acid is required to compensate the side reactions occurring due to isomerization of itaconic acid as reported by Farmer et al. ${ }^{13}$ except in case of L-lysine and L-histidine, double molar ratio of itaconic acid to amino acid was taken to assess reactivity of both amine ( $L$ lysine) and the imidazole ( $L$-histidine) functionalities. Note, sodium hydroxide was 
used to improve the melting/solubility of the amino acid in the reaction mixture. The obtained monomers are abbreviated as $\boldsymbol{L}$-ala-PD (based on $L$-alanine), $\boldsymbol{\beta}$-ala-PD (based on $\beta$-alanine), $\boldsymbol{L}$-lys-PD (based on $L$-lysine), $\boldsymbol{L}$-his-PD (based on $L$-histidine), $\boldsymbol{L}$-tyr-PD (based on $L$-tyrosine), $\boldsymbol{L}$-ph-ala-PD (based on $L$-phenyl alanine), $\boldsymbol{\gamma}$-abaPD (based on $\gamma$-aminobutyric acid) and 11-und-PD (based on 11-aminoundecanoic acid). A detailed overview of the optimized purification conditions used for the various monomers are provided in the table 4.1 shown below.

Table 4.1. Reaction conditions, temperature, time and solvent for purification used for the synthesis of the pyrrolidone dicarboxylic acid monomers synthesized in this study.

\begin{tabular}{|c|c|c|c|c|c|}
\hline Name & Amino acids & $\begin{array}{l}\text { Temper } \\
\text { ature }\end{array}$ & Catalyst & Time & $\begin{array}{c}\text { Solvent for } \\
\text { recrystallization }\end{array}$ \\
\hline$L$-ala-PD & $L$-alanine & $130^{\circ} \mathrm{C}$ & Water & $20 \mathrm{hrs}$. & $\mathrm{MeCN}-\mathrm{EtOAc}$ \\
\hline$\beta$-ala-PD & $\beta$-alanine & $130^{\circ} \mathrm{C}$ & Water & $20 \mathrm{hrs}$. & $\mathrm{MeCN}-\mathrm{EtOAc}$ \\
\hline$\gamma$-aba-PD & $\gamma$ - aminobutyric acid & $130^{\circ} \mathrm{C}$ & Water & 16hrs. & $\mathrm{MeCN}-\mathrm{EtOAc}$ \\
\hline $\begin{array}{l}\text { 11-ada- } \\
\text { PD }\end{array}$ & $\begin{array}{c}\text { 11-aminoundecanoic } \\
\text { acid }\end{array}$ & $110^{\circ} \mathrm{C}$ & Water & $16 \mathrm{hrs}$. & $\mathrm{MeCN}-\mathrm{EtOAc}$ \\
\hline $\begin{array}{c}L \text {-ph-ala- } \\
\text { PD }\end{array}$ & $L$-phenylalanine & $130^{\circ} \mathrm{C}$ & Water & $20 \mathrm{hrs}$. & Acidic water \\
\hline$L$-tyr-PD & $L$-tyrosine & $140^{\circ} \mathrm{C}$ & Water & $48 \mathrm{hrs}$. & $\begin{array}{l}\text { Acidic water - } \\
\text { MeCN }\end{array}$ \\
\hline $\begin{array}{l}L \text {-DOPA- } \\
\text { PD }\end{array}$ & $L$-DOPA & $130^{\circ} \mathrm{C}$ & Water & $20 \mathrm{hrs}$. & $\begin{array}{l}\text { Acidic water - } \\
\quad \mathrm{MeCN}\end{array}$ \\
\hline$L$-lys-PD & $L$-lysine & $130^{\circ} \mathrm{C}$ & Water & $20 \mathrm{hrs}$. & Isopropanol \\
\hline$L$-his-PD & $L$-histidine & $120^{\circ} \mathrm{C}$ & water & $\begin{array}{r}16 \\
\text { hrs. }\end{array}$ & acetone \\
\hline
\end{tabular}

\subsubsection{General melt polymerization procedure}

Polymerization mixtures were prepared on a $3 \mathrm{~g}$ scale by mixing and grinding equimolar amounts of the desired pyrrolidone dicarboxylic acid and 1,3bis(4,5-dihydro-2-oxazolyl) benzene (IAox) in a mortar and pestle. The obtained 
powder mixture was transferred to a $100 \mathrm{~mL}$ round bottom flask fitted with a mechanical stirrer and polymerized at $180{ }^{\circ} \mathrm{C}$ for 30 minutes in inert atmosphere. Whenever required, sampling was performed to monitor the 2-oxazoline conversion over time.

\subsubsection{Characterization methods}

${ }^{1} \mathrm{H}$ NMR and ${ }^{13} \mathrm{C}$ NMR spectroscopy was performed in a Bruker Ultrashield 300 spectrometer (300 MHz magnetic field). Samples were prepared by dissolving $10 \mathrm{mg}$ of monomer or polymer in $0.5 \mathrm{ml}$ deuterated dimethyl sulfoxide (DMSO-d6). All spectra were referenced against tetramethyl silane (TMS).

Molecular weight of the synthesized poly(ester amide)s and dispersities were determined via Gel Permeation Chromatography (GPC) using a PSS SECcurity HPLC system fitted with a Refractive Index detector (RI) running on 1,1,1,3,3,3hexafluoroisopropanol (HFIP) modified with 0,019 \% sodium trifluoroacetate. Separation took place over a PFG combination medium pre-column $(4.6 \times 30 \mathrm{~mm})$ and two PFG combination medium microcolumns all at $7 \mu \mathrm{m}$ particle size (PSS brand, 4.6 x $250 \mathrm{~mm}$, separation range 100-1.000.000 Da). The GPC apparatus was calibrated with poly (methyl methacrylate) standards. The polymers (2-5.0 mg) were dissolved in $1.5 \mathrm{ml}$ of 1,1,1,3,3,3-hexafluoroisopropanol (HFIP) containing 0,019\% sodium trifluoroacetate. After full dissolution, the mixtures were filtered over a 0.2 $\mu \mathrm{m}$ PTFE syringe filter before injection.

Liquid Chromatography - Mass Spectrometry (LC-MS) analysis was performed on a Shimadzu Nexera 2 UHPLC system equipped with a Shimadzu LC-30AD pump, a SPD-M30A photodiode array detector, an ELSD-LTII evaporative light scattering detector and LCMS-2020 single quadrupole detector. The system was run on MilliQ water and LC-MS grade acetonitrile both modified with $0.1 \%$ formic acid. A Waters XSelect CSH C18 column (3.0 with a particle size of 3.5 ųm) was used, operating at $30{ }^{\circ} \mathrm{C}$. The method was setup with a gradient of $5 \%$ acetonitrile in water for $45 \mathrm{~s}$, an increase to $95 \%$ acetonitrile over $2.45 \mathrm{~min}, 30 \mathrm{~s}$ at $95 \%$ followed by flushing back to $5 \%$ acetonitrile for $2 \mathrm{~min}$. Samples were prepared by diluting the reaction mixtures 80 times with methanol or water at $\pm 1 \mathrm{mg} / \mathrm{mL}$. The vial was shaken vigorously prior to filtration over a $0.2 \mu \mathrm{m}$ PFTE filter and consecutive injection. The dilution is performed to ensure that the saturation limit of the detector is not reached. While performing the LC-MS analysis for the quantification of the progress of reaction mixture ELSD is used as the qualitative method, due to the absence of any strong resonance stabilized groups. Due to the nonlinear response of ELSD detectors quantification of reactant and product concentrations is not possible without use of calibration lines. However, in our case, the overlap elution of the different products and reaction intermediates prevented 
quantification of the reaction mixture. Accordingly, we have used LC-MS only for qualitative analysis of the reaction products. All LC-MS peak values are described as $[\mathrm{M}+1]=\mathrm{X}(\mathrm{m} / \mathrm{z})$.

Thermal stability of the synthesized monomers was evaluated using thermogravimetric analysis (TGA). Experiments were performed on a TA Instruments TGA Q500 in a nitrogen rich atmosphere. Samples were heated from 20 to $700{ }^{\circ} \mathrm{C}$, at a heating rate of $10{ }^{\circ} \mathrm{C} / \mathrm{min}$.

The glass transition temperature of the synthesized polymers, all being amorphous, was identified using differential scanning calorimetry (DSC) on a TA Instruments DSC Q2000. Two heating and cooling runs were performed at heating and cooling rates of $10^{\circ} \mathrm{C} / \mathrm{min}$. The glass transition temperature was determined from the second heating run.

The stress relaxation tests and temperature dependency of the viscoelastic response of the developed polymers was measured using an Anton Paar 702 twin drive rheometer. A parallel plate geometry, with a diameter of $12 \mathrm{~mm}$ and gap of 750 $\mu \mathrm{m}$ was used to study the time dependence of the stress relaxation behavior at a strain $(10 \%)$ at various temperatures. A strain of $0.5 \%$ and angular frequency of $1 \mathrm{rad} / \mathrm{s}$ were chosen for the temperature sweep experiments during cooling, starting from 200 ${ }^{\circ} \mathrm{C}$. All tests were performed in the linear viscoelastic regime.

\subsection{Results and discussion}

\subsubsection{Aza-Michael cascade reaction on naturally occurring amino acids}

As is reported in literature, the aza-Michael addition reaction proceeds with addition of an amine as nucleophile to the unsaturated $\mathrm{C}=\mathrm{C}$ bond of itaconic acid or dimethyl itaconate. Recently, Miller and Qi reported on the aza-Michael additioncyclization between itaconic acid and several naturally occurring amino acids bearing hydrocarbon side chains (i.e. $L$-alanine, $L$-valine, $L$-leucine, and $L$-isoleucine, performed in bulk at $130-150{ }^{\circ} \mathrm{C}$ ). The expected pyrrolidone based dicarboxylic acids were isolated after trituration in yields varying from $68-74 \% .{ }^{26}$ However, to the best of our knowledge, the aza-Michael cascade addition-cyclization has not been reported on amino acids having additional functionalities such as phenol (i.e. Ltyrosine or L-DOPA), amine (i.e. L-lysine), or imidazole (i.e. L-histidine) groups. Therefore, to identify its feasibility, we explored the potential of aza-Michael cascade addition-cyclization reaction on a series of amino acids, both naturally occurring and synthetic, for the development of pyrrolidone based dicarboxylic acids (Table 4.2). All monomers are synthesized following the guidelines of green chemistry; the reaction is conducted in bulk for 20-36 hours at temperatures between $120{ }^{\circ} \mathrm{C}$ and 
$140{ }^{\circ} \mathrm{C}$, while using water as catalyst. Next, reduced pressure is applied to the reaction mixtures for one hour to enforce the cascade cyclization reaction and remove the generated water. In turn, purification is performed by crystallization or trituration in solvents such as water and acetonitrile.

Table 4.2. Overview of the various amino acids used for the synthesis of pyrrolidone based dicarboxylic acids, reaction temperatures, isolated yields and thermal stability (graphs provided in Appendix C, Figure C10).

\begin{tabular}{|c|c|c|c|c|}
\hline Name & Amino acid & $\begin{array}{c}\text { Reaction } \\
\text { temperature } \\
\left({ }^{\circ} \mathrm{C}\right)\end{array}$ & $\begin{array}{c}\text { Isolated } \\
\text { Yield } \\
(\%)\end{array}$ & $\begin{array}{l}T_{90}{ }^{\mathrm{B}} \\
\left({ }^{\circ} \mathrm{C}\right)\end{array}$ \\
\hline$L$-ala-PD & L-alanine & 120 & 51 & 265 \\
\hline$\beta$-ala-PD & $\beta$-alanine & 120 & 63 & 222 \\
\hline$\gamma$-aba-PD & $\gamma$-aminobutyric acid & 130 & 98 & 245 \\
\hline 11-und-PD & 11-aminoundecanoic acid & 130 & 93 & 286 \\
\hline$L$-ph-ala-PD & L-phenylalanine & 130 & 69 & 266 \\
\hline$L$-tyr-PD & L-tyrosine & 140 & 65 & 262 \\
\hline$L$-DOPA-PD & L-DOPA & 120 & - & - \\
\hline$L$-lys-PD ${ }^{A}$ & L-lysine & 130 & 17 & 112 \\
\hline$L$-his-PD ${ }^{A}$ & L-histidine & 120 & 51 & 160 \\
\hline
\end{tabular}

${ }^{\mathrm{A}}$ Results depicted of the reaction containing a double molar ratio of itaconic acid, yielding a mixture of both mono and di-substituted pyrrolidone product isolated. ${ }^{\mathrm{B}} \mathrm{T}_{90}$ identifies the temperature where $10 \%$ weight was lost during thermogravimetric analysis performed at a constant heating rate of $10{ }^{\circ} \mathrm{C} / \mathrm{min}$ in a nitrogen atmosphere.

Prior to purification, the reaction mixtures were subjected to LC-MS analysis to identify potential side reactions. Generally, we observe that the reaction proceeds readily under the selected reaction conditions and the desired products are indeed formed for all reactions. As an example, the synthesis of $\boldsymbol{L}$-tyr-PD is described as follows: Itaconic acid (4.3 g, $33 \mathrm{mmol})$ and L-tyrosine (5 g, $27 \mathrm{mmol})$ were added to a $20 \mathrm{~mL}$ microwave glass vial together with sodium hydroxide $(0.54$ $\mathrm{g}, 13.5 \mathrm{mmol})$ and a catalytic amount of water $(13.5 \mu \mathrm{L}$, translating to a concentration of $0.5 \mathrm{ml} / \mathrm{mol}$ amino acid). Next, the mixture was heated to $140^{\circ} \mathrm{C}$ and was allowed to stir for 32 hours in an oil bath to yield a yellow viscous liquid. The product (yield $65 \%$ ) was obtained as white crystals after precipitation and trituration with a mixture 
of dilute acid $(0.1 \mathrm{M} \mathrm{HCl})$ and acetonitrile overnight, followed by filtration and washing with ethyl acetate, and drying in vacuum overnight at $50{ }^{\circ} \mathrm{C}$. ${ }^{1} \mathrm{H}$ NMR $\left.\left(\mathrm{CD}_{3}\right)_{2} \mathrm{~S}=\mathrm{O}, 300 \mathrm{MHz}\right)$ provided as figure 4.2: $\delta 12.80(\mathrm{COOH}, \mathrm{s}, 1 \mathrm{H}), 9,12(\mathrm{OH}, \mathrm{s}$, 1H), 6.99 (d, 2H, J=7.8Hz.), 6.64 (d, 2H, J=8.2Hz.), 4.68 (t, 2H), 3.53 (m, 2H), 3.16 $\left.(\mathrm{m}, 1 \mathrm{H}), 3.05(\mathrm{~m}, 3 \mathrm{H}), 2.86(\mathrm{~m}, 3 \mathrm{H}), 2.36(\mathrm{~m}, 2 \mathrm{H}) .{ }^{13} \mathrm{C} \mathrm{NMR}\left(\mathrm{CD}_{3}\right)_{2} \mathrm{~S}=\mathrm{O}, 300 \mathrm{MHz}\right)$ : $\delta 174.53(\mathrm{C}=\mathrm{OOH}$ ring), $172.0(\mathrm{C}=\mathrm{OOH}), 172.88(\mathrm{NC}=\mathrm{O}$ ring $), 156.28(\mathrm{C}-\mathrm{OH}$ phenyl ring), 129.99 (C-H phenyl ring), 127.80 (C-phenyl ring), 115.59 (C-H phenyl ring), 55.39 ( $\mathrm{N}-\mathrm{CH}$ spacer), 46.30 (N-CH2 ring), 36.13 (N-CH ring), 33.14 (CH2 ring), 33.63 ( $\mathrm{CH} 2$ spacer). Furthermore, the characteristic LC traces of the crude reaction mixture obtained after $\boldsymbol{L}$-tyr-PD synthesis and of the $\boldsymbol{L}$-tyr-PD after purification is provided in figure 4.1 (ELSD signal). The chemical structures of the reaction intermediates, side-products and products are provided in Scheme 4.3.

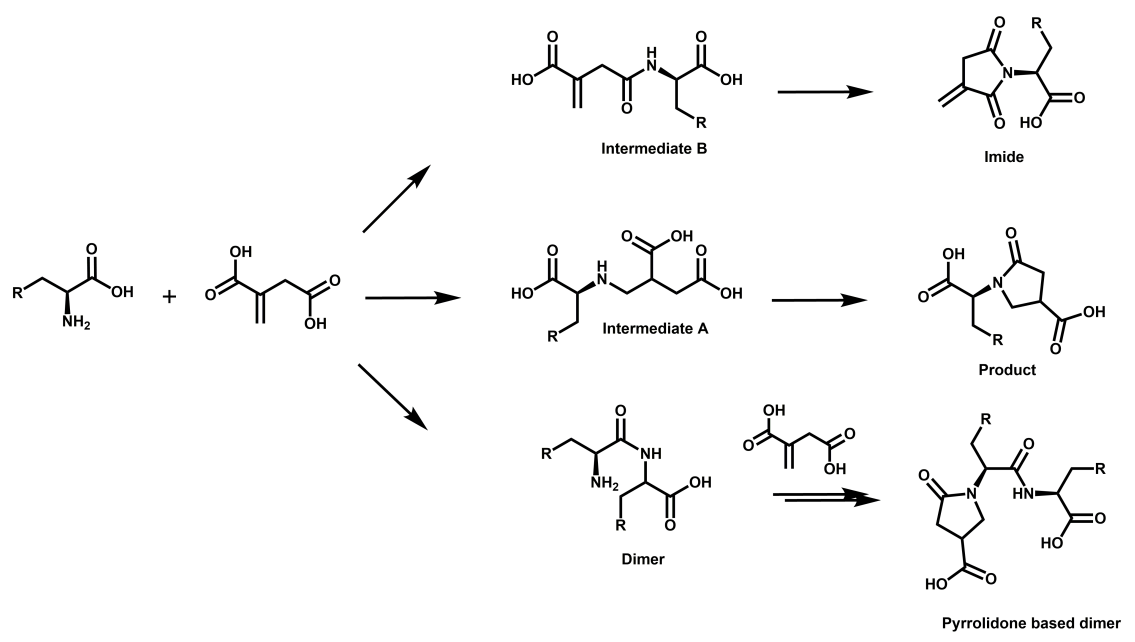

Scheme 4.3. Potential intermediates and products formed during the reaction between itaconic acid and naturally occurring amino acids. Top) amide formation between a carboxylic acid of itaconic acid and the amine of the amino acid, followed by ring closure yielding an imide functionalized carboxylic acid. Middle, desired azaMichael cascade addition-cyclization reaction yielding the pyrrolidone based dicarboxylic acid. Bottom, dimerization between two amino acid molecules, followed by the aza-Michael addition-cyclization cascade reaction, yielding pyrrolidone based dicarboxylic acid dimers.

From the LC trace in Figure 4.1 we observe the presence of two small peaks eluting between 0.25 and 0.4 minutes, which are identified as the residual reactants itaconic acid (IA) and $L$-tyrosine (L-tyr). The desired product $\boldsymbol{L}$-tyr-PD is observed to elute at 2.25 minutes $(\mathrm{m} / \mathrm{z}$ of $294 \mathrm{~g} / \mathrm{mol})$. In addition, we suspect the presence of 
intermediate B (scheme 4.3), corresponding the reaction product where one carboxylic acid of itaconic acid has reacted with the amine of $L$-tyrosine to form an amide with two free dicarboxylic acids, to elute almost simultaneously with the $\boldsymbol{L}$ tyr-PD product.

Identification of intermediate $\mathrm{B}$ in the reaction mixture is motivated by the presence of the two peaks eluting after 2.5 minutes, which are identified as an imide formed after ring closing of Intermediate B while splitting off water (m/z $276 \mathrm{~g} / \mathrm{mol})$, and decarboxylated $\boldsymbol{L}$-tyr-PD product $(\mathrm{m} / \mathrm{z}=249 \mathrm{~g} / \mathrm{mol})$. Please note that purity is not easily determined from LC-MS as 1) the normalized peak area of the different elution peaks does not scale similarly with their respective concentration, 2) the elution peaks of Intermediate B and the $\boldsymbol{L}$-tyr-PD product overlap, and 3) both Intermediate B and $\boldsymbol{L}$-tyr-PD have the same molecular weight.

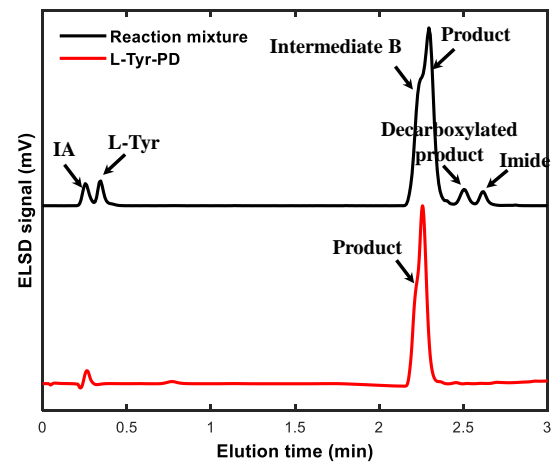

Figure 4.1. Characteristic LC traces of the crude reaction mixture after 32 hours of reaction (top, black line), and the pure $\mathbf{L}-\mathbf{T y}-\boldsymbol{P D}$ product after purification (bottom, red line).

Instead, NMR analysis proved to be more suitable; Purification of the reaction mixture by trituration proved successful as the obtained product was identified solely as $\boldsymbol{L}$-tyr-PD according to ${ }^{1} \mathrm{H}$-NMR analysis (figure 4.2). In Figure 4.2 , the resonances denoted as $e, f$, and $g$ in correspond to the protons of the pyrrolidone ring. Furthermore, the presence of only one resonance at $4.7 \mathrm{ppm}$ (proton $d$ ) is indicative for the purity of the reaction product as this highlights the absence of unreacted L-tyrosine and/or intermediate A (scheme 4.3). Though LC-MS suggests the presence of a trace of intermediate B in the purified $\boldsymbol{L}$-tyr-PD (LC trace, figure 4.1), no resonances are observed between 5 and 6 ppm in NMR analysis, which would be indicative for the presence of unsaturated bonds in either the imide ring or intermediate B. Overall, these findings confirm the high purity of $\boldsymbol{L}$-tyr-PD, given 
that the amounts of intermediate B and imide products are below the detection threshold of the NMR analysis technique.
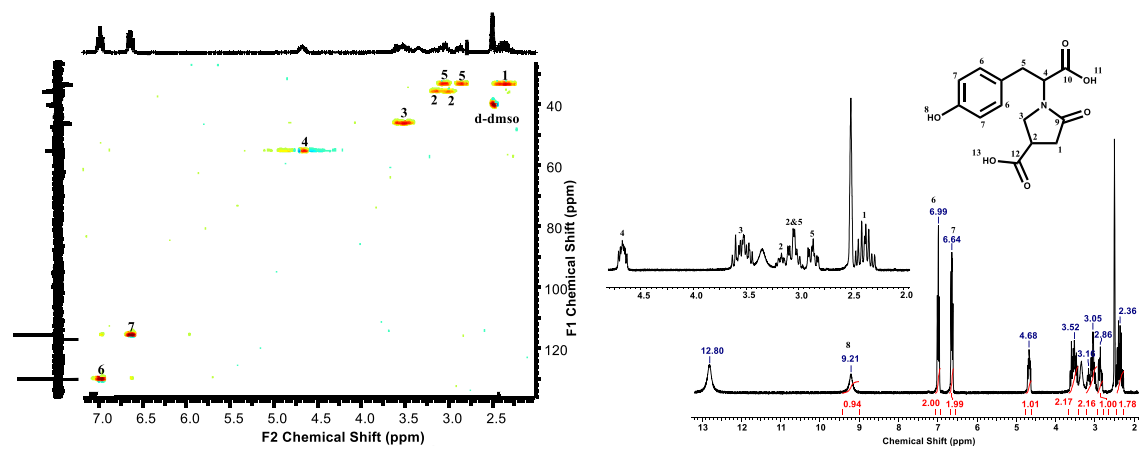

Figure 4.2. HSQC (left) and ${ }^{1} H$ NMR spectra (right) of L-Tyr-PD monomer after purification.

Detailed information on the synthesis and identification of the chemical structure of the monomers synthesized in this study is provided along with the side products formed, and identification of their chemical structures are discussed in detail below. The only exception is for purification and analysis for the L-DOPA-PD monomers, which is due to the challenges arising from their purification and the corresponding complexity of the obtained mixtures.

\subsubsection{Synthesis of 1-(1-carboxy-2-phenylethyl)-5-oxopyrrolidine-3- carboxylic acid (L-ph-ala-PD)}

The synthesis of $\boldsymbol{L}$-ph-ala-PD was achieved in bulk through reaction of itaconic acid (14.2 g, $109.1 \mathrm{mmol})$ and L-phenyl alanine $(15 \mathrm{~g}, 90.7 \mathrm{mmol})$ at $130{ }^{\circ} \mathrm{C}$ for 20 hours using $0.5 \mathrm{ml}$ of water and 0.5 equivalent of sodium hydroxide per mol of $L$-phenyl alanine. Next, residual water in the reaction mixture is removed using reduced pressure, after which a viscous liquid was obtained. The product was obtained by crystallizing from acidic water $(\mathrm{HCl}, 0.1 \mathrm{M})$. The white crystals obtained were dried in vacuo at $80{ }^{\circ} \mathrm{C}$, to yield the product in $69 \%$ yield. Identification of the structure and purity ( $>95 \%$, as no traces of impurities are observed) of the monomer was determined using NMR analysis as is shown in Figure 4.3. ${ }^{1} \mathrm{H}$ NMR $\left.\left(\left(\mathrm{CD}_{3}\right)_{2} \mathrm{~S}=\mathrm{O}\right), 300 \mathrm{MHz}\right): \delta 7.23(\mathrm{~m}, 5.02 \mathrm{H}), 4.71(\mathrm{~m}, 1.0 \mathrm{H}), 3.62(\mathrm{~m}, 2 \mathrm{H}), 3.16(\mathrm{~m}$, $1.2 \mathrm{H}), 3.01(\mathrm{~m}, 2 \mathrm{H})$ and $\left.2.40(\mathrm{~m}, 2 \mathrm{H}) .{ }^{13} \mathrm{C} \mathrm{NMR}\left(\left(\mathrm{CD}_{3}\right)_{2} \mathrm{~S}=\mathrm{O}\right), 300 \mathrm{MHz}\right): \delta 174.49$ $(\mathrm{C}=\mathrm{OOH}$ ring), $172.90(\mathrm{NC}=\mathrm{O}$ ring), $171.85(\mathrm{C}=\mathrm{OOH}), 137.89(\mathrm{C}-\mathrm{H}$ phenyl ring), 128.80 (C-phenyl ring), 126.94 (C-H phenyl ring), 55.18 ( N-CH spacer), 46.43 (N$\mathrm{CH} 2$ ring), 36.09 ( $\mathrm{N}-\mathrm{CH}$ ring), 34.19 ( $\mathrm{CH} 2$ ring), 33.57 ( $\mathrm{CH} 2$ spacer). 

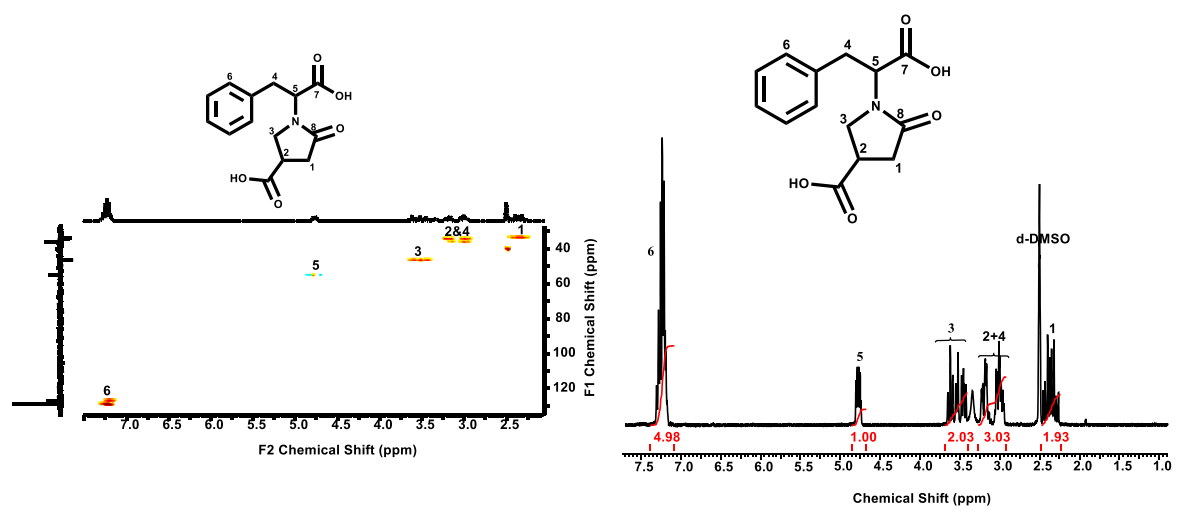

Figure 4.3 HSQC (left) and ${ }^{1} H$ NMR spectra (right) of 1-(1-carboxy-2-phenylethyl)5-oxopyrrolidine-3-carboxylic acid (L-ph-ala-PD).

\subsubsection{Synthesis of 1-(1-carboxyethyl)-5-oxopyrrolidine-3-carboxylic acid (L-ala-PD)}

The synthesis of $\boldsymbol{L}$-ala-PD was achieved in bulk condition through reaction of itaconic acid $(8.8 \mathrm{~g}, 67.6 \mathrm{mmol})$ and $L$-alanine $(5 \mathrm{~g}, 56.1 \mathrm{mmol})$ at $130{ }^{\circ} \mathrm{C}$ for 20 hours using $0.5 \mathrm{ml}$ of water and 0.5 molar equivalent sodium hydroxide mol $L$-alanine used. Next, the residual water in the reaction mixture is removed using reduced pressure, after which a viscous liquid was obtained. The product was obtained by crystallizing from acetonitrile and ethyl acetate. The white crystals obtained were dried in vacuo at $80{ }^{\circ} \mathrm{C}$ to yield the product in $51 \%$ yield. Identification of the structure and purity ( $\geq 95 \%$ as no traces of impurities are observed) of the monomer was determined using NMR analysis as is shown in Figure 4.4. ${ }^{1} \mathrm{H}$ NMR $\left.\left(\left(\mathrm{CD}_{3}\right)_{2} \mathrm{~S}=\mathrm{O}\right), 300 \mathrm{MHz}\right): 4.55(\mathrm{~m}, 1.0 \mathrm{H}), 3.52(\mathrm{~m}, 2 \mathrm{H}), 3.23(\mathrm{~m}, 1 \mathrm{H}), 2.45(\mathrm{~m}, 2$ $\mathrm{H})$, and $1.32(\mathrm{~m}, 3 \mathrm{H}) \cdot{ }^{13} \mathrm{C}$ NMR $\left(\left(\mathrm{CD}_{3}\right)_{2} \mathrm{~S}=\mathrm{O}, 300 \mathrm{MHz}\right): \delta 174.84(\mathrm{C}=\mathrm{OOH}$ ring), 172.93 ( $\mathrm{NC}=\mathrm{O}$ ring), $171.85(\mathrm{C}=\mathrm{OOH}), 49.29(\mathrm{~N}-\mathrm{CH}$ spacer), $45.92(\mathrm{~N}-\mathrm{CH} 2$ ring), 33.97 ( $\mathrm{N}-\mathrm{CH}$ ring), 36.03 ( $\mathrm{CH} 2$ ring), 14.93 ( $\mathrm{CH} 3$ ᄀgroup). 


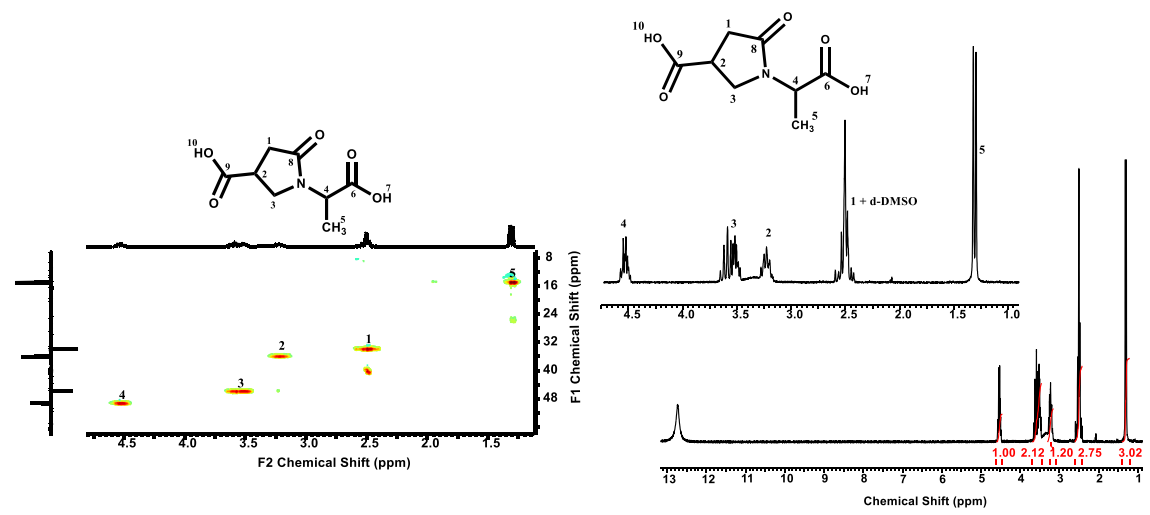

Figure 4.4. HSQC (left) and ${ }^{1} H$ NMR spectra (right) of 1-(1-carboxyethyl)-5oxopyrrolidine-3-carboxylic acid (L-ala-PD)

\subsubsection{Synthesis of 1-(2-carboxyethyl)-5-oxopyrrolidine-3-carboxylic acid ( $\beta$-ala-PD)}

The synthesis of $\boldsymbol{\beta}$-ala-PD monomer was achieved in bulk through reaction of itaconic acid $(8.76 \mathrm{~g}, 67.3 \mathrm{mmol})$ and $\beta$-alanine $(5 \mathrm{~g}, 56.1 \mathrm{mmol})$ at $130{ }^{\circ} \mathrm{C}$ for 20 hours using $0.5 \mathrm{ml}$ of water and 0.5 molar equivalent sodium hydroxide per mol $\beta$ alanine used. The residual water in the reaction mixture is removed using reduced pressure, after which a viscous liquid was obtained. The product was obtained by crystallizing from acetonitrile and ethyl acetate. The white crystals obtained via were dried in vacuo at $80{ }^{\circ} \mathrm{C}$ to obtain the product in $63 \%$ yield. Identification of the structure and purity ( $\geq 85 \%$ ) of the monomer was determined using NMR analysis as is shown in Figures 4.5. Note traces of solvent impurities remain present in the monomer and is not part of the product structure, as is evident from the HMBC, HSQC and COSY spectrum depicted in Figure 4.5 and 4.6 respectively. ${ }^{1} \mathrm{H}$ NMR $\left.\left(\left(\mathrm{CD}_{3}\right)_{2} \mathrm{~S}=\mathrm{O}\right), 300 \mathrm{MHz}\right): 3.52(\mathrm{~m}, 2 \mathrm{H}), 3.38(\mathrm{~m}, 2 \mathrm{H}), 3.16(\mathrm{~m}, 1 \mathrm{H}), 2.44(\mathrm{~m}, 4 \mathrm{H})$. $\left.{ }^{13} \mathrm{C} \mathrm{NMR}\left(\left(\mathrm{CD}_{3}\right)_{2} \mathrm{~S}=\mathrm{O}\right), 300 \mathrm{MHz}\right): \delta 174.84(\mathrm{C}=\mathrm{OOH}$ ring), $172.93(\mathrm{NC}=\mathrm{O}$ ring), $171.85(\mathrm{C}=\mathrm{OOH}), 49.29(\mathrm{~N}-\mathrm{CH}$ spacer$), 45.92(\mathrm{~N}-\mathrm{CH} 2$ ring), $33.97(\mathrm{~N}-\mathrm{CH}$ ring), 36.03 ( $\mathrm{CH} 2$ ring), 14.93 ( $\mathrm{CH} 3$ group). 


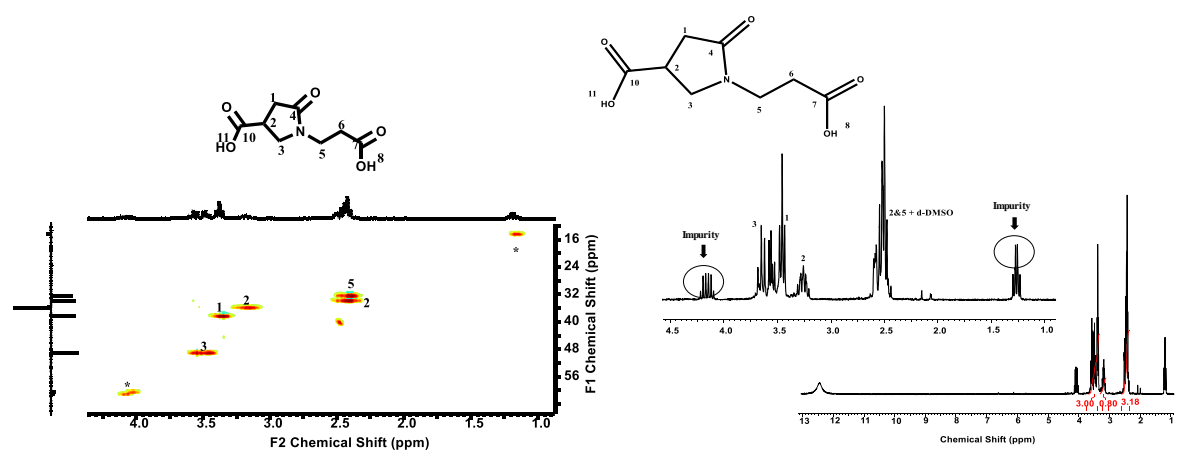

Figure 4.5. HSQC (left) and ${ }^{1} H$ NMR spectra (right) of 1-(2-carboxyethyl)-5oxopyrrolidine-3-carboxylic acid ( $\boldsymbol{\beta}$-ala-PD). NMR spectrum referenced against DMSO signal at 2.5 ppm.
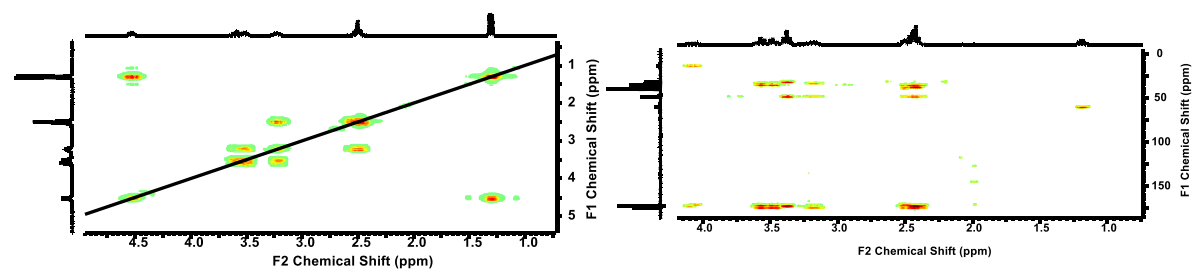

Figure 4.6. $\operatorname{COSY}\left({ }^{1} H-{ }^{1} H\right)$ and $H M B C\left(13 C-{ }^{1} H\right)$ of 1-(1-carboxyethyl)-5oxopyrrolidine-3-carboxylic acid $(\boldsymbol{\beta}$--ala-PD).

\subsubsection{Synthesis of 1-(3-carboxyethyl)-5-oxopyrrolidine-3-carboxylic acid ( $\gamma$-butyric-PD)}

The synthesis of $\boldsymbol{\gamma}$-aba-PD was achieved in bulk through reaction of itaconic acid $(7.57 \mathrm{~g}, 58.2 \mathrm{mmol})$ and $\gamma$-aminobutyric acid $(5 \mathrm{~g}, 48.4 \mathrm{mmol})$ at $130{ }^{\circ} \mathrm{C}$ for 20 hours using $0.5 \mathrm{ml}$ of water and 0.5 molar equivalent sodium hydroxide per mol $\gamma$ aminobutyric acid used. The residual water in the reaction mixture is removed using reduced pressure, after which a viscous liquid was obtained. The product was obtained by crystallizing from acetonitrile and ethyl acetate. The white crystals obtained were dried in vacuo at $80{ }^{\circ} \mathrm{C}$ to obtain the product in $98 \%$ yield. Identification of the structure and purity of the monomer was determined using NMR analysis as is shown in Figures 4.7. The overall purity, is calculated by taking the ratio of the integrals at 5.7 and $6.1 \mathrm{ppm}$, corresponding the two protons next to the unsaturated bond of itaconic acid (0.13) and the integral value of proton 5 from the $\boldsymbol{\gamma}$-aba-PD product (2.0), resulting in a purity of $94 \%$, whereas the remaining $6 \%$ accounts for unreacted itaconic acid. ${ }^{1} \mathrm{H}$ NMR $\left.\left(\left(\mathrm{CD}_{3}\right)_{2} \mathrm{~S}=\mathrm{O}\right), 300 \mathrm{MHz}\right): 3.45$ (m, 2.0 
H), $3.35(\mathrm{~m}, 1.4 \mathrm{H}), 3.18(\mathrm{~m}, 3 \mathrm{H}), 2.46(\mathrm{~m}, 2 \mathrm{H}), 2.17(\mathrm{~m}, 2 \mathrm{H})$, and $1.66(\mathrm{~m}, 2 \mathrm{H})$. ${ }^{13} \mathrm{C}$ NMR $\left.\left(\left(\mathrm{CD}_{3}\right)_{2} \mathrm{~S}=\mathrm{O}\right), 300 \mathrm{MHz}\right): \delta 175.02(\mathrm{C}=\mathrm{OOH}$ ring), $174.44(\mathrm{NC}=\mathrm{O}$ ring), $172.39(\mathrm{C}=\mathrm{OOH}), 48.82(\mathrm{~N}-\mathrm{CH} 2$ ring), $41.39(\mathrm{~N}-\mathrm{CH} 2$ spacer$), 35.89(\mathrm{~N}-\mathrm{CH}$ ring), 31.29 ( $\mathrm{CH} 2$ ring), 22.93 ( $\mathrm{CH} 2$ spacer), 34.11 ( $\mathrm{CH} 2$ spacer).
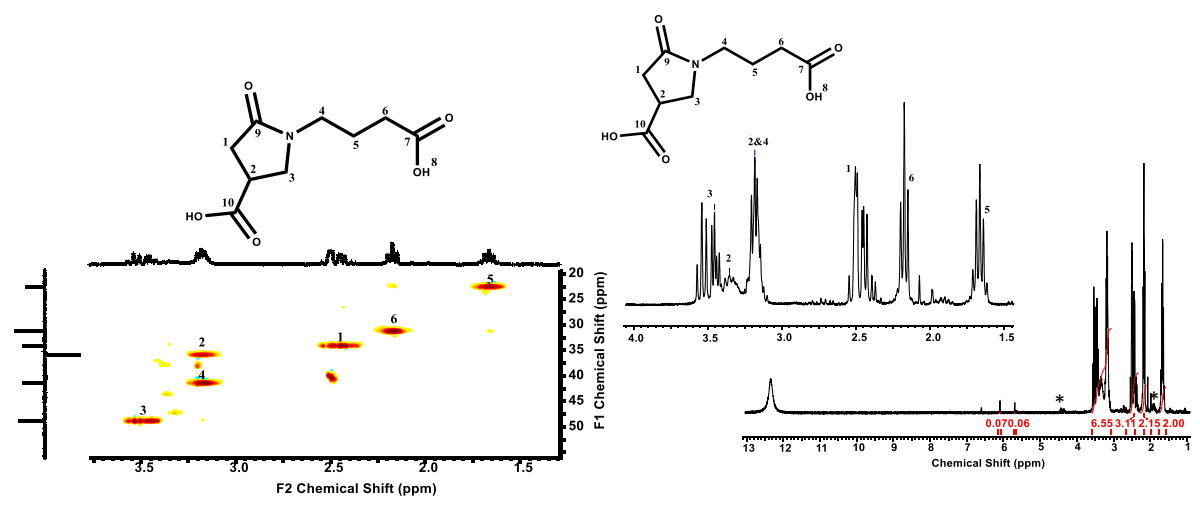

Figure 4.7. HSQC (left) and ${ }^{1} H$ NMR spectra (right) of 1-(3-carboxyethyl)-5oxopyrrolidine-3-carboxylic acid ( $\boldsymbol{\gamma}$-aba-PD).

From Figure 4.7 one can observe the presence of a small trace of itaconic acid, together with the pyrrolidone based dimer and imide as main side-products. Despite the high isolated yield of these monomers, it appears that there are some residual traces of the impurities present in addition to itaconic acid. Retrospectively, the resonances in the NMR spectrum depicted in Figure 4.7 at $1.8 \mathrm{ppm}$ and $2.3 \mathrm{ppm}$ are likely corresponding to residual amounts of pyrrolidone based dimer or its ringclosed product which also aligns with the LC traces of the compound having $\mathrm{m} / \mathrm{z}=$ $283 \mathrm{~g} / \mathrm{mol}$ (shown in figure 4.8).

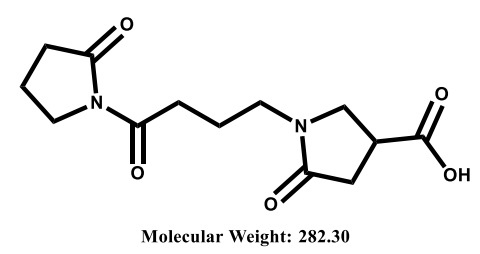

Figure 4.8. Expected chemical structure of the ring-closed pyrrolidone based dimer of $\gamma$-aba-PD.

\subsubsection{Synthesis of 1-(10-carboxyethyl)-5-oxopyrrolidine-3-carboxylic acid (11-und-PD)}

The synthesis of 11-und-PD was achieved in bulk through reaction of itaconic acid (3.88g, $29.8 \mathrm{mmol})$ and 11-aminoundecanoic acid (5g, $24.8 \mathrm{mmol})$ at 
$130{ }^{\circ} \mathrm{C}$ for 20 hours using $0.5 \mathrm{ml}$ of water and 0.5 molar equivalent sodium hydroxide per mol 11-aminoundecanoic acid used. The residual water in the reaction mixture is removed using reduced pressure, after which a viscous liquid was obtained. The product was obtained by crystallizing from acidic water and acetonitrile. The white crystals obtained were dried in oven at $80{ }^{\circ} \mathrm{C}$ to obtain the product in $93 \%$ yield. Identification of the structure and purity $(91 \%)$ of the monomer was determined using NMR analysis as is shown in Figure 4.9. Generally, we observe that there are two signals at 2.0 (integral of 0.2 ) and $2.4 \mathrm{ppm}$ (integral of 0.07), where the signal at 2.0 ppm likely corresponding to the $\mathrm{CH}_{2}$ protons from Intermediate A (aza-Michael adduct). Taking the proportion of this signal and signal $1(2.12 /(0.2+2.12)$, translates into the presence of roughly $9 \mathrm{~mol} \%$ of Intermediate $\mathrm{A} .{ }^{1} \mathrm{H}\left(\left(\mathrm{CD}_{3}\right)_{2} \mathrm{~S}=\mathrm{O}\right)$, $300 \mathrm{MHz}): 3.54$ (m, $2.0 \mathrm{H}), 3.17$ (m, $3 \mathrm{H}), 2.47$ (m, $2 \mathrm{H}), 2.20$ (m, $2 \mathrm{H}), 1.46$ (m, 4 $\mathrm{H})$ and $\left.1.25(\mathrm{~m}, 12 \mathrm{H}) .{ }^{13} \mathrm{C} \mathrm{NMR}\left(\left(\mathrm{CD}_{3}\right)_{2} \mathrm{~S}=\mathrm{O}\right), 300 \mathrm{MHz}\right): \delta 175.05(\mathrm{C}=\mathrm{OOH}$ ring $)$, $174.93(\mathrm{NC}=\mathrm{O}$ ring $), 172.13(\mathrm{C}=\mathrm{OOH}), 48.84(\mathrm{~N}-\mathrm{CH} 2$ ring $), 41.84(\mathrm{~N}-\mathrm{CH} 2$ spacer $)$, $35.86(\mathrm{~N}-\mathrm{CH}$ ring), 34.15 ( $\mathrm{CH} 2$ ring), 24.97 (CH2 spacer), $34.12(\mathrm{CH} 2$ spacer) and 26.59 ( $\mathrm{CH} 2$ spacer).
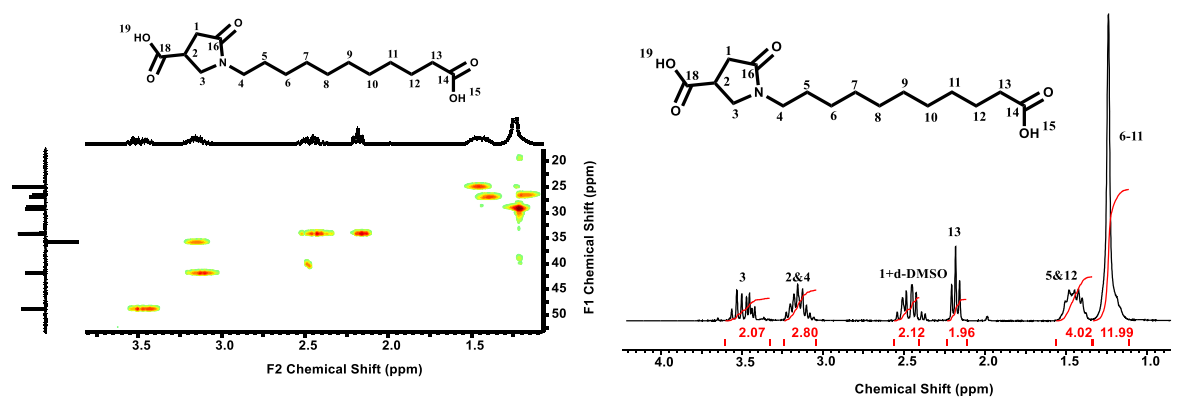

Figure 4.9. . HSQC (left) and ${ }^{1} H$ NMR spectra (right) of 1-(10-carboxyethyl)-5oxopyrrolidine-3-carboxylic acid (11-und-PD).

\subsubsection{Synthesis of 1-1'-(1-carboxypentane-1,5-diyl)bis(5-oxopyrrolidine- 3-carboxylic acid (L-lys-PD)}

The synthesis of $\boldsymbol{L}$-lys-PD monomer was performed in bulk through reaction of itaconic acid $(10.7 \mathrm{~g}, 82.2 \mathrm{mmol})$ and $L$-lysine $(5 \mathrm{~g}, 34.2 \mathrm{mmol})$ at $130{ }^{\circ} \mathrm{C}$ for 20 hours using $0.5 \mathrm{ml}$ of water and 0.5 molar equivalent sodium hydroxide per mol $L$ lysine used. The residual water in the reaction mixture is removed using reduced pressure, after which a viscous liquid was obtained. The product was obtained by precipitation and trituration with acetonitrile. The product was obtained as yellow crystals in a yield of $17 \%$ after drying in vacuo at $80^{\circ} \mathrm{C}$. Identification of the structure 
of the monomer was determined using NMR analysis as is shown in Figure $4.10 .{ }^{1} \mathrm{H}$ NMR (CDCL3 + d-TFA, 300 MHz): 4.85 (m, 1 H), 3.85 (m, $2 \mathrm{H}), 3.51$ (m, $3 \mathrm{H}), 3.45$ $(\mathrm{m}, 4 \mathrm{H}), 3.10(\mathrm{~m}, 4 \mathrm{H}), 1.94+2.16(\mathrm{~m}, 2 \mathrm{H}), 1.72(\mathrm{~m}, 2 \mathrm{H})$ and $1.42(\mathrm{~m}, 2 \mathrm{H}) .{ }^{13} \mathrm{C}$ NMR (CDCL3 + d-TFA, $300 \mathrm{MHz}): \delta 178.05(\mathrm{C}=\mathrm{OOH}$ ring), $175.58(\mathrm{NC}=\mathrm{O}$ ring), $175.63(\mathrm{C}=\mathrm{OOH}), 46.83(\mathrm{~N}-\mathrm{CH} 2$ spacer), $50.12(\mathrm{~N}-\mathrm{CH} 2$ ring), $36.05(\mathrm{~N}-\mathrm{CH}$ ring), 43.15 ( $\mathrm{CH} 2$ ring), 33.61 ( $\mathrm{CH} 2$ spacer), 27.46 ( $\mathrm{CH} 2$ spacer) and 22.75 ( $\mathrm{CH} 2$ spacer).
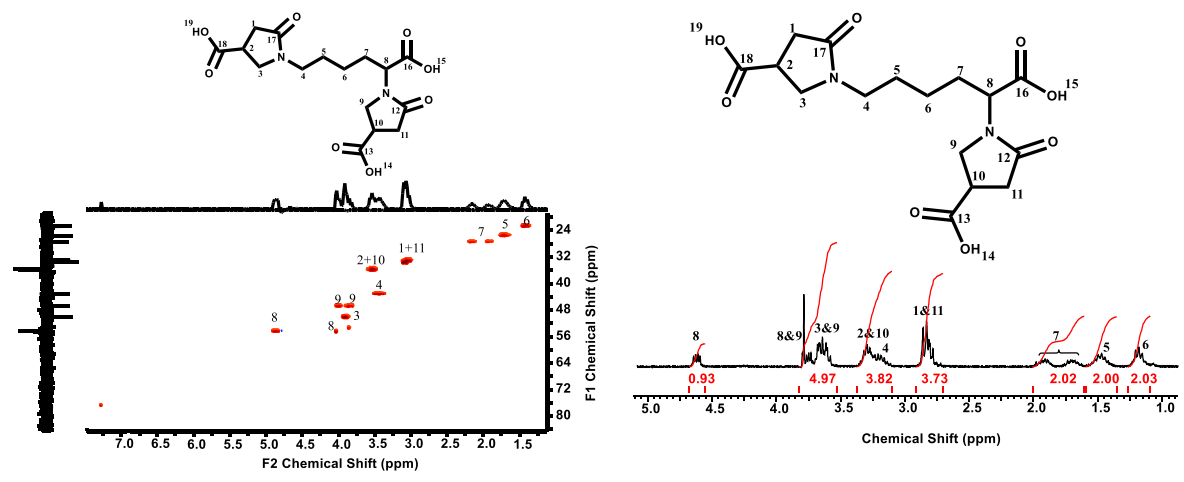

Figure 4.10. HSQC (left) and ${ }^{1} H$ NMR spectra (right) of 1-1'-(1-carboxypentane1,5-diyl)bis(5-oxopyrrolidine-3-carboxylic acid (L-lys-PD).

As is shown in the LC-MS traces of the reaction product of the $\boldsymbol{L}$-lys-PD monomer (Appendix C, Figure C6), we observe the presence of two products; a disubstituted (major) and mono-substituted (minor) component. Considering the integral of the peak of resonance 8 as accounting for one proton (as standard value, Figure 4.10), then the integrals of 5, 6, and 7 account for six protons (corresponding to the L-lysine backbone). In turn, when both amine groups have participated in the aza-Michael addition-cyclization cascade reaction, then we expect two pyrrolidone rings per lysine molecule, hence the integral of the signals 1 and 11 should account for four protons. However, as is observed from Figure 4.10, we only observe the integral to be 3.73 , suggesting that we are dealing with a mixture of both mono- and di-substituted products. Similarly, the integral value of the signals at 3 and 9 corresponding to two $\mathrm{CH}_{2}$ groups of pyrrolidone ring $\left(\mathrm{CH}_{2}-\mathrm{N}\right.$ bond) appears as 3.82 instead of 4.0. This lowered integral value of the signals corresponding to the pyrrolidone ring suggests there is a mixture of both mono- and di-substituted $\boldsymbol{L}$-lysPD. Accordingly, the purity of the obtained $\boldsymbol{L}$-lysine-PD monomer was determined using the formula below, based on the assumption that all molecules are disubstituted; 


$$
\begin{gathered}
\frac{\int(\text { signal } 1+11)_{\text {theoritical }}-\int(\text { signal 1 } 11)_{\text {obtained }}}{4} \times 100 \% \\
=\frac{4-3.73}{4} \times 100 \% \\
=7.5 \%
\end{gathered}
$$

Hence, the product is isolated as $92.5 \%$ disubstituted product (both the aza-Michael adduct and the ring closed structure), while the $\boldsymbol{L}$-lys-PD product accounts for the remainder $7.5 \%$. It appears that the purification protocol used favors the isolation of the disubstituted product, and hence, is likely the reason for the low yield after purification. Nevertheless, these findings clearly demonstrate that also L-lysine can readily be subjected to reaction with itaconic acid, though it seems to be more favorable to target the synthesis of the pyrrolidone based tricarboxylic acid, rather than having one pendant amine group unreacted.

\subsubsection{Synthesis of 1-(1-carboxy-2-(3,4-dihydroxyphenyl)ethyl)-5- oxopyrrolidine-3-carboxylic acid (L-DOPA-PD)}

The synthesis of $\boldsymbol{L}$-DOPA-PD monomer was achieved in bulk through reaction of itaconic acid $(7.8 \mathrm{~g}, 60 \mathrm{mmol})$ and L-dopamine $(9.85 \mathrm{~g}, 50 \mathrm{mmol})$ at 130 ${ }^{\circ} \mathrm{C}$ for 20 hours using $0.5 \mathrm{ml}$ of water and 0.5 molar equivalent sodium hydroxide per mol $L$-DOPA used. The residual water in the reaction mixture is removed using reduced pressure, after which a viscous liquid was obtained and was analyzed without purification (i.e. purification proved unsuccessful). NMR analysis was performed to confirm the presence of the desired $\boldsymbol{L}$-DOPA-PD product as is shown in Figure 4.11. ${ }^{1} \mathrm{H}$ NMR $\left(\mathrm{D}_{2} \mathrm{O}, 300 \mathrm{MHz}\right): \delta 6.57(\mathrm{~m}, 1 \mathrm{H}), 6.51(\mathrm{~m}, 1 \mathrm{H}), 6.38(\mathrm{~m}, 1 \mathrm{H}), 4.60(\mathrm{~m}$, $0.53 \mathrm{H}), 4.49(\mathrm{~m}, 0.49 \mathrm{H}), 3.39+3.24(\mathrm{~m}, 2.24 \mathrm{H}), 2.96(\mathrm{~m}, 2.11 \mathrm{H}), 2.67(\mathrm{~m}, 1.15$ $\mathrm{H})$ and $2.33(\mathrm{~m}, 2 \mathrm{H}) .{ }^{13} \mathrm{C} \mathrm{NMR}\left(\mathrm{D}_{2} \mathrm{O}, 300 \mathrm{MHz}\right): \delta 174.49(\mathrm{C}=\mathrm{OOH}$ ring $), 172.90$ $(\mathrm{NC}=\mathrm{O}$ ring), $171.85(\mathrm{C}=\mathrm{OOH}), 130.59(\mathrm{C}-\mathrm{H}$ phenyl $\mathrm{OH}$ ring $), 120.85(\mathrm{C}-\mathrm{H}$ phenyl ring), 119.89 (C-H phenyl ring), 116.10 (C-H phenyl ring), 55.96 (N-CH spacer), 47.91 ( N-CH2 ring), 35.70 (N-CH ring), 30.84 (CH2 ring), 33.14 (CH2 ᄀspacer). 


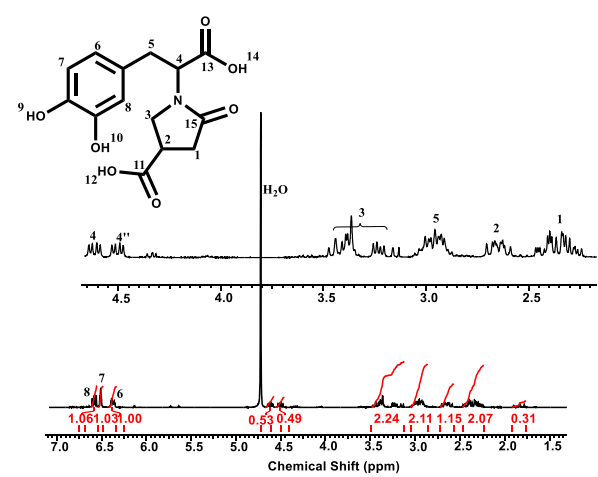

Figure 4.11. ${ }^{1} H$ NMR spectrum of 1-(1-carboxy-2-(3,4-dihydroxyphenyl)ethyl)-5oxopyrrolidine-3-carboxylic acid (L-DOPA-PD).

The ${ }^{1} \mathrm{H}-\mathrm{NMR}$ spectrum in Figure 4.11 shows that the signal 8 corresponds to one aromatic proton $(\delta=6.38 \mathrm{ppm})$. Accordingly, the integral of proton 4 should correspond to a value 1 . However, we observe that these signals (located at $\delta=4.60$ \& $4.49 \mathrm{ppm}$ ) are split located and account for 0.53 and 0.49 respectively. This splitting of signal is expected to correspond to the unreacted $L$-DOPA (preferably in its oxidized form, figure 4.12) and the $\boldsymbol{L}$-DOPA-PD product, respectively. Therefore, this data suggests that roughly, $50 \%$ of the L-DOPA participated in the aza-Michael addition and the remainder of the $L$-DOPA remains present in the unreacted or oxidized state (shown in LC-MS traces, Appendix C, Figure C7).

When evaluating the LC-MS trace of the reaction containing $L$-DOPA, one can observe the presence of both residual $L$-DOPA (denoted as AA) and itaconic acid (provided in Appendix C, Figure C7). The obtained mixture is green, likely resulting from the partial oxidation of $L$-DOPA into quinone and its consecutive cyclization into aminochrome (figure 4.12).

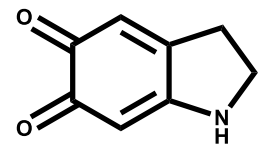

Figure 4.12. Chemical structure of aminochrome resulting from partial oxidation of $L-D O P A$.

Additionally, we observe the elution of peaks having masses of $\mathrm{m} / \mathrm{z}=310$ g/mol ( $\boldsymbol{L}$-DOPA-PD or Intermediate B), m/z = $264 \mathrm{~g} / \mathrm{mol}$ (decarboxylated $\boldsymbol{L}$-DOPAPD or intermediate B) and $\mathrm{m} / \mathrm{z}=248 \mathrm{~g} / \mathrm{mol}$ (reduced decarboxylated form of product of $\boldsymbol{L}$-DOPA-PD). Possible structures of the compounds having a $\mathrm{m} / \mathrm{z}=264 \mathrm{~g} / \mathrm{mol}$ and $248 \mathrm{~g} / \mathrm{mol}$ are provided in Scheme 4.4. As observed from NMR analysis, the $L$-DOPA 
has a poor reactivity as residual $L$-DOPA remains present at the end of the reaction. Combined, the presence of various structures and the poor reactivity explain the challenging purification. As a result, we were unable to obtain the desired product in the pure form and excluded $\boldsymbol{L}$-DOPA-PD from further study.

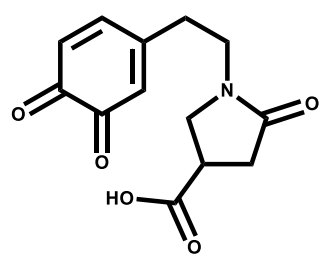

Molecular Weight: $\mathbf{2 6 3 . 2 5}$

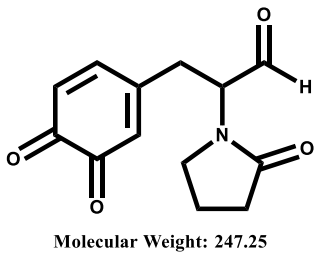

Molecular Weight: $\mathbf{2 4 7 . 2 5}$

Scheme 4.4. Potential chemical structures of the compounds observed in the LC elugram shown in Figure C7 having a $\mathrm{m} / \mathrm{z}=264$ and $248 \mathrm{~g} / \mathrm{mol}$.

\subsubsection{Synthesis of 1-(1-carboxy-2-(1H-imidazol-5-yl) ethyl)-5- oxopyrrolidine-3-carboxylic acid (L-his-PD)}

The synthesis of $\boldsymbol{L}$-his-PD monomer was achieved in bulk through reaction of both equimolar of itaconic acid $(2.5 \mathrm{~g}, 19.3 \mathrm{mmol})$ with $L$-histidine $(3.0 \mathrm{~g}, 19.3$ $\mathrm{mmol})$ and double excess of itaconic acid $(5 \mathrm{~g}, 38.6 \mathrm{mmol})$ with $L$-histidine $(3.0 \mathrm{~g}$, $19.3 \mathrm{mmol}$ ) at $120^{\circ} \mathrm{C}$ for 20 hours using $0.5 \mathrm{ml}$ of water and 0.5 molar equivalent sodium hydroxide per mol $L$-histidine used. The residual water in the reaction mixture is removed using reduced pressure, after which a viscous liquid was obtained. The product was obtained as slightly green crystals by precipitation and trituration with acetone and drying in vacuo at $80{ }^{\circ} \mathrm{C}$. The mono(major) along with traces of disubstituted product was obtained in $43 \%$ yield (Figure 4.13 ), while disubstituted product yielded in $51 \%$ product consisting of both mono and di-substituted product. Purity of both products (equimolar ratio, Figure 4.13 and, double molar ratio of itaconic acid, Figure 4.14) was determined using NMR analysis. ${ }^{1} \mathrm{H}$ NMR (d-D $\mathrm{D}_{2}$, $300 \mathrm{MHz}): \delta 8.80(\mathrm{~m}, 1 \mathrm{H}), 7.26(\mathrm{~m}, 1 \mathrm{H}), 4.37(\mathrm{~m}, 1.29 \mathrm{H}), 3.22(\mathrm{~m}, 4.44 \mathrm{H}), 2.57$ $(\mathrm{m}, 2 \mathrm{H}) .{ }^{13} \mathrm{C}$ NMR $\left(\mathrm{d}-\mathrm{D}_{2} 0,300 \mathrm{MHz}\right): \delta 174.49(\mathrm{C}=\mathrm{OOH}$ ring), $172.90(\mathrm{NC}=\mathrm{O}$ ring), $171.85(\mathrm{C}=\mathrm{OOH}), 130.59(\mathrm{C}-\mathrm{H}$ phenyl $\mathrm{OH}$ ring), 120.85 (C-H phenyl ring), 119.89 (C-H phenyl ring), 116.10 (C-H phenyl ring), $55.96(\mathrm{~N}-\mathrm{CH}$ spacer), $47.91(\mathrm{~N}-\mathrm{CH} 2$ ring), $35.70(\mathrm{~N}-\mathrm{CH}$ ring), $30.84(\mathrm{CH} 2 \mathrm{ring}), 33.14(\mathrm{CH} 2$ spacer $)$. 

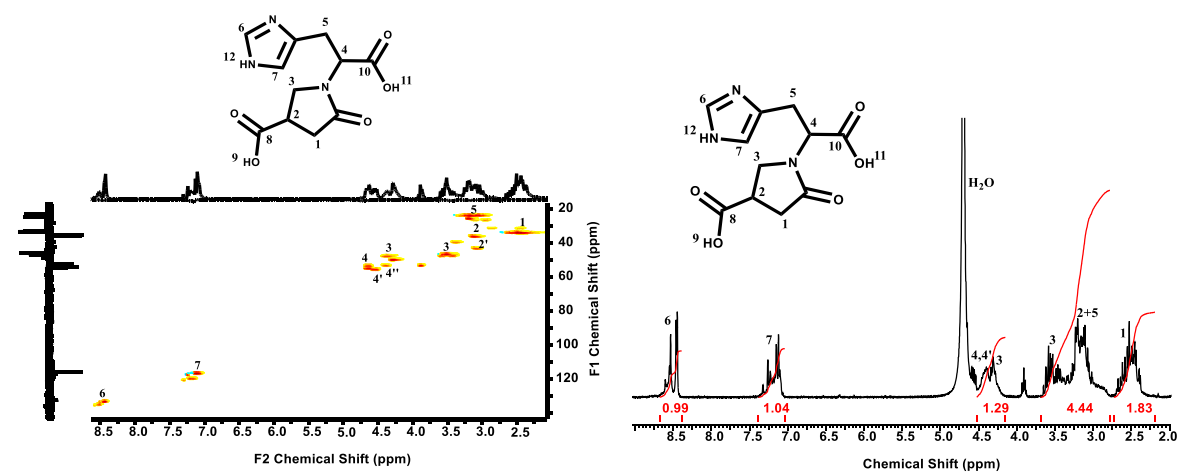

Figure 4.13. HSQC (left) and ${ }^{1} H$ NMR spectra (right) of 1-(1-carboxy-2-(1Himidazol-5-yl)ethyl)-5-oxopyrrolidine-3-carboxylic acid (L-his-PD) containing traces of disubstituted product (L-his-PD), obtained while using equimolar ratio of itaconic acid with L-histidine.

From Figure 4.13 we observe that the signal corresponding to the methylene protons 1 (from the pyrrolidone ring) has an integral value of 1.83 , while the integral of the aromatic protons 6 and 7 of the imidazole ring has a value of 1 . This would imply that there is 0.9 pyrrolidone rings present for every imidazole moiety. In other words, only $90 \%$ of the L-histidine molecules participated in the aza-Michael addition-cyclization cascade reaction and the remainder of the molecules are either unreacted L-histidine or other side-products.

The ${ }^{1} \mathrm{H}$ NMR analysis of the $\boldsymbol{L}$-his-PD product obtained from the reaction mixtures using a 2.1: 1 ratio of itaconic acid and $L$-histidine is provided in Figure 4.14. Using the same method as earlier, we observe that the integrals of imidazole protons 6 and 7 have a value of 1, if the aza-michael addition and ring closure on primary amine happens. However, the integral of the methylene group in the pyrrolidone ring / aza-Michael adduct (signal 1+10) has a value of 3.54 corresponding to $2 \mathrm{H}$ protons, where the signal 1 arises from ring closed pyrrolidone structure, and the remaining 1.54 value corresponds to the $2^{\text {nd }}$ aza-michael addition reaction, which results in 0,77 molar ratio of double aza-michael addition reaction. Hence, it yields $77 \%$ disubstituted products along with traces of unreacted itaconic acid $(<4$ mol \%) (Figure 4.14) as identified by the presence of peaks between 5.5 and $6.5 \mathrm{ppm}$. In summary, the synthesis of $\boldsymbol{L}$-his-PD is only party successful as this compound can participate in a second aza-Michael addition reaction, yielding a multi-functional carboxylic acid. Nevertheless, this monomer mixture might prove promising for the development of renewable thermosets containing imidazole functionality. 

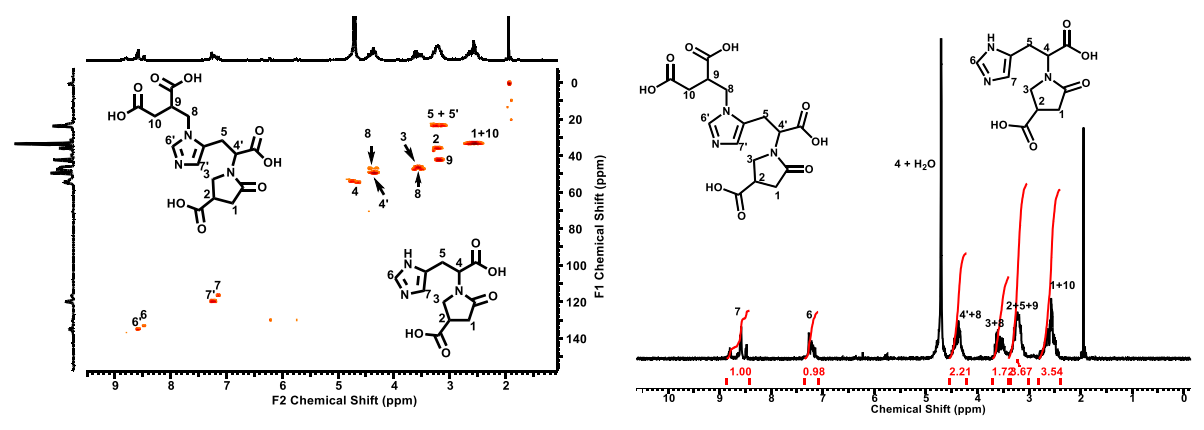

Figure 4.14. HSQC (left) and ${ }^{1} H$ NMR spectra (right) of 1-(1-carboxy-2-(1Himidazol-5-yl)ethyl)-5-oxopyrrolidine-3-carboxylic acid (L-his-PD) obtained while using double excess molar ratio of itaconic acid with L-histidine.
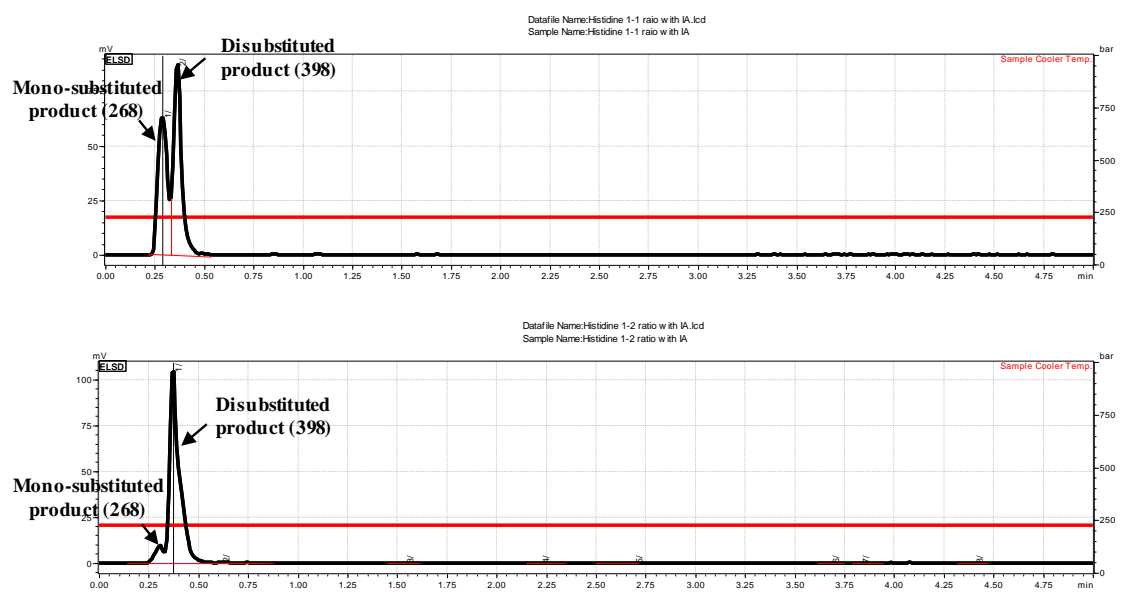

Figure 4.15. LC-trace of crude reaction mixture of the reaction between L-histidine and a double molar ratio of itaconic acid (top) and equimolar ratio of itaconic acid (bottom).

Similarly, the LC chromatogram of the reaction mixture of L-histidine and (a double molar ratio) itaconic acid, highlights the ability of the imidazole moiety to participate in the aza-Michael addition reaction (Figure 4.15). The presence of both the $\boldsymbol{L}$-his-PD product and its disubstituted alternative which did undergo an additional aza-Michael addition $(\mathrm{m} / \mathrm{z}=268$ and $398 \mathrm{~g} / \mathrm{mol}$, respectively), is in line with the findings from NMR analysis (Figure 4.14). Further cyclization of the disubstituted product depicted in Figure 4.14 was not plausible, as this would result in the formation of a quaternary amine. 
In general, very similar results are obtained for the other reactions although some additional side reactions are observed, depending on the used amino acid. For example, we observe dimerization occurring parallel to the aza-Michael addition for almost all amino acids (scheme 4.3, bottom reaction). Furthermore, the formation of intermediate $\mathrm{B}$ and the ensuing imide side-products are detected from LC-MS analysis for most amino acids, though these are not observed after purification. In the case of $L$-DOPA, we observe thermal degradation (oxidized form) during synthesis (resulting in a strong discoloration), effectively generating a diverse mixture of side products as indicated by LC-MS analysis (Appendix C, Figure C7). Although ${ }^{1} \mathrm{H}-$ NMR analysis indicates that the desired $L$-DOPA-PD is present in the reaction mixture, we were unable to isolate it from the reaction mixture and therefore excluded it from further study. In the case of both $L$-lysine and $L$-histidine we observe that the reactions conducted with double molar ratio of itaconic acid (to enforce a double azaMichael addition reaction with the goal to develop tri- or multifunctional carboxylic acids, of which the data is provided in Table 4.2) resulted in di-substituted products (as major component) along with mono substituted product(as minor component). Even though the reaction proceeds well for both monomers, purification remained challenging, likely resulting from the fact that a mixture of both mono and disubstituted products was obtained. Accordingly, these products were obtained in low isolated yields. Nevertheless, the obtained monomer mixtures proved useful for polymerization of thermosets, as will be discussed briefly in the next section. Despite limitations by thermal stability, amino-acid oligomerization, and amide/imide formation, these findings clearly demonstrate that the aza-Michael cascade reaction can be conducted on naturally occurring amino acids to yield the desired multifunctional pyrrolidone monomers after purification.

It is worthwhile to note that significantly higher yields are obtained for reactions conducted on amino acids without a stereocenter. For example, the reaction products $\boldsymbol{\gamma}$-aba-PD (using $\gamma$-aminobutyric acid as reactant) and 11-ada-PD (using 11-aminoundecanoic acid as reactant) are isolated in $98 \%$ and $93 \%$ yield, respectively. In contrast, the products bearing a stereocenter in the amino acid, i.e. $\boldsymbol{L}$ ph-ala-PD and $\boldsymbol{L}$-tyr-PD are obtained in yields between 50 and $70 \%$. Ignoring the presence of side- and intermediate products, we consider it likely that the presence of two stereo centers in these reaction products (one in the amino acid spacer and one in the pyrrolidone ring) frustrates the crystallization during purification and/or promotes dissolution during trituration. Despite the progress of the reaction, purification is the limiting step in this protocol. 


\subsubsection{Thermal ring opening addition polymerization with bis(2- oxazolines).}

In order to polymerize the synthesized pyrrolidone based monomers with bis(2-oxazolines) they should exhibit a sufficiently high thermal stability and low volatility to be subjected to the thermal curing temperature of $180{ }^{\circ} \mathrm{C}$. To evaluate which monomers were suitable for polymerization, thermogravimetric analysis was performed (Table 4.2). Indeed, most monomers, except for $\boldsymbol{L}$-lys-PD and $\boldsymbol{L}$-his-PD proved thermally stable, identified by the temperature where $10 \mathrm{wt} \%$ mass loss occurred ( $T_{90}$, Table 4.2, Appendix C, Figure C10) well above $200{ }^{\circ} \mathrm{C}$. Both $\boldsymbol{L}$-lysPD and $\boldsymbol{L}$-his-PD degraded rapidly when heating beyond $150{ }^{\circ} \mathrm{C}$, effectively placing limits on the polymerization temperature. To evaluate the polymerizability of the developed monomers, a series of (co-)polymers (P1-P15, Table 4.3) has been synthesized in bulk; the desired dicarboxylic acids were mixed with 1,3-bis(4,5dihydrooxazol-2-yl)benzene (IAox) in an equimolar ratio, ground and heated for 30 minutes at the desired reaction temperature. The resulting molecular weights and the glass transition temperature $\left(T_{\mathrm{g}}\right)$ of the ensuing amorphous polymers are listed in table 4.3. Note, as expected, both polymers containing $L$-lys-PD (P14) and $L$-his-PD (P15) cross-linked rapidly during polymerization resulting from the presence of the multifunctional carboxylic acids as side products and could not be subjected to GPC analysis due to their insoluble nature.

In general, we observe that the polymerization proceeds for all other dicarboxylic acids to yield polymers with a molecular weight around $10 \mathrm{~kg} / \mathrm{mol}$ or higher within half an hour of reaction, according to HFIP-GPC analysis. Note, the dispersity of all polymers is relatively broad, which is common for these types of polymers as sometimes low molecular weight components can remain present (cyclics and oligomers). Though such low molecular weight species can be removed by dissolution and precipitation of the polymer, this would require excessive solvent usage and would result in material losses. Instead, we analyzed and used the materials as obtained directly after thermal polymerization in bulk. As reported in previous work $^{28}$, an increase in reaction time generally results in a further increase in molecular weight, however, not by chain-extension via reaction between 2-oxazoline and carboxylic acid moieties ( $k_{1}$, scheme 4.2$)$, but mainly by branching resulting from the reaction between 2-oxazolins and the generated amide bonds $\left(k_{3}\right.$, scheme 4.2$)$.

Furthermore, in the scenario when using $\boldsymbol{L}$-tyr-PD, one can foresee that prolonged polymerization times can facilitate a second side-reaction as the thermal ring opening addition reaction between the aromatic alcohol of the $\boldsymbol{L}$-tyr-PD and the 2-oxazoline moiety yields ether-amide branches ( $k_{2}$, scheme 4.2). Accordingly, to minimize branching and the depletion of the aromatic alcohol groups of $\boldsymbol{L}$-tyr-PD 
during polymerization, we have limited the polymerization time 30 minutes in this study. However, if one aims to develop high molecular weight polymers or thermosets using this type of chemistry, one can consider the use of an excess of bis(2-oxazolines) combined with prolonged reaction times.

Table 4.3. Overview of the reaction temperature, molecules weight, distribution, and glass transition temperature of the polymers developed in this study.

\begin{tabular}{|c|c|c|c|c|c|}
\hline Name & Dicarboxylic acid & $\begin{array}{c}\text { Reaction } \\
\text { temperature } \\
\left({ }^{\circ} \mathbf{C}\right)\end{array}$ & $\begin{array}{c}M_{\mathrm{w}} \\
(\mathrm{kg} / \mathrm{mol})\end{array}$ & Đ (-) & $\begin{array}{c}T_{\mathrm{g}} \\
\left({ }^{\circ} \mathrm{C}\right)\end{array}$ \\
\hline P1 & Sebacic acid & 180 & 38.0 & 8.0 & 37 \\
\hline $\mathbf{P 2}$ & $\begin{array}{c}\text { 75\% Sebacic acid / 25\% } \\
\boldsymbol{L} \text {-ala-PD }\end{array}$ & 180 & 52.3 & 12.1 & 48 \\
\hline $\mathbf{P 3}$ & $L$-ala-PD & 180 & 14.8 & 7.7 & 91 \\
\hline $\mathbf{P 4}$ & $\beta$-ala-PD & 180 & 24.3 & 5.4 & 66 \\
\hline P5 & $\gamma$-aba-PD & 180 & 86.7 & 13.3 & 77 \\
\hline P6 & 11-und-PD & 180 & 12.4 & 7.8 & 42 \\
\hline P7 & ph-ala-PD & 180 & 43.4 & 6.2 & 92 \\
\hline $\mathbf{P 8}$ & $\begin{array}{c}75 \% \text { L-ph-ala-PD / } 25 \% \\
\text { L-tyr-PD }\end{array}$ & 180 & 38.2 & 7.2 & 104 \\
\hline P9 & $\begin{array}{c}50 \% \text { L-ph-ala-PD / 50\% } \\
\text { L-tyr-PD }\end{array}$ & 180 & 19.9 & 9.2 & 110 \\
\hline P10 & $\begin{array}{c}25 \% \text { L-ph-ala-PD } / 75 \% \\
\text { L-tyr-PD }\end{array}$ & 180 & 9.3 & 9.0 & 111 \\
\hline P11 & $L$-tyr-PD & 180 & 7.3 & 8.8 & 107 \\
\hline P12 & $L$-ph-ala-PD & $180^{\mathrm{B}}$ & 20.0 & 5.6 & 84 \\
\hline $\mathbf{P 1 3}$ & $\begin{array}{c}\text { 75\% L-ph-ala-PD / } 25 \% \\
\text { L-tyr-PD }\end{array}$ & $180^{\mathrm{B}}$ & 20.2 & 8.2 & 109 \\
\hline P14 & $L$-lys-PD & 130 & $\overline{\mathrm{A}}$ & $\mathrm{A}$ & 53 \\
\hline P15 & $L$-his-PD & 130 & $\mathrm{~A}$ & $\bar{A}$ & 111 \\
\hline
\end{tabular}

${ }^{\mathrm{A}}$ Cross-linked ${ }^{\mathrm{B}}$ Polymerization conducted for 10 minutes. 
With respect to the thermal behavior, as expected, the glass transition temperature $\left(T_{\mathrm{g}}\right)$ of the polymers increases with the introduction of the pyrrolidone ring, as is observed when comparing the $T_{g}$ of polymer P1 and P6 (while ignoring effects of the molecular weight difference). Retrospectively, decreasing the number of methylene groups in the used dicarboxylic acid increases the $T_{\mathrm{g}}$ further to $77^{\circ} \mathrm{C}$ (P5), and $91{ }^{\circ} \mathrm{C}(\mathbf{P 3})$. Note, though we would expect $\mathbf{P 4}$ to have a higher $T_{\mathrm{g}}$ than $\mathbf{P 5}$ resulting from the decreased number of methylene spacers in the dicarboxylic acid, we expect that the $T_{\mathrm{g}}$ difference originates from the drastic difference in $M_{\mathrm{w}}$ between the two polymers. The introduction of pendant phenyl or phenol groups in addition to the pyrrolidone moiety in the polymer backbone pushes the $T_{\mathrm{g}}$ beyond $90{ }^{\circ} \mathrm{C}$, as is clearly observed for the $T_{\mathrm{g}}$ of polymers P7 to P11. Similarly, the $T_{\mathrm{g}}$ of the developed polymer increases dramatically from $53{ }^{\circ} \mathrm{C}$ to $111{ }^{\circ} \mathrm{C}$ when exchanging the pendant aliphatic amine functionality (using $\boldsymbol{L}$-lys-PD, P14) with an aromatic imidazole functionality (using $L$-his-PD, P15), respectively.

Interestingly, only polymer $\mathbf{P 8}$ and $\mathbf{P 9}$, which contain large amounts of $\boldsymbol{L}$ tyr-PD as dicarboxylic acid show a molecular weight $\left(M_{\mathrm{w}}\right)$ below $10 \mathrm{~kg} / \mathrm{mol}$. The origin of this behavior is found in the slow thermal decarboxylation of this monomer over time (Figure 4.17), effectively generating mono-functional carboxylic acids, which function as chain stoppers and thereby hamper the molecular weight buildup (Figure 4.16). Please note that $\boldsymbol{L}$-ph-ala-PD also undergoes decarboxylation at higher temperature, although the rate of decarboxylation is not significant at the employed temperature. The effect of this side-reaction becomes evident when monitoring the ratio of ring-opened 2-oxazoline (conversion determined using ${ }^{1} \mathrm{H}$ NMR is shown in Appendix C, Figure C9) over time during polymerization via ${ }^{1} \mathrm{H}-\mathrm{NMR}$ analysis in the presence of increasing amount of $\boldsymbol{L}$-tyr-PD and $\boldsymbol{L}$-ph-ala-PD (figure 4.16, left). As reported in literature, we observe that the reaction proceeds rather quickly at $180{ }^{\circ} \mathrm{C}$ and achieves $>90 \%$ conversion within 10 minutes of reaction time ${ }^{28,32}$. However, depletion of 2-oxazoline rings proceeds significantly slower with increasing amount of $\boldsymbol{L}$-tyr-PD present in the system, suggesting that $\boldsymbol{L}$-tyr-PD has a lowered reactivity than the $\boldsymbol{L}$-ph-ala-PD. For example, when comparing P7 (100\% $\boldsymbol{L}$-ph-ala-PD) with P11 (100\% L-tyr-PD) we observe a 2-oxazoline conversion of $85 \%$ and $46 \%$, respectively after one minute of reaction time. Furthermore, after 30 minutes of reaction, we observe that an increasing concentration of unreacted 2-oxazoline moieties remain present in systems with increasing amount of $\boldsymbol{L}$-tyr-PD. To illustrate, the remaining percentage of 2-oxazoline moieties present increases from $1 \%$ for $\mathbf{P 7}$ to $2 \%$ for P8 and P9, and 5\% for P10 and P11, suggesting that an increasing amount of $\boldsymbol{L}$-tyr-PD has degraded during the reaction. The normalized GPC traces of the polymers P7-P11 obtained after 30 minutes of polymerization are displayed in Figure 4.16 , right. As the degradation of $\boldsymbol{L}$-tyr-PD becomes more dominant, the presence of low molecular weight species increases and the overall molecular weight decreases. 
Indeed, these findings suggest the presence of an increased number of chains that are unable to participate in the polymerization process as the terminal $\boldsymbol{L}$-tyr-PD has degraded. Although the thermal lability of $\boldsymbol{L}$-tyr-PD makes its direct use in polymerization challenging, it appears to be successfully incorporated in the polymeric backbone when used in lowered concentration and in combination with $\boldsymbol{L}$ ph-ala-PD (P10, 25\% L-tyr-PD). Although degradation of the $\boldsymbol{L}$-tyr-PD is still expected to proceed under these conditions, it does not appear to significantly limit the molecular weight build-up as the resulting polymer has a molecular weight close to $40 \mathrm{~kg} / \mathrm{mol}$. Overall, these findings confirm that the synthesis of polymers bearing functional side groups (phenol groups in this case) is possible in one reaction step in bulk using 2-oxazoline chemistry.
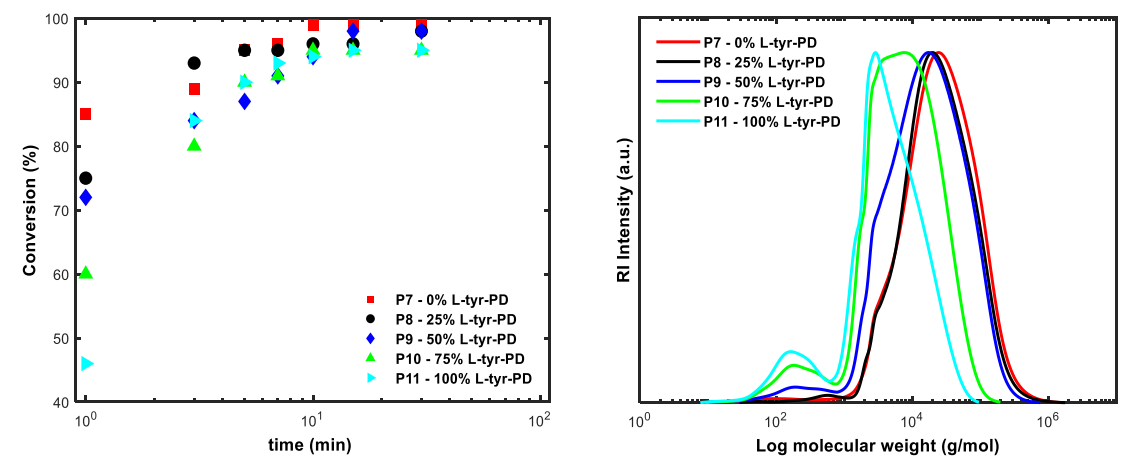

Figure 4.16. Left, 2-oxazoline conversion, obtained from ${ }^{1} \mathrm{H}$-NMR analysis, during polymerization at $180^{\circ} \mathrm{C}$ in polymers containing varying amount of $\boldsymbol{L}$-tyr-PD and $\boldsymbol{L}$ ph-ala-PD (P7-P11). Right, GPC traces of the ensuing polymers obtained after 30 minutes of reaction time. It is visible how with increase L-tyr content the molecular weight built-up is hampered.

\subsubsection{Evidence for thermal decarboxylation of $L$-tyr-PD}

$100 \mathrm{mg}$ of the synthesized L-tyr-PD monomer was loaded in glass vial and heated under nitrogen atmosphere at $180{ }^{\circ} \mathrm{C}$ for 30 minutes in an oil bath. Both the virgin and heated $\boldsymbol{L}$-tyr-PD are analyzed using LC-MS. Figure 4.17 shows the corresponding LC elugram with the masses of the peaks provided. 


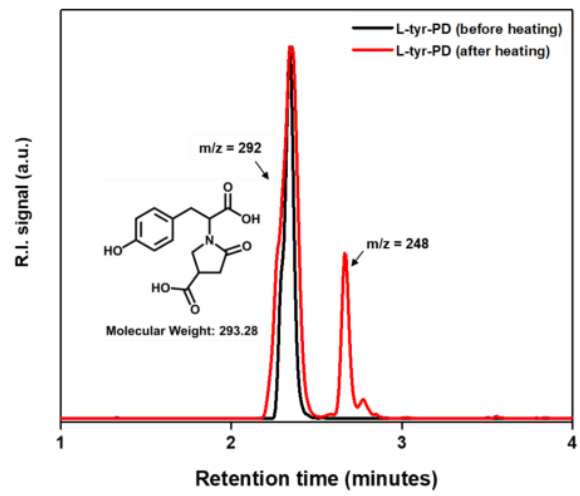

Figure 4.17. LC elugram depicting the rise of a compound having a $\mathrm{m} / \mathrm{z}$ of $249 \mathrm{~g} / \mathrm{mol}$ (corresponding to the loss of $\mathrm{CO}_{2}$ having a weight of $44 \mathrm{~g} / \mathrm{mol}$ ) upon prolonged heating of $\mathbf{L}$-tyr-PD.

The figure 4.17 shown above, depicts that indeed decarboxylation happens when $100 \% \boldsymbol{L}$-tyr-PD is used, whereas similar tests are performed on $\boldsymbol{L}$-ph-ala-PD and no significant changes are observed.

\subsubsection{Rheology and stress relaxation}

From the above section it is clear that pyrrolidone based dicarboxylic acids obtained from naturally occurring amino acids can readily be developed and polymerized with bis(2-oxazolines). Though the thermal transitions can be controlled by careful selection of the dicarboxylic acid, the influence of the spacer length and functionality of the pendant group on the rheological behavior of the ensuing polymers is unknown. Therefore, to identify the effect of 1) the pyrrolidone ring, 2) pendant phenyl moiety, and 3) pendant phenol moiety on the viscoelastic behavior of the polymers, the temperature dependency and stress relaxation behavior of polymers P1, P2, P12 and P13 have been evaluated. To identify the effect of the pyrrolidone moiety in the polymer backbone, both P1, based on sebacic acid, and P2, containing $25 \%$ L-ala-PD and $75 \%$ sebacic acid have been selected as model polymers. As can be deduced from Figure 4.18A and Table 4.3, P2 has a molecular weight roughly $37 \%$ higher than $\mathbf{P 1}$ and exhibits a $T_{\mathrm{g}} 10^{\circ} \mathrm{C}$ higher than that of P1. Both parameters are known to affect the rheological behavior of polymer melts; the viscosity of polymers melts generally increases during cooling displaying an exponential increase, until the glass transition temperature is reached ${ }^{41}$. Therefore, when cooling polymers from $200{ }^{\circ} \mathrm{C}$, the viscosity of polymers with a higher $T_{\mathrm{g}}$ increases more rapidly. Secondly, the relaxation of polymer chains increase with increasing molecular weight, thereby affecting the viscosity of the system following the relation 
$\eta_{0} \sim M_{\mathrm{w}}$ for non-entangled polymers (likely the case for polymers evaluated in this study given their low molecular weight and the relatively rigid backbone structures, as shown in the (Appendix C, Figure C10). Accordingly, an increase in viscosity and relaxation times may be expected when evaluating polymers with increasing molecular weights. Indeed, as is observed from Figure 4.18B, when comparing the temperature dependency of the elastic modulus of $\mathbf{P 1}$ and P2, we observe comparable behavior with roughly a $15{ }^{\circ} \mathrm{C}$ temperature difference, possibly originating from the difference in the $T_{\mathrm{g}}$ between the two polymers. However, while the elastic modulus of $\mathbf{P 1}$ displays the expected exponential type of behavior during cooling, polymer $\mathbf{P 2}$ displays deviation from such behavior between the temperatures of 120 and $170{ }^{\circ} \mathrm{C}$ (also present in the loss modulus, figure 4.20 and is clearly observed after performing a $T_{\mathrm{g}}$ correction for the polymers (Figure 4.19 left). Such a deviation from exponential type of behavior can correspond to the presence of the onset of an (entanglement) network caused by either high molecular weight polymer chains, or additional interactions existing in the polymer melt (i.e. hydrogen bonding of amide bonds with the pyrrolidone carbonyls), both resulting in an enhancement of relaxation times.

A)
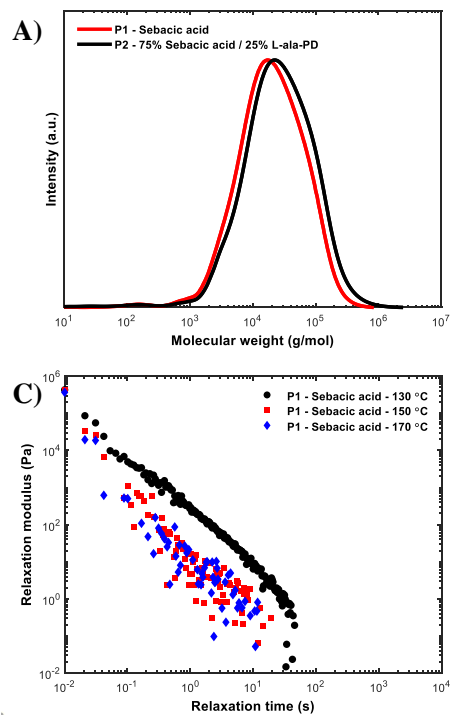
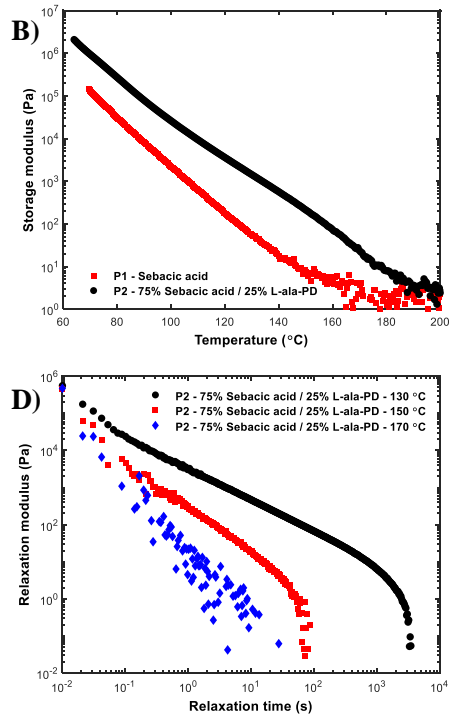

Figure 4.18. A) GPC traces of polymer P1 and P2. B) Temperature dependency of the elastic modulus of polymer $\boldsymbol{P 1}$ and $\mathbf{P 2}$, determined at a strain of $0.5 \%$ and $a$ frequency of $1 \mathrm{rad} \mathrm{s}^{-1}$ during cooling at a rate of $5^{\circ} \mathrm{C} / \mathrm{min}$. C) and D) Relaxation spectra taken at $130{ }^{\circ} \mathrm{C}, 150^{\circ} \mathrm{C}$, and $170{ }^{\circ} \mathrm{C}$ for polymers $\boldsymbol{P 1}$ and $\boldsymbol{P} 2$ respectively. Note, settings used for acquisition of the relaxation spectra are provided in the experimental section. 
Indeed, when comparing the relaxation moduli of $\mathbf{P 1}$ and $\mathbf{P 2}$ determined at 130, 150, and $170{ }^{\circ} \mathrm{C}$ we observe that $\mathbf{P 2}$ exhibits a delayed relaxation behavior at the respective temperatures, corresponding to the enhanced elastic modulus over the whole temperature range. However, when correcting for the difference in $T_{\mathrm{g}}$ of the materials, one can observe that the relaxation become rather similar with the exception that $\mathbf{P 2}$ takes longer to relax (Figure 4.19 right).
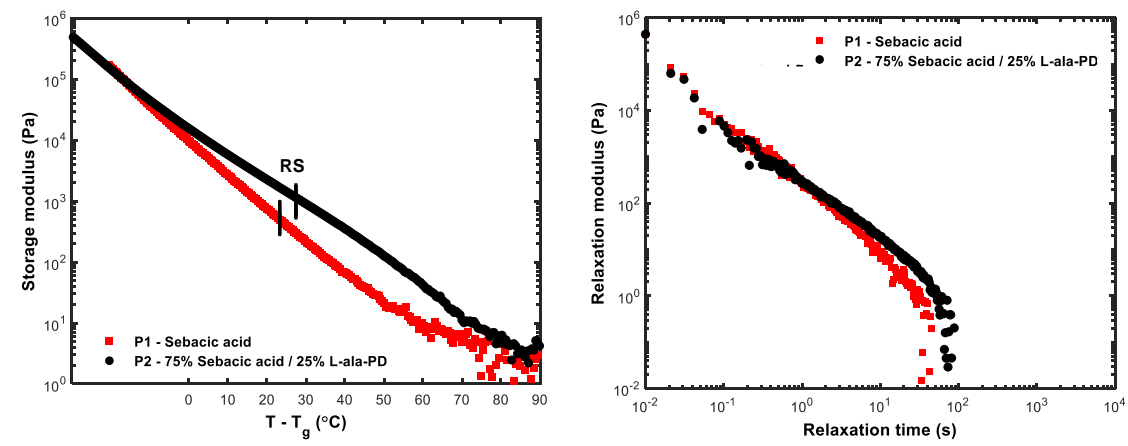

Figure 4.19. Temperature dependency of the storage modulus of $\boldsymbol{P} 1$ and $\mathbf{P 2}$, after performing a $T_{g}$ correction. Right, comparison of the relaxation spectra of $\boldsymbol{P} \mathbf{1}$ and $\mathbf{P} \mathbf{2}$ taken in the same $T$ - $T_{g}$ temperature range denoted as $R S$ in the left image (and taken at actual temperature of $130{ }^{\circ} \mathrm{C}$ for $\boldsymbol{P 1}$ and $150{ }^{\circ} \mathrm{C}$ for $\boldsymbol{P 2}$ ).

This suggests that the difference in elastic modulus between the P1 and P2 are likely the result from the difference in molecular weight and $T_{\mathrm{g}}$ and not from the presence of the pyrrolidone moiety. In other words, even though the pyrrolidone moiety is a hydrogen bonding acceptor, it does not seem to significantly influence the viscoelastic properties of the developed polymers. Note, an overview of the loss moduli of $\mathbf{P 1}$ and $\mathbf{P 2}$ are shown in figure 4.20.

In order to identify the effect of $\boldsymbol{L}$-ph-ala-PD and $\boldsymbol{L}$-tyr-PD in the polymer backbone, two new polymers (P12 and P13) are synthesized with a comparable $M_{\mathrm{w}}$. Given that the molecular weight and $T_{\mathrm{g}}$ strongly affect the viscoelastic behavior, as is observed for $\mathbf{P 1}$ and $\mathbf{P 2}$, the reaction time has been set to 10 minutes yielding polymers both having a $M_{\mathrm{w}}$ of $20 \mathrm{~kg} / \mathrm{mol}$. However, as can be deduced from Figure 4.22A, polymer P13 contains a higher fraction of low molecular weight species, resulting in a broader dispersity. Note that the $T_{\mathrm{g}}$ of $\mathbf{P 1 2}\left(86^{\circ} \mathrm{C}\right)$ is lower than the $T_{\mathrm{g}}$ of $\mathbf{P 7}\left(92^{\circ} \mathrm{C}\right)$, likely resulting from the molecular weight difference between these polymers. 

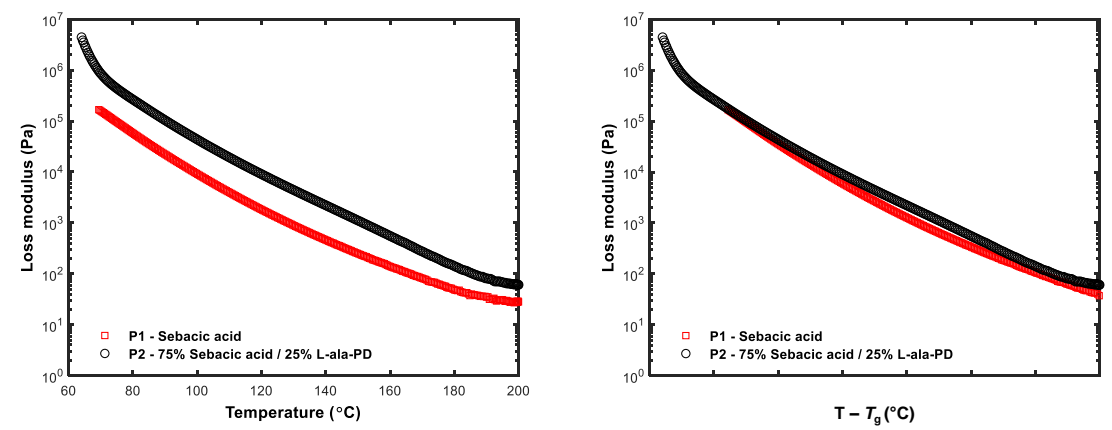

Figure 4.20. Temperature dependency of the elastic modulus of polymer $\boldsymbol{P 1}$ and $\boldsymbol{P 2}$ determined at a strain of $0.5 \%$ and a frequency of 1 rad $\mathrm{s}^{-1}$ during cooling at a rate of $5{ }^{\circ} \mathrm{C} / \mathrm{min}$, determined before (left) and after (right) $T_{g}$ correction.

In addition, low molecular weight oligomers act as a plasticizer in pyrrolidone based polymers, potentially contributing to the $T_{\mathrm{g}}$ suppression as is observed in earlier work ${ }^{42}$. Based on DSC analysis, polymers P12 and P13 exhibit a $T_{\mathrm{g}}$ difference of roughly $20{ }^{\circ} \mathrm{C}$, corresponding well with the $T_{\mathrm{g}}$ difference observed in rheology (peak in loss modulus, figure 4.21).
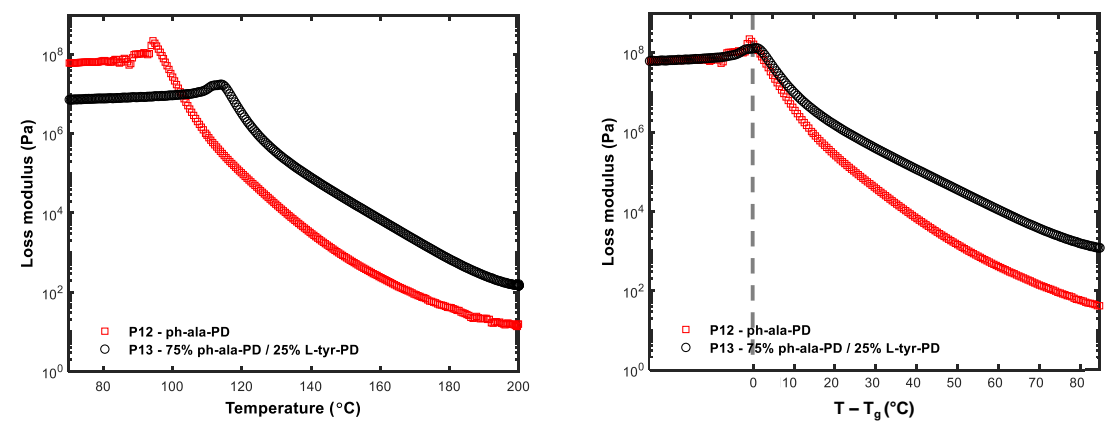

Figure 4.21. Temperature dependency of the elastic modulus of polymer P12 and $P 13$ determined at a strain of $0.5 \%$ and a frequency of $1 \mathrm{rad} \mathrm{s}^{-1}$ during cooling at a rate of $5{ }^{\circ} \mathrm{C} / \mathrm{min}$, determined before (left) and after (right) $T_{g}$ correction.

When comparing the temperature dependency of the elastic modulus of the polymers upon cooling (Figure 4.22B), we observe that both materials deviate from exponential type of behavior, whereas P13 shows this behavior over a broader temperature range between $130-180{ }^{\circ} \mathrm{C}$ compared to $100-130{ }^{\circ} \mathrm{C}$ for $\mathbf{P 1 2}$. In contrast to earlier observations for $\mathbf{P 1}$ and $\mathbf{P 2}$, the relaxation spectra of $\mathbf{P 1 2}$ are significantly different in this temperature region. Though the relaxation spectrum of 
P12 taken at $110{ }^{\circ} \mathrm{C}$ displays very slow relaxation of the sample, we consider this effect the result from the sample being too close to the glass transition temperature and hence having long relaxation times. Instead, the relaxation spectrum taken at 130 ${ }^{\circ} \mathrm{C}$ clearly shows the presence of two separate relaxation processes, one which takes place within the first 10 seconds whereas the second process requires roughly 1000 seconds to relax. Such behavior suggests the presence of an additional process occurring in the melt with a relatively fast relaxation time. Given the difference in chemical structure between P1, P2 and P12, we do not consider hydrogen bonding, nor the presence of pyrrolidone ring the cause of this behavior. Instead, we consider it likely that either this enhancement in stress relaxation originates from the differences in chain rigidity or potential pi-pi stacking between the pendant phenyl rings of the $\boldsymbol{L}$-ph-ala-PD moieties. This behavior is lost when maintaining P12 at temperature of $150{ }^{\circ} \mathrm{C}$ and higher; the corresponding stress relaxation spectra show that this process relaxes within 1 second, which corresponds to the frequency of the temperature ramp used to generate the data displayed in Figure 4.22B. In other words, though the process still takes place, it readily relaxes during the experiment and hence is not picked up.

A)
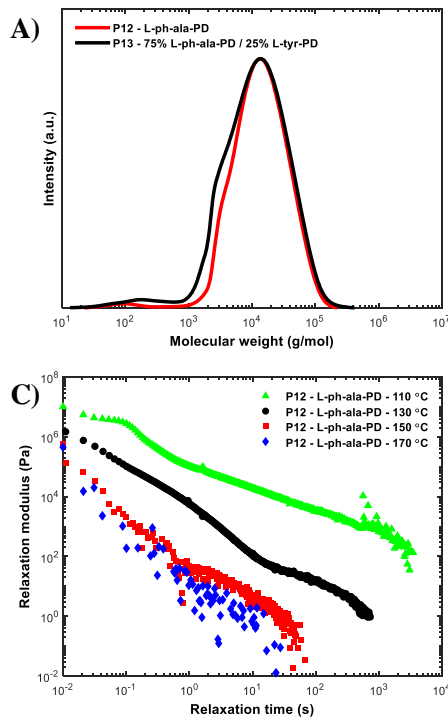

B)
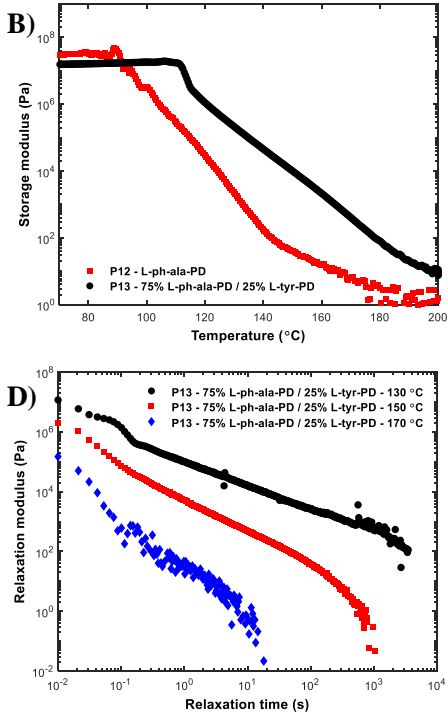

Figure 4.22. A) GPC traces of polymer P12 and P13. B) Temperature dependency of the elastic modulus of polymer P12 and P13, determined at a strain of $0.5 \%$ and $a$ frequency of $1 \mathrm{rad} \mathrm{s}^{-1}$ during cooling at a rate of $5^{\circ} \mathrm{C} / \mathrm{min}$. C) and D) Relaxation spectra taken at $110^{\circ} \mathrm{C}(\boldsymbol{P 1 2})$ and at $130{ }^{\circ} \mathrm{C}, 150{ }^{\circ} \mathrm{C}$, and $170{ }^{\circ} \mathrm{C}$ for polymers $\mathrm{P12}$ and $\mathbf{P 1 3}$ respectively. Note, the same experimental protocols were followed as used in Figure 4.19, which are provided in the experimental section. 
As mentioned earlier, the introduction of $\boldsymbol{L}$-tyr-PD in the polymer backbone broadens the temperature range where the elastic and loss modulus (figure 4.22 and 4.21) deviate from exponential behavior. We expect that this originates from the presence of the pendant phenol groups of $\boldsymbol{L}$-tyr-PD, which provide additional hydrogen donors for hydrogen bonding to the polymer. Therefore, polymer P13 is expected to have a significantly higher hydrogen bonding density than the previously explored polymers, which is expected to remain present at higher temperatures/longer timescales. The stress relaxation spectra of P13 are different from those of P12 and do not show a two-stage relaxation process (Figure 4.22D). This is likely the result of the combined relaxation mechanisms including pi-pi interactions and the increased hydrogen bonding density in the system. This behavior becomes more apparent when performing a $T_{\mathrm{g}}$ correction for both polymers, which highlights the higher elastic modulus of P13 above $T_{\mathrm{g}}$ (Figure 4.23, left). Furthermore, the overall relaxation time does not seem to be influenced strongly by the presence of $\boldsymbol{L}$-tyr-PD, the difference in relaxation behavior becomes apparent when comparing $T_{\mathrm{g}}$ corrected relaxation spectra (Figure 4.23, right). Overall, these preliminary findings clearly demonstrate the potential of the bis(2-oxazolines) curing chemistry as it allows for the bulk synthesis of thermoplastic polymers with controllable relaxation behavior via tailoring of the hydrogen bonding density (dynamic network behavior).
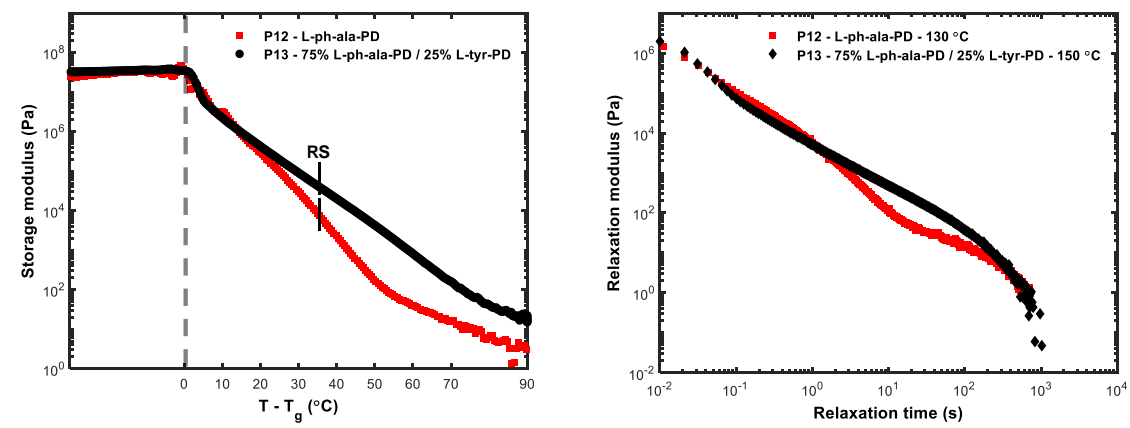

Figure 4.23. Temperature dependency of the storage modulus of $\boldsymbol{P 1 2}$ and P13, after $T_{g}$ correction. Right, comparison of the relaxation spectra of $\boldsymbol{P} 12$ and $\boldsymbol{P} 13$ taken $T$ $T_{g}+35^{\circ} \mathrm{C}$ (denoted as $R S$ in left image, taken at actual temperatures of $130^{\circ} \mathrm{C}$ for $\boldsymbol{P 1 2}$ and $150{ }^{\circ} \mathrm{C}$ for $\left.\boldsymbol{P 1 3}\right)$.

\subsection{Conclusions}

The successful synthesis of renewable pyrrolidone based dicarboxylic acids using naturally occurring $L$-amino acids and itaconic acids is reported in this work. The monomers are synthesized via the cascade aza-Michael addition/cyclization reaction and are isolated after crystallization or trituration with isolated yields 
between 50 and $70 \%$. Though synthesis of pyrrolidone functionalized monomers bearing pendant phenol groups was feasible, the purification of monomers bearing pendant amine or imidazole groups proved challenging, as these functional groups are also susceptible towards aza-Michael addition reactions. Overall, the synthesized monomers with side groups proves excellent candidates for thermal curing in 2oxazoline based resins in bulk at temperatures of 130 or $180^{\circ} \mathrm{C}$ to yield thermoplastic polymer or thermosets. GPC analysis on the non-crosslinked materials confirms that all polymers are obtained in molecular weights above $10 \mathrm{~kg} / \mathrm{mol}$, except for polymers containing large amounts of $\boldsymbol{L}$-tyr-PD, which undergoes thermal degradation during thermal curing. Nevertheless, copolymerization of $\boldsymbol{L}$-tyr-PD (25\% of dicarboxylic acid component) with $\boldsymbol{L}$-ph-ala-PD (75\%) proved beneficial in developing a polymer with an $M_{\mathrm{w}}$ of $40 \mathrm{~kg} / \mathrm{mol}$ while bearing one pendant phenol group for every four dicarboxylic acid residues. Evaluation of the relaxation behavior and temperature dependency of the viscoelastic behavior suggests an increase in viscosity and change in relaxation behavior when introducing $\boldsymbol{L}$-tyr-PD in the polymer backbone. This behavior confirms the presence of the functional (phenolic-OH) group of $\boldsymbol{L}$-tyr-PD as free (pendant) group, since its presence effectively increases the overall hydrogen bonding density of the polymer melt. Furthermore, it is expected that the developed monomers and polymers can be subjected to post-functionalization, providing additional control over the thermo-mechanical performance and viscoelastic behavior.

For further information, please refer to Appendix C. 


\subsection{References}

(1) Yao, K.; Tang, C. Controlled Polymerization of Next-Generation Renewable Monomers and Beyond. Macromolecules 2013, 46 (5), 1689-1712. https://doi.org/10.1021/ma3019574.

(2) Miller, S. A. Sustainable Polymers: Opportunities for the next Decade. ACS Macro Lett. 2013, 2 (6), 550-554. https://doi.org/10.1021/mz400207g.

(3) Lebarbé, T.; Maisonneuve, L.; Nga Nguyen, T. H.; Gadenne, B.; Alfos, C.; Cramail, H. Methyl 10-Undecenoate as a Raw Material for the Synthesis of Renewable Semi-Crystalline Polyesters and Poly(Ester-Amide)S. Polym. Chem. 2012, 3 (10), 2842-2851.

https://doi.org/10.1039/c2py20394d.

(4) Meier, M. A. R.; Metzger, J. O.; Schubert, U. S. Plant Oil Renewable Resources as Green Alternatives in Polymer Science. Chem. Soc. Rev. 2007, 36 (11), 1788-1802. https://doi.org/10.1039/b703294c.

(5) Ma, S.; Li, T.; Liu, X.; Zhu, J. Research Progress on Bio-Based Thermosetting Resins. Polym. Int. 2016, 65 (2), 164-173. https://doi.org/10.1002/pi.5027.

(6) Maiti, S.; Ray, S. S.; Kundu, A. K. Rosin: A Renewable Resource for Polymers and Polymer Chemicals. Prog. Polym. Sci. 1989, 14 (3), 297-338. https://doi.org/10.1016/00796700(89)90005-1.

(7) A. Gandini. Polymer from Renewable Resources: A Challenge for the Furure of Macromolecular Materials. Macromolecules 2008, 41 (24), 9491-9504.

https://doi.org/10.1021/ma801735u.

(8) Alvès, M. H.; Sfeir, H.; Tranchant, J. F.; Gombart, E.; Sagorin, G.; Caillol, S.; Billon, L.; Save, M. Terpene and Dextran Renewable Resources for the Synthesis of Amphiphilic Biopolymers. Biomacromolecules 2014, 15 (1), 242-251. https://doi.org/10.1021/bm401521f.

(9) Gao, C.; Ma, C.; Xu, P. Biotechnological Routes Based on Lactic Acid Production from Biomass. Biotechnol. Adv. 2011, 29 (6), 930-939. https://doi.org/10.1016/j.biotechadv.2011.07.022.

(10) Okabe, M.; Lies, D.; Kanamasa, S.; Park, E. Y. Biotechnological Production of Itaconic Acid and Its Biosynthesis in Aspergillus Terreus. Appl. Microbiol. Biotechnol. 2009, 84 (4), $597-$ 606. https://doi.org/10.1007/s00253-009-2132-3.

(11) Dai, J.; Ma, S.; Liu, X.; Han, L.; Wu, Y.; Dai, X.; Zhu, J. Synthesis of Bio-Based Unsaturated Polyester Resins and Their Application in Waterborne UV-Curable Coatings. Prog. Org. Coatings 2015, 78, 49-54. https://doi.org/10.1016/j.porgcoat.2014.10.007.

(12) Farmer, T. J.; Castle, R. L.; Clark, J. H.; Macquarrie, D. J. Synthesis of Unsaturated Polyester Resins from Various Bio-Derived Platform Molecules. Int. J. Mol. Sci. 2015. https://doi.org/10.3390/ijms160714912.

(13) Moore, O. B.; Hanson, P.-A.; Comerford, J. W.; Pellis, A.; Farmer, T. J. Improving the PostPolymerization Modification of Bio-Based Itaconate Unsaturated Polyesters: Catalyzing AzaMichael Additions With Reusable Iodine on Acidic Alumina. Front. Chem. 2019, 7 (July), 114. https://doi.org/10.3389/fchem.2019.00501. 
(14) Betancourt, T.; Pardo, J.; Soo, K.; Peppas, N. A. Characterization of PH-Responsive Hydrogels of Poly(Itaconic Acid-g-Ethylene Glycol) Prepared by UV-Initiated Free Radical Polymerization as Biomaterials for Oral Delivery of Bioactive Agents. J. Biomed. Mater. Res. Part A 2010, 93 (1), 175-188. https://doi.org/10.1002/jbm.a.32510.

(15) Bednarz, S.; Półćwiartek, K.; Wityk, J.; Strachota, B.; Kredatusová, J.; Beneš, H.; Wesołowska-Piętak, A.; Kowalski, G. Polymerization-Crosslinking of Renewable Itaconic Acid in Water and in Deep Eutectic Solvents. Eur. Polym. J. 2017, 95 (July), 241-254. https://doi.org/10.1016/j.eurpolymj.2017.08.020.

(16) Bednarz, S.; Wesołowska-Piętak, A.; Konefał, R.; Świergosz, T. Persulfate Initiated FreeRadical Polymerization of Itaconic Acid: Kinetics, End-Groups and Side Products. Eur. Polym. J. 2018, 106 (April), 63-71. https://doi.org/10.1016/j.eurpolymj.2018.07.010.

(17) Winkler, M.; Lacerda, T. M.; Mack, F.; Meier, M. A. R. R. Renewable Polymers from Itaconic Acid by Polycondensation and Ring-Opening-Metathesis Polymerization. Macromolecules 2015, 48 (5), 1398-1403. https://doi.org/10.1021/acs.macromol.5b00052.

(18) Dai, J.; Ma, S.; Wu, Y.; Han, L.; Zhang, L.; Zhu, J.; Liu, X. Polyesters Derived from Itaconic Acid for the Properties and Bio-Based Content Enhancement of Soybean Oil-Based Thermosets. Green Chem. 2015, 17 (4), 2383-2392. https://doi.org/10.1039/c4gc02057j.

(19) Farmer, T. J.; Comerford, J. W.; Pellis, A.; Robert, T. Post-Polymerization Modification of Bio-Based Polymers: Maximizing the High Functionality of Polymers Derived from Biomass. Polym. Int. 2018, 67 (7), 775-789. https://doi.org/10.1002/pi.5573.

(20) Lv, A.; Cui, Y.; Du, F. S.; Li, Z. C. Thermally Degradable Polyesters with Tunable Degradation Temperatures via Postpolymerization Modification and Intramolecular Cyclization. Macromolecules 2016, 49 (22), 8449-8458.

https://doi.org/10.1021/acs.macromol.6b01325.

(21) Chanda, S.; Ramakrishnan, S. Poly(Alkylene Itaconate)s - An Interesting Class of Polyesters with Periodically Located Exo-Chain Double Bonds Susceptible to Michael Addition. Polym. Chem. 2015, 6 (11), 2108-2114. https://doi.org/10.1039/c4py01613k.

(22) Wang, J.; Li, P.; Choy, Y.; Chan, A. S. C.; Yee, F. Advances and Applications in Organocatalytic Asymmetric Aza-Michael Addition. 2012, 917-925. https://doi.org/10.1002/cctc.201200135.

(23) Bosica, G.; Spiteri, J.; Borg, C. No Title. Tetrahedron 2014, 70 (14), 2449-2454. https://doi.org/10.1016/j.tet.2014.02.021.

(24) Bosica, G.; Debono, A. J. Uncatalyzed, Green Aza-Michael Addition of Amines to Dimethyl Maleate. Tetrahedron 2014, 70 (37), 6607-6612. https://doi.org/10.1016/j.tet.2014.06.124.

(25) Qi, P.; Chen, H.-L. L.; Nguyen, H. T. H.; Lin, C.-C. C.; Miller, S. A. Synthesis of Biorenewable and Water-Degradable Polylactam Esters from Itaconic Acid. Green Chem. 2016, 18 (15), 4170-4175. https://doi.org/10.1039/C6GC01081D.

(26) Nsengiyumva, O.; Miller, S. A. Synthesis, Characterization, and Water-Degradation of Biorenewable Polyesters Derived from Natural Camphoric Acid. 2019, 973-978. https://doi.org/10.1039/c8gc03990a.

Noordzij, G. J.; van den Boomen, Y. J. G.; Gilbert, C.; van Elk, D. J. P.; Roy, M.; Wilsens, C. 
H. R. M.; Rastogi, S. The Aza-Michael Reaction: Towards Semi-Crystalline Polymers from Renewable Itaconic Acid and Diamines. Polym. Chem. 2019, 4049-4058.

https://doi.org/10.1039/c9py00463g.

(28) Roy, M.; Noordzij, G. J. G. J.; Van Den Boomen, Y.; Rastogi, S.; Wilsens, C. H. R. M. R. M. C. H. R. M. Renewable (Bis)Pyrrolidone Based Monomers as Components for Thermally Curable and Enzymatically Depolymerizable 2-Oxazoline Thermoset Resins. ACS Sustain. Chem. Eng. 2018, 6 (4), 5053-5066. https://doi.org/10.1021/acssuschemeng.7b04716.

(29) Ayadi, F.; Mamzed, S.; Portella, C.; Dole, P. Synthesis of Bis(Pyrrolidone-4-Carboxylic Acid)Based Polyamides Derived from Renewable Itaconic Acid- Application as a Compatibilizer in Biopolymer Blends. Polym. J. 2013, 45 (10), 766-774. https://doi.org/10.1038/pj.2012.206.

(30) Ali, M. A.; Tateyama, S.; Kaneko, T. Syntheses of Rigid-Rod but Degradable Biopolyamides from Itaconic Acid with Aromatic Diamines. Polym. Degrad. Stab. 2014, 109 (July), 367-372. https://doi.org/10.1016/j.polymdegradstab.2014.05.031.

(31) Ali, M. A.; Tateyama, S.; Oka, Y.; Kaneko, D.; Okajima, M. K.; Kaneko, T. Syntheses of High-Performance Biopolyamides Derived from Itaconic Acid and Their Environmental Corrosion. Macromolecules 2013, 46 (10), 3719-3725. https://doi.org/10.1021/ma400395b.

(32) Roy, M.; Wilsens, C. H. R. M.; Leoné, N.; Rastogi, S. Use of Bis(Pyrrolidone)-Based Dicarboxylic Acids in Poly(Ester-Amide)-Based Thermosets: Synthesis, Characterization, and Potential Route for Their Chemical Recycling. ACS Sustain. Chem. Eng. 2019, 7 (9), 8842 8852. https://doi.org/10.1021/acssuschemeng.9b00850.

(33) Böhme, F.; Clausnitzer, C.; Gruber, F.; Grutke, S.; Huber, T.; Pötschke, P.; Voit, B. Hyperbranched Poly(Ether Amide)s via Nucleophilic Ring Opening Reaction of Oxazolines. High Perform. Polym. 2001, 13 (2). https://doi.org/10.1088/0954-0083/13/2/303.

(34) Huber, T.; Böhme, F.; Komber, H.; Kronek, J.; Luston, J.; Voigt, D.; Voit, B. New Hyperbranched Poly(Ether Amide)s via Nucleophilic Ring Opening of 2-OxazolineContaining Monomers. Macromol. Chem. Phys. 1999, 200 (1), 126-133. https://doi.org/10.1002/(SICI)1521-3935(19990101)200:1<126::AID-MACP126>3.0.CO;2-O.

(35) Lustoň, J.; Kronek, J.; Böhme, F. Synthesis and Polymerization Reactions of Cyclic Imino Ethers. i. Ring-Opening Homopolyaddition of AB-Type Hydroxyphenyl-Substituted 2Oxazolines. J. Polym. Sci. Part A Polym. Chem. 2006, 44 (1), 343-355. https://doi.org/10.1002/pola.21159.

(36) Wilsens, C. H. R. M.; Wullems, N. J. M.; Gubbels, E.; Yao, Y.; Rastogi, S.; Noordover, B. A. J. Synthesis, Kinetics, and Characterization of Bio-Based Thermosets Obtained through Polymerization of a 2,5-Furandicarboxylic Acid-Based Bis(2-Oxazoline) with Sebacic Acid. Polym. Chem. 2015, 6 (14), 2707-2716. https://doi.org/10.1039/c4py01609b.

(37) Frank, B.; Clausnitzer, C.; Gruber, F.; Grutke, S.; Huber, T.; Petra, P.; Voit, B. Hyperbranched Poly ( Ether Amide ) s via Nucleophilic Ring Opening Reaction of Oxazolines. 2001, 13.

(39) Fazio, M. J. Nucleophilic Ring Opening of 2-Oxazolines with Amines: A Convenient Synthesis for Unsymmetrically Substituted Ethylenediamines. J. Org. Chem. 1984, 49 (25), 4889-4893. https://doi.org/10.1021/jo00199a029. 
(40) Clausnitzer, C.; Voit, B.; Komber, H.; Voigt, D. Poly(Ether Amide) Dendrimers via Nucleophilic Ring-Opening Addition Reactions of Phenol Groups toward Oxazolines: Synthesis and Characterization. Macromolecules 2003, 36 (19), 7065-7074. https://doi.org/10.1021/ma034318y.

(41) Liu, C. Y.; He, J.; Keunings, R.; Bailly, C. New Linearized Relation for the Universal Viscosity - Temperature Behavior of Polymer Melts. Macromolecules 2006, 39 (25), 88678869. https://doi.org/10.1021/ma061969w.

(42) Noordzij, G. J. G. J.; Roy, M.; Bos, N.; Reinartz, V.; Wilsens, C. H. R. M. C. H. R. M. Improving the Hydrolysis Rate of the Renewable Poly(Hexamethylene Sebacate) Through Copolymerization of a Bis(Pyrrolidone)-Based Dicarboxylic Acid. Polymers (Basel). 2019, 11 (10), 1654. https://doi.org/10.3390/polym11101654. 



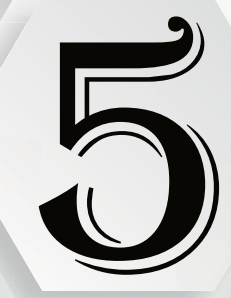

\section{EVALUATING THE PERFORMANCE OF AMIDE BASED ORGANIC COMPOUNDS AS PLASTICIZER AND NUCLEATING AGENT IN POLYLACTIDE}

Chapter partly based on:

Leoné, N.; Roy, M.; Saidi, S.; De Kort, G.; Hermida-Merino, D.;

Wilsens, C. H. R. M. ACS Omega 2019, 4 (6), 10376-10387.

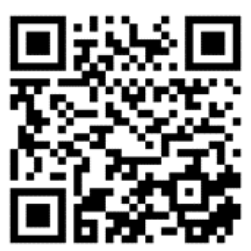

A big thanks goes to Nils Leone, and all co-authors for collaborations and discussions which helped us in finding the potential of the bis(2-oxazolines) precursor. 


\title{
In this chapter...
}
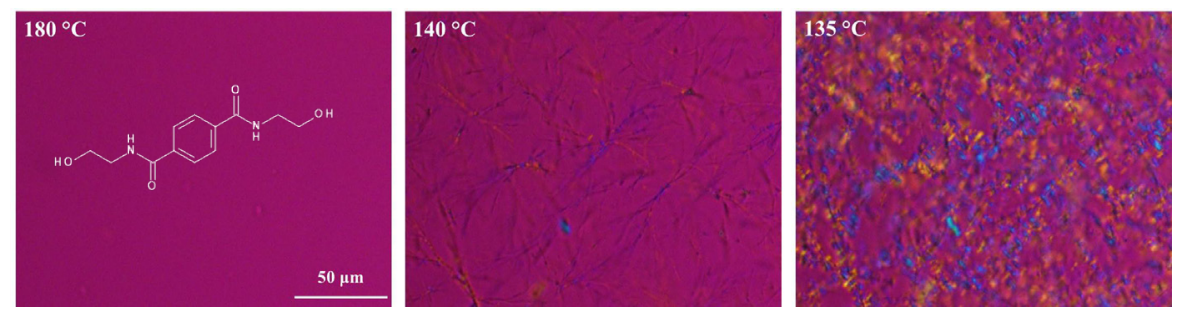

"Evaluating the performance of the precursor of bis(oxazolines) as an additive in a biopolymer (poly lactic acid)"

\begin{abstract}
After performing chemical recycling of polymers based on either terephthalic acid or 2,5-furandicarboxylic acid based 2-oxazolines (as is reported in chapters 1 and 2) the potential of these two organic compounds abbreviated as BHET and BHEF are evaluated as an additive for poly(lactic acid) (PLA) in this chapter. Interestingly, the combined plasticising and nucleating effect identifies BHET as a promising processing aid and nucleating agent for PLA.
\end{abstract}




\subsection{Introduction}

Poly-L-lactide (PLA) is an aliphatic thermoplastic polyester that can be derived through fermentation of renewable resources and has gained much attention as it is biodegradable when exposed to elevated temperatures and in the presence of bacteria $^{1,2}$. Despite its renewable origin, amorphous PLA generally suffers from brittle failure behavior which is generally related to its high glass transition temperature $\left(T_{\mathrm{g}}\right)$ around $60{ }^{\circ} \mathrm{C}$ and thus its high yield point ${ }^{3}$. Although PLA can be semi-crystalline with a melting temperature up to $180{ }^{\circ} \mathrm{C}$, it suffers from a slow crystal growth rate ${ }^{4}$. Furthermore, the melting and crystallization behavior of PLA is dependent on the presence of the D-enantiomer, as random incorporation of this enantiomer in the polymer backbone decreases the crystallinity, until a fully amorphous material is obtained ${ }^{5}$. With respect to processing, PLA is susceptible to hydrolysis, transesterification, and several degradation reactions at temperatures above $200{ }^{\circ} \mathrm{C}$, potentially decreasing the molecular weight and thus the mechanical performance $^{6}$. Despite these limitations, PLA has proven to be a promising material in, for example, packaging ${ }^{7}$, laminates ${ }^{8}$, composites $^{9,10}$ and 3D-printing ${ }^{11}$.

One route to counter the slow PLA crystal growth rate is through enhancement of the nucleation process: the introduction of a (foreign) organic or inorganic component can facilitate heterogeneous nucleation, thus acting as a nucleating agent (NA). One particularly interesting class of NAs includes meltsoluble hydrogen bonding organic compounds ${ }^{12,13}$. These compounds are designed to dissolve in the polymer matrix during processing and crystallize during cooling to generate surface for heterogeneous nucleation of the polymer matrix. This approach has successfully been employed to develop nucleating agents for PLA ${ }^{14-19}$, polyhydroxybutyrate ${ }^{20-22}$, isotactic polypropylene $(i \mathrm{PP})^{23-27}$, and poly $(1,4$-butylene adipate $)^{28}$.

In previous chapters we have elaborated on the performance of 2-oxazoline based polymers, together with their potential for hydrolysis and the consecutive isolation of the bispyrrolidone based monomers. So far, the role of the residual diol of the ring-opened 2-oxazoline has been ignored. However, the presence of two hydroxyl groups combined with two amide moieties makes them promising candidates for use as nucleating agents as they generally exhibit high melting temperatures and a strong ability to self-assemble/crystallize upon cooling. Accordingly, in this chapter we elaborate on the potential of bis(2hydroxyethyl)furan-2,5-dicarboxamide (BHEF) and N,N'-bis(2hydroxyethyl)terephthalamide (BHET), as additives for PLA (Figure 5.1). 

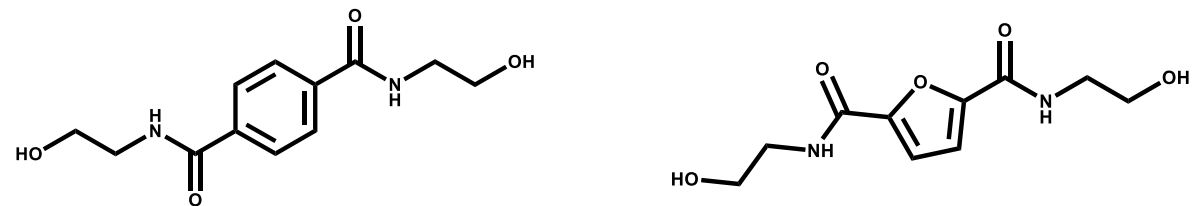

Figure 5.1. Chemical structure of the given organic compounds (left) N,N'-bis(2hydroxyethyl)terephthalamide (BHET) and (right) bis(2-hydroxyethyl)furan-2,5dicarboxamide (BHEF) used in this study.

\subsection{Experimental}

\subsubsection{Synthesis of $N, N^{\prime}-b i s(2-h y d r o x y e t h y l) t e r e p h t h a l a m i d e ~(B H E T)$ and BHEF}

The synthesis of N,N'-bis(2-hydroxyethyl)terephthalamide (henceforth abbreviated as BHET) was achieved through reaction of terephthalic acid $(10 \mathrm{~g}, 0.06$ $\mathrm{mol})$ and ethanol amine $(8 \mathrm{ml}, 0.132 \mathrm{~mol})$ in dimethylformamide at $150{ }^{\circ} \mathrm{C}$ for 18 hours. Next, the ensuing mixture was cooled and the formed crystallites were filtered, washed with methanol, and dried. The product was crystallized from methanol and dried overnight in vacuo at $60{ }^{\circ} \mathrm{C}$ prior to use. BHET was isolated with a yield of $78 \%$ and a purity $\geq 98 \%$, according to NMR analysis: ${ }^{1} \mathrm{H}-\mathrm{NMR}\left(\mathrm{CDCl}_{3}, 300 \mathrm{MHz}\right): \delta$ $8.55(\mathrm{~m}, 2 \mathrm{H}), 7.92(\mathrm{~s}, 2 \mathrm{H}), 4.74(\mathrm{~m}, 2 \mathrm{H}), 3.5(\mathrm{~m}, 4 \mathrm{H})$ and $3.35(\mathrm{~m}, 4 \mathrm{H})$. BHET did not display stable melting behavior; instead, melting is observed in polarized optical microscopy mounted with a Linkam Hotstage at $350{ }^{\circ} \mathrm{C}$, followed by immediate degradation.

Similarly, the synthesis of bis(2-hydroxyethyl)furan-2,5-dicarboxamide (named as BHEF henceforth) was achieved through reaction of furan dicarboxylic acid with ethanol amine, while using the similar reaction conditions as mentioned above. The product was crystallized from methanol and dried overnight in vacuo at $60{ }^{\circ} \mathrm{C}$ prior to use. BHEF was isolated with a yield of $67 \%$ and a purity $\geq 98 \%$, according to NMR analysis (provided in appendix D-figures D3 \& D4): ${ }^{1} \mathrm{H}-\mathrm{NMR}$ $\left(\mathrm{CDCl}_{3}, 300 \mathrm{MHz}\right): \delta 8.58(\mathrm{~m}, 2 * 1 \mathrm{H}), 7.12(\mathrm{~s}, 2 \mathrm{H}), 4.84(\mathrm{~m}, 2 * 1 \mathrm{H}), 3.51(\mathrm{~m}, 4 \mathrm{H})$ and $3.35(\mathrm{~m}, 4 \mathrm{H})$. BHEF displayed stable melting behavior at $150{ }^{\circ} \mathrm{C}$.

\subsubsection{Material Preparation and Blending Process}

PLA (L130 grade, Corbion) and additives (BHEF, HEABA and BHET) where dried for $24 \mathrm{~h}$ at $60{ }^{\circ} \mathrm{C}$ in vacuo prior to use. In this study, we extruded PLA samples with additives (BHET and BHEF), varying concentrations of $0 \mathrm{wt} \%$ (pure PLA), $0.5 \mathrm{wt} \%, 1.0 \mathrm{wt} \%, 1.5 \mathrm{wt} \%$ and $2.0 \mathrm{wt} \%$. All samples were mixed in the desired ratio prior to blending in a DSM Explore twin-screw micro-extruder with a 
barrel size of $5 \mathrm{ml}$. The samples were processed at $200{ }^{\circ} \mathrm{C}$ for three minutes $(100$ $\mathrm{rpm}$ ), under constant nitrogen flow. After processing, when needed the extrudate was either cooled till room temperature or directly injection molded into tensile bars (2 $\mathrm{mm} \times 4 \mathrm{~mm} \times 70 \mathrm{~mm}$, with a gage length of $25 \mathrm{~mm}$ ) using a DSM Xplore IM 5.5 micro injection mold with a barrel temperature of $200^{\circ} \mathrm{C}$ and a mold temperature of $25^{\circ} \mathrm{C}$.

\subsubsection{Characterization Methods}

${ }^{1} \mathrm{H}-\mathrm{NMR}$ spectra were recorded with a Bruker Ultrashield 300 spectrometer (300 MHz magnetic field). NMR-samples were prepared by dissolving ca. $10 \mathrm{mg}$ of sample in $0.5 \mathrm{ml}$ deuterated chloroform $\left(\mathrm{CDCl}_{3}\right)$. Spectra were referenced against tetramethyl silane (TMS).

Weight average molecular weight $\left(M_{\mathrm{w}}\right)$ and polydispersity (PDI) of PLA blends after processing were determined from gel permeation chromatography (GPC) on a PSS SECcurity GPC system using Agilent 1260 Infinity instrument technology. The GPC system is equipped with two PFG combination medium micro-columns with $7 \mu \mathrm{m}$ particle size (4.6 x $250 \mathrm{~mm}$, separation range 100-1.000.000 Da), a PFG combination medium pre-column with $7 \mu \mathrm{m}$ particle size $(4.6 \times 30 \mathrm{~mm})$, and a Refractive Index detector (RI). Distilled 1,1,1,3,3,3-hexafluoroisopropanol (HFIP) containing $0.019 \%$ sodium trifluoroacetate was used as mobile phase at $40{ }^{\circ} \mathrm{C}$, with a $0.3 \mathrm{~mL} / \mathrm{min}$ flow rate. The GPC apparatus was calibrated with poly(methyl methacrylate) standards obtained from PSS. GPC samples were prepared by dissolving $5 \mathrm{mg}$ of PLA in $1.5 \mathrm{ml}$ HFIP overnight, after which the samples were filtered over a $0.2 \mu \mathrm{m}$ PTFE syringe filter prior to injection.

Differential scanning calorimetry (DSC) was performed on the samples using a TA Instruments Q2000 DSC. All samples were measured at heating and cooling rates of $1,3,10,30$, or $100{ }^{\circ} \mathrm{C} / \mathrm{min}$ up to a maximum temperature of $200{ }^{\circ} \mathrm{C}$, unless mentioned otherwise. The glass transition temperature $\left(T_{\mathrm{g}}\right)$ and peak melting temperature $\left(T_{\mathrm{m}}\right)$ were determined from heating runs. The crystallization exotherm observed during the first cooling run was used to define peak crystallization temperature $\left(T_{\mathrm{c}}\right)$ and onset of crystallization temperature $\left(T_{\text {ons }}\right)$. The peak crystallization temperature is considered the peak value of the crystallization exotherm, whereas the intersection of the tangents of the baseline and the crystallization curve is considered to be the onset temperature for crystallization.

Polarization optical micrographs were taken (between cross-polarizers and using a $530 \mathrm{~nm} \lambda$-wave plate) on an Olympus BX53 microscope mounted with an Olympus DP26 camera and a Linkam hotstage. The samples were heated to the desired temperature (generally $200{ }^{\circ} \mathrm{C}$ ) at a rate of $30{ }^{\circ} \mathrm{C} / \mathrm{min}$. After leaving the 
samples for $3 \mathrm{~min}$ under isothermal conditions, the samples were cooled at a specified cooling rate (generally being 3,10 , or $30^{\circ} \mathrm{C} / \mathrm{min}$ ) to the desired temperature where crystallization was monitored for 30 minutes. Optical micrographs depicting the morphological changes were recorded both during cooling and under isothermal conditions.

The evolution of the complex viscosity of the PLA blends was followed over time at $200{ }^{\circ} \mathrm{C}$ in an Anton Paar 702 twin drive rheometer. Parallel plate geometry, having a diameter of $15 \mathrm{~mm}$ and gap of $1000 \mu \mathrm{m}$, was used to study the time dependence of the complex viscosity. The strain (1\%) and angular frequency (10 $\mathrm{rad} / \mathrm{s}$ ) were chosen such that the experiments were performed in the linear viscoelastic regime.

\subsection{Results and discussion}

\subsubsection{Influence of processing conditions on molecular weight of PLA}

In addition to the thermal degradation of PLA during processing ${ }^{1,2}$, the presence of water or alcohol groups is known to facilitate hydrolysis or transesterification, effectively lowering the molecular weight of the polymer. In turn, such a molecular weight decrease can result in the suppression in mechanical performance of the final product. Given that BHET and BHET both bear two hydroxyl groups, we evaluated the rate of transesterification with PLA upon processing prior to evaluation of their potential as an additive. Accordingly, various concentrations of BHET and BHEF $(0.5-2.0 \mathrm{wt} \%)$ were extruded with PLA for three minutes at $200{ }^{\circ} \mathrm{C}$ and the resulting blends were subjected to GPC analysis. As can be observed from the GPC traces shown in Figure 5.2 and the molecular weights shown in Table 5.1, both BHET and BHEF facilitate a slight suppression in $M_{\mathrm{w}}$ during processing; The pure PLA sample shows a $M_{\mathrm{w}}$ of $138 \mathrm{~kg} / \mathrm{mol}$, whereas the presence of $2.0 \mathrm{wt} \%$ of BHET yields PLA with a $M_{\mathrm{w}}$ of $129 \mathrm{~kg} / \mathrm{mol}$. Similarly, the presence of BHEF yields PLA with a $M_{\mathrm{w}}$ of $117 \mathrm{~kg} / \mathrm{mol}$. 
Table 5.1. Weight average molecualr weigh $\left(M_{w}\right)$ and polydispersity index (PDI) of the PLA after processing with various concentration BHET and BHEF, according to HFIP-GPC analy sis referenced against PMMA standards.

\begin{tabular}{|ccc|}
\hline Sample & $\boldsymbol{M}_{\mathrm{w}}(\mathrm{g} / \mathrm{mol})$ & PDI (-) \\
\hline PLA pure & 138.000 & 2.16 \\
\hline PLA + 0.5 wt\% BHET & 134.000 & 2.17 \\
\hline PLA + 1.0 wt\% BHET & 138.000 & 2.18 \\
\hline PLA + 1.5 wt\% BHET & 130.000 & 2.13 \\
\hline PLA + 2.0 wt\% BHET & 129.000 & 2.11 \\
\hline PLA + 0.5 wt\% BHEF & 131.000 & 1.95 \\
\hline PLA + 1.0 wt\% BHEF & 127.000 & 1.96 \\
\hline PLA + 1.5 wt\% BHEF & 121.000 & 2.01 \\
\hline PLA + 2.0 wt\% BHEF & 117.000 & 1.94 \\
\hline
\end{tabular}

It appears that transesterification proceeds slightly faster in with BHEF compared to BHET. A possible explanation for this behavior is found in the difference in melting temperature of the BHEF $\left(150{ }^{\circ} \mathrm{C}\right)$ and BHET $\left(>300{ }^{\circ} \mathrm{C}\right)$; BHET needs to solubilize first in the PLA matrix during extrusion before it can participate in the transesterification reaction. In contrast, the processing temperature $\left(200{ }^{\circ} \mathrm{C}\right)$ is well above the melting temperature of BHEF, allowing for transesterification throughout the whole extrusion. Nevertheless, though a molecular weight decrease is observed in the presence of BHEF (15\% decrease for $2.0 \mathrm{wt} \%$ sample) and BHET (7\% decrease for $2.0 \mathrm{wt} \%$ sample), we do not expect the transesterification significant enough to affect the mechanical properties of the PLA since the residual molecular weights are well above the entanglement molecular weight of PLA. 
A)

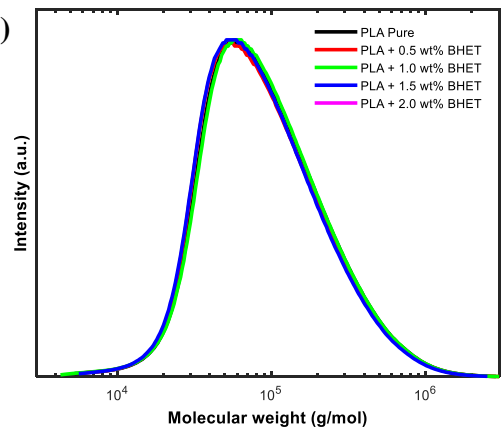

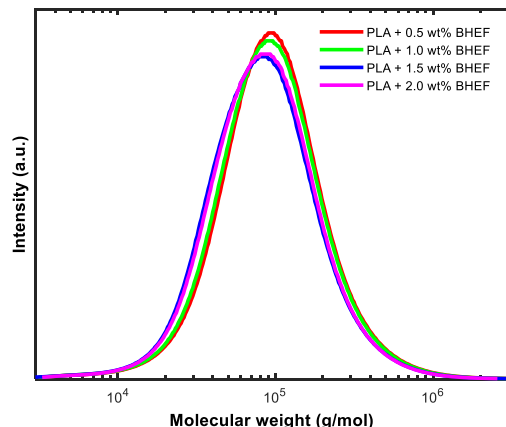

Molecular weight $(\mathrm{g} / \mathrm{mol})$

Figure 5.2. GPC traces of the PLA after processing with various concentration of BHET (left) and BHEF (right).

To identify how the diols affect the visco-elastic behavior of the PLA matrix during prolonged heating, we have loaded the BHET samples in the rheometer (plateplate geometry) at $200{ }^{\circ} \mathrm{C}$ and monitored the evolution of the complex viscosity over time (Figure 5.3A). Note, only BHET was used for this experiment as BHEF proves to trans esterify significantly faster with the PLA as observed from GPC analysis. Therefore, only determination of the complex viscosity of samples with BHEF was performed at a frequency of $10 \mathrm{rad} / \mathrm{s}$ without subjecting the samples to a time-sweep. Note, in the protocol used for the generation of the data displayed in Figure 5.3A, we start to monitor the exposure time directly after loading of the sample, though the equilibration time is not considered in the time sweeps. Generally, we observe that the pure PLA is stable under the evaluated conditions, indicated by the rather constant complex viscosity over time. As expected, the complex viscosity of PLA in the presence of BHET decreases over time. Furthermore, the complex viscosity decreases more rapidly over time as the BHET concentration increases. Given the increasing concentration of hydroxyl groups in the system, we consider it plausible that this decrease in complex viscosity is the result of the transesterification reaction of BHET with the PLA matrix. This decrease in $M_{\mathrm{w}}$ is rather significant; after roughly 3000 seconds the molecular weight of the PLA has decreased with roughly $60 \%$ in the presence of $2 \mathrm{wt} \%$ BHET compared to pure PLA, indicated by the factor 20 difference in complex viscosity between the samples (and the use of the relation $\eta_{0} \sim$ $M_{\mathrm{w}}{ }^{3.4}$ for linear entangled polymer chains). 

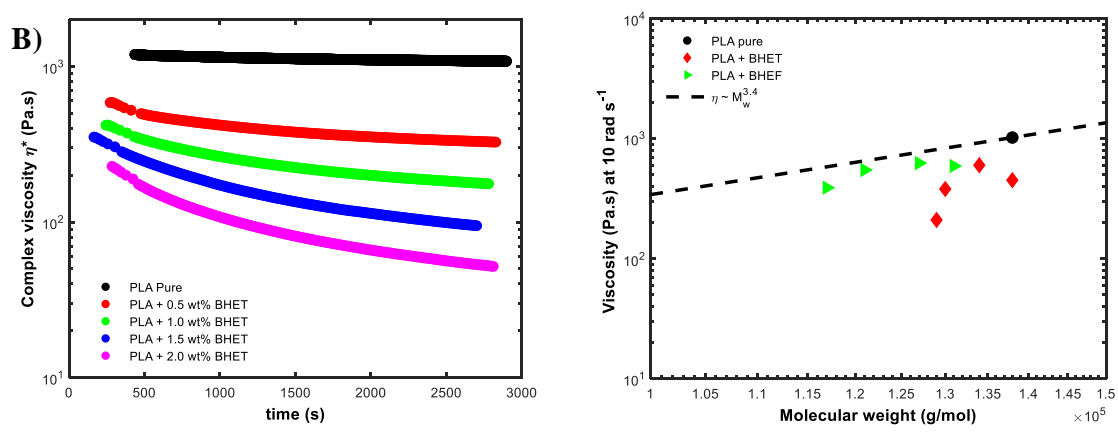

Figure 5.3. A) GPC traces of the PLA after processing with various concentration of BHET. B) Dependency of the complex viscosity over time for PLA with various concentration of BHET.

Interestingly, when looking closely at the complex viscosity values (10 $\mathrm{rad} / \mathrm{s}$ ), being in the terminal regime) at the start of the experiment we can observe that BHEF does follow the expected trend based on the relation $\eta_{0} \sim M_{\mathrm{w}}{ }^{3.4}$ (Figure 5.3), confirming that the viscosity decrease is solely the result of transesterification. In contrast, the BHET does not seem to follow the expected trend; instead the observed complex viscosity appears lower than expected. This would suggest that BHET acts as a plasticizer for PLA, effectively lowering the viscosity of the melt in addition to the viscosity suppression resulting from the transesterification process.

\subsubsection{BHEF and BHET as nucleating agents for PLA}

From the previous section we can conclude that BHET and BHEF can readily be used as additives for PLA under processing conditions, although continued exposure to elevated temperatures should be minimized to limit transesterification. To evaluate how the BHET and BHEF affect the nucleating efficiency of PLA, the samples were subjected to DSC analysis. Note, the sample having $2.0 \mathrm{wt} \%$ of BHET required heating to temperatures above $220{ }^{\circ} \mathrm{C}$ under quiescent conditions to fully melt the BHET crystallites. Such a high temperature is likely to accelerate both chain degradation and transesterification of PLA by BHET molecules. Therefore, samples containing $2.0 \mathrm{wt} \%$ BHET are excluded from DSC experiments. For the other samples, the DSC traces are shown in Figure 5.4. 

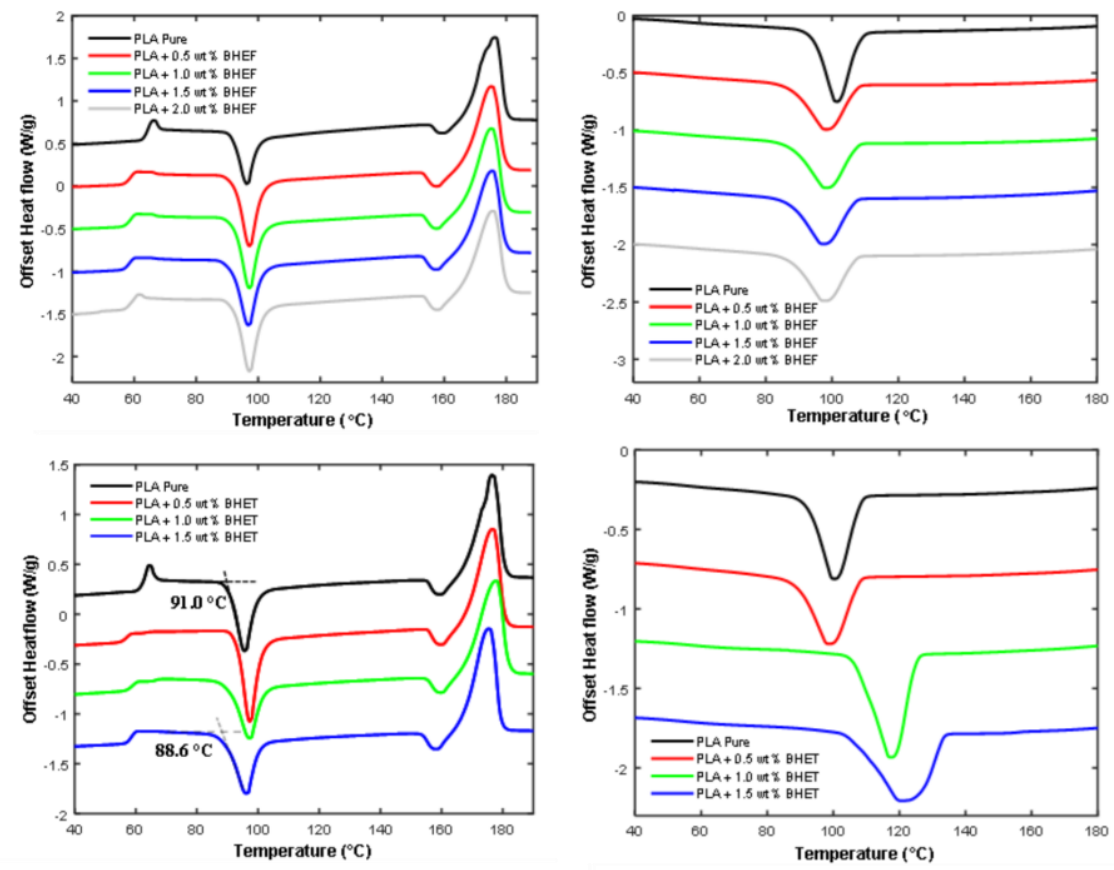

Figure 5.4. Differential scanning calorimetry traces of PLA samples having varying concentration of BHEF (top) and BHET (bottom). First DSC heating traces are decpicted on the left, whereas the crystallization exotherms observed during the first cooling trace are depicted on the right (heating and cooling performed at a rate of $\left.10^{\circ} \mathrm{C}\right)$.

During the first heating, the pure PLA exhibits a distinct glass transition temperature $\left(T_{\mathrm{g}}\right)$ around $64^{\circ} \mathrm{C}$. We observe a slight suppression in the $T_{\mathrm{g}}$ to roughly $58{ }^{\circ} \mathrm{C}$ in the presence of BHEF and, whereas PLA samples with BHET exhibit a decreased $T_{\mathrm{g}}$ between 56 and $59{ }^{\circ} \mathrm{C}$. Upon further heating, cold crystallization of the samples is observed with a peak value around $97{ }^{\circ} \mathrm{C}$ for both BHEF and BHET. A small exotherm is observed around $160{ }^{\circ} \mathrm{C}$, which is thought to result from the rearrangement of disordered $\alpha^{\prime}$ crystals formed during cold-crystallization into the thermodynamically stable $\alpha$ crystallites $^{29,30}$. Further heating eventually results in melting of the $\alpha$-crystallites as is evident from the melting endotherms at $175-177^{\circ} \mathrm{C}$ depicted in Figures 5.4, left.

After melting of the PLA crystallites, all samples are cooled to $40{ }^{\circ} \mathrm{C}$ at a rate of $10^{\circ} \mathrm{C} / \mathrm{min}$. During cooling (Figure 5.4, right), crystallization of the pure PLA is observed with an onset of crystallization ( $\left.T_{\mathrm{c} \_o n s}\right)$ at $108.1^{\circ} \mathrm{C}$ and an enthalpy of crystallization $\left(\Delta H_{\mathrm{c}}\right)$ of $34.0 \mathrm{~J} / \mathrm{g}$. Interestingly, the presence of BHEF does not seem 
to significantly influence the crystallization of PLA (provided in appendix D-figure D1 \& D5); the $T_{\mathrm{c} \_ \text {ons }}$ is observed around $107-108{ }^{\circ} \mathrm{C}$, while the $\Delta H_{\mathrm{c}}$ increases slightly to $40-41 \mathrm{~J} / \mathrm{g}$. It is expected that this slight increase in crystallinity could be the result of the lowered molecular weight, which generally results in a better crystallization. In contrast, BHET crystallization is delayed slightly in the presence of $0.5 \mathrm{wt} \%$ and starts at $107.8{ }^{\circ} \mathrm{C}\left(\Delta H_{\mathrm{c}}=30.7 \mathrm{~J} / \mathrm{g}\right)$, potentially resulting from the plasticizing effect of BHET ${ }^{5}$. Increasing the concentration of BHET to 1 and $1.5 \mathrm{wt} \%$ increases the onset of crystallization to $125{ }^{\circ} \mathrm{C} \quad\left(\Delta H_{\mathrm{c}}=46.3 \mathrm{~J} / \mathrm{g}\right)$ and $133^{\circ} \mathrm{C} \quad\left(\Delta H_{\mathrm{c}}=48.3 \mathrm{~J} / \mathrm{g}\right)$, respectively. The increase in onset of crystallization can be converted to the nucleation efficiency using the equation $\mathrm{NE}=\left(\left(T_{\mathrm{c}}-T_{\mathrm{c}}{ }^{\min }\right) /\left(T_{\mathrm{c}}{ }^{\mathrm{max}}-T_{\mathrm{c}}{ }^{\mathrm{min}}\right)\right) \cdot 100 \%$ described by Lotz and coworkers ${ }^{31}$, where $T_{\mathrm{c}}$ is the peak crystallization temperature, $T_{\mathrm{c}}{ }^{\max }$ is the maximum crystallization peak temperature of self-nucleated PLA (156.3 ${ }^{\circ} \mathrm{C}$ for this PLA grade ${ }^{32}$ ), and $T_{\mathrm{c}}{ }^{\mathrm{min}}$ is the minimum crystallization peak temperature occurring in the absence of heterogeneous nucleation sites, hence as it proceeds in pure PLA. Indeed, one can observe that the use of $0.5 \mathrm{wt} \%$ BHET results in a negative nucleating efficiency (Table 5.2), confirming that the BHET delays the nucleation process of PLA when it resides in the dissolved state. Upon increasing the BHET concentration, the NE increases to values above 30\%. An overview of the thermal transitions of the samples with BHET is provided in Table 5.2, while for BHEF is shown in Table 5.3.

To reflect, the nucleating efficiency of BHET is higher than that of Talc (NE $=28.7 \%$ ), but lower compared to other melt-soluble oxalamide or hydrazide based nucleating agents $(\mathrm{NE}=49.7 \text { and 53.6, respectively })^{32}$. In contrast, the $T_{\mathrm{c}}$ for the samples containing BHEF are between 97 and $99{ }^{\circ} \mathrm{C}$, translating to a NE of -2.5 to $5 \%$, supporting the notion that BHEF does not act as nucleating agent for PLA. To identify the origin of the enhancement in the nucleation process of PLA in the presence of BHET, polarized optical microscopy experiments mounted with a Linkam Hotstage have been conducted samples containing various BHET concentration. As can be seen from Figure 5.5, the enhancement in nucleating ability of PLA in the presence of $1.0 \mathrm{wt} \%$ BHET has its origin in the melt-soluble nature of BHET; As is shown in Figure 5.5, left, upon heating the sample to $180{ }^{\circ} \mathrm{C}$, a homogenous mixture is obtained. However, upon cooling at a rate of $10{ }^{\circ} \mathrm{C} / \mathrm{min}$, the growth of tree-like BHET crystal is observed, which, upon further cooling, provide surface for heterogeneous nucleation of the PLA. 
Table 5.2. Characterstic thermal transitions observed during the first heating and cooling runs in DSC analysis on the PLA samples having varying concentration of BHET.

\begin{tabular}{|c|c|c|c|c|c|c|c|c|c|c|}
\hline \multirow[b]{2}{*}{$\begin{array}{c}\text { Sample } \\
\text { name }\end{array}$} & \multicolumn{6}{|c|}{ First heating $\left(10^{\circ} \mathrm{C} / \mathrm{min}\right)$} & \multicolumn{4}{|c|}{ First cooling $\left(10^{\circ} \mathrm{C} / \mathrm{min}\right)$} \\
\hline & $\begin{array}{c}T_{\mathrm{g}} \\
\left({ }^{\circ} \mathrm{C}\right)\end{array}$ & $\begin{array}{c}T_{c c_{-} \text {ons }}{ }^{a} \\
\left({ }^{\circ} \mathbf{C}\right)\end{array}$ & $\begin{array}{l}T_{\mathrm{cc}}^{\mathrm{a}} \\
\left({ }^{\circ} \mathrm{C}\right)\end{array}$ & $\begin{array}{l}\Delta H_{\mathrm{cc}} \\
(\mathrm{J} / \mathrm{g})\end{array}$ & $\begin{array}{c}T_{\mathrm{m}} \\
\left({ }^{\circ} \mathbf{C}\right)\end{array}$ & $\begin{array}{l}\Delta H_{\mathrm{m}} \\
(\mathrm{J} / \mathrm{g})\end{array}$ & $\begin{array}{c}T_{c_{-} \text {ons }} b \\
\left({ }^{\circ} \mathrm{C}\right)\end{array}$ & $\begin{array}{c}T_{\mathrm{c}}^{\mathrm{b}} \\
\left({ }^{\circ} \mathrm{C}\right)\end{array}$ & $\begin{array}{l}\Delta H_{\mathrm{c}} \\
(\mathrm{J} / \mathrm{g})\end{array}$ & $\begin{array}{l}N E \\
(\%)\end{array}$ \\
\hline PLA pure & 63.5 & 91.0 & 95.6 & 29.2 & 176.5 & 44.9 & 108.1 & 100.2 & 34.0 & 0 \\
\hline $\begin{array}{c}\text { PLA + 0.5 } \\
\text { BHET }\end{array}$ & $\begin{array}{c}56.7 / \\
63.6\end{array}$ & 93.1 & 97.3 & 32.0 & 176.6 & 45.8 & 107.8 & 98.8 & 30.7 & -2 \\
\hline $\begin{array}{c}\text { PLA + 1.0 } \\
\text { BHET }\end{array}$ & $\begin{array}{c}58.2 / \\
65.4\end{array}$ & 90.8 & 97.1 & 28.8 & 177.6 & 46.7 & 124.9 & 117.6 & 46.3 & 31 \\
\hline $\begin{array}{c}\text { PLA + } 1.5 \\
\text { BHET }\end{array}$ & $\begin{array}{c}58.5 / \\
65.3\end{array}$ & 88.6 & 96.1 & 29.8 & 175.4 & 45.9 & 133.5 & 120.5 & 48.3 & 36 \\
\hline
\end{tabular}

${ }^{a} T_{c c_{-} o n s}$ denoted the onset temperature for cold-crystallization, $T_{c c}$ denotes the peak value of the cold-crystallization exotherm. ${ }^{b} T_{c_{-} o n s}$ denotes the onset temperature for crystallization during cooling, whereas $T_{c}$ denotes the peak value of the crystallization exotherm. ${ }^{c} N E$ reflects the nucleating efficiency, calculated using the equation $N E=\left(\left(T_{c}-T_{c}{ }^{\min }\right) /\left(T_{c}{ }^{\max }-T_{c}{ }^{\min }\right)\right) \cdot 100 \%$.

When performing the same experiment in the presence of only $0.5 \mathrm{wt} \%$ BHET, no BHET crystal formation is observed prior to the homogenous crystallization of the PLA itself, in line with observations from DSC analysis. This would suggest that the BHET concentration is too low to crystallize from the PLA melt upon cooling and hence it only facilitates a plasticizing effect. Overall, this indicates that BHET is indeed melt-soluble nucleating agent for PLA, though only when in a concentration of $1.0 \mathrm{wt} \%$ and higher. 
Table 5.3. Characterstic thermal transitions observed during the first heating and cooling runs in DSC analysis on the PLA samples having varying concentration of BHEF.

\begin{tabular}{|c|c|c|c|c|c|c|c|c|c|c|}
\hline \multirow[b]{2}{*}{$\begin{array}{c}\text { Sample } \\
\text { name }\end{array}$} & \multicolumn{6}{|c|}{ First heating $\left(10^{\circ} \mathrm{C} / \mathrm{min}\right)$} & \multicolumn{4}{|c|}{ First cooling $\left(10^{\circ} \mathrm{C} / \mathrm{min}\right)$} \\
\hline & $\begin{array}{c}T_{\mathrm{g}} \\
\left({ }^{\circ} \mathbf{C}\right)\end{array}$ & $\begin{array}{c}T_{c c \_o n s}{ }^{a} \\
\left({ }^{\circ} \mathrm{C}\right)\end{array}$ & $\begin{array}{l}T_{\mathrm{cc}^{\mathrm{a}}} \\
\left({ }^{\circ} \mathrm{C}\right)\end{array}$ & $\begin{array}{l}\Delta H_{\mathrm{cc}} \\
(\mathrm{J} / \mathrm{g})\end{array}$ & $\begin{array}{c}T_{\mathrm{m}} \\
\left({ }^{\circ} \mathrm{C}\right)\end{array}$ & $\begin{array}{l}\Delta \boldsymbol{H}_{\mathrm{m}} \\
(\mathrm{J} / \mathrm{g})\end{array}$ & $\begin{array}{c}T_{c_{-} \text {ons }}{ }^{b} \\
\left({ }^{\circ} \mathrm{C}\right)\end{array}$ & $\begin{array}{c}T_{\mathrm{c}}^{\mathrm{b}} \\
\left({ }^{\circ} \mathrm{C}\right)\end{array}$ & $\begin{array}{l}\Delta \boldsymbol{H}_{\mathrm{c}} \\
(\mathrm{J} / \mathbf{g})\end{array}$ & $\begin{array}{l}N E \\
(\%)\end{array}$ \\
\hline PLA pure & 63.5 & 91.0 & 95.6 & 29.2 & 176.5 & 44.9 & 108.1 & 100.2 & 34.0 & 0 \\
\hline $\begin{array}{c}\text { PLA + 1.0 } \\
\text { BHEF }\end{array}$ & 59.1 & 90.75 & 97.03 & 32.39 & 175.07 & 46.24 & 107.3 & 97.9 & 39.2 & $\begin{array}{c}- \\
4.1\end{array}$ \\
\hline $\begin{array}{c}\text { PLA + 1.5 } \\
\text { BHEF }\end{array}$ & 58.6 & 90.13 & 96.6 & 33.93 & 175.3 & 46.4 & 106.4 & 96.9 & 39.0 & $\begin{array}{l}- \\
5.9\end{array}$ \\
\hline $\begin{array}{c}\text { PLA + 2.0 } \\
\text { BHEF }\end{array}$ & 58.8 & 90.67 & 97.2 & 33.59 & 175.84 & 46.2 & 106.8 & 97.33 & 31.5 & $\begin{array}{l}- \\
5.1\end{array}$ \\
\hline
\end{tabular}

${ }^{a} T_{c c_{-} \text {ons }}$ denoted the onset temperature for cold-crystallization, $T_{c c}$ denotes the peak value of the cold-crystallization exotherm. ${ }^{b} T_{c_{-} \text {ons }}$ denotes the onset temperature for crystallization during cooling, whereas $T_{c}$ denotes the peak value of the crystallization exotherm. ${ }^{c} N E$ reflects the nucleating efficiency, calculated using the equation $N E=\left(\left(T_{c}-T_{c}{ }^{{ }^{i n}}\right) /\left(T_{c}{ }^{{ }^{m a x}}-T_{c}{ }^{\text {min }}\right)\right) \cdot 100 \%$.

In contrast, no BHEF crystallization is observed prior to the homogeneous nucleation of PLA, irrespective of the employed BHEF concentration. This likely explains the inability of BHEF to enhance the nucleation process of PLA; the low melting temperature of BHEF prevents its crystallization from the PLA solution at temperatures where heterogeneous nucleation of PLA can occur. Instead, homogenous nucleation of PLA is observed for all samples containing BHEF. Given that BHEF does not seem to hold any plasticizing or nucleating capability for PLA, we will only proceed with samples containing BHET in the remainder of the paper. 

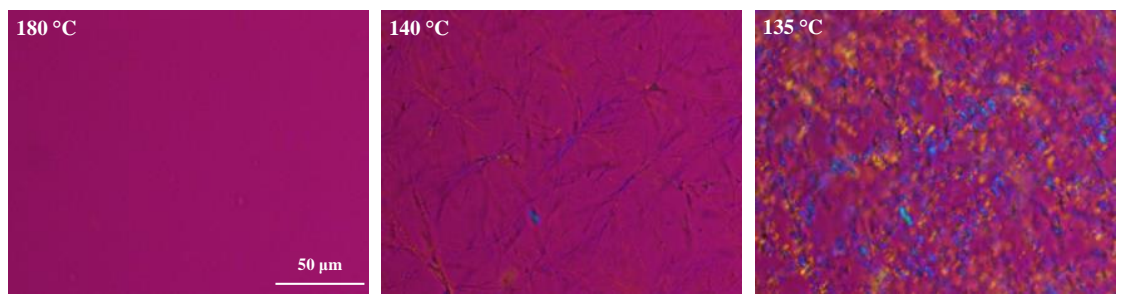

Figure 5.5. Optical micrographs recorded between crossed polars and $530 \mathrm{~nm} \lambda$ wave plate of the sample having $1.0 \mathrm{wt} \%$ of BHET. Left) homogenous solution of BHET in PLA, middle) crystallization of treelike BHET crystallites from the PLA melt, right) PLA crystals growing on the surface of the BHET crystallites. Images were taken during cooling at a rate of $10^{\circ} \mathrm{C} / \mathrm{min}$, after heating the sample to $200{ }^{\circ} \mathrm{C}$.

\subsubsection{Effect of cooling rate on BHET morphology and nucleating efficiency of PLA}

As is observed from Figure 5.5, the crystallization of BHET from the PLA melt itself proceeds via a nucleation and growth, a process that is driven by the supersaturation; By decreasing the temperature of the polymer melt containing dissolved BHET, one decreases the solubility until a supersaturated state is achieved, a process that is dependent on both the temperature and the NA concentration. In line with observations from Figure 5.4, crystallization of a compound from such a supersaturated state effectively yields a homogenous dispersion of BHET crystals throughout the polymer matrix. The overall nucleating efficiency of the polymer matrix is determined by the geometry and the surface-to-volume ratio of the NA crystals, which in turn is dependent on the thermal history of the sample, and hence the supersaturation of the system prior to the NA crystallization. Therefore, we expect that the morphology of the BHET crystallites generated during cooling will strongly depend on both the thermal history and BHET concentration. To evaluate the effect of cooling rate on the BHET crystal morphology generated during cooling and its resulting effect on the nucleation of PLA, we have heated the samples to $200{ }^{\circ} \mathrm{C}$, cooled them to $140{ }^{\circ} \mathrm{C}$ at a rate of $1,3,10,30$, or $100{ }^{\circ} \mathrm{C} / \mathrm{min}$, and monitored the crystallization process under isothermal conditions. These experiments have been performed both in a polarized optical microscpe mounted with a Linkam Hotstage and in DSC analysis. Figure 5.6 displays the characterstic BHET morphology and the ensuing PLA crystallization for pure PLA and for PLA with $1.5 \mathrm{wt} \%$ BHET, as observed in polarized optical microscopy. 
In general, we observe that the nucleation density in PLA during isothermal crystallization at $140{ }^{\circ} \mathrm{C}$ is very low, which, combined with the slow crystal growth rate, results in the growth of large PLA spherulites over time (top-right image in Figure 5.6). In contrast, the formation of 'treelike' BHET crystallites is detected around $170{ }^{\circ} \mathrm{C}$ when subjecting PLA with $1.5 \mathrm{wt} \%$ BHET to the same experimental protocol. PLA crystal growth proceeds rapidly on the surface of the BHET network when reaching $140{ }^{\circ} \mathrm{C}$, effectively covering the whole view area in microscopy with crystallites within 60 seconds. Upon subjecting the PLA with $1.5 \mathrm{wt} \%$ BHET to decreasing cooling rates, we observe a change in the BHET crystal morphology/habit. Cooling from $200{ }^{\circ} \mathrm{C}$ to $140{ }^{\circ} \mathrm{C}$ at a rate of $10{ }^{\circ} \mathrm{C} / \mathrm{min}$ favors the growth of thick and short BHET fibrils, which becomes even more pronounced during cooling at a rate of $3{ }^{\circ} \mathrm{C} / \mathrm{min}$. In other words, the surface-to-volume ratio of the BHET crystallites decreases dramatically when they are allowed to crystallize more slowly. Such a decrease in available surface for nucleation of the PLA matrix decreases the nucleation efficiency, as is clearly observed in the optical micrographs taken during isothermal crystallization at $140{ }^{\circ} \mathrm{C}$. 

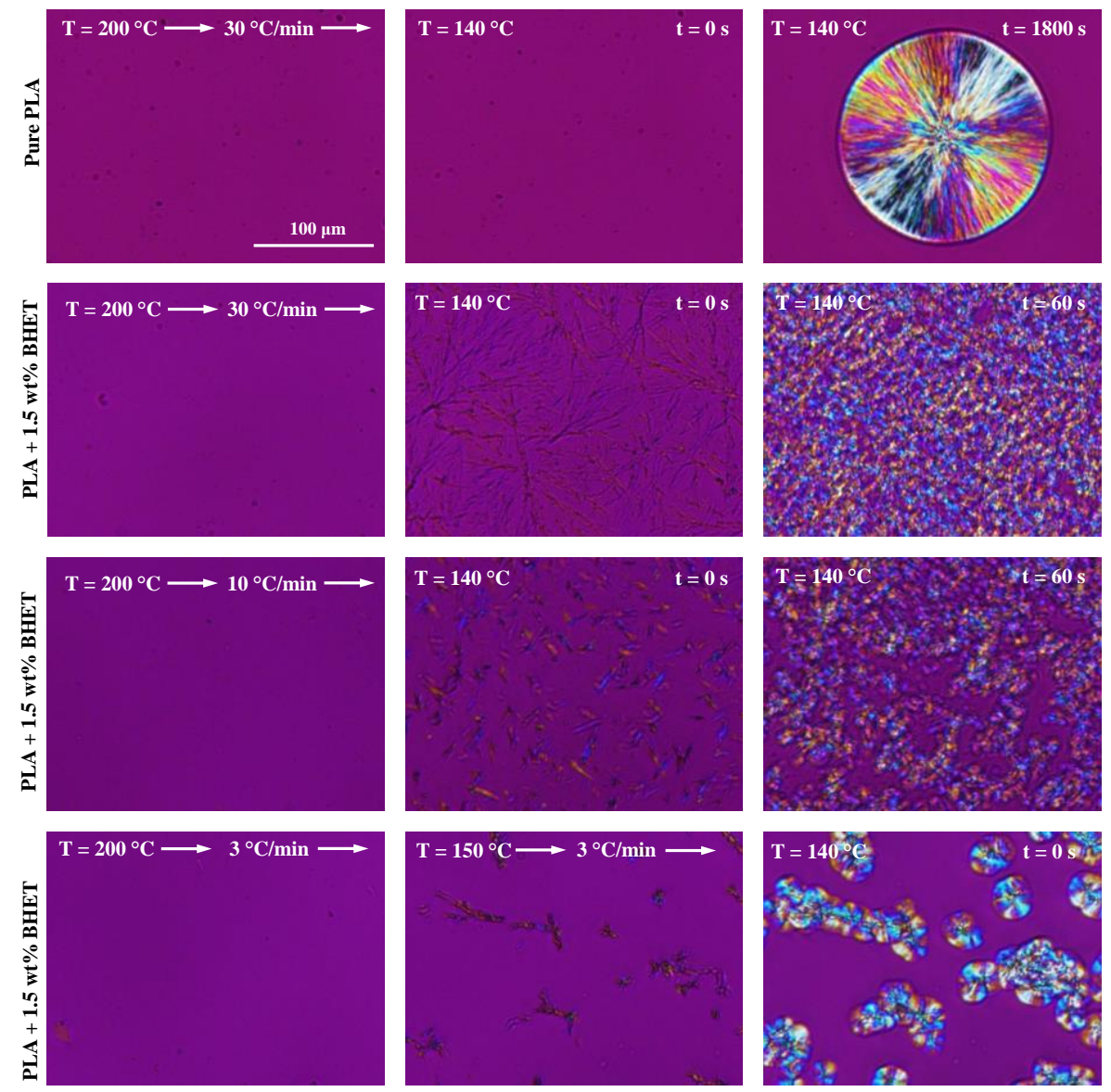

Figure 5.6. Optical micrographs taken between crossed polars and a $530 \mathrm{~nm} \lambda$-wave plate of pure PLA and PLA +1.5 wt\% BHET. The samples were first heated to 200 ${ }^{\circ} \mathrm{C}$ and cooled at a specific rate of 3,10 , or $30{ }^{\circ} \mathrm{C} / \mathrm{min}$ to $140{ }^{\circ} \mathrm{C}$ after which the sample was allowed to crystallize under isothermal conditions. Note, For the sample cooled at $3{ }^{\circ} \mathrm{C} / \mathrm{min}$, nucleation proceeds already upon cooling to $140{ }^{\circ} \mathrm{C}$ due to the low cooling rate and the presence of the BHET crystallites. The scale bar, provided in the top left image, is the same for all micrographs.

The same features are observed from DSC analysis during isothermal crystallization in of the samples at $140{ }^{\circ} \mathrm{C}$ after cooling them at various rates from $200{ }^{\circ} \mathrm{C}$ to $140{ }^{\circ} \mathrm{C}$; As is evident from Figure 5.7 for samples containing 1.0 and 1.5 wt $\%$ BHET, the higher the applied cooling rate, the faster the increase in crystalline fraction of PLA during isothermal crystallization at $140{ }^{\circ} \mathrm{C}$. This behavior is 
undoubtedly resulting from the increased surface-to-volume ratio of the BHET crystallites and thus the enhanced nucleation density of PLA. Furthermore, upon comparison of the isothermal crystallization endotherms at the same cooling rate, we observe that the sample containing $1.5 \mathrm{wt} \%$ BHET crystallizes faster than the sample containing $1.0 \mathrm{wt} \%$ BHET. Again, we expect that this is the result of the higher volume fraction of BHET crystals, effectively increasing the surface available for heterogenous nucleation of PLA.
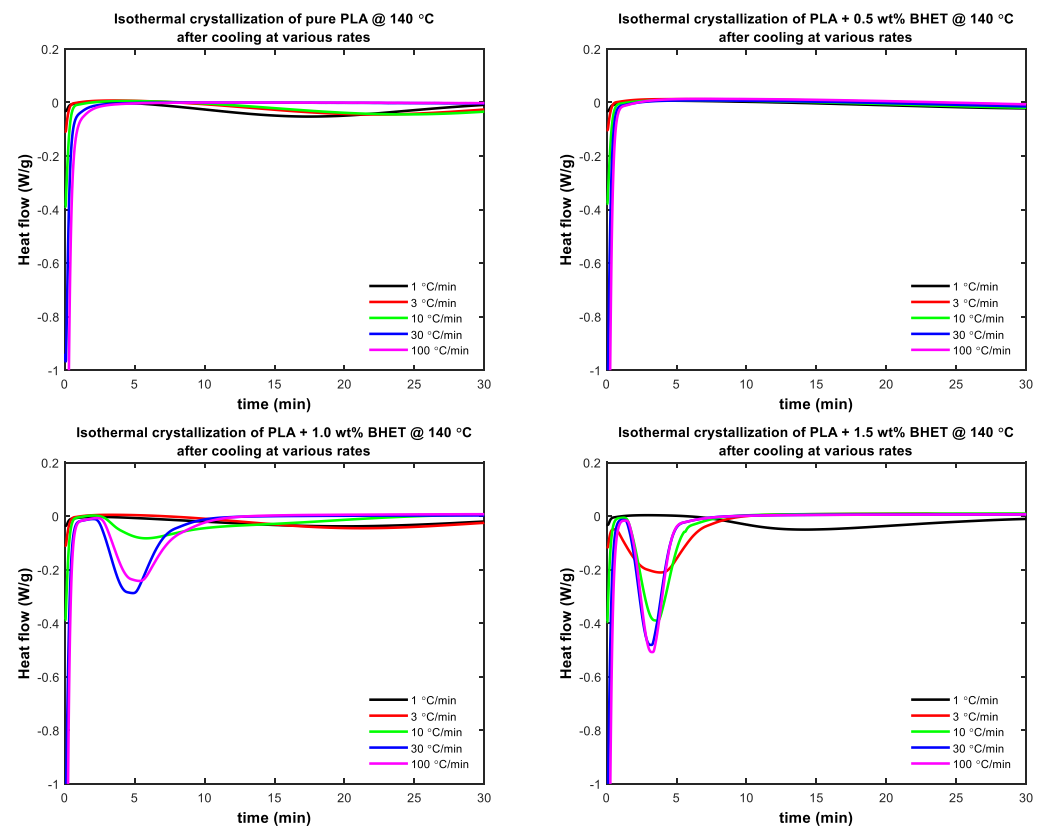

Figure 5.7. DSC thermograms observed during crystallization of PLA (left) and PLA containing $1.5 \mathrm{wt} \%$ BHET (right) under isothermal conditions at $140{ }^{\circ} \mathrm{C}$. Prior to maintaining isothermal conditions, the samples were heated to $200{ }^{\circ} \mathrm{C}$ at a rate of 10 ${ }^{\circ} \mathrm{C} / \mathrm{min}$, after which they were cooled with a specified cooling rate of 1, 3, 10, 30 and $100{ }^{\circ} \mathrm{C} / \min$ to $140{ }^{\circ} \mathrm{C}$.

In contrast, the crystallization of pure PLA and the PLA containing $0.5 \mathrm{wt} \%$ BHET do not show such a cooling rate dependency; instead the crystallization proceeds slowly (pure PLA, Figure 5.7) or not at all in the evaluated isothermal time (0.5 wt\% BHET, Figure 5.7). The absence of notable isothermal crystallization in the presence of $0.5 \mathrm{wt} \%$ BHET is undoubtlely resulting from the inability of BHET to crystallize from the PLA melt under the given conditions combined the plasticising effect. 


\subsection{Conclusions}

In this work we report on the effect of both BHET and BHEF molecules on processing, crystallization, and thermo-mechanical behavior of PLA. We demonstrate that both compounds dissolve in PLA during processing, effectively participating in transesterification reaction with PLA. BHET proves to act as plasticiser for the PLA matrix and proves to be an promising nucleating agent for PLA, though only when it resides in the crystalline form. BHET crystallization favors high cooling rates, as this yields crystallites with a high surface-to-volume ratio. Overall this study demonstrates that the BHET compound, potentially isolated from chemical recycling of thermally cured polymers based on terephthalic acid based bis(2-oxazoline), it a promising additive for use in PLA. It is noteworthly that BHEF, the product that can be isolated after hydrolysis of thermally cured polymers containing a 2,5-furandicarboxylic acid based bis(2-oxazoline) has, despite its renewable origin, no signifnant effect as additive for PLA. Instead, it appears that the use of this renewable monomer is better suited for the development of biobased and biodegradabe polymers rather than for reuse as additive for PLA after chemical recyling.

For additional information, please refer to Appendix D. 


\subsection{References}

(1) Hofvendahl, K.; Hahn-Hägerdal, B. Factors Affecting the Fermentative Lactic Acid Production from Renewable Resources. Enzyme Microb. Technol. 2000, 26 (2-4), 87-107. https://doi.org/10.1016/S0141-0229(99)00155-6.

(2) Cai, H.; Dave, V.; Gross, R. A.; McCarthy, S. P. Effects of Physical Aging, Crystallinity, and Orientation on the Enzymatic Degradation of Poly(Lactic Acid). J. Polym. Sci. Part B Polym. Phys. 1996, 34 (16), 2701-2708. https://doi.org/10.1002/(SICI)10990488(19961130)34:16<2701::AID-POLB2>3.0.CO;2-S.

(3) Garlotta, D. A Literature Review of Poly (Lactic Acid ). 2002, 9 (2).

(4) Abe, H.; Kikkawa, Y.; Inoue, Y.; Doi, Y. Morphological and Kinetic Analyses of Regime Transition of Poly[(S)-Lactide] Crystal Growth. Biomacromolecules 2001, 2 (3), 1007-1014. https://doi.org/10.1021/bm015543v.

(5) Saeidlou, S.; Huneault, M. A.; Li, H.; Park, C. B. Poly(Lactic Acid) Crystallization. Prog. Polym. Sci. 2012, 37 (12), 1657-1677. https://doi.org/10.1016/j.progpolymsci.2012.07.005.

(6) Kopinke, F. D.; Remmler, M.; Mackenzie, K.; Möder, M.; Wachsen, O. Thermal Decomposition of Biodegradable Polyesters - II. Poly(Lactic Acid). Polym. Degrad. Stab. 1996, 53 (3), 329-342. https://doi.org/10.1016/0141-3910(96)00102-4.

(7) Auras, R.; Harte, B.; Selke, S. An Overview of Polylactides as Packaging Materials. Macromolecular Bioscience. September 16, 2004, pp 835-864.

https://doi.org/10.1002/mabi.200400043.

(8) Mai, F.; Tu, W.; Bilotti, E.; Peijs, T. Preparation and Properties of Self-Reinforced Poly(Lactic Acid) Composites Based on Oriented Tapes. Compos. Part A Appl. Sci. Manuf. 2015, 76, 145153. https://doi.org/10.1016/j.compositesa.2015.05.030.

(9) Kang, J. T.; Kim, S. H. Improvement in the Mechanical Properties of Polylactide and Bamboo Fiber Biocomposites by Fiber Surface Modification. Macromol. Res. 2011, 19 (8), 789-796. https://doi.org/10.1007/s13233-011-0807-y.

(10) Park, S. H.; Lee, S. G.; Kim, S. H. Isothermal Crystallization Behavior and Mechanical Properties of Polylactide/Carbon Nanotube Nanocomposites. Compos. PART A 2013, 46, $11-$ 18. https://doi.org/10.1016/j.compositesa.2012.10.011.

(11) Srinivas, V.; van Hooy-Corstjens, C. S. J.; Harings, J. A. W. Correlating Molecular and Crystallization Dynamics to Macroscopic Fusion and Thermodynamic Stability in Fused Deposition Modeling; a Model Study on Polylactides. Polymer (Guildf). 2018, 142, 348-355. https://doi.org/10.1016/j.polymer.2018.03.063.

(12) Marco, C.; Ellis, G.; Gómez, M. A.; Arribas, J. M. Comparative Study of the Nucleation Activity of Third-Generation Sorbitol-Based Nucleating Agents for Isotactic Polypropylene. $J$. Appl. Polym. Sci. 2002, 84 (13), 2440-2450. https://doi.org/10.1002/app.10533.

(13) Balzano, L.; Portale, G.; Peters, G. W. M.; Rastogi, S. Thermoreversible DMDBS Phase Separation in IPP: The Effects of Flow on the Morphology. Macromolecules 2008, 41 (14), 5350-5355. https://doi.org/10.1021/ma7024607. 
(14) Xing, Q.; Li, R.; Dong, X.; Luo, F.; Kuang, X.; Wang, D.; Zhang, L. Enhanced Crystallization Rate of Poly ( $\langle\mathrm{scp}>1</ \mathrm{Scp}\rangle$-Lactide) Mediated by a Hydrazide Compound: Nucleating Mechanism Study. Macromol. Chem. Phys. 2015, 216 (10), 1134-1145.

https://doi.org/10.1002/macp.201500002.

(15) Xu, Y.; Wang, D.; Dong, W.; Chen, M.; Ma, P.; Xu, Y.; Wang, D.; Dong, W.; Chen, M.; Ma, P.; et al. Rapid Crystallization of Poly(Lactic Acid) by Using Tailor-Made Oxalamide Derivatives as Novel Soluble-Type Nucleating Agents. Ind. Eng. Chem. Res. 2014, 53 (32), 12888-12892. https://doi.org/10.1021/ie502211j.

(16) Shen, T.; Xu, Y.; Cai, X.; Ma, P. RSC Advances with Oxalamide Compounds as Nucleators : E Ff Ect of Spacer Length between the Oxalamide Moieties †. 2016, 48365-48374. https://doi.org/10.1039/c6ra04050k.

(17) You, J.; Yu, W.; Zhou, C. Accelerated Crystallization of Poly(Lactic Acid): Synergistic Effect of Poly(Ethylene Glycol), Dibenzylidene Sorbitol, and Long-Chain Branching. Ind. Eng. Chem. Res. 2014, 53 (3), 1097-1107. https://doi.org/10.1021/ie402358h.

(18) Lai, W. C.; Liao, J. P. Nucleation and Crystal Growth Kinetics of Poly(1-Lactic Acid) with Self-Assembled DBS Nanofibrils. Mater. Chem. Phys. 2013, 139 (1), 161-168. https://doi.org/10.1016/j.matchemphys.2013.01.014.

(19) Kawamoto, N.; Sakai, A.; Horikoshi, T.; Urushihara, T.; Tobita, E. Nucleating Agent for Poly(L-Lactic Acid) - An Optimization of Chemical Structure of Hydrazide Compound for Advanced Nucleation Ability. J. Appl. Polym. Sci. 2007, 103 (1), 198-203.

https://doi.org/10.1002/app.25109.

(20) Ma, P.; Deshmukh, Y. S.; Wilsens, C. H. R. M.; Ryan Hansen, M.; Graf, R.; Rastogi, S. SelfAssembling Process of Oxalamide Compounds and Their Nucleation Efficiency in BioDegradable Poly(Hydroxyalkanoate)S. Sci. Rep. 2015, 5 (February), 1-9. https://doi.org/10.1038/srep13280.

(21) Rastogi, S.; Deshmukh, Y.; Piming, M.; Wilsens, C. H. R. M. Nucleating Agents for Polyhydroxyalkanoates. WO 2013/120793 Al, 2013.

(22) Xu, P.; Feng, Y.; Ma, P.; Chen, Y.; Dong, W.; Chen, M. Crystallization Behaviours of Bacterially Synthesized Poly(Hydroxyalkanoate)s in the Presence of Oxalamide Compounds with Different Configurations. Int. J. Biol. Macromol. 2017, 104, 624-630. https://doi.org/10.1016/j.jbiomac.2017.06.001.

(23) Deshmukh, Y. S.; Wilsens, C. H. R. M.; Leone, N.; Portale, G.; Harings, J. A. W.; Rastogi, S. Melt-Miscible Oxalamide Based Nucleating Agents and Their Nucleation Efficiency in Isotactic Polypropylene. Ind. Eng. Chem. Res. 2016, 55 (45), 11756-11766. https://doi.org/10.1021/acs.iecr.6b03120.

(24) Wilsens, C. H. R. M.; Hawke, L. G. D.; Troisi, E. M.; Hermida-Merino, D.; De Kort, G.; Leoné, N.; Saralidze, K.; Peters, G. W. M.; Rastogi, S. Effect of Self-Assembly of Oxalamide Based Organic Compounds on Melt Behavior, Nucleation, and Crystallization of Isotactic Polypropylene. Macromolecules 2018, 51 (13), 4882-4895.

https://doi.org/10.1021/acs.macromol.8b00489.

(25) Blomenhofer, M.; Ganzleben, S.; Hanft, D.; Schmidt, H. W.; Kristiansen, M.; Smith, P.; Stoll, K.; Mäder, D.; Hoffmann, K. "Designer" Nucleating Agents for Polypropylene. 
Macromolecules 2005, 38 (9), 3688-3695. https://doi.org/10.1021/ma0473317.

(26) Varga, J.; Menyhárd, A. Effect of Solubility and Nucleating Duality of N,N'-Dicyclohexyl-2,6Naphthalenedicarboxamide on the Supermolecular Structure of Isotactic Polypropylene. Macromolecules 2007, 40 (7), 2422-2431. https://doi.org/10.1021/ma062815j.

(27) Shi, Y.; Xin, Z. Study on Variable Nucleation Efficiency of $N, N^{\prime}$-Dicyclohexyl-2,6Naphthalenedicarboxamide on Isotactic Polypropylene. J. Thermoplast. Compos. Mater. 2016, 29 (12), 1667-1679. https://doi.org/10.1177/0892705715583178.

(28) Yang, J.; Liang, R.; Chen, Y.; Zhang, C.; Zhang, R.; Wang, X.; Kong, R.; Chen, Q. Using a Self-Assemblable Nucleating Agent to Tailor Crystallization Behavior, Crystal Morphology, Polymorphic Crystalline Structure, and Biodegradability of Poly(1,4-Butylene Adipate). Ind Eng. Chem. Res. 2017, 56 (28), 7910-7919. https://doi.org/10.1021/acs.iecr.7b01783.

(29) Zhang, J.; Tashiro, K.; Tsuji, H.; Domb, A. J. Disorder-to-Order Phase Transition and Multiple Melting Behavior of Poly(L-Lactide) Investigated by Simultaneous Measurements of WAXD and DSC. Macromolecules 2008, 41 (4), 1352-1357. https://doi.org/10.1021/ma0706071.

(30) Hoogsteen, W.; Postema, A. R.; Pennings, A. J.; Brinke, G. Ten; Zugenmaier, P. Crystal Structure, Conformation, and Morphology of Solution-Spun Poly(L-Lactide) Fibers. Macromolecules 1990, 23 (2), 634-642. https://doi.org/10.1021/ma00204a041.

(31) Fillon, B.; Lotz, B.; Thierry, A.; Wittmann, J. C. Self-Nucleation and Enhanced Nucleation of Polymers. Definition of a Convenient Calorimetric "Efficiency Scale" and Evaluation of Nucleating Additives in Isotactic Polypropylene ( $\alpha$ Phase). J. Polym. Sci. Part B Polym. Phys. 1993, 31 (10), 1395-1405. https://doi.org/10.1002/polb.1993.090311014.

(32) Feng, Y.; Ma, P.; Xu, P.; Wang, R.; Dong, W.; Chen, M.; Joziasse, C. The Crystallization Behavior of Poly(Lactic Acid) with Different Types of Nucleating Agents. Int. J. Biol. Macromol. 2018, 106, 955-962. https://doi.org/10.1016/j.ijbiomac.2017.08.095. 



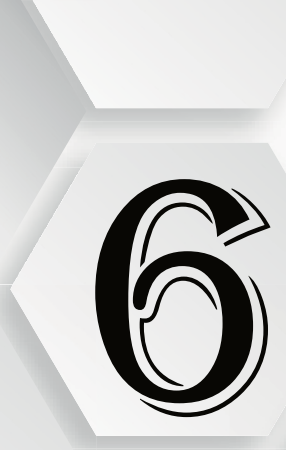

\section{ON THE NUCLEATION OF POLYLACTIDE BY MELT-SOLUBLE OX ALAMIDE BASED ORGANIC COMPOUNDS}

Chapter partly based on:

Roy, M; Zhelezniakov, M; De Kort, G; Hawke, L; Leoné, N; Rastogi, S; Wilsens, C.H.R.M: On the nucleation of polylactide by melt-soluble oxalamide based organic compounds,'Polymer, 2020, 122680.

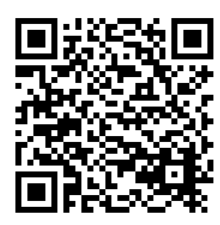

Special thanks to Maxhim Zhelezniakov for his hard work and perseverance and all the co-authors for their contribution in the successful completion of this work. 


\title{
In this chapter...
}

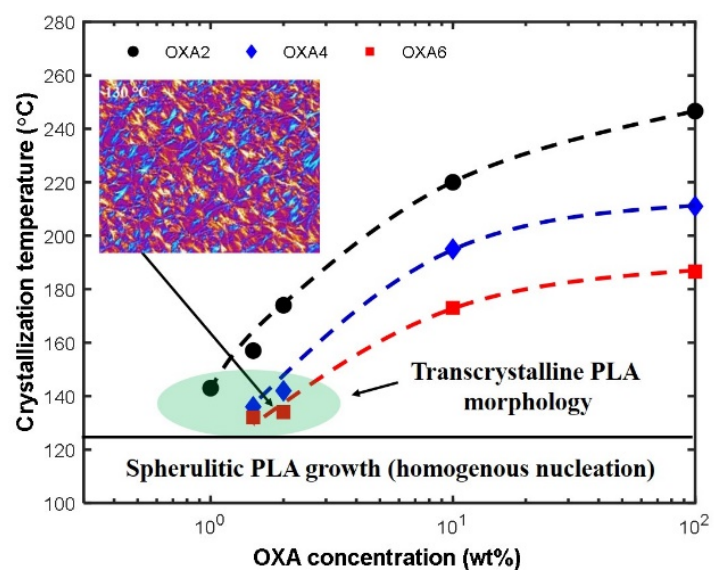

"Self-assembly of bis(oxalamide) based organic compounds enhancing the nucleating efficiency of PLA"

\begin{abstract}
The aim of this chapter is to evaluate various nucleation mechanisms and identify the critical parameters responsible for the enhancement of the PLA nucleation process in the presence of oxalamide based organic compounds (OBOC)s. The chapter addresses the mechanism involved in the cooling rate dependence on the nucleation efficiency of the OBOC: The findings override the possibility of both epitaxy and soft epitaxy as a plausible nucleation mechanism, making both the surface roughness of OBOC crystals and the local stresses imposed on the PLA melt proceeding from a highly supersaturated state as explanation to the cause of the favored crystallization of PLA at the high cooling rates in the presence of the chosen oxalamides.
\end{abstract}




\subsection{Introduction}

Polylactide (PLA) has attracted considerable attention in the recent years, resulting from its renewable origin ${ }^{1-4}$, biocompatibility ${ }^{5,6}$ and biodegradability ${ }^{7}$ Nevertheless, though PLA is considered a promising renewable polymer, it suffers from brittleness, low impact resistance, and poor long-term behavior due to its pronounced creep $^{8,9}$. The latter is mostly the result of the low crystallinity of PLA after processing, resulting from its slow nucleation and crystallization kinetics ${ }^{10}$. Though the supercooling provides the driving force for the homogenous nucleation and crystallization process of PLA, the nucleation process can be enhanced by the addition of a nucleation agent ${ }^{11-13}$ (NA), potentially addressing the aforementioned limits in mechanical performance.

Numerous additives, for example, calcium carbonate ${ }^{14}$, cellulose ${ }^{15}$, clay ${ }^{16}$, talc $^{17}$, functionalized lignin ${ }^{18}$, hydrazides ${ }^{19,20}$, amino-acids ${ }^{21}$, and oxalamides ${ }^{22}$ have been reported to enhance the nucleation rate of the PLA melt. When using inorganic compounds, irrespective of the nucleation mode, the observed nucleating efficiency is generally limited by the (in)homogeneity of the dispersion of the filler in the polymer matrix. In this respect, the use of melt-soluble organic nucleating agents is preferred. These organic molecules are designed to dissolve in the polymer melt and crystallize on cooling - prior to crystallization of the polymer melt. It is to be realized that on decreasing the temperature of the polymer/NA solution, the solubility of the NA decreases until a supersaturated state is achieved that promotes nucleation and crystallization of the NA; a process that is dependent on both the temperature and the NA concentration in the polymer melt. Crystallization of the NA from such a supersaturated state effectively yields a homogenous dispersion of NA crystals throughout the polymer matrix. In turn, the overall nucleating efficiency of the polymer matrix is determined by the geometry and the surface-to-volume ratio of the NA crystals, which in turn is dependent on the thermal history of the sample, and hence the supersaturation of the system prior to the NA crystallization.

An example of melt-soluble nucleating agents is oxalamide based organic compounds (OBOC). Generally, two oxalamide moieties are connected through a bridging spacer, which is varied to control the OBOC melting temperature. Secondly, the end-groups of the OBOC are selected such that they promote miscibility with the polymer matrix of choice. When designed correctly and used in sufficient concentration, the side groups stimulate dissolution in the polymer matrix at elevated temperatures, while the hydrogen bonding between the oxalamide moieties provides the driving force for crystallization from the polymer melt upon cooling ${ }^{23,24}$. Indeed, this approach proved successful in designing melt-soluble nucleating agents for

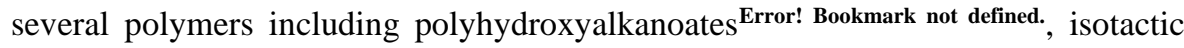


polypropylene ${ }^{24,25}$, polylactide ${ }^{22,26}$, and poly(butylene-adipate $)^{26,27}$. Though this generic approach in designing nucleating agents for thermoplastic polymers is promising, the mode of nucleation is not clear; Given that multiple OBOCs can effectively enhance the nucleation efficiency of different polymers, we consider nucleation by epitaxial matching as general OBOC nucleation mechanism unlikely. Therefore, the purpose of this work is to evaluate various nucleation mechanisms and identify the critical parameters responsible for the enhancement of the PLA nucleation process in the presence of OBOCs.

\subsection{Experimental section}

\subsubsection{Material Preparation and Blending Process}

Five different OBOCs have been synthesized according to the procedure

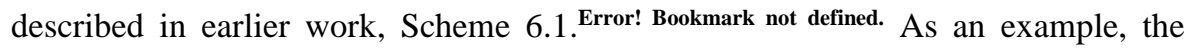
synthesis of diethyl 5,6,11,12-tetraoxo-4,7,10,13-tetraazahexadecane-1,16-dioate, abbreviated as OXA2, is briefly recalled; 1,2-diaminoethane was dropwise added to a 10-fold excess of diethyl oxalate in THF and the mixture was allowed to stir for 24 hours at room temperature. The intermediate product, diethyl 2,2'-(ethane-1,2diylbis(azanediyl))bis(2-oxoacetate), precipitated during the reaction and was obtained as a white powder after filtration and washing three times with diethyl ether. Next, diethyl 2,2'-(ethane-1,2-diylbis(azanediyl))bis(2-oxoacetate) was dissolved in chloroform together and a double molar excess of $\beta$-alanine ethyl ester hydrochloride and triethyl amine. The product, OXA2, precipitated over time during refluxing for 48 hours and was obtained as a white powder after filtration and washing three times with chloroform.

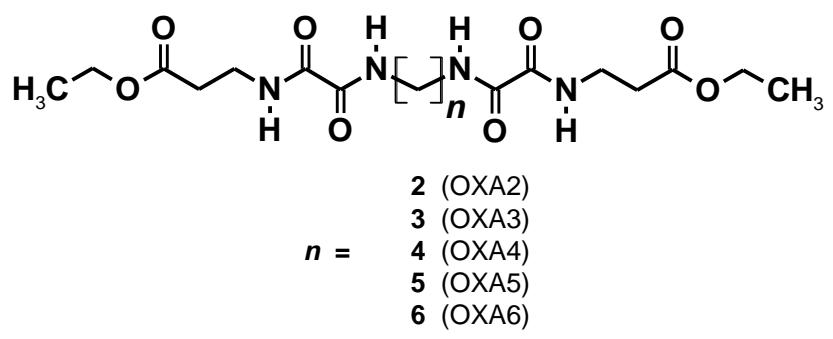

Scheme 6.1. Chemical structures of the five OBOCs used in this study. The OBOCs are abbreviated based on the number of methylene groups in-between the oxalamide units; For example, the OBOC based on 1,2-ethanediamine, having two methylene groups between the oxalamide groups, is named OXA2. 
The thermal behavior of the OBOCs synthesized in this study, having 2, 3, 4, 5, and 6 methylene spacers between the oxalamide groups, has been assessed using differential scanning calorimetry at heating and cooling rates of $10{ }^{\circ} \mathrm{C} / \mathrm{min}$ (Figure 6.1 , top). An overview of the peak melting and crystallization temperatures are displayed in Figure 6.1, bottom. In general, we observe that an increase in spacer length results in a decrease in melting temperature, on top of the odd-even effect: OBOC having an odd number of methylene segments between the oxalamide groups display a significantly suppressed melting and crystallization temperature. Furthermore, for most OBOCs we observe various crystal-to-crystal transitions prior to final melting. Lastly, we observe that all OBOCs start to degrade either at temperatures above $200{ }^{\circ} \mathrm{C}$ or above their melting temperature ${ }^{23}$.
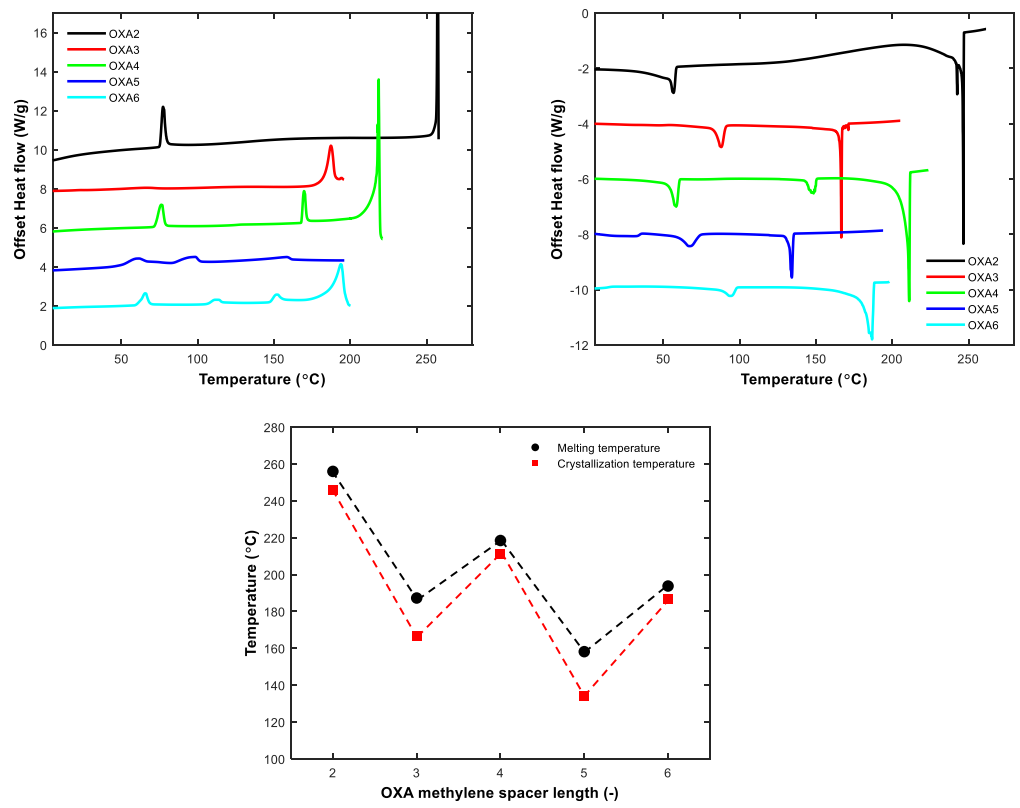

Figure 6.1. DSC heating traces (top, left) and cooling traces (top, right) of the pure OBOCs synthesized in this study, obtained at heating and cooling rates of $10^{\circ} \mathrm{C} / \mathrm{min}$. Additionally, the peak melting and crystallization temperatures as observed from the top images, depicting a clear odd-even effect, are provided as a function of OXA methylene spacer length in the bottom image.

The synthesized OBOCs are dried overnight at $60^{\circ} \mathrm{C}$ in vacuo together with the PLA (Corbion L130 grade, <1\% d-enantiomer). Next, master batches of $10 \mathrm{wt} \%$ OBOC in PLA is prepared through extrusion for three minutes in a DSM Xplore twinscrew extruder ( $5 \mathrm{ml}$ barrel size) at $180{ }^{\circ} \mathrm{C}$ and at 100 RPM. Next, the master-batches are diluted with PLA to obtain samples of $0.5,1.0,1.5$, and $2.0 \mathrm{wt} \%$ OBOC. After 
extrusion, the samples are cooled to room temperature after which they were subjected to further analysis. A pure PLA reference is processed following the same protocol.

\subsubsection{Analysis methods}

${ }^{1} \mathrm{H}-\mathrm{NMR}$ spectra were recorded with a Bruker Ultrashield 300 spectrometer (300 MHz magnetic field). NMR-samples were prepared by dissolving ca. $10 \mathrm{mg}$ of sample in $0.5 \mathrm{ml}$ deuterated dimethyl sulfoxide (DMSO). Spectra were referenced against tetramethyl silane (TMS).

Weight average molecular weight $\left(M_{\mathrm{w}}\right)$ and polydispersity (PDI) of PLA blends after processing were determined from gel permeation chromatography (GPC) on a PSS SECcurity GPC system using Agilent 1260 Infinity instrument technology. The GPC system is equipped with two PFG combination medium micro-columns with $7 \mu \mathrm{m}$ particle size $(4.6 \times 250 \mathrm{~mm}$, separation range $100-1.000 .000 \mathrm{Da})$, a PFG combination medium pre-column with $7 \mu \mathrm{m}$ particle size $(4.6 \times 30 \mathrm{~mm})$, and a Refractive Index detector (RI). Distilled 1,1,1,3,3,3-hexafluoroisopropanol (HFIP) containing $0.019 \%$ sodium trifluoroacetate was used as mobile phase at $40{ }^{\circ} \mathrm{C}$, with a $0.3 \mathrm{~mL} / \mathrm{min}$ flow rate. The GPC apparatus was calibrated with poly(methyl methacrylate) standards obtained from PSS. GPC samples were prepared by dissolving $5 \mathrm{mg}$ of PLA in $1.5 \mathrm{ml}$ HFIP overnight under constant shaking, the samples were filtered over a $0.2 \mu \mathrm{m}$ PTFE syringe filter prior to injection.

The thermal behavior and transitions of the samples were identified via differential scanning calorimetry using a TA Instruments Q2000 DSC. All samples were measured at heating rate of $10^{\circ} \mathrm{C}$ to a maximum temperature of $190{ }^{\circ} \mathrm{C}$, kept isothermal for 3 minutes, and cooled back to room temperature at a rate of 1,3, 10, 30 , or $100{ }^{\circ} \mathrm{C} / \mathrm{min}$ unless mentioned otherwise. The glass transition temperature $\left(T_{\mathrm{g}}\right)$ and peak melting temperature $\left(T_{\mathrm{m}}\right)$ were determined from heating runs. The crystallization exotherm observed during the first cooling run was used to define peak crystallization temperature $\left(T_{\mathrm{c}}\right)$ and onset of crystallization temperature $\left(T_{\mathrm{ons}}\right)$. The peak crystallization temperature is considered the peak value of the crystallization exotherm, whereas the intersection of the tangents of the baseline and the crystallization curve is considered the onset temperature for crystallization. For isothermal crystallization, all samples are heated from room temperature to $190{ }^{\circ} \mathrm{C}$ at a rate of $10{ }^{\circ} \mathrm{C} / \mathrm{min}$ and kept isothermal for 3 minutes to erase the thermal history. Next, these samples are cooled at a rate of $50{ }^{\circ} \mathrm{C} / \mathrm{min}$ to the desired isothermal holding temperature (between $145-170{ }^{\circ} \mathrm{C}$ ) to allow nucleation and crystallization of the dissolved OXA molecules. After a five-minute isotherm at the holding temperature, the samples were cooled at a rate of $50{ }^{\circ} \mathrm{C} / \mathrm{min}$ to $140{ }^{\circ} \mathrm{C}$ after which the isothermal crystallization of the PLA was monitored for one hour. Reference samples were 
measured without subjection to an isothermal holding temperature, i.e. they were directly cooled to $140{ }^{\circ} \mathrm{C}$ at a rate of $50{ }^{\circ} \mathrm{C} / \mathrm{min}$ after which the isothermal crystallization of the PLA was monitored. The obtained crystallization isotherms were subjected to an Avrami analysis ${ }^{28}$, The enthalpy of crystallization exotherm as a function of the isothermal time $\left(\Delta \mathrm{H}_{(\mathrm{t})}\right.$ in $\left.\mathrm{J} / \mathrm{g}\right)$ was divided over the total of the crystallization enthalpy ( $\Delta H_{\text {total }}$ in $\mathrm{J} / \mathrm{g}$ ) to yield the crystalline mass fraction $W_{\mathrm{c}}$. Note, the crystallization time was taken as $t-t_{0}$ where $t_{0}$ denotes that starting time of the crystallization exotherm. Next, the crystalline mass fraction was converted to the crystalline volume fraction $\left(V_{\mathrm{c}}\right)$, using equation (6.1),

$$
V_{c}=\frac{W_{c}}{W_{c}+\left(\frac{\rho_{c}}{\rho_{a}}\right)\left(1-W_{c}\right)}
$$

where the $\rho_{\mathrm{c}}$ and $\rho_{\mathrm{a}}$ are the crystalline and amorphous densities of PLA, being 1.267 $\mathrm{g} / \mathrm{cm}^{3}$ and $1.257 \mathrm{~g} / \mathrm{cm}^{3}$, respectively ${ }^{29}$. The Avrami parameters $\mathrm{n}$ (dimensionless) and $\mathrm{k}$ (in $\mathrm{min}^{-\mathrm{n}}$ ) can be extracted from the Avrami equation (6.2) after applying logarithmic properties to both sides, equation $(6.3)^{30}$

$$
\begin{gathered}
1-V_{c}=\exp \left(-k t^{n}\right) \\
\log \left[-\ln \left[1-V_{c}(t)\right]\right]=\log (k)+n \log (t)
\end{gathered}
$$

Classically, the Avrami parameter $\mathrm{n}$ is the summation of two terms, $\mathrm{n}_{\mathrm{d}}$ and $\mathrm{n}_{\mathrm{n}}$, where $\mathrm{n}_{\mathrm{d}}$ reflects the dimensionality of the growting crystals $(1,2$, or 3$)$, and $\mathrm{n}_{\mathrm{n}}$ reflects the nucleation dependency which should vary between 0 (instantaneous nucleation) and 1 (sporadic nucleation) ${ }^{30}$.

The morphological development during crystallization of the samples was monitored using polarized optical microscopy on an Olympus BX53 microscope mounted with an Olympus DP26 camera and a Linkam hotstage (between crosspolarizers and using a $530 \mathrm{~nm} \lambda$-wave plate). The samples were heated to the desired temperature (generally $200{ }^{\circ} \mathrm{C}$ ) at a rate of $30{ }^{\circ} \mathrm{C} / \mathrm{min}$. After leaving the samples for 3 min under isothermal conditions, the samples were cooled at a specified cooling rate (generally being 3,10 , or $30^{\circ} \mathrm{C} / \mathrm{min}$ ) to the desired temperature where isothermal crystallization was monitored for 30 minutes. Optical micrographs depicting the morphological changes were recorded both during cooling and under isothermal conditions.

The evolution of the storage modulus of the PLA blends was followed over time at $200{ }^{\circ} \mathrm{C}$ in an Anton Paar 702 twin drive rheometer. A parallel plate geometry, having a diameter of $15 \mathrm{~mm}$ and gap of $750 \mu \mathrm{m}$, was used to study the time dependence of the storage modulus over temperature range from $200{ }^{\circ} \mathrm{C}$ to $80{ }^{\circ} \mathrm{C}$. 
The strain (1\%) and angular frequency (10 rad/s) were chosen such that the experiments were performed in the linear viscoelastic regime.

\subsection{Results and discussion}

\subsubsection{OBOC as melt-miscible nucleating agent for PLA}

The OBOCs synthesized in this study (Scheme 6.1) are designed such that they dissolve in the PLA melt at elevated temperatures and crystallize upon cooling. To ensure the interaction required for dissolution in PLA at elevated temperatures, the OBOCs contain two $\beta$-alanine ethyl ester end-groups, which are expected to exhibit a high affinity towards the PLA chains due to their structural similarity. Secondly, the introduction of the hydrogen bonding oxalamide motifs provides the driving force to crystallize during cooling. These two features, i.e. dissolution and crystallization of the OBOCs are clearly observed in optical microscopy: An example of the capability of the synthesized OBOCs to enhance the nucleation process for PLA is provided in Figure 6.2, where the crystallization process of PLA in the presence of $2.0 \mathrm{wt} \%$ OXA4 is visualized during cooling.

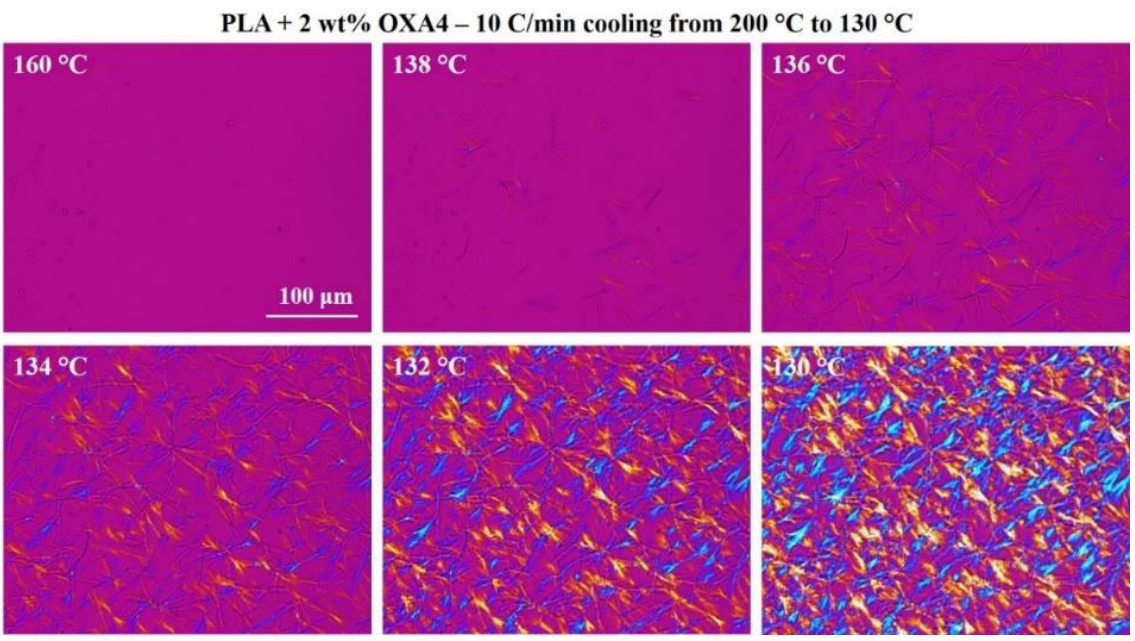

Figure 6.2. Polarization optical micrographs depicting the morphological changes when cooling the sample containing PLA and $2.0 \mathrm{wt} \%$ OXA4 from $200{ }^{\circ} \mathrm{C}$ to $130^{\circ} \mathrm{C}$ at a rate of $10^{\circ} \mathrm{C} / \mathrm{min}$.

As observed from figure 6.2, the OXA4 dissolves in the PLA melt, as a homogeneous mixture is obtained above the melting temperature of the PLA crystals. The crystallization of OXA4 molecules into star-shaped crystals is detected around $138-136{ }^{\circ} \mathrm{C}$ during cooling at a rate of $10^{\circ} \mathrm{C} / \mathrm{min}$, whereas the growth of the PLA crystals on the surface of the OXA 4 crystals is immediately observed upon further 
cooling. Interestingly, we observe the formation of a transcrystalline PLA morphology growing from the surface of the OXA4 crystals instead of spherulitic growth, indicated by the matching of the colors of the OXA4 crystals and the PLA crystals growing on their surface.

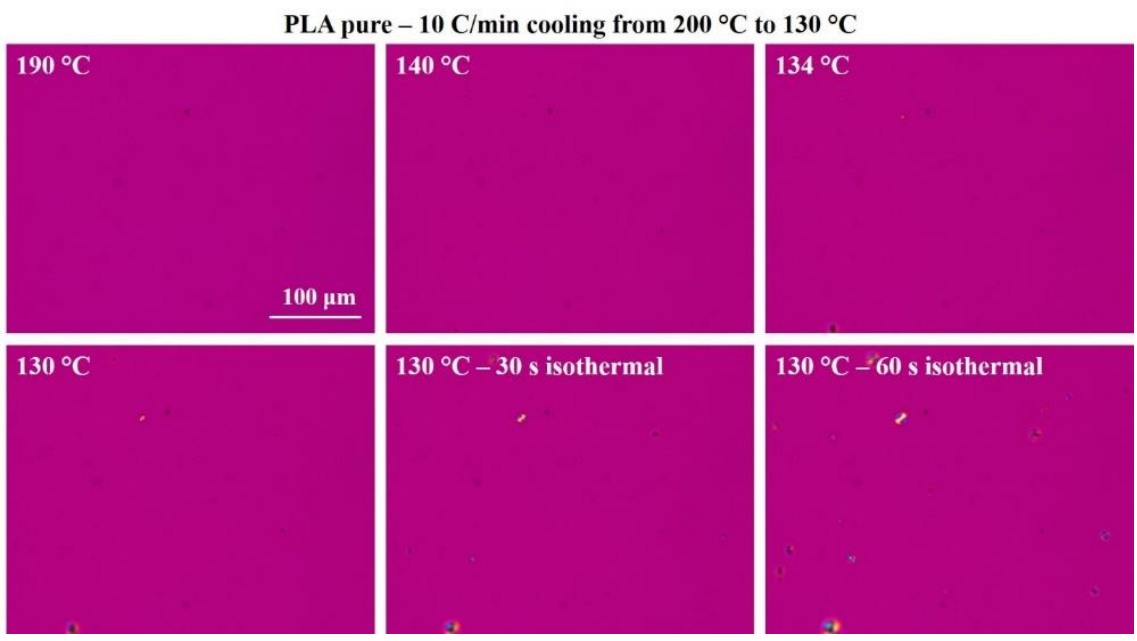

Figure 6.3. Polarization optical micrographs depicting the morphological changes when cooling the pure PLA sample from $200{ }^{\circ} \mathrm{C}$ to $130{ }^{\circ} \mathrm{C}$ at a rate of $10{ }^{\circ} \mathrm{C} / \mathrm{min}$. Upon reaching $130{ }^{\circ} \mathrm{C}$, the sample was kept isothermal for 1 minute while the changes in optical morphology were monitored.

Overall, this example clearly demonstrates that OXA4 crystals can effectively enhance the nucleation process of PLA, as the pure PLA sample does not display any notable crystallization when subjected to the same experimental protocol (Figure 6.3). The nucleating efficiency of the remaining OXA compounds as well as the main factors that govern it are detailed in the section below.

\subsubsection{Effect of the OBOC crystallization temperature on transcrystalline PLA morphology}

Strikingly different results are obtained when the same experimental protocol is followed for PLA containing $2.0 \mathrm{wt} \%$ OXA2 (Figure 6.4). In contrast to OXA4, Figure 6.4 reveals that the OXA2 crystal growth proceeds already at $174{ }^{\circ} \mathrm{C}$ during cooling at a rate of $10{ }^{\circ} \mathrm{C} / \mathrm{min}$ and that the OXA2 crystals grow into relatively large needle shaped crystals instead of star-shaped crystals. More importantly and in stark contrast to the $2.0 \mathrm{wt} \%$ OXA4 system, further cooling of the PLA sample containing $2.0 \mathrm{wt} \%$ OXA2 only results in the formation of a low number of PLA spherulites at $130{ }^{\circ} \mathrm{C}$. The absence of the previously observed transcrystalline PLA 
morphology suggests that the OXA2 is a poor nucleating agent under the chosen experimental conditions.

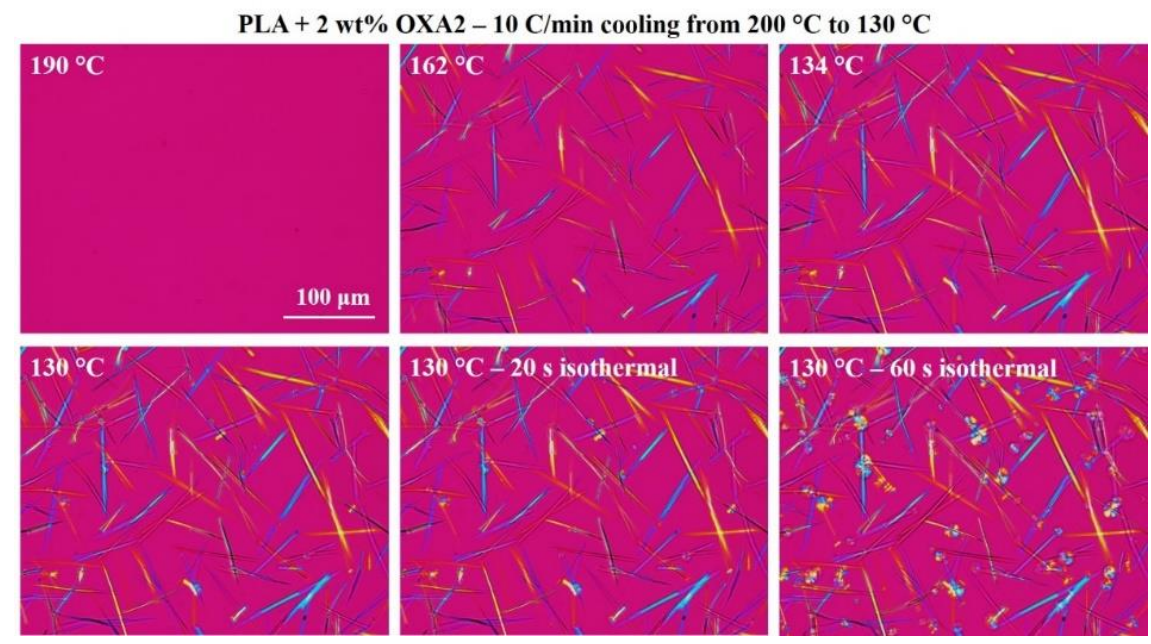

Figure 6.4. Polarization optical micrographs depicting the morphological changes when cooling the sample containing PLA and $2.0 \mathrm{wt} \% \mathrm{OXA} 2$ from $200{ }^{\circ} \mathrm{C}$ to $130{ }^{\circ} \mathrm{C}$ at a rate of $10^{\circ} \mathrm{C} / \mathrm{min}$. Upon reaching $130^{\circ} \mathrm{C}$, the sample was kept isothermal for 1 minute while the changes in optical morphology were monitored.

In contrast, when the OXA2 concentration is decreased to $1.0 \mathrm{wt} \%$, OXA2 crystallization is observed at considerably lower temperatures of $143{ }^{\circ} \mathrm{C}$ (Figure 6.5), which is immediately followed by transcrystallization of PLA from the OXA 2 crystal surface. These findings indicate that the nucleating efficiency of PLA in the presence of OXA2 increases when the crystallization temperature of the OXA2 molecules from the PLA melt decreases. 


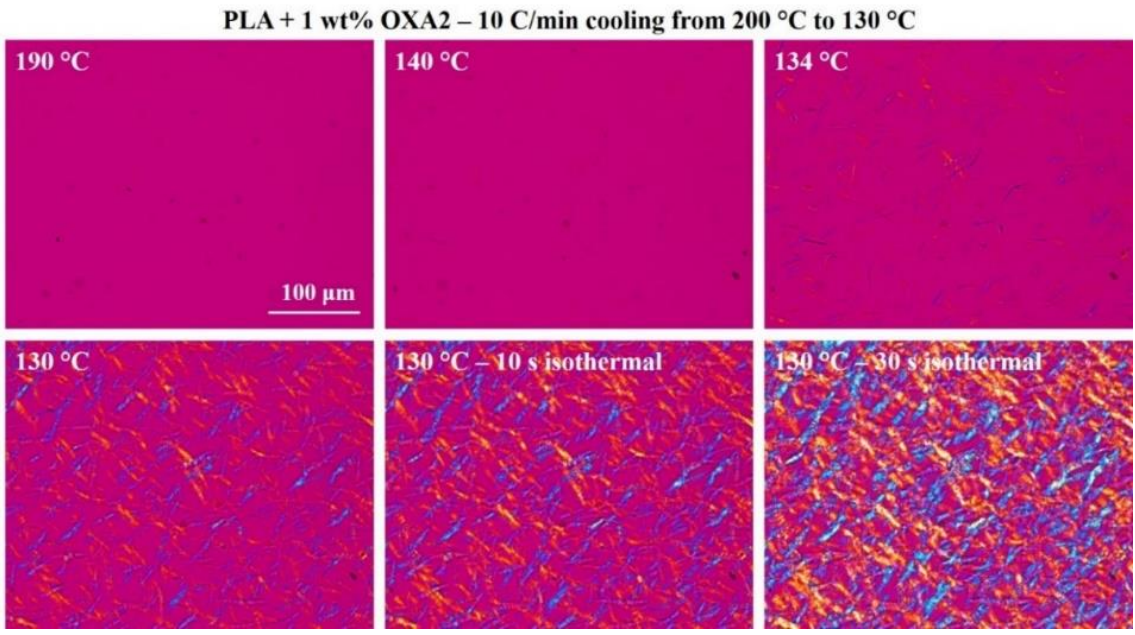

Figure 6.5. Polarization optical micrographs depicting the morphological changes when cooling the sample containing PLA and $1.0 \mathrm{wt} \% \mathrm{OXA} 2$ from $200{ }^{\circ} \mathrm{C}$ to $130{ }^{\circ} \mathrm{C}$ at a rate of $10^{\circ} \mathrm{C} / \mathrm{min}$. Upon reaching $130^{\circ} \mathrm{C}$, the sample was kept isothermal for 1 minute while the changes in optical morphology were monitored.

Similarly, when subjecting PLA containing $2.0 \mathrm{wt} \%$ OXA6 to the same experimental protocol, OXA6 crystallization is observed at $132{ }^{\circ} \mathrm{C}$, immediately followed by PLA transcrystallization from the OXA6 crystal surface (Figure 6.6). Overall, these findings support the notion that the PLA nucleating efficiency by the OBOCs is strongly dependent on the crystallization temperature of the OBOC molecules from the PLA melt, hence on the supersaturation of the OBOC solution upon crystallization.

In order to identify the interplay between the employed OBOC concentration and the melting temperature of the pure OBOC compounds, we have compiled a phase diagram depicting the dependency of the OBOC crystallization temperature from the PLA melt as a function of OBOC concentration (Figure 6.7). Interestingly, no crystallization of the OXA3 and OXA5 was observed from the PLA melt upon cooling, likely originating from the low melting temperatures of the pure compounds $\left(187^{\circ} \mathrm{C}\right.$ and $155^{\circ} \mathrm{C}$, respectively, Figure 6.1). For this reason, Figure 6.7 only reflects systems containing OXA2, OXA4, and OXA6. In general, we observe that the OBOC crystallization temperature from the PLA melt decreases with decreasing concentration. Furthermore, as expected for miscible systems displaying a melting and crystallization temperature suppression, the phase diagram shows that the crystallization temperature follows the order OXA2 > OXA4 > OXA6 for a given OBOC concentration. Note that no OBOC crystallization was observed from the PLA in melt samples containing $\leq 0.5 \mathrm{wt} \%$ OXA2 and $\leq 1.0 \mathrm{wt} \%$ OXA4 or OXA6. We 
expect that the OBOC crystallization from the PLA melt at such low concentrations proceeds only after crystallization of the PLA has occurred, and hence it is not observed under the chosen experimental conditions. The circled area denotes the combinations of OBOC concentrations and crystallization temperatures where a transcrystalline PLA morphology develops on the OBOC crystals surface during cooling. Overall, Figure 6.7 shows that the enhancement in nucleating efficiency of PLA, indicated by the development of the transcrystalline PLA morphology, proceeds when the OBOC crystallizes around or below $145^{\circ} \mathrm{C}$. Systems for which OBOC crystallization sets off at higher temperatures do not exhibit such a significant enhancement in PLA nucleating efficiency, and a spherulitic crystallization morphology, similar to those displayed in Figure 6.4, is observed instead.

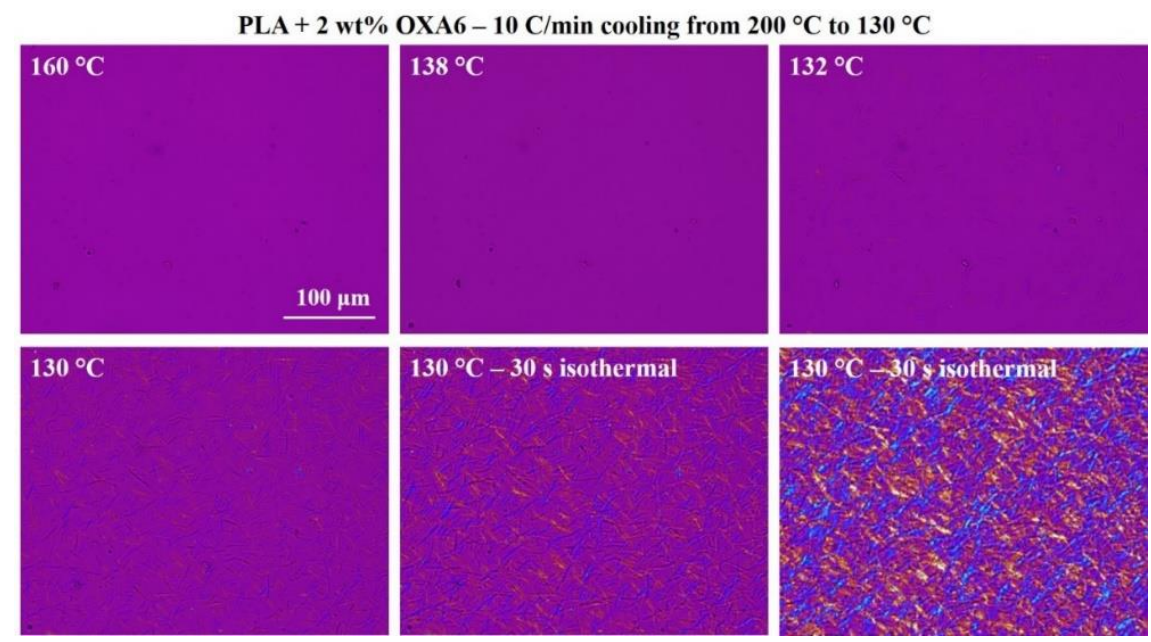

Figure 6.6. Polarization optical micrographs depicting the morphological changes when cooling the sample containing PLA and $2.0 \mathrm{wt} \%$ OXA6 from $200{ }^{\circ} \mathrm{C}$ to $130{ }^{\circ} \mathrm{C}$ at a rate of $10^{\circ} \mathrm{C} / \mathrm{min}$. Upon reaching $130^{\circ} \mathrm{C}$, the sample was kept isothermal for 1 minute while the changes in optical morphology were monitored. 


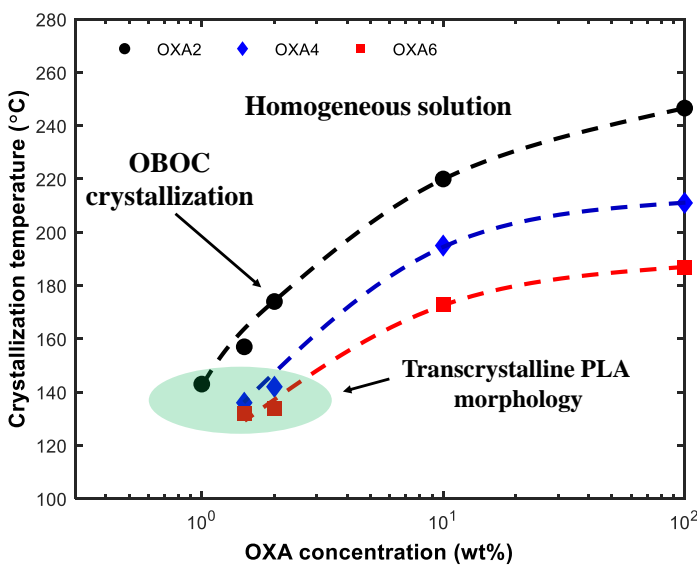

Figure 6.7. Phase diagram depicting the crystallization temperature of OXA2, OXA4, and OXA6 from the PLA melt at various concentrations, as determined from polarized optical microscopy. The crystallization temperature reflects the visual observation of the $\mathrm{OBOC}$ crystals during cooling from the melt at a rate $10^{\circ} \mathrm{C} / \mathrm{min}$. The region highlighted in green reflects the concentration range where a transcrystalline PLA morphology is observed on the OBOC crystal surface upon continued cooling. Note, the dotted lines are added to guide the eye of the reader.

The aforementioned findings from polarized optical microscopy correspond well with findings from DSC. As can be observed from Figure 6.8, left, no notable increase in peak crystallization temperature $\left(T_{\mathrm{c}}\right)$ of PLA is observed for the PLA containing $0.5 \mathrm{wt} \%$ OXA2 $\left(T_{\mathrm{c}}\right.$ of $100{ }^{\circ} \mathrm{C}$, compared to $99{ }^{\circ} \mathrm{C}$ for the pure PLA sample). As observed earlier, this directly relates to the inability of the OXA2 to crystallize from the PLA melt under these conditions, resulting in the homogeneous nucleation of PLA. As expected from Figure 6.5, a significant increase in $T_{\mathrm{c}}$ to $115^{\circ} \mathrm{C}$ is observed in the PLA containing $1.0 \mathrm{wt} \%$ OXA2. Similarly, when increasing the OXA2 concentration further, the $T_{\mathrm{c}}$ of PLA is observed at 102 (1.5 wt\% OXA2) and $101{ }^{\circ} \mathrm{C}(2.0 \mathrm{wt} \%$ OXA2), undoubtedly resulting from the fact the OXA2 crystal were generated at too high temperatures. In addition, as is evident from Figure 6.4, the generation of the OXA crystals in systems containing 1.5 and $2.0 \mathrm{wt} \%$ OXA2 results in a relatively poor dispersion of large OXA2 particles with a decreased surface-tovolume ratio (as is evident when comparing Figures 6.4 and 6.5), inevitably decreasing the number of potential heterogeneous nucleation sites further. 

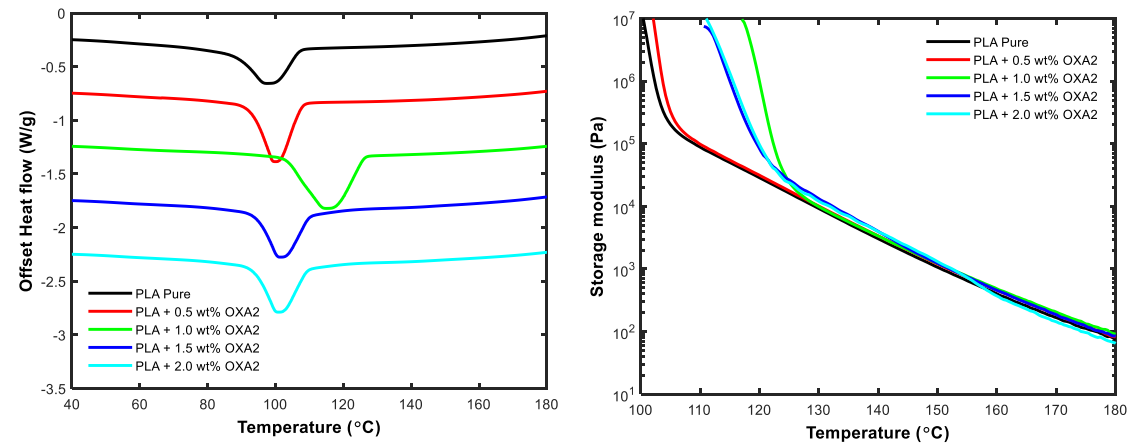

Figure 6.8. Left, first DSC cooling traces taken at a cooling rate of $10^{\circ} \mathrm{C} / \mathrm{min}$ for systems having varying concentration of OXA2, depicting the characteristic crystallization exotherms. Right, the evaluation of the storage modulus observed during cooling at a rate of $5{ }^{\circ} \mathrm{C} / \mathrm{min}$ for systems having varying OXA2 concentration.

The trends are also observed in rheology (Figure 6.8, right). Note that, when PLA samples containing OXA2 are cooled from the melt, the sudden deviation from Arrhenius type of behavior (pure PLA, Figure 6.8 right) can be identified as the onset of PLA crystallization ( $\left.T_{\mathrm{ons}}\right)$. The sample containing $1.0 \mathrm{wt} \%$ OXA2 shows the highest $T_{\text {ons }}$ of $125^{\circ} \mathrm{C}$, while the $T_{\text {ons }}$ decreases in the samples containing 1.5 and 2.0 wt $\%$ OXA2 to 123 and $122{ }^{\circ} \mathrm{C}$, respectively. More importantly, one can observe that the slope of the storage modulus during cooling to temperatures below $T_{\text {ons }}$ is lower for samples containing 1.5 and $2.0 \mathrm{wt} \% \mathrm{OXA} 2$, suggesting a slower increase in crystalline fraction, hence these systems contain a lower number of growing PLA crystals. As mentioned earlier, this is likely the result from the poorer OXA2 distribution, its lowered surface-to-volume ratio and the fact that the OXA2 crystals were grown at too high temperatures. For the sample containing $2.0 \mathrm{wt} \%$ OXA2, the crystallization of the OXA2 itself can be detected by the slight stepwise increase of the storage modulus around $160{ }^{\circ} \mathrm{C}$.

\subsubsection{PLA crystallization during cooling in the presence of $O B O C$ in varying concentration}

The DSC and rheology data for the PLA samples with varying concentrations of the other OBOCs evaluated in this study are provided below in figure 6.9 and figure 6.10. It is evident from figure 6.9, that low melting temperature OBOCs (OXA3 and OXA5) does not hold the capacity to enhance the nucleation of PLA crystallites, whereas, the high melting OBOCs showed significant efficiency towards enhancing the nucleation of PLA crystallites. However, both the Overall, these findings confirm that the capability of the OBOCs to enhance the nucleation efficiency of PLA is highest when the OBOC crystallizes around $145^{\circ} \mathrm{C}$ or lower 
during cooling, irrespective of the chemical composition and the used OBOC concentration.
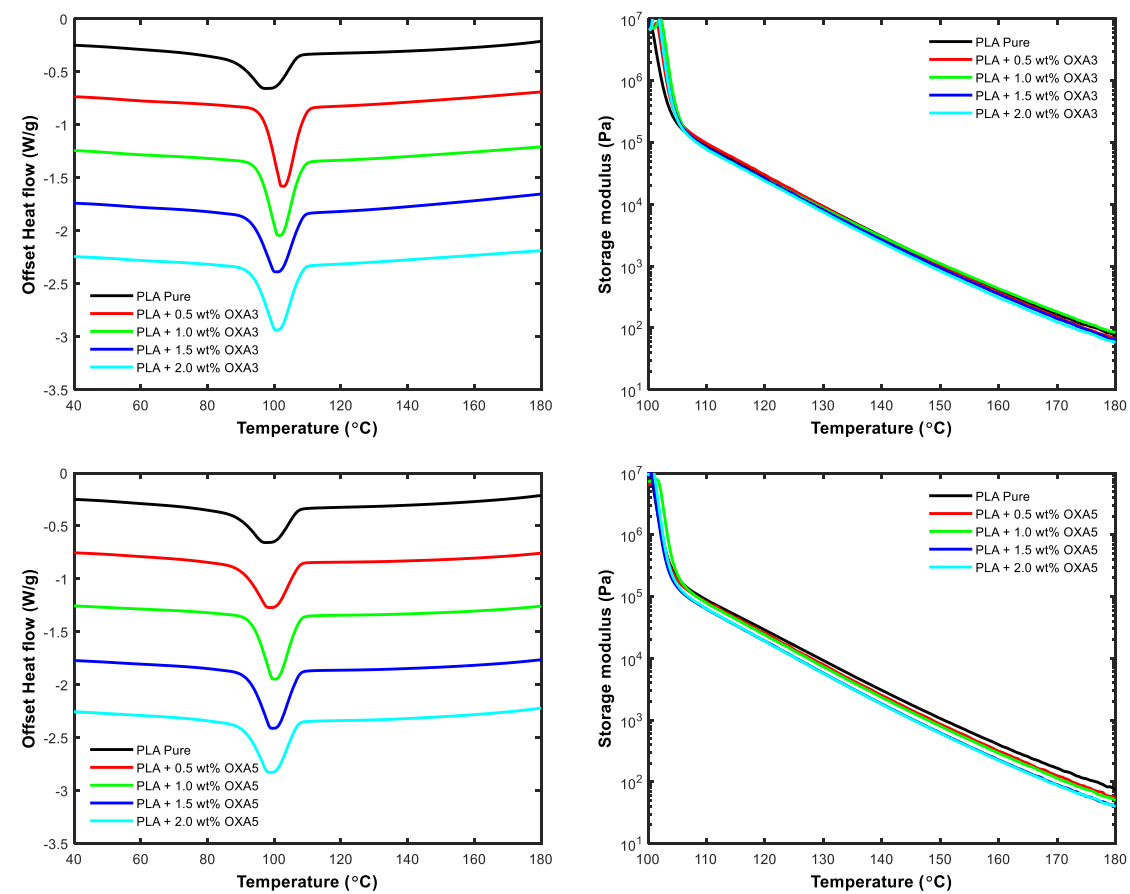

Figure 6.9. left, first DSC cooling traces taken at a cooling rate of $10{ }^{\circ} \mathrm{C} / \mathrm{min}$ for systems having varying concentration of OXA3 (top) and OXA5 (bottom), depicting the characteristic crystallization exotherms. Right, the evaluation of the storage modulus observed during cooling at a rate of $5{ }^{\circ} \mathrm{C} / \mathrm{min}$ for systems having varying OXA3 (top) and OXA5 (bottom) concentration. 

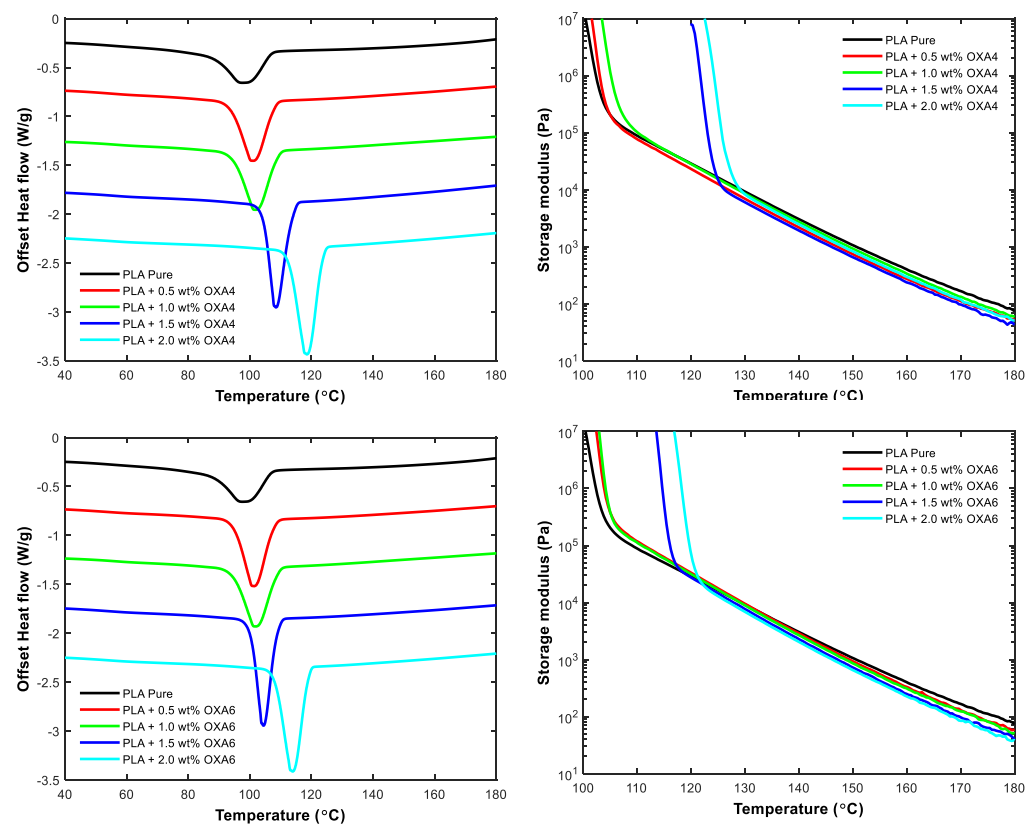

Figure 6.10. left, first DSC cooling traces taken at a cooling rate of $10{ }^{\circ} \mathrm{C} / \mathrm{min}$ for systems having varying concentration of OXA4 (top) and OXA6 (bottom), depicting the characteristic crystallization exotherms. Right, the evaluation of the storage modulus observed during cooling at a rate of $5{ }^{\circ} \mathrm{C} / \mathrm{min}$ for systems having varying OXA4 (top) and OXA6 (bottom) concentration.

\subsubsection{Identifying the OBOC nucleation mechanism}

To obtain more information on the nucleation mechanism, we have performed the following experiment in polarized optical microscopy (Figure 6.11): First, the sample containing $2.0 \mathrm{wt} \%$ OXA4 was heated to $200{ }^{\circ} \mathrm{C}$ to melt both the OXA4 and PLA crystals and to obtain a homogenous solution. Next, the sample was cooled to $145^{\circ} \mathrm{C}$ at a rate of $30^{\circ} \mathrm{C} / \mathrm{min}$ where it was kept under isothermal conditions for one minute to seed a low number of OXA4 crystals. Subsequently, the sample was heated at a rate of $30^{\circ} \mathrm{C} / \mathrm{min}$ to $155^{\circ} \mathrm{C}$ and maintained at this temperature for 10 minutes to stimulate the growth of the OXA4 crystals. Interestingly, at $155{ }^{\circ} \mathrm{C}$ the growth of a few PLA spherulites is observed on the center of the OXA4 crystals, likely corresponding to the growth of crystals that nucleated during the isotherm at $145{ }^{\circ} \mathrm{C}$ (micrographs B and C, Figure 6.11. After the 10 minute isotherm at $155{ }^{\circ} \mathrm{C}$, the sample is cooled at a rate of $10{ }^{\circ} \mathrm{C} / \mathrm{min}$ to $130{ }^{\circ} \mathrm{C}$, during which we observe 1) the further growth of existing OXA4 crystals, 2) the nucleation and growth of new OXA4 crystals, and 3) the nucleation and growth of PLA crystals. Interestingly, as is visible 
from the micrographs taken at $134^{\circ} \mathrm{C}$ and $130{ }^{\circ} \mathrm{C}$ (micrographs $\mathrm{E}$ and F, Figure 6.11), the formation of the transcrystalline PLA morphology is only observed on the 'freshly grown' OXA4 crystals parts; No PLA crystal growth is observed on the surface of the OXA4 crystals that were allowed to develop at $155^{\circ} \mathrm{C}$ (examples highlighted in micrograph F, Figure 6.11). Such behavior makes the possibility for both epitaxy and soft epitaxy as nucleation mechanisms by the OXA4 crystals highly unlikely. If these were the nucleating mechanisms, one would anticipate the nucleating efficiency to be solely dependent on the surface-to-volume ratio and the dispersion of the OBOC crystals, and independent on the temperature at which they were generated.

Instead, we would like to consider both local stresses imposed on the PLA melt by the growing OBOC crystals and their surface roughness as two alternative parameters that affect the PLA nucleation process. The concept of stress-enhanced nucleation has already been suggested as nucleation mechanism for the formation of the transcrystalline crystallization morphology in fiber reinforced thermoplastic polymers, where stress build-up occurs during cooling as a result of the mismatch in thermal expansion coefficient of the fiber and material matrix ${ }^{31-34}$.
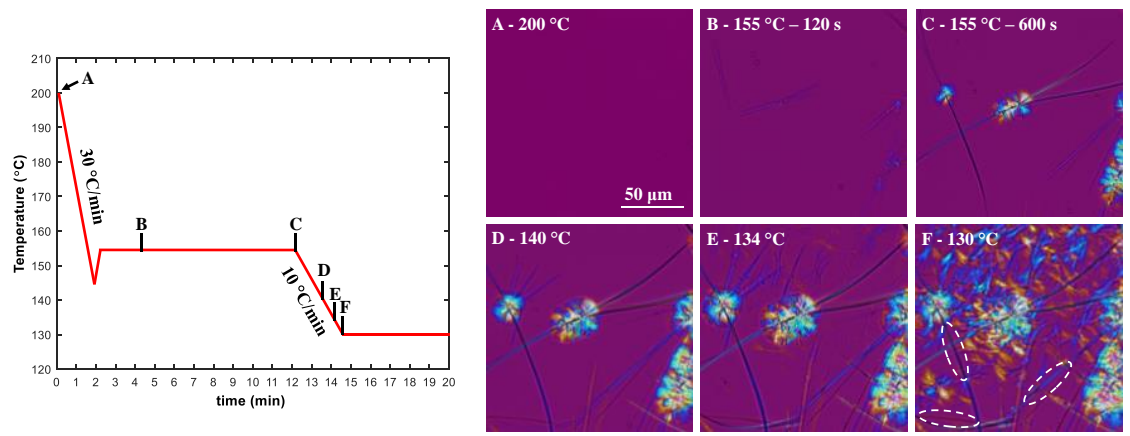

Figure 6.11. Left, Experimental protocol used to identify the effect of OXA4 crystallization temperature on the PLA nucleation process. Right, polarization optical micrographs depicting the morphological changes, according to the letters displayed in the experimental protocol.

In turn, the stresses imposed on the polymer melt can orient the contour of the polymer chains, a feature that is well known to lower the entropy of the chains and, hence, lower the nucleation barrier ${ }^{35-37}$. In our case, the growth of the OXA4 crystals will exert local stresses on the PLA melt in a similar manner, effectively imposing local shear on the PLA chains. The possibility for contour orientation, and hence stress-enhanced nucleation, can be evaluated by identification of the Weissenberg (Wi) number of this process. The dimensionless reptation or orientation $W i$ number is obtained by multiplying the imposed shear rate $\dot{\gamma}$ by the reptation time $\tau_{\text {rep }}$ and denotes the possibility for contour orientation of PLA chains when the value 
exceeds unity. In our experimental set-up, the local shear rate $\dot{\gamma}$ imposed on the PLA chains is approximated by taking the ratio of OXA4 crystal growth rate and the OXA4 radius at a given temperature or time ${ }^{38}$, whereas the reptation time $\tau_{\text {rep }}$ is obtained from rheology in combination with molecular modeling provided in detail in Appendix E (figures E1 and E2). The calculations used to determine both parameters are provided below.

\subsubsection{Approximation of shear rates during OBOC crystal growth}

To calculate the Weissenberg numbers (Wi) denoting the critical conditions for stress-enhanced nucleation or stress-induced crystallization, resulting respectively from chain orientation or stretch, we require both a value for the Rouse relaxation time and chain reptation time (shown in Appendix Figures E2c and E2d) and the shear rate imposed on the PLA melt by the growing OBOC crystals. To this end, the growth rate of the OXA4 crystals is assessed under two conditions: during cooling from elevated temperatures (shown in Figure 6.11), whereas an extended overview is provided in Figure 6.12) and under isothermal conditions at $140{ }^{\circ} \mathrm{C}$. The growth rate was determined from the OXA4 crystals highlighted by the white arrows in Figure 6.13. Please note, during the initial stage of crystal growth, we observe that the diameter is very small $(\sim 1 \mu \mathrm{m}$, Figure 6.12$)$ and hence difficult to observe in polarized optical microscopy. Furthermore, over time, the crystals grow both in length and in radius, indicating that they continuously impose stress to the PLA melt, and thus continuously impose local shear to the PLA chains residing close to the surface of the crystals.
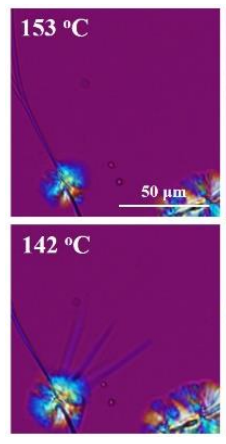

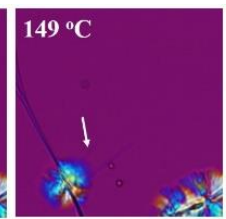

$140{ }^{\circ} \mathrm{C}$

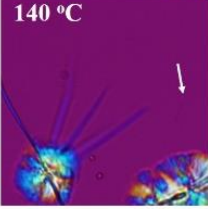

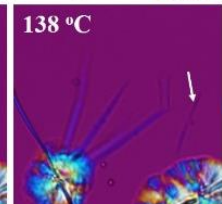
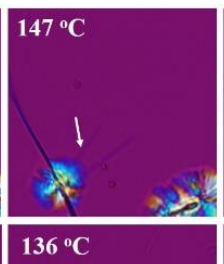

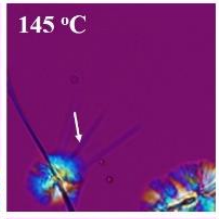

$134^{\circ} \mathrm{C}$

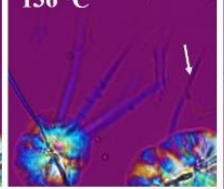

Figure 6.12. Polarization optical micrographs depicting the morphological changes when cooling the sample containing PLA and $2.0 \mathrm{wt} \%$ OXA4 from $155^{\circ} \mathrm{C}$ to $130{ }^{\circ} \mathrm{C}$ at a rate of $10^{\circ} \mathrm{C}$, during the following of the protocol outlined in Figure 6.2.

We approximate the shear rate $(\dot{\gamma})$ imposed on the PLA melt during the growth process of the tip of the OXA4 crystal by dividing the growth rate (in $\mu \mathrm{m} / \mathrm{s}$ ) by the radius of OXA4 crystal in the center. Note, the value of the radius in this 
calculation is likely overestimated for the growing crystals, as the OXA4 tip tapers towards the growth fronts, meaning that the actual shear rates in the system can be higher than those calculated. Nevertheless, we consider the used approximation adequate to verify whether the imposed shear rates are sufficiently high to facilitate molecular orientation or stretch on PLA chains. An overview of the calculated parameters of the OXA4 crystal growing between 149 and $138{ }^{\circ} \mathrm{C}$ is provided in Table 6.1.

Table 6.1. Overview of length $(L)$, radius $(R)$ and corresponding growth rate $(V)$, and corresponding shear rate $(\dot{\gamma})$ of OXA4 crystals during crystallization during cooling from the PLA melt (as observed from polarized optical microscopy depicted in Figure 6.12).

\begin{tabular}{|ccccc|}
$\begin{array}{c}\text { Temperature } \\
\left({ }^{\circ} \mathrm{C}\right)\end{array}$ & $\begin{array}{c}\mathrm{L}^{\mathrm{a}} \\
(\mu \mathrm{m})\end{array}$ & $\begin{array}{c}\mathrm{R} \\
(\mu \mathrm{m})\end{array}$ & $\begin{array}{c}\mathrm{V} \\
(\mu \mathrm{m} / \mathrm{s})\end{array}$ & $\begin{array}{c}\dot{\boldsymbol{\gamma}}^{\mathrm{b}} \\
\left(\mathrm{s}^{-1}\right)\end{array}$ \\
\hline $\mathbf{1 4 9}$ & 50.1 & 1.06 & - & - \\
\hline $\mathbf{1 4 7}$ & 54.7 & 1.33 & 1.16 & 0.88 \\
\hline $\mathbf{1 4 5}$ & 60.6 & 2.00 & 1.47 & 0.74 \\
\hline $\mathbf{1 4 2}$ & 66.9 & 2.52 & 1.58 & 0.62 \\
\hline $\mathbf{1 4 0}$ & 73.2 & 2.42 & 1.59 & 0.66 \\
\hline $\mathbf{1 3 8}$ & 80.1 & 2.36 & 1.70 & 0.72 \\
\hline
\end{tabular}

${ }^{a}$ As detected from polarized optical microscopy. ${ }^{a}$ pproximated by $V_{0} / R$.

The shear rate $\dot{\gamma}$ calculated in Table 6.1 in combination with the values for the relation times ( $\tau_{\mathrm{r}}$ and $\tau_{\text {rep }}$ ) presented in Figure E2c and E2d (Appendix) were used to calculate the Weissenberg numbers for molecular orientation and stretch displayed in Figure 6.13. As expected, and observed from Figure 6.13, the Rouse Wi numbers are several orders of magnitude too low to facilitate any contour or segmental stretch.

Additionally, shear rates applied by the growing OXA4 crystals are neither sufficient to facilitate a contour orientation when considering the whole molecular weight distribution. In contrast, when considering the $15 \%$ of the highest molecular weight chains, the main components generally involved in shear-induced crystallization, the reptation (orientation) $W i$ is roughly $1.8-2$ through the evaluated temperature range, confirming that these experimental conditions indeed favor stressenhanced nucleation. To obtain more information, OXA4 crystallites were allowed to grow under isothermal conditions at $140{ }^{\circ} \mathrm{C}$, as is shown in Figure 6.14. 

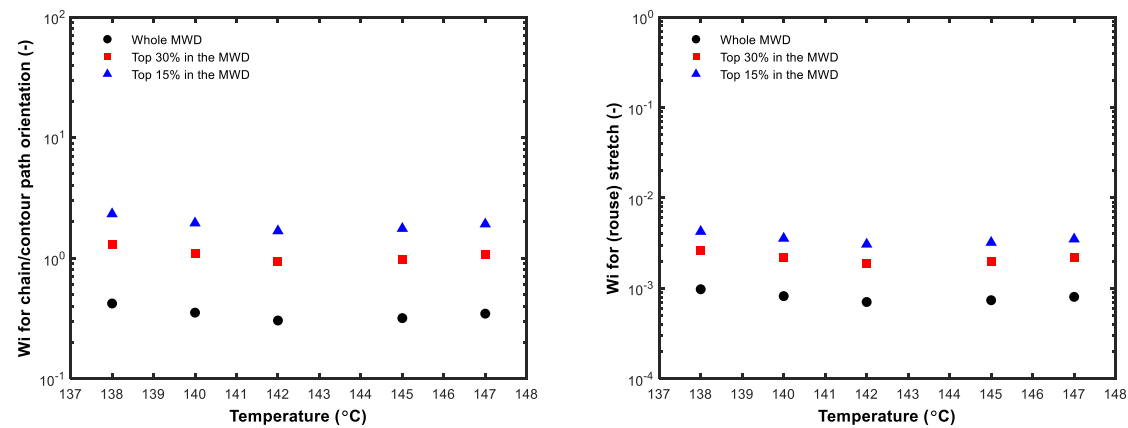

Figure 6.13. Estimated Weissenberg numbers (Wi) for contour orientation (left) and (rouse) stretch (right) of the PLA chains, as a result of the OXA4 crystal growth depicted in Figure 6.13.

PLA + 2.0 wt \% OXA4 - Isothermal at $140{ }^{\circ} \mathrm{C}$
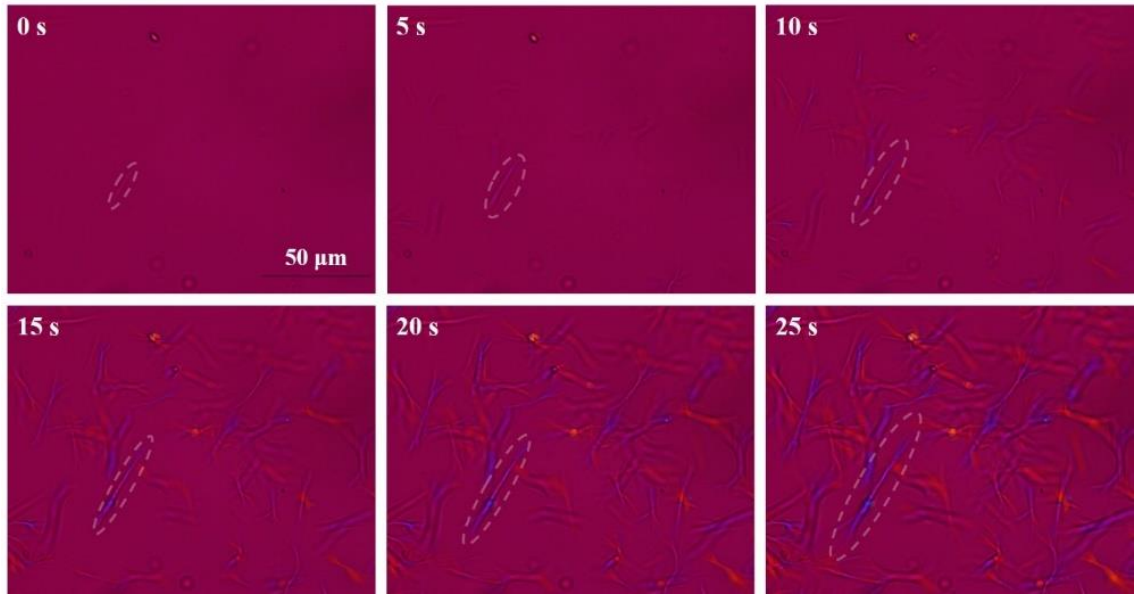

Figure 6.14. Polarization optical micrographs depicting the morphological changes of the PLA sample containing PLA and $2.0 \mathrm{wt} \%$ OXA4 under isothermal conditions at $140{ }^{\circ} \mathrm{C}$, after cooling the sample from $200{ }^{\circ} \mathrm{C}$ to $140{ }^{\circ} \mathrm{C}$ at a rate of $30{ }^{\circ} \mathrm{C} / \mathrm{min}$. Note, to ease the monitoring of the growth of the OXA4 crystals, transparent white circles are drawn around the crystals used to calculate the growth rate.

When determining the growth in both length and diameter of the OXA4 crystal (Figure 6.15) over time we observe that the crystal grows at a rate of approximately $1 \mu \mathrm{m} / \mathrm{s}$ (the length increases roughly at a rate of $10 \mu \mathrm{m} / 5$ seconds, where the crystal has two growing fronts). Similarly, the diameter of the crystal increases slowly from $1.2 \mu \mathrm{m}$ to $2.9 \mu \mathrm{m}$ during the crystallization process, translating to shear rates varying between 0.6 and $1.2 \mathrm{~s}^{-1}$. 


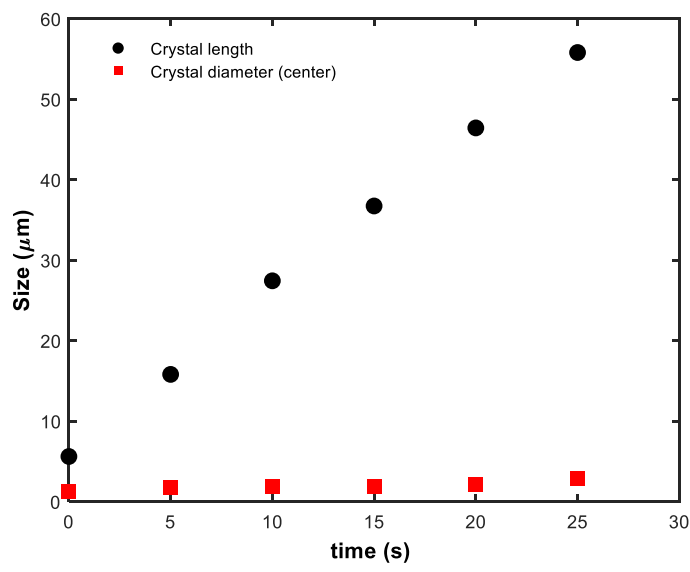

Figure 6.15. Crystal length and diameter determined from the OXA4 crystal growing under isothermal conditions at $140^{\circ} \mathrm{C}$, as depicted in Figure 6.14.

These values correspond to an orientation $W i$ varying between $0.3-0.6$ (considering the whole MWD), 1 - 2 (considering the $30 \%$ highest molecular weight chains), and $1.8-3.6$ (considering the $15 \%$ highest molecular weight chains). Again, this experiment suggests that stress-enhanced nucleation is plausible to proceed under the experimental conditions.

Notice that the Rouse Weissenberg numbers were estimated as well, though they are considerably lower than unity suggesting that rouse stretch is not realized under the experimental conditions of the current study. Instead, according to the calculations presented above during isothermal OXA4 crystallization at $140{ }^{\circ} \mathrm{C}$, we observe that the growing OXA4 crystals can facilitate local shear rates of 0.6 to 1.2 $\mathrm{s}^{-1}$ on the PLA melt. This corresponds to $W_{i}$ numbers for contour orientation of PLA chains between $0.3-0.6$ (considering the whole molecular weight distribution) up to 1.8 - 3.6 (considering the $15 \%$ highest molecular weight chains). In other words, this approximation suggests that in particular the contour of the high molecular weight PLA chains orients by the shear rates imposed by the growing OXA4 crystals. Furthermore, these findings suggest that stress-enhanced nucleation is possible under the experimental conditions in this study.

To reflect, Iqbal and coworkers demonstrated in a recent study that shearenhanced nucleation indeed proceeds when exposing the PLA to shear rates as low as $0.1 \mathrm{~s}^{-1}$ for 10 seconds at $140{ }^{\circ} \mathrm{C}^{39}$. However, though an enhancement in nucleation rate was observed compared to the pure PLA reference, Iqbal and coworkers observed an induction time of several minutes before crystallization proceeds after the application of the shear pulse. Furthermore, increasing the shear rate to $\dot{\gamma}=2.0 \mathrm{~s}^{-1}$ 
(10 seconds pulse) still resulted in an induction time of more than 100 seconds before crystallization was observed. Based on these findings, despite the continuous increase in both OXA4 crystal length and diameter, which effectively imposes a continuous stress on the PLA chains close to the growing OXA4 crystals, we would expect longer induction times for PLA nucleation and crystal growth than those observed in our experiments.

In addition, surface roughness, a parameter that is widely considered to influence the heterogeneous nucleation process ${ }^{40-45}$, is likely to enhance the nucleation process of PLA in the presence of the growing OXA4 crystal even further. The argumentation is as follows: Freshly grown OXA4 crystal parts are likely to contain defects such as dislocations, potentially generating crystals with a high surface roughness. Following the findings depicted in Figure 6.12, the OXA4 crystals grown at $155{ }^{\circ} \mathrm{C}$ do not facilitate heterogeneous nucleation of the PLA matrix, indicating that they might contain less defects and hence a lowered surface roughness. The origin of this difference in surface roughness can be two-fold. First, the 'freshly grown' OXA4 crystal segments contain a high number of defects, which reorganize and/or recrystallize during the isothermal annealing step at $155^{\circ} \mathrm{C}$. Second, we consider it likely that crystals formed at elevated temperatures are already less defected resulting from the lower supersaturation of the system, which decreases both the crystal growth rate and the chance of defect formation. Either way, the OXA4 crystals generated at or exposed to elevated temperatures are expected to exhibit low surface roughness, decreasing their capability to promote heterogeneous nucleation of PLA.

To verify these findings from polarized optical microscopy and support the above mentioned hypotheses, isothermal crystallization studies were performed and subjected to an Avrami analysis ${ }^{28-30}$. To this end, PLA samples with and without 2.0 wt $\%$ OXA2 were heated to $190{ }^{\circ} \mathrm{C}$ and kept isothermal for 3 minutes to ensure both the PLA and the OXA2 are molten and to erase the thermal history of the sample. The sample was cooled at a rate of $50{ }^{\circ} \mathrm{C} / \mathrm{min}$ to a first isothermal (holding) temperature between 145 and $170{ }^{\circ} \mathrm{C}$ for 5 minutes to allow for the generation of OXA2 crystals. Next, the sample was cooled at a rate of $50{ }^{\circ} \mathrm{C} / \mathrm{min}$ to $140{ }^{\circ} \mathrm{C}$ after which the isothermal crystallization of the PLA was monitored (Figure 6.16A). As reference, the isothermal crystallization for both pure PLA and PLA with $2.0 \mathrm{wt} \%$ OXA2 was monitored after cooling to $140{ }^{\circ} \mathrm{C}$ directly (black and red lines in Figure 6.16 , respectively). The isothermal crystallization exotherms displayed in Figure 6.16B clearly demonstrate that the PLA reference (black line) crystallizes particularly slowly at $140{ }^{\circ} \mathrm{C}$, with a half-time of crystallization $\left(\mathrm{t}_{\text {half }}-\mathrm{t}_{0}\right)$ of 26.5 minutes. In contrast, the presence of $2.0 \mathrm{wt} \%$ OXA 2 decreases $t_{\text {half }}$ - $\mathrm{t}_{0}$ significantly to 4.0 when subjecting the sample to the same experimental protocol (red line, Figure 6.16B). 
Unfortunately, the samples subjected to a holding temperature of $145^{\circ} \mathrm{C}$ and $150{ }^{\circ} \mathrm{C}$ started to crystallize already at the holding temperature, preventing us from obtaining a useable crystallization exotherm upon cooling to $140^{\circ} \mathrm{C}$ and are therefore excluded from further analysis.
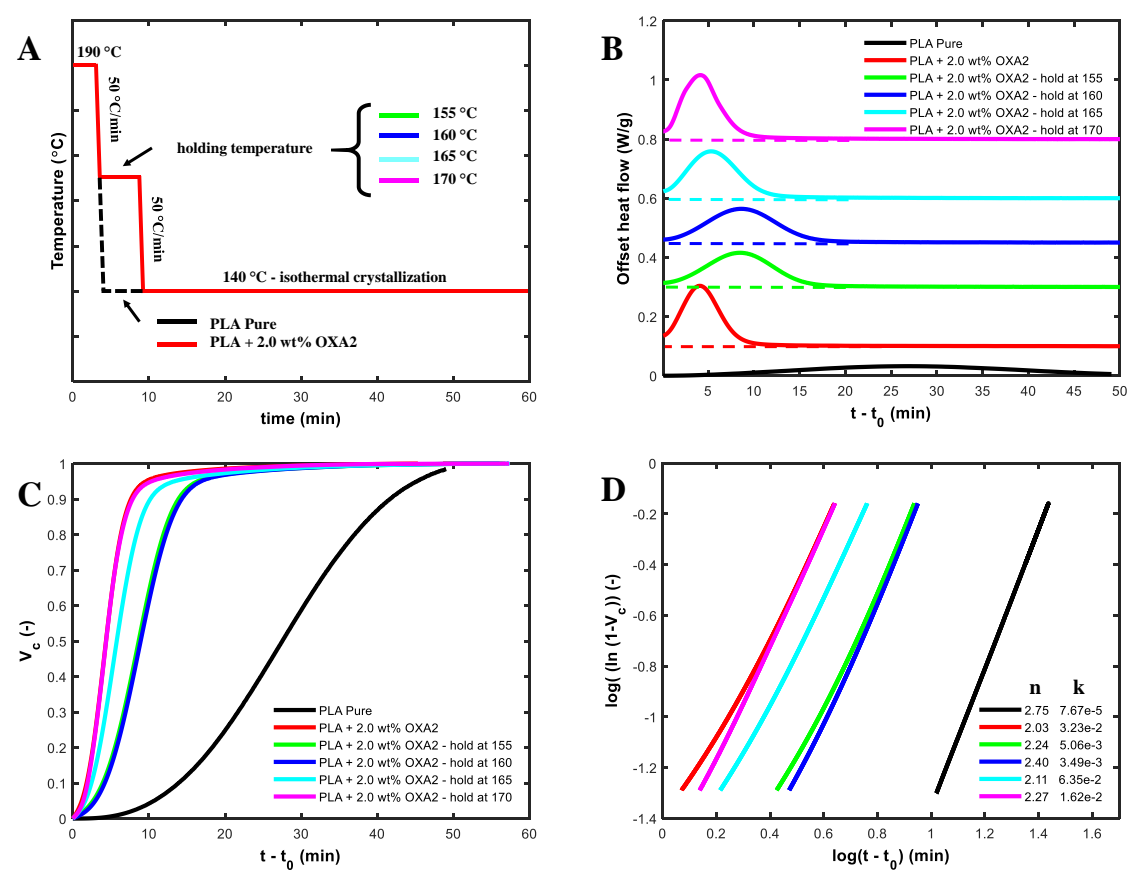

Figure 6.16. A) Schematic overview of experimental protocol employed to assess the effect the influence of OXA2 crystallization temperature on the isothermal crystallization of PLA at $140{ }^{\circ} \mathrm{C}$. B) Isothermal crystallization isotherms (exo up) obtained at $140{ }^{\circ} \mathrm{C}$ following the experimental protocol displayed in image A). C) Development of the relative volumetric crystalline fraction $\left(V_{c}\right)$ during isothermal crystallization at $140{ }^{\circ} \mathrm{C}$. D) Overview of Avrami plots taking into account the development of $V_{c}$ between 5 and $50 \%\left(k\right.$ in $\left.\mathrm{min}^{-n}\right)$.

However, as expected, the isothermal crystallization process of PLA with $2.0 \mathrm{wt} \% \mathrm{OXA} 2$ at $140{ }^{\circ} \mathrm{C}$ is slowed down for samples which were subjected to a first isothermal temperature between $155^{\circ} \mathrm{C}$ and $160{ }^{\circ} \mathrm{C}\left(\mathrm{t}_{\text {half }} \mathrm{-}_{0}\right.$ of 8.5 and 8.6 minutes, respectively). As can be deduced from Figure 6.5, only a part of the dissolved OXA2 molecules is taken up in the OXA2 crystals growing during the isothermal holding temperature, yielding crystals that are unable to promote heterogeneous nucleation upon further cooling to $140{ }^{\circ} \mathrm{C}$. Instead, the enhancement in nucleation efficiency compared to the pure PLA sample originates from the remainder of the dissolved 
OXA2 molecules that crystallize during/after cooling to $140{ }^{\circ} \mathrm{C}$ and effectively facilitate the growth of a transcrystalline morphology. However, a lower number of heterogeneous nucleation events can be expected as a result of the presence of 'inert' OXA2 crystals (i.e. without the ability to facilitate heterogeneous nucleation of PLA crystals), translating into a slower increase of the volumetric crystalline fraction $\left(V_{\mathrm{c}}\right)$ over time when compared to the same sample immediately cooled to $140{ }^{\circ} \mathrm{C}$ (Figure 6.16C). This effect diminishes for samples subjected to an isothermal holding temperature of 165 or $170{ }^{\circ} \mathrm{C}\left(\mathrm{t}_{\text {alf }} \mathrm{t}_{0}\right.$ of 5.2 and 4.1 minutes, respectively), as the rate of OXA2 crystallization at this holding temperature is very low. Accordingly, a high OXA2 concentration remains dissolved at the holding temperature and only crystallizes during cooling to or upon reaching $140{ }^{\circ} \mathrm{C}$, effectively yielding a scenario very similar to the system which was directly cooled to $140{ }^{\circ} \mathrm{C}$.

The difference in nucleation density in the different systems becomes evident when comparing the Avrami plots of the different samples (Figure 6.16D). For example, for the pure PLA reference (black line) we observe $n=2.75$, a value significantly larger than for the PLA with $2.0 \mathrm{wt} \%$ OXA2 reference (red line), which yields $n=2.03$. Classically, an Avrami parameter $n$ of 2 would suggest the growth of 2-dimensional crystals and the occurrence of instantaneous nucleation ${ }^{43}$, two features which can be expected in the presence of OXA2 crystals as they stimulate transcrystalline PLA crystal growth under the right conditions (Figure 6.5). The value of $n=2.75$ for the pure PLA reference is close to the values reported in literature (varying from $2.4-3.3)^{29,46}$ and is generally attributed to athermal and threedimensional crystal growth. When subjecting the PLA with $2.0 \mathrm{wt} \%$ OXA2 to an isothermal holding time at $155^{\circ} \mathrm{C}$ or $160{ }^{\circ} \mathrm{C}$ prior to isothermal crystallization at 140 ${ }^{\circ} \mathrm{C}$, the Avrami parameter $\mathrm{n}$ slowly increases to 2.24 and 2.40 , respectively, suggesting a decrease in nucleation rate and/or a change in PLA crystal morphology. As explained above, such a change in nucleation and crystallization behavior is expected as part of the OXA2 is depleted during the isothermal holding temperature and is unable to facilitate heterogeneous nucleation of the PLA upon consecutive cooling. As expected, when increasing the holding temperature further, the Avrami parameter $n$ decreases again to 2.11 and 2.27 (holding temperatures of $165^{\circ} \mathrm{C}$ and $170{ }^{\circ} \mathrm{C}$, respectively). We would like to highlight that one should consider the obtained Avrami parameters as qualitative given that the nucleation and crystallization processes in these samples are rather complex combined with the fact that crystallization proceeds in some samples already during cooling towards $140{ }^{\circ} \mathrm{C}$ (Figure 6.17B). Nevertheless, these findings depict a clear trend in crystallization behavior that supports the observation that OXA crystals grown at elevated temperatures are unable to provide surface for heterogeneous nucleation of PLA, and hence, result in a decrease in nucleating efficiency of this class of nucleating agents. 


\subsubsection{Optimizing processing conditions for improved PLA nucleation}

Retrospectively, we learned that the OBOC crystal growth should proceed at temperatures around $150-145^{\circ} \mathrm{C}$ or lower for the formation of a transcrystalline PLA morphology on the OBOC crystal surface. The critical parameter that drives the OBOC nucleation and crystal growth rate is the supersaturation, which is governed by the pure OBOC melting temperature, the employed OBOC concentration, and the imposed cooling rate ${ }^{4-50}$. In the previous section, we have elaborated on the interplay between the OBOC melting temperature, concentration, and thermal history on the PLA crystallization morphology (Figure 6.7), leaving the cooling rate as an additional parameter. When subjecting the samples to increasing cooling rates, a higher supersaturation can be achieved before the OBOC crystallization proceeds from the PLA melt. As both the OBOC nucleation and growth rate are generally expected to increase with increasing supersaturation ${ }^{43}$, the OBOC will crystallize with an increasing surface-to-volume ratio, hence there will be more surface available for heterogeneous nucleation of PLA ${ }^{24,25}$. In addition, increased OBOC growth rates will increase both the local stresses imposed on the PLA melt and the defects and surface roughness of the generated OBOC crystals. For example, as described in the previous sections, during cooling at a rate of $10{ }^{\circ} \mathrm{C} / \mathrm{min}$, the OXA2 is not effective in generating a transcrystalline PLA morphology when used in a concentration of 2.0 wt\%, while the OXA4 and OXA6 systems are. However, when subjecting these same samples to a cooling rate of $50{ }^{\circ} \mathrm{C} / \mathrm{min}$, one can observe the $\mathrm{OXA} 2$ becomes the most efficient nucleating agent when monitoring the isothermal crystallization at $135^{\circ} \mathrm{C}$ (Figure 6.17, left).

For example, the crystallization half-time $\left(\mathrm{t}_{\text {half }}-\mathrm{t}_{0}\right)$ observed under these conditions is 2.7 minutes, 8.6 minutes and more than 30 minutes for the systems containing 2 wt $\%$ OXA2, OXA4, and OXA6 respectively. For comparison, pure PLA displays a $t_{\text {half }}-\mathrm{t}_{0}$ of 17.7 minutes, indicating that the presence of $2.0 \mathrm{wt} \%$ OXA6 in fact delays the nucleation and crystallization process under these conditions. Such a delay in nucleation and crystallization rate is a characteristic feature of a plasticizer; Indeed, as observed from Figure 6.6, OXA6 remains dissolved at this temperature and could indeed act as a plasticizer, as we have reported for OBOCs dissolved in isotactic polypropylene ${ }^{24,51}$. When performing the same isothermal crystallization experiments for the PLA containing $2.0 \mathrm{wt} \%$ OXA2, but at different isothermal temperatures (Figure 6.17, right) we observe that PLA crystallization proceeds rapidly at $145{ }^{\circ} \mathrm{C}$ and lower, in line with the previous findings. However, the crystallization half-time $\left(\mathrm{t}_{\text {half }}-\mathrm{t}_{0}\right)$ increases rapidly during isothermal crystallization at $150{ }^{\circ} \mathrm{C}$, suggesting that the OXA2 crystal growth proceeds sufficiently slow to 1) allow for the PLA phase to relax all imposed stresses and 2) develop crystals with a low surface roughness. Note, the decrease in PLA crystal growth rate as a function of 
the decreasing undercooling of the PLA melt should be taken into account. Nevertheless, the findings displayed in Figures 6.16 and 6.17 confirm that a high cooling rate and the selection of a low crystallization temperature $\left(\leq 145{ }^{\circ} \mathrm{C}\right)$ are essential for an optimal OBOC nucleation efficiency. These features are favorable for use under industrial processing conditions, as they generally require high cooling rates.
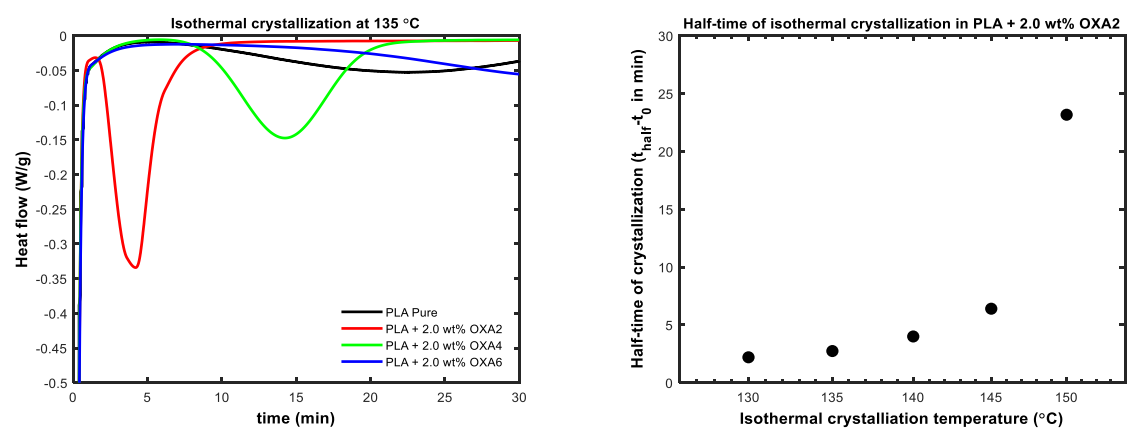

Figure 6.17. Left, DSC thermograms depicting the crystallization exotherm observed during isothermal conditions (at $135^{\circ} \mathrm{C}$ ) after cooling pure PLA, and PLA with 2.0 wt\% OXA2, OXA4, and OXA6 from the melt at a rate of $100{ }^{\circ} \mathrm{C} / \mathrm{min}$ to $135^{\circ} \mathrm{C}$. Right, half-time of crystallization ( $t_{\text {half }}-t_{0}$ ) as a function of the isothermal crystallization temperature in PLA systems containing 2.0 wt\% OXA2, observed from DSC experiments after cooling from $200{ }^{\circ} \mathrm{C}$ to the desired isothermal crystallization temperature at a rate of $50{ }^{\circ} \mathrm{C} / \mathrm{min}$.

\subsection{Conclusions}

In this work, we synthesized five different melt-soluble oxalamide based organic compounds (OBOCs) and evaluated their nucleation mechanism for PLA. The OBOCs indeed dissolve at elevated temperatures in the PLA melt and, dependent on the pure OBOC melting temperature and the used concentration, crystallizes upon cooling after which they provide a surface for heterogeneous nucleation to PLA. However, the effective nucleation efficiency seems highly dependent on the crystallization temperature of the OBOC from the PLA melt. These findings indicate that epitaxy or soft-epitaxy are unlikely nucleation mechanisms for these OBOCs. Instead, we identify surface roughness of the OBOC crystals in combination with local stresses imposed on the PLA melt by the growing OBOC crystals as critical parameters to explain this variation in nucleation efficiency. Since both surface roughness and OBOC crystal growth rates increase with increasing supersaturation, the use of OBOCs as nucleating agents is identified to be more suitable in processes where high cooling rates are desired. In this respect, the role of OBOCs is rather unique compared to the reported nucleating agents for PLAs in the literature. 


\section{For more information, please refer Appendix E.}

\subsection{References}

(1) Kwon, S.; Lee, P. C.; Lee, E. G.; Keun Chang, Y.; Chang, N. Production of Lactic Acid by Lactobacillus Rhamnosus with Vitamin- Supplemented Soybean Hydrolysate. Enzyme Microb. Technol. 2000, 26 (2-4), 209-215. https://doi.org/10.1016/S0141-0229(99)00134-9.

(2) Gao, C.; Ma, C.; Xu, P. Biotechnological Routes Based on Lactic Acid Production from Biomass. Biotechnol. Adv. 2011, 29 (6), 930-939. https://doi.org/10.1016/j.biotechadv.2011.07.022.

(3) Bilanovic, D.; Chang, F. H.; Isobaev, P.; Welle, P. Lactic Acid and Xanthan Fermentations on an Alternative Potato Residues Media - Carbon Source Costs. Biomass and Bioenergy 2011, 35 (7), 2683-2689. https://doi.org/10.1016/j.biombioe.2011.03.001.

(4) Fukushima, K.; Sogo, K.; Miura, S.; Kimura, Y. Production of D -Lactic Acid by Bacterial Fermentation of Rice Starch. 2004, 1021-1027. https://doi.org/10.1002/mabi.200400080.

(5) Leal, C. V.; dos Santos Almeida, R.; Dávila, J. L.; Domingues, J. A.; Hausen, M. A.; Duek, E. A. R.; d'Ávila, M. A. Characterization and in Vitro Evaluation of Electrospun Aligned-Fiber Membranes of Poly(L-Co-D,L-Lactic Acid). J. Appl. Polym. Sci. 2019, 47657, 1-9. https://doi.org/10.1002/app.47657.

(6) Bertuoli, P. T.; Ordono, J.; Armelin, E.; Pérez-Amodio, S.; Baldissera, A. F.; Ferreira, C. A.; Puiggalí, J.; Engel, E.; Del Valle, L. J.; Alemán, C. Electrospun Conducting and Biocompatible Uniaxial and Core-Shell Fibers Having Poly(Lactic Acid), Poly(Ethylene Glycol), and Polyaniline for Cardiac Tissue Engineering. ACS Omega 2019, 4 (2), 3660-3672. https://doi.org/10.1021/acsomega.8b03411.

(7) Frackowiak, S.; Ludwiczak, J.; Leluk, K. Man-Made and Natural Fibres as a Reinforcement in Fully Biodegradable Polymer Composites: A Concise Study. J. Polym. Environ. 2018, 26 (12), 4360-4368. https://doi.org/10.1007/s10924-018-1301-9.

(8) Morreale, M.; Mistretta, M. C.; Fiore, V. Creep Behavior of Poly(Lactic Acid) Based Biocomposites. Materials (Basel). 2017, 10 (4). https://doi.org/10.3390/ma10040395.

(9) Tábi, T.; Bakonyi, P.; Hajba, S.; Herrera-Franco, P. J.; Czigány, T.; Kovács, J. G. Creep Behaviour of Injection-Moulded Basalt Fibre Reinforced Poly(Lactic Acid) Composites. $J$. Reinf. Plast. Compos. 2016, 35 (21), 1600-1610. https://doi.org/10.1177/0731684416661239.

(10) Santis, F. De; Volpe, V.; Pantani, R. Effect of Molding Conditions on Crystallization Kinetics and Mechanical Properties of Poly ( Lactic Acid ). 2017, 1-6. https://doi.org/10.1002/pen.

(11) Xu, J. Z.; Zhang, Z. J.; Xu, H.; Chen, J. Bin; Ran, R.; Li, Z. M. Highly Enhanced Crystallization Kinetics of Poly(1 -Lactic Acid) by Poly(Ethylene Glycol) Grafted Graphene Oxide Simultaneously as Heterogeneous Nucleation Agent and Chain Mobility Promoter. Macromolecules 2015, 48 (14), 4891-4900. https://doi.org/10.1021/acs.macromol.5b00462.

(12) Xie, Q.; Han, L.; Shan, G.; Bao, Y.; Pan, P. Polymorphic Crystalline Structure and Crystal Morphology of Enantiomeric Poly(Lactic Acid) Blends Tailored by a Self-Assemblable Aryl Amide Nucleator. ACS Sustain. Chem. Eng. 2016, 4 (5), 2680-2688. 
https://doi.org/10.1021/acssuschemeng.6b00191.

(13) Yin, H. Y.; Wei, X. F.; Bao, R. Y.; Dong, Q. X.; Liu, Z. Y.; Yang, W.; Xie, B. H.; Yang, M. B. High-Melting-Point Crystals of Poly(1-Lactic Acid) (PLLA): The Most Efficient Nucleating Agent to Enhance the Crystallization of PLLA. CrystEng Comm 2015, 17 (11), 2310-2320. https://doi.org/10.1039/c4ce02497d.

(14) Liang, J. Z.; Zhou, L.; Tang, C. Y.; Tsui, C. P. Crystalline Properties of Poly(L-Lactic Acid) Composites Filled with Nanometer Calcium Carbonate. Compos. Part B Eng. 2013, 45 (1), 1646-1650. https://doi.org/10.1016/j.compositesb.2012.09.086.

(15) Pei, A.; Zhou, Q.; Berglund, L. A. Functionalized Cellulose Nanocrystals as Biobased Nucleation Agents in Poly ( L -Lactide ) ( PLLA ) - Crystallization and Mechanical Property Effects. 2010, 70, 815-821. https://doi.org/10.1016/j.compscitech.2010.01.018.

(16) Neppalli, R.; Causin, V.; Marega, C.; Modesti, M.; Adhikari, R.; Scholtyssek, S.; Ray, S. S.; Marigo, A. The Effect of Different Clays on the Structure, Morphology and Degradation Behavior of Poly(Lactic Acid). Appl. Clay Sci. 2014, 87, 278-284. https://doi.org/10.1016/j.clay.2013.11.029.

(17) De Santis, F.; Pantani, R. Melt Compounding of Poly (Lactic Acid) and Talc: Assessment of Material Behavior during Processing and Resulting Crystallization. J. Polym. Res. 2015, 22 (12), 1-9. https://doi.org/10.1007/s10965-015-0885-1.

(18) Gupta, A.; Simmons, W.; Schueneman, G. T.; Mintz, E. A. Lignin-Coated Cellulose Nanocrystals as Promising Nucleating Agent for Poly(Lactic Acid). J. Therm. Anal. Calorim. 2016, 126 (3), 1243-1251. https://doi.org/10.1007/s10973-016-5657-6.

(19) Fan, Y.; Zhu, J.; Yan, S.; Chen, X.; Yin, J. Nucleating Effect and Crystal Morphology Controlling Based on Binary Phase Behavior between Organic Nucleating Agent and Poly(1Lactic Acid). Polymer (Guildf). 2015, 67, 63-71. https://doi.org/10.1016/j.polymer.2015.04.062.

(20) Xu, T.; Zhang, A.; Zhao, Y.; Han, Z.; Xue, L. Crystallization Kinetics and Morphology of Biodegradable Poly(Lactic Acid) with a Hydrazide Nucleating Agent. Polym. Test. 2015, 45, 101-106. https://doi.org/10.1016/j.polymertesting.2015.05.009.

(21) Carbone, M. J.; Vanhalle, M.; Goderis, B.; Van Puyvelde, P. Amino Acids and Poly(Amino Acids) as Nucleating Agents for Poly(Lactic Acid). J. Polym. Eng. 2015, 35 (2), 169-180. https://doi.org/10.1515/polyeng-2014-0175.

(22) Xu, Y.; Wang, D.; Dong, W.; Chen, M.; Ma, P.; Xu, Y.; Wang, D.; Dong, W.; Chen, M.; Ma, P.; et al. Rapid Crystallization of Poly(Lactic Acid) by Using Tailor-Made Oxalamide Derivatives as Novel Soluble-Type Nucleating Agents. Ind. Eng. Chem. Res. 2014, 53 (32), 12888-12892. https://doi.org/10.1021/ie502211j.

(23) Ma, P.; Deshmukh, Y. S.; Wilsens, C. H. R. M.; Ryan Hansen, M.; Graf, R.; Rastogi, S. SelfAssembling Process of Oxalamide Compounds and Their Nucleation Efficiency in BioDegradable Poly(Hydroxyalkanoate)S. Sci. Rep. 2015, 5 (February), 1-9. https://doi.org/10.1038/srep13280.

(24) Wilsens, C. H. R. M.; Hawke, L. G. D.; Troisi, E. M.; Hermida-Merino, D.; De Kort, G.; Leoné, N.; Saralidze, K.; Peters, G. W. M.; Rastogi, S. Effect of Self-Assembly of Oxalamide 
Based Organic Compounds on Melt Behavior, Nucleation, and Crystallization of Isotactic Polypropylene. Macromolecules 2018, 51 (13), 4882-4895.

https://doi.org/10.1021/acs.macromol.8b00489.

(25) Deshmukh, Y. S.; Wilsens, C. H. R. M.; Leone, N.; Portale, G.; Harings, J. A. W.; Rastogi, S. Melt-Miscible Oxalamide Based Nucleating Agents and Their Nucleation Efficiency in Isotactic Polypropylene. Ind. Eng. Chem. Res. 2016, 55 (45), 11756-11766.

https://doi.org/10.1021/acs.iecr.6b03120.

(26) Yang, J.; Liang, R.; Kong, R.; Chen, Y.; Wang, X. X.; Yin, J.; Wan, J.; Wang, X. X.; Bi, C. Crystal Morphology, Crystallization Behavior, Polymorphic Crystalline Structure and Thermal Stability of Poly(1,4-Butylene Adipate) Modulated by a Oxalamide Derivative Nucleating Agent. Polym. Degrad. Stab. 2017, 144, 33-42.

https://doi.org/10.1016/j.polymdegradstab.2017.07.030.

(27) Yang, J.; Liang, R.; Chen, Y.; Zhang, C.; Zhang, R.; Wang, X.; Kong, R.; Chen, Q. Using a Self-Assemblable Nucleating Agent to Tailor Crystallization Behavior, Crystal Morphology, Polymorphic Crystalline Structure, and Biodegradability of Poly(1,4-Butylene Adipate). Ind. Eng. Chem. Res. 2017, 56 (28), 7910-7919. https://doi.org/10.1021/acs.iecr.7b01783.

(28) Avrami, M. Kinetics of Phase Change. II Transformation-Time Relations for Random Distribution of Nuclei. J. Chem. Phys. 1940, 8 (2), 212-224. https://doi.org/10.1063/1.1750631.

(29) Miyata, T.; Masuko, T. Crystallization Behaviour of Poly(L-Lactide). Polymer (Guildf). 1998, 39 (22), 5515-5521. https://doi.org/10.1016/S0032-3861(97)10203-8.

(30) Lorenzo, A. T.; Arnal, M. L.; Albuerne, J.; Müller, A. J. DSC Isothermal Polymer Crystallization Kinetics Measurements and the Use of the Avrami Equation to Fit the Data: Guidelines to Avoid Common Problems. Polym. Test. 2007, 26 (2), 222-231. https://doi.org/10.1016/j.polymertesting.2006.10.005.

(31) Thomason, J. L.; Van Rooyen, A. A. Transcrystallized Interphase in Thermoplastic Composites - Part II Influence of Interfacial Stress, Cooling Rate, Fibre Properties and Polymer Molecular Weight. J. Mater. Sci. 1992, 27 (4), 897-907. https://doi.org/10.1007/BF01197639.

(32) Gray, D. G. "Transcrystallization" Induced by Mechanical Stress on a Polypropylene Melt. J. Polym. Sci. Polym. Lett. Ed. 1974, 12 (11), 645-650. https://doi.org/10.1002/pol.1974.130121107.

(33) Wang, C.; Liu, C. R. Transcrystallization of Polypropylene on Carbon Fibres. Polymer (Guildf). 1997, 38 (18), 4715-4718. https://doi.org/10.1016/S0032-3861(96)01083-X.

(34) Wang, C.; Liu, C. R. Transcrystallization of Polypropylene Composites: Nucleating Ability of Fibres. Polymer (Guildf). 1999, 40 (2), 289-298. https://doi.org/10.1016/S00323861(98)00240-7.

(35) Meerveld, J. Van. Towards a Rheological Classification of Flow Induced Crystallization Experiments of Polymer Melts. 2004, 119-134. https://doi.org/10.1007/s00397-004-0382-7.

(36) Acierno, S.; Palomba, B.; Winter, H. H.; Grizzuti, N. Effect of Molecular Weight on the FlowInduced Crystallization of Isotactic Poly(1-Butene). Rheol. Acta 2003, 42 (3), 243-250. https://doi.org/10.1007/s00397-002-0280-9. 
(37) Coppola, S.; Balzano, L.; Gioffredi, E.; Maffettone, P. L.; Grizzuti, N. Effects of the Degree of Undercooling on Flow Induced Crystallization in Polymer Melts. Polymer (Guildf). 2004, 45 (10), 3249-3256. https://doi.org/10.1016/j.polymer.2004.03.049.

(38) Ahonguio, F.; Jossic, L.; Magnin, A. Motion and Stability of Cones in a Yield Stress Fluid. AIChE J. 2015, 61 (2), 709-717. https://doi.org/10.1002/aic.14651.

(39) Iqbal, N.; Jariyavidyanont, K.; Rhoades, A. M.; Androsch, R. Critical Specific Work of Flow for Shear-induced Formation of Crystal Nuclei in Poly ( $<\mathrm{scp}>1</$ Scp $>$-lactic Acid). Polym. Cryst. 2019, 2 (4). https://doi.org/10.1002/pcr2.10073.

(40) Damman, P.; Coppée, S.; Geskin, V. M.; Lazzaroni, R. What Is the Mechanism of Oriented Crystal Growth on Rubbed Polymer Substrates? Topography vs Epitaxy. J. Am. Chem. Soc. 2002, 124 (51), 15166-15167. https://doi.org/10.1021/ja0271451.

(41) Lin, C. W.; Ding, S. Y.; Hwang, Y. W. Interfacial Crystallization of Isotactic Polypropylene Molded against the Copper Surface with Various Surface Roughnesses Prepared by an Electrochemical Process. J. Mater. Sci. 2001, 36 (20), 4943-4948. https://doi.org/10.1023/A:1011848623699.

(42) Page, A. J.; Sear, R. P. Crystallization Controlled by the Geometry of a Surface. J. Am. Chem. Soc. 2009, 131 (48), 17550-17551. https://doi.org/10.1021/ja9085512.

(43) Di Profio, G.; Fontananova, E.; Curcio, E.; Drioli, E. From Tailored Supports to Controlled Nucleation: Exploring Material Chemistry, Surface Nanostructure, and Wetting Regime Effects in Heterogeneous Nucleation of Organic Molecules. Cryst. Growth Des. 2012, 12 (7), 3749-3757. https://doi.org/10.1021/cg3005568.

(44) Holbrough, J. L.; Campbell, J. M.; Meldrum, F. C.; Christenson, H. K. Topographical Control of Crystal Nucleation. Cryst. Growth Des. 2012, 12 (2), 750-755. https://doi.org/10.1021/cg201084j.

(45) Wang, B.; Wen, T.; Zhang, X.; Tercjak, A.; Dong, X.; Müller, A. J.; Wang, D.; Cavallo, D. Nucleation of Poly(Lactide) on the Surface of Different Fibers. Macromolecules 2019, 52 (16), 6274-6284. https://doi.org/10.1021/acs.macromol.9b01078.

(46) Lohmeijer, P. J. A.; Goossens, J. G. P.; Peters, G. W. M. Quiescent Crystallization of Poly(Lactic Acid) Studied by Optical Microscopy and Light-Scattering Techniques. J. Appl. Polym. Sci. 2017, 134 (10). https://doi.org/10.1002/app.44566.

(47) Boistelle, R.; Astier, J. P. Crystallization Mechanisms in Solution. J. Cryst. Growth 1988, 90 (1-3), 14-30. https://doi.org/10.1016/0022-0248(88)90294-1.

(48) Petrovsky, V. A.; Rakin, V. I.; Ruzov, V. P. Holographic Studies of the Solution Surrounding a Growing or Dissolving Crystal. J. Cryst. Growth 1982, 56 (1), 7-14. https://doi.org/10.1016/0022-0248(82)90006-9.

(49) Garside, J. Industrial Crystallization from Solution. Chemical Engineering Science. Pergamon January 1, 1985, pp 3-26. https://doi.org/10.1016/0009-2509(85)85043-0.

(50) Leoné, N.; Roy, M.; Saidi, S.; De Kort, G.; Hermida-Merino, D.; Wilsens, C. H. R. M. C. H. R. M. Improving Processing, Crystallization, and Performance of Poly- 1 -Lactide with an AmideBased Organic Compound as Both Plasticizer and Nucleating Agent. ACS Omega 2019, 4 (6), 10376-10387. https://doi.org/10.1021/acsomega.9b00848. 
(51) Wilsens, C. H. R. M.; Hawke, L. G. D.; De Kort, G. W.; Saidi, S.; Roy, M.; Leoné, N.;

Hermida-Merino, D.; Peters, G. W. M.; Rastogi, S. Effect of Thermal History and Shear on the Viscoelastic Response of IPP Containing an Oxalamide-Based Organic Compound.

Macromolecules 2019, 52 (7), 2789-2802. https://doi.org/10.1021/acs.macromol.8b02612. 



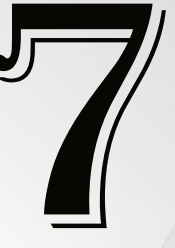

\section{EPILOGUE}





\subsection{Conclusions}

This thesis demonstrates that the synthesis of renewable pyrrolidone based dicarboxylic acids can be achieved via aza-Michael cascade cyclization, using itaconic acids and diamines/naturally occurring amino acids ${ }^{1}$. In addition, as described in Chapters 2 and 4, the synthesis procedures have been optimized to adhere to several principles of green chemistry; the reactions are performed in absence of solvent, water is employed as catalyst, and the monomers are obtained after purification via (re-)crystallization from water or other solvents. The developed pyrrolidone based dicarboxylic acids could successfully be polymerized into both thermoplastic and thermosets, making use of the thermal ring-opening addition reaction of bis(2-oxazolines $)^{2}$. In this case, a melt polymerization route is adopted to circumvent the use of solvents and to make their processing and production economically more attractive. One essential requirement for thermally curable resins, for adhesive purposes, is control over their curing kinetics. The newly developed pyrrolidone based dicarboxylic acids exhibit roughly a 100 fold higher reactivity towards ring-opening addition reactions with bis(2-oxazolines) compared to commonly used monomers such as sebacic acid ${ }^{1}$. Retrospectively, their use aids in reduction of curing time of these bis(2-oxazolines) based resins (Chapter 2). Though the developed materials can have glass transition temperatures $\left(T_{\mathrm{g}}\right)$ above $100{ }^{\circ} \mathrm{C}$, they are found to be plasticized with the absorption of water in ambient conditions, effectively restricting their use in humid conditions. Though this limits their potential use as an engineering plastic, it does exhibit a brittle-to-ductile transition, yielding tough thermosetting materials (Chapter 3). The adhesion strength of the developed materials on aluminum and polylactide at ambient conditions and after immersion in water is found to be comparable with the commercially available Gorilla glue ${ }^{3}$.

Preliminary tests indicate that the developed materials can be depolymerized rapidly in the presence of protease enzyme from Bacillus Sp. Furthermore, though slow hydrolysis proceeds over time, the presence of absorbed water does significantly hamper the chemical integrity of the material over a period of weeks to months. This makes these materials potential candidates for use in outdoor applications, for an example in agricultural seed coatings ${ }^{4-7}$, where moisture absorption together with its enzymatic degradation are $\operatorname{crucial}^{8}$ (Chapter 2). The materials synthesized in this thesis hold potential usage as medical adhesives or wound dressings ${ }^{9-11}$. For both applications, control over material stiffness and interaction with water is desired.

Furthermore, chemical recycling is found to be feasible with retrieval of the pyrrolidone monomers on exposing the thermosets to extreme hydrolytic conditions for sufficiently long time. The mechanical performance of the porous thermoset (obtained by partial hydrolysis) is evaluated (Chapter 3) and investigated for its 
potential in biomedical applications where the networks with different stiffness for cell growth are desired. Preliminary results suggest that these renewable thermosets are non-toxic towards cell growth. However, further pursuit of this topic is beyond the scope of this thesis and is recommended as future work.

The developed synthetic route allows the synthesis of pyrrolidone dicarboxylic acids derived from naturally occurring L-amino acids, retrospectively providing additional functionalities such as aromatic alcohol groups especially when using $L$-tyrosine. The synthesis and purification of these monomers is found to be challenging especially in their isolation in high purity and moderate yields $(>50 \%$, Chapter 4). The thermal ring-opening polymerization of bis(2-oxazolines) is reported to be effective in synthesizing (co) polymers of ( $L$-Tyr-PD and $L$-ph ala-PD) having pendant phenol groups without requiring any protective groups or solvents. By evaluating the viscoelastic response of the copolymers, it is demonstrated that the stress-relaxation response of the synthesized polymers can be tailored with the introduction of pendant groups, either by increasing the hydrogen bonding density (or secondary interactions in general) that increases the storage modulus of the synthesized amorphous polymer. Hence, these functional pyrrolidone monomers can be interesting in creating dynamic networks.

Furthermore, in addition to the recovery of the pyrrolidone monomers' the presence of other organic components, originating from the ring opened structure of the used 2-oxazolines, cannot be avoided even after purification and recrystallization with methanol. The potential of these compounds as additives for polylactide is evaluated and reported in Chapter 5. It is worthwhile to note that though the use of 2,5-furandicarboxylic acid derived bis(2-oxazolines) is desired from both kinetic and renewability perspectives (Chapter 2), its hydrolysis product N,N'-bis(2hydroxyethyl)furan-2,5-dicarboxamide (BHEF) is difficult to isolate and proves to hold no value when introduced into PLA as an additive. In this respect, the use of a terephthalic acid based bis(2-oxazoline) might be more preferred; its hydrolysis product N,N'-bis(2-hydroxyethyl)terephthalamide (BHET) can be easily isolated by recrystallization from water and acts both as a plasticizer and nucleating agent for PLA depending on the concentration of the additives used. To summarize the overall findings demonstrated in this thesis, a schematic overview is shown in scheme 7.1. 


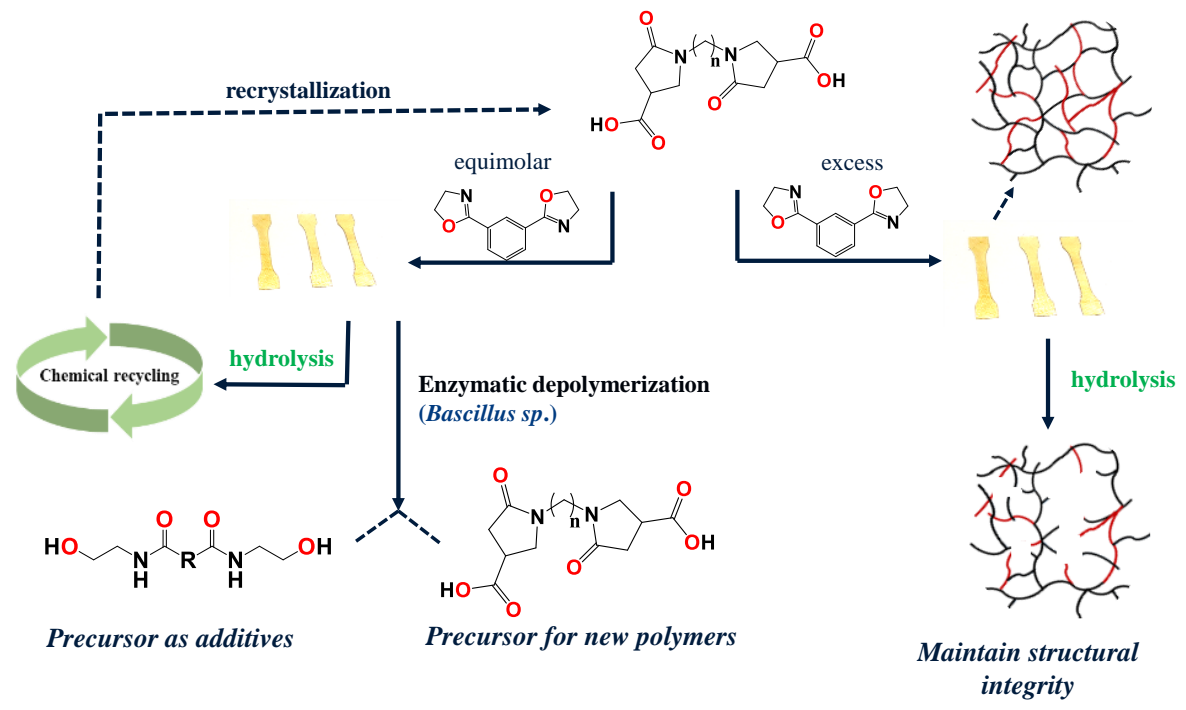

Scheme 7.1. A schematic overview showing potential of using renewable pyrrolidone based thermoplastics and thermosets.

While working with BHET as nucleating agents for PLA, it is observed that the cooling rate plays a significant role in enhancing nucleation efficiency of the matrix (PLA). Hence a comparative study is performed with a series of renewable oxalamide based organic compounds (OBOC) to evaluate their nucleation mechanism (Chapter 6). Interestingly, it is observed that the nucleation efficiency of the OBOCs increases at high cooling rates, making them more attractive for industrial processing conditions. In this case, the observed nucleation mechanism overrides the possibility of both epitaxy and soft epitaxy matching, while making both the surface roughness and local stress imposed on PLA as the responsible factors for its nucleation efficiency.

\subsection{Technological assessment}

The technological aspect of this thesis is foreseen in the development of biobased resins meeting the requirements of adhesives while using renewable pyrrolidone dicarboxylic acids (as monomers).

\subsubsection{Adhesives in coatings and bio-medical application}

The adhesives and sealants market are estimated to be 60 billion dollars by 2020, which is projected to reach 73.8 billion by $2024^{12}$. As discussed earlier we have confirmed literature observations that 2-oxazoline based resins exhibit excellent adhesion to glass, metals and other polar surfaces having amide or ester bonds ${ }^{13-16}$. 
Preliminary results confirm that the adhesion strength of the developed polymers on aluminium and polylactide (PLA) are comparable to that of commercially available Gorilla glue $^{3}$ as shown in figure 7.1. The developed monomers provide a toolbox for the development of adhesives with tailored chemical, physical and mechanical properties, providing potential to override the performance of commercially available non-renewable adhesives (as for example - epoxy resin).

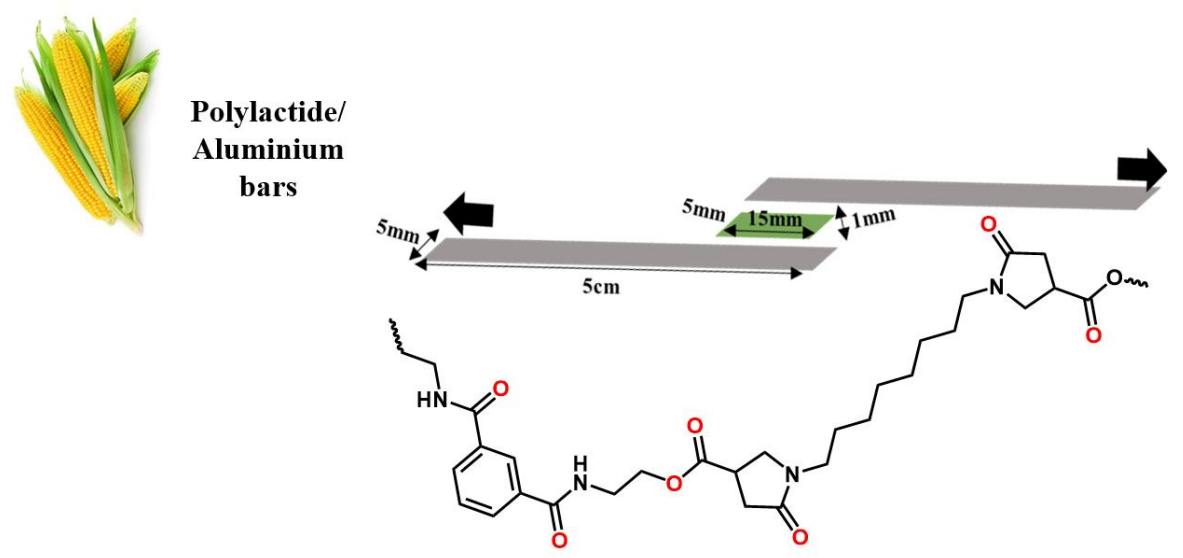

Figure 7.1. Schematic diagram of lap-shear tests performed to show the adhesion strength of the renewable pyrrolidone based resins.

Furthermore, these renewable thermosets, obtained in pure or partially hydrolyzed form, might hold potential in biomedical applications. Preliminary results suggest that these renewable thermosets are non-toxic towards cell growth.

\subsubsection{Towards enzymatic depolymerizable and regenerated resins:} Transition from traditional to sustainable (biobased) resins

Some of the traditional resins used like phenol formaldehyde ${ }^{17,18}$, epoxy resins ${ }^{19}$ etc. are either toxic or, non-recyclable, thereby ending as non-recyclable waste to landfill or aquatic contaminants. Among the adopted recycling methods, mechanical recycling is often used to reshape or reuse the polymers. While doing so, the mechanical performance of the recycled polymer deteriorates mainly due to chains scission. Similarly, biodegradation, or biological degradation via microbial actions, has been the goal for polymer degradation. However, this process does not allow us to recover the starting monomers (building blocks). In this respect, Enzymatic depolymerization can be an alternative approach, which will cause chain scission of the polymeric chains into oligomers or even into smaller repeating units (dimers or, even monomers) and can be recovered and reused for future 
applications $^{20-22}$. In addition to this, chemical recycling has been an emerging recycling possibility, where the starting building blocks (monomers) are recovered via a chemical technique like crystallization, precipitation, solvent extraction etc ${ }^{23}$. Sometime, the recovery of the pristine starting materials (monomers) is not possible, however the recovered organic compounds are used as a new building block (as monomer or oligomers) for further usage ${ }^{24}$. The steps involved in chemical recycling involves also pyrolysis, thermal recycling and solvolysis ${ }^{25,26}$.

The polymers developed via ring opening polymerization of bis(2oxazolines) with renewable pyrrolidone dicarboxylic acids are of special interest when it comes to chemical recycling due to the presence of numerous hydrolysable ester and amide bonds ${ }^{2}$. These amorphous polymers with ease in moisture absorption enables both enzymatic depolymerization ${ }^{1}$ and chemical recycling as recycling options (Chapter $2 \& 3$ ). These recycling steps allow recovery of renewable starting pyrrolidone monomers with a possibility of higher yield and purity, thus providing an excellent basis for chemical polymer recycling. Both these recycling steps make the synthesized amorphous polymers attractive to the adhesives and coating industries, where the traditional thermosets are known for their non-degradability and non-renewable toxic nature ${ }^{18}$. These sustainable approaches are interesting findings, while aiming towards achieving circularity of these (biobased) polymers ${ }^{27,28}$. However, the efficiency of their chemical recycling, i.e. recovery of the starting pyrrolidone monomers and evaluation of the performance of regenerated renewable bis(2-oxazolines) based resins is not evaluated earlier. Hence, a case study is conducted to comment on the chemical recycling efficiency of these renewable 2oxazoline based polymers. In this study, recovery of the $\mathrm{C}_{10}$ spacer pyrrolidone monomer is chosen, due to its higher crystallization efficiency owing to its larger spacer length $\left(\mathrm{BP}-\mathrm{C}_{10}\right.$, Chapter 2). The recycling efficiency of the pyrrolidone monomers, results into moderate to higher yield and purity (table $7.1 \&$ figure 7.2).

Table 7.1: Recovery efficiency (\%) of the used pyrrolidone monomer (BP $\left.C_{10}\right)$ obtained after the chemical recycling of the renewable 2-oxazoline based polymers $B P C_{10}$ and 1,3-IAox.

\begin{tabular}{|ccc|}
\hline Recycling of $\mathrm{BPC}_{10}$ & Purity (\%) & Yield (\%) \\
\hline $1^{\text {st }}$ recycling step & $>90$ & 50 \\
\hline $2^{\text {nd }}$ recycling step & $>90$ & 63 \\
\hline $3^{\text {rd }}$ recycling step & $>90$ & 67 \\
\hline
\end{tabular}




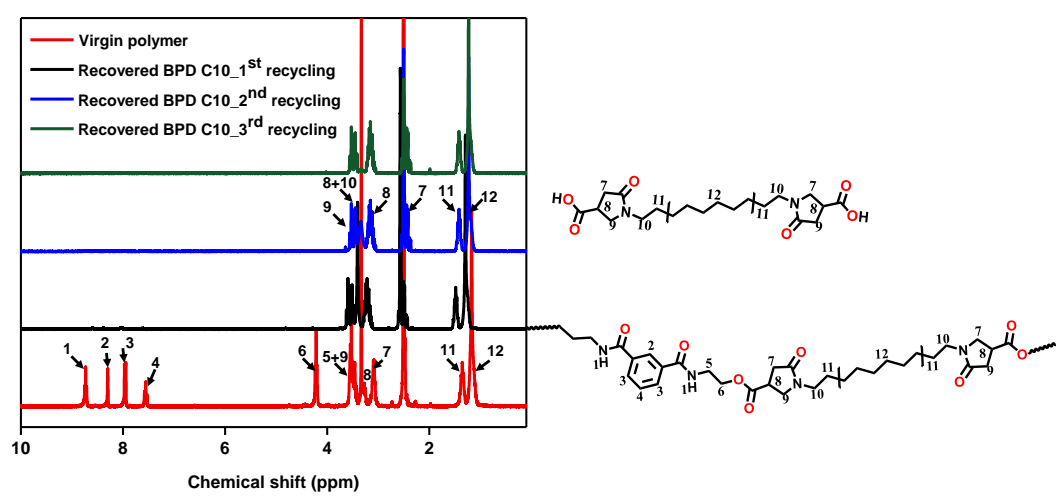

Figure 7.2. ${ }^{1} \mathrm{H}$-NMR spectra of, from bottom to top, polymer $B P C_{10}$ and 1,3-IAox directly after synthesis (red), recovered $B P C_{10}$ monomer after $1^{\text {st }}$ recycling step (black), $2^{\text {nd }}$ recycling step (blue) and $3^{\text {rd }}$ recycling step (green).

Other pyrrolidone based dicarboxylic acids with even aliphatic spacers are evaluated for this recycling efficiency tests. However it was observed that not all even spaced pyrrolidone monomers show the same recycling efficiency, likely resulting from their different crystallization rates $^{1}$ as evident from table 7.2 and elaborated in Chapter 2.

Table 7.2: Recovery efficiency (\%) of the various aliphatic even spacer pyrrolidone monomers obtained after the chemical recycling of the renewable 2-oxazoline based polymers.

\begin{tabular}{|cc|}
\hline Polymer & Recovery of monomers, yield (\%) \\
\hline BPC $_{2}$ & 85 \\
\hline BPC $_{4}$ & Not isolated \\
\hline BPC $_{6}$ & 13 \\
\hline BPC $_{8}$ & 53 \\
\hline BPC $_{10}$ & 67 \\
\hline BPC $_{12}$ & 63 \\
\hline
\end{tabular}

The recovered pyrrolidone monomers are used in polymerization with (bis)2oxazolines. The synthesized polymer demonstrates that the regenerated (bis)2- 
oxazoline based polymers have comparable mechanical performances, even after three recycling steps (table 7.3 and figure 7.3). Some traces of branching (oligomers), observed from the GPC data, cannot be excluded. However, no traces of impurity are observed in the ${ }^{1} \mathrm{H}$ NMR of the recovered $\mathrm{BPC}_{10}$ monomer, which might be limitation of the employed ${ }^{1} \mathrm{H}$ NMR instrument.

However, the differences in glass transition temperature $\left(T_{g}\right)$ of the regenerated polymers may be attributed to the presence of branching, as reflected from their poly dispersity index, which inhibits its chain mobility thereby increasing the glass transition temperature significantly.
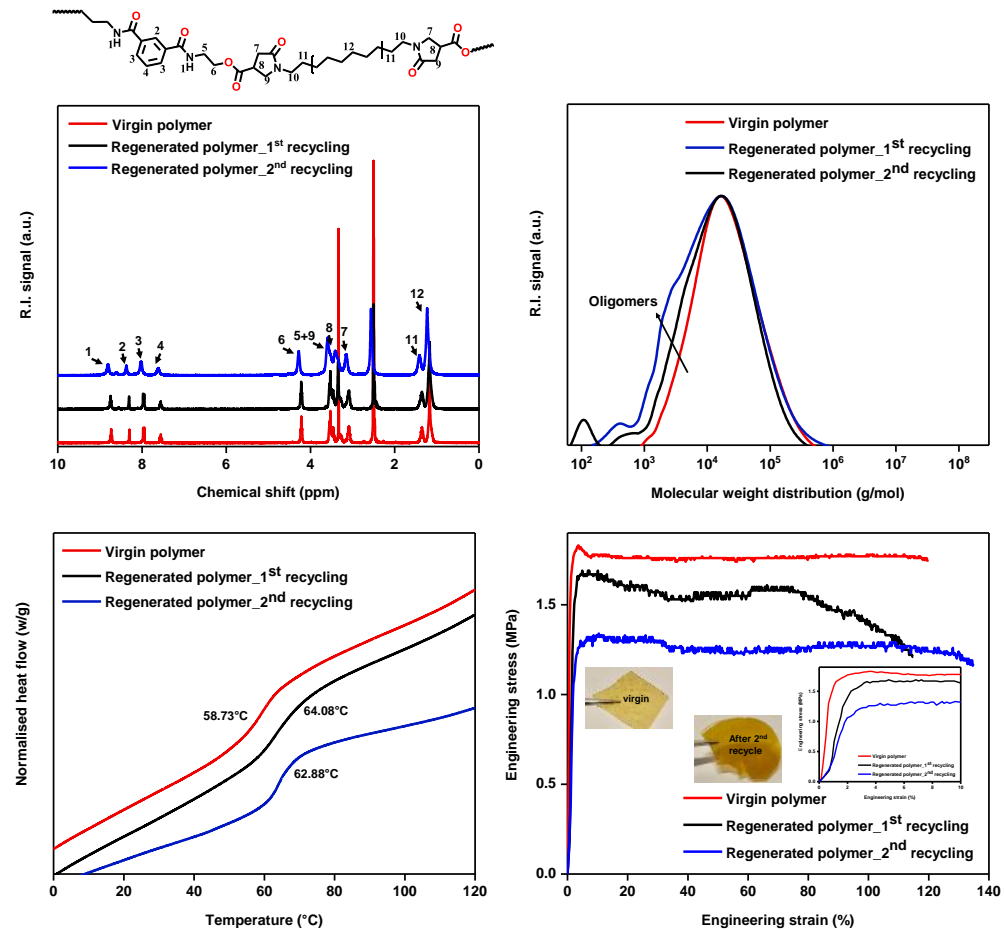

Figure 7.3. (Top, left $)^{l} H$-NMR spectra of, from bottom to top, polymer directly after synthesis (red), regenerated polymer obtained after $1^{\text {st }}$ recycling step (black), $2^{\text {nd }}$ recycling step (blue). (Top, right) GPC traces of polymer directly after synthesis (red), regenerated polymer after $1^{\text {st }}$ recycling step (black), $2^{\text {nd }}$ recycling step (blue) obtained after curing for 60 minutes at $180{ }^{\circ} \mathrm{C}$. (Bottom, left) Differential scanning calorimetry (DSC) traces of the as synthesized polymer (red), regenerated polymer obtained after 1 st recycling (black) and $2^{\text {nd }}$ recycling (blue) after curing at $180{ }^{\circ} \mathrm{C}$ for one hour. (Bottom, right) Characteristic engineering stress vs engineering strain curves for virgin polymer (red), $1^{\text {st }}$ regenerated polymer (black) and $2^{\text {nd }}$ regenerated 
polymer (blue) after exposed to air for one day. Note, the inset displays the same traces, only at low engineering strain.

Table 7.3. Molecular weight distribution of the virgin polymer containing recovered $B P C_{10}$ and 1,3-IAox (red), regenerated polymer after $1^{\text {st }}$ recycling (blue) and $2^{\text {nd }}$ recycling (black).

\begin{tabular}{|ccc|}
\hline Polymer & $\mathrm{M}_{\mathrm{w}}$ & PDI \\
\hline Virgin polymer & 47,800 & 4.99 \\
\hline Regenerated polymer_1 ${ }^{\text {st }}$ recycling & $\mathbf{5 4 , 5 0 0}$ & 15.6 \\
\hline Regenerated polymer_2 $2^{\text {nd }}$ recycling & 39,400 & 21.6 \\
\hline
\end{tabular}

Similar investigations are performed with semi-crystalline polymers (obtained by using a combination of sebacic acid with 1,3-IAox and 1,4-IAox bis(oxazolines)) which as anticipated, showed chemical recycling is ineffective, Thus, a proper choice of monomers is critical while designing a degradable or recyclable polymer.

\subsubsection{Developing reprocessable (dynamic) networks in renewable (biobased) resins: A more sustainable future}

The synthesized functional pyrrolidone dicarboxylic acids are interesting in developing functional (biobased) thermoplastics, which can be further tailored via pre/post-modification to introduce dynamic networks via ionic interaction or hydrogen bonding or by modifications of pendant groups. Due to the presence of electron rich polymer backbone, any Lewis acids or metal ions can form co-ordinate bond via ionic interactions ${ }^{29,30}$ thereby forming ionic networks. Furthermore, the presence of numerous esters, amides and pendant functionalities like phenolic hydroxyl group, phenyl rings enhance the hydrogen bonding density, thereby making these renewable thermoplastics equivalent to thermosets. These secondary interactions (ionic or hydrogen bonding) introduces dynamic networks via clusters formation, below its dissociation temperature, thereby increasing its storage modulus with enhanced stress relaxation behavior above the glass transition temperature. The dissociation of these clusters lowers the viscosity of these amorphous polymer, thereby enable reprocessing or reshaping of these thermosets like thermoplastic upon their damage or failure, which is considered as a most sustainable mechanistic perspective ${ }^{31}$. This approach enables development of mechanically recyclable dynamic materials. The most interesting aspect of these secondary interactions lies in the fact that their dissociation and/or association kinetics is temperature dependent. 
However, this dissociation-association kinetics is solely dependent on the nature of the crosslinking in polymer matrix invoking dynamics at the local length scales. If a targeted research is conducted, keeping commercialization in mind, these synthesized functional pyrrolidone monomers hold commercial potential for adhesives.
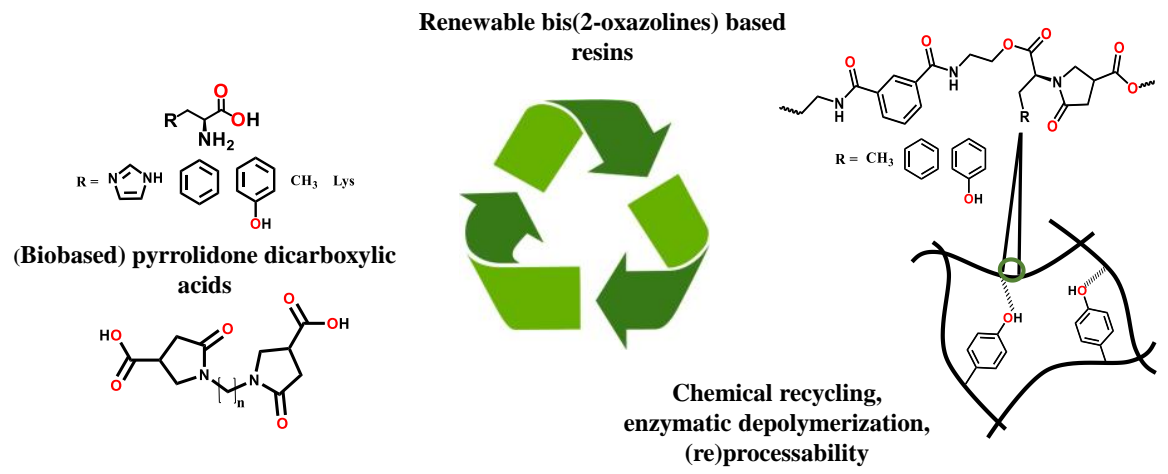

Scheme 7.2. A representation showing circularity of the synthesized biobased pyrrolidone monomers and the derived renewable 2-oxazoline based polymers showing secondary interactions.

Combining the possibility of monomers acquisition from renewable sources, and recycling possibility of the synthesized polymers from these monomers, the proposed synthesis and recycling route in the thesis holds potential in the development of a new class of resins that are likely to be commercially attractive (Scheme 7.2). Overall, these findings are motivational towards conducting further research in developing renewable monomers as precursors for biobased polymers (thermoplastics and thermosets) while utilizing only renewable resources. All these factors govern the importance of using these pyrrolidone monomers (both aliphatic ones derived from diamines, and functional ones derived from $L$-amino acids) in developing a sustainable future.

As discussed earlier, the potential application of these developed renewable (biobased) resins include adhesives for renewable (bio)composites, where enzymatic depolymerization and (both mechanical and chemical) recycling are desired.

\subsection{Suggestions and outlook}

As explained earlier, the target of using these synthesized pyrrolidone dicarboxylic acids involve green synthesis routes and biobased sources that can be obtained in higher yield and purity. This thesis demonstrates and confirms the potential of these pyrrolidone dicarboxylic acids as an alternative biobased monomer towards developing biobased thermoplastics and thermosets. Nevertheless, during the 
execution of this study several questions were identified, which will further advance the understanding in the field. Some are mentioned herewith in the form of recommendation for future work.

- Using the knowledge gained in this work, one may conclude that the developed materials will not be desired as engineering plastics. However, given their potential for recyclability, it is recommended to focus on the development of new resins that can be chemically recycled (fully) after their usage.

- Second possibility is to evaluate the potential of these materials in composites, as the matrix can be ductile. (Though water absorption will induce a lot of stress on the matrix/filler surface and might not work as desired).

- The developed renewable resins and porous thermosets obtained after partial hydrolysis, might be interesting for bio-medical applications (Chapter 3). The control over stiffness as a function of moisture exposure time makes it interesting to mimic the living tissue with varying stiffness. Preliminary results suggest that these renewable (bis)2-oxazoline based thermosets are non-toxic towards cell growth. Hence, further research will shed light on this goal, which can bring a new perspective and motivation towards using these (bis)2-oxazolines in biomedical field as adhesives.

- The synthesized new functional pyrrolidone monomers (Chapter 4) derived from $L$-amino acids, opens a new opportunity to introduce dynamic networks via ionic bonding, hydrogen bonding with reprocessing efficiency without substantial loss of their mechanical performance. This approach would bring new in-sight for defining future research goals. 


\subsection{References}

(1) Roy, M.; Noordzij, G. J.; Van Den Boomen, Y.; Rastogi, S.; Wilsens, C. H. R. M. Renewable (Bis)Pyrrolidone Based Monomers as Components for Thermally Curable and Enzymatically Depolymerizable 2-Oxazoline Thermoset Resins. ACS Sustain. Chem. Eng. 2018, 6 (4). https://doi.org/10.1021/acssuschemeng.7b04716.

(2) Roy, M.; Wilsens, C. H. R. M. C. H. R. M.; Leoné, N.; Rastogi, S. Use of Bis(Pyrrolidone)Based Dicarboxylic Acids in Poly(Ester-Amide)-Based Thermosets: Synthesis, Characterization, and Potential Route for Their Chemical Recycling. ACS Sustain. Chem. Eng. 2019, 7 (9), 8842-8852. https://doi.org/10.1021/acssuschemeng.9b00850.

(3) Jenkins, C. L.; Siebert, H. M.; Wilker, J. J. Integrating Mussel Chemistry into a Bio-Based Polymer to Create Degradable Adhesives. Macromolecules 2017, 50 (2), 561-568. https://doi.org/10.1021/acs.macromol.6b02213.

(4) Pramanik, S.; Konwarh, R.; Sagar, K.; Konwar, B. K.; Karak, N. Bio-Degradable Vegetable Oil Based Hyperbranched Poly(Ester Amide) as an Advanced Surface Coating Material. Prog. Org. Coatings 2013, 76 (4), 689-697. https://doi.org/10.1016/j.porgcoat.2012.12.011.

(5) Adhikari, R.; Bristow, K. L.; Casey, P. S.; Freischmidt, G.; Hornbuckle, J. W.; Adhikari, B. Preformed and Sprayable Polymeric Mulch Film to Improve Agricultural Water Use Efficiency. Agric. Water Manag. 2016, 169, 1-13. https://doi.org/10.1016/j.agwat.2016.02.006.

(6) Lahalih, S. M.; Akashah, S. A.; Al-Hajjar, F. H. Development of Degradable Slow Release Multinutritional Agricultural Mulch Film. Ind. Eng. Chem. Res. 1987, 26 (11), 2366-2372. https://doi.org/10.1021/ie00071a032.

(7) Lu, P.; Zhang, Y.; Jia, C.; Li, Y.; Mao, Z. Use of Polyurea from Urea for Coating of Urea Granules. Springerplus 2016, 5 (1). https://doi.org/10.1186/s40064-016-2120-x.

(8) Clendinning, R. A.; Potts, J. E.; Cornell, S. W. Environmantally Degradable-Biodegradable Blend of an Oxyalkanoyl Polymer and an Environmentally Degradable Ethylene Polymer. Patent No: 3,867,324, 1975.

(9) Winnacker, M.; Rieger, B. Poly(Ester Amide)s: Recent Insights into Synthesis, Stability and Biomedical Applications. Polym. Chem. 2016, 7 (46), 7039-7046. https://doi.org/10.1039/c6py01783e.

(10) Fonseca, A. C.; Gil, M. H.; Simões, P. N. Biodegradable Poly(Ester Amide)s - A Remarkable Opportunity for the Biomedical Area: Review on the Synthesis, Characterization and Applications. Prog. Polym. Sci. 2014, 39 (7), 1291-1311. https://doi.org/10.1016/j.progpolymsci.2013.11.007.

(11) Manolakis, I.; Noordover, B. A. J.; Vendamme, R.; Eevers, W. Novel L -DOPA-Derived Poly(Ester Amide)s: Monomers, Polymers, and the First L -DOPA-Functionalized Biobased Adhesive Tape. Macromol. Rapid Commun. 2014, 35 (1), 71-76.

https://doi.org/10.1002/marc.201300750. 
(12) Adhesives \& Sealants Market by Adhesive Formulating Technology (Water-Based, SolventBased, Hot-Melt, Reactive), Sealant Resin Type (Silicone, Polyurethane, Plastisol, Emulsion, Butyl, Polysulfide), Application, Region - Global Forecast to 2024

https://www.marketsandmarkets.com/Market-Reports/adhesive-sealants-market-421.html.

(13) Walton, C. D.; Grove, L. Creping Adhesives Containing Oxazoline Polymers and Methods of Use Thereof. Patent No: 5,980,690, 1999.

(14) Takeharu TAJIMA, S. (JP). Fiber Cross-Linked Body and Manufacturing Method of Fiber Cross-Linked Body. Patent No: US 2010/0215957 A1, 2010.

(15) Fairchok, W. J.; P., T. B.; Friedrich, R. E.; McKinley, M. J. Transmitted Poly-2-Oxazoline Composition Useful as Wetting Agent for Polymer and Absorbents for Polar Materials. Patent No; 4,582,877, 1986.

(16) Erukhimovich, I.; de la Cruz, M. O. Chemical Modification of Polyamide-6 by Chain Extension with 2,20-Bis(2-Oxazoline). J. Polym. Sci. Part B Polym. Phys. 2006, 45 (October), 1976-1982. https://doi.org/10.1002/polb.

(17) Gürbilek, N. Summary for Policymakers. In Climate Change 2013 - The Physical Science Basis; Intergovernmental Panel on Climate Change, Ed.; Cambridge University Press: Cambridge, 2013; Vol. 53, pp 1-30. https://doi.org/10.1017/CBO9781107415324.004.

(18) Anderson, R. C.; Stock, M. F.; Sawin, R.; Alarie, Y. Toxicity of Thermal Decomposition Products of Urea Formaldehyde and Phenol Formaldehyde Foams. Toxicol. Appl. Pharmacol. 1979, 51 (1), 9-17. https://doi.org/10.1016/0041-008X(79)90003-6.

(19) BOURNE, L. B.; MILNER, F. J.; ALBERMAN, K. B. Health Problems of Epoxy Resins and Amine-Curing Agents. Br. J. Ind. Med. 1959, 16 (2), 81-97. https://doi.org/10.1136/oem.16.2.81.

(20) Rollings, J. Enzymatic Depolymerization of Polysaccharides. Carbohydr. Polym. 1985, 5 (1), 37-82. https://doi.org/10.1016/0144-8617(85)90018-9.

(21) Li, B.; Wang, Y.; Mahmood, N.; Yuan, Z.; Schmidt, J.; Xu, C. (Charles). Preparation of BioBased Phenol Formaldehyde Foams Using Depolymerized Hydrolysis Lignin. Ind. Crops Prod. 2017, 97, 409-416. https://doi.org/10.1016/j.indcrop.2016.12.063.

(22) Concha Olmos, J.; Zúñiga Hansen, M. E. Enzymatic Depolymerization of Sugar Beet Pulp: Production and Characterization of Pectin and Pectic-Oligosaccharides as a Potential Source for Functional Carbohydrates. Chem. Eng. J. 2012, 192, 29-36. https://doi.org/10.1016/j.cej.2012.03.085.

(23) Matsumura, S. Enzyme-Catalyzed Synthesis and Chemical Recycling of Polyesters. Macromol. Biosci. 2002, 2 (3), 105. https://doi.org/10.1002/1616-5195(20020401)2:3<105::AIDMABI105>3.0.CO;2-K.

(24) Lin, C. H.; Lin, H. Y.; Liao, W. Z.; Dai, S. A. Novel Chemical Recycling of Polycarbonate (PC) Waste into Bis-Hydroxyalkyl Ethers of Bisphenol A for Use as PU Raw Materials. Green Chem. 2007, 9 (1), 38-43. https://doi.org/10.1039/b609638g. 
(25) Cadena, L. E. S.; Arroyo, Z. G.; Lara, M. A. G.; Quiroz, Q. D. Cell-Phone Recycling by Solvolysis for Recovery of Metals. J. Mater. Sci. Chem. Eng. 2015, 03 (01), 52-57. https://doi.org/10.4236/msce.2015.31008.

(26) Arturi, K. R.; Sokoli, H. U.; Søgaard, E. G.; Vogel, F.; Bjelić, S. Recovery of Value-Added Chemicals by Solvolysis of Unsaturated Polyester Resin. J. Clean. Prod. 2018, 170, 131-136. https://doi.org/10.1016/j.jclepro.2017.08.237.

(27) Karpudewan, M.; Ismail, Z.; Roth, W. M. Ensuring Sustainability of Tomorrow through Green Chemistry Integrated with Sustainable Development Concepts (SDCs). Chem. Educ. Res. Pract. 2012, 13 (2), 120-127. https://doi.org/10.1039/c1rp90066h.

(28) Prieto-Sandoval, V.; Jaca, C.; Ormazabal, M. Towards a Consensus on the Circular Economy. J. Clean. Prod. 2018, 179, 605-615. https://doi.org/10.1016/j.jclepro.2017.12.224.

(29) Bode, S.; Zedler, L.; Schacher, F. H.; Dietzek, B.; Schmitt, M.; Popp, J.; Hager, M. D.; Schubert, U. S. Self-Healing Polymer Coatings Based on Crosslinked Metallosupramolecular Copolymers. Adv. Mater. 2013, 25 (11), 1634-1638. https://doi.org/10.1002/adma.201203865.

(30) Xu, C.; Huang, X.; Li, C.; Chen, Y.; Lin, B.; Liang, X. Design of “ Zn 2+ Salt-Bondings” Cross-Linked Carboxylated Styrene Butadiene Rubber with Reprocessing and Recycling Ability via Rearrangements of Ionic Cross-Linkings. 2016, 2-11. https://doi.org/10.1021/acssuschemeng.6b01897.

(31) Winne, J. M.; Leibler, L.; Prez, F. E. Du. Polymer Chemistry a Mechanistic Perspective. 2019, 6091-6108. https://doi.org/10.1039/c9py01260e. 



\section{APPENDIX A}





\section{Supporting information for Chapter 2}

\section{2-oxazoline conversion determination procedure using NMR spectroscopy}

To study the reaction rate of polymerization, the 2-oxazoline conversion was monitored during the polymerization of an equimolar ratio of 2,5-FDCAox with $\mathrm{BP}_{-} \mathrm{C}_{8}$ and IAox, both with $\mathrm{BP}-\mathrm{C}_{8}$, at $180^{\circ} \mathrm{C}$. Samples were collected during curing, dissolved in deuterated DMF (FDCAox) or DMSO (IAox), and analyzed with ${ }^{1} \mathrm{H}$ NMR. Generally, the 2-oxazoline conversion was calculated from ${ }^{1} \mathrm{H}$ NMR spectra of the polymer obtained after polymerization for a defined reaction time. To this end, the conversion was calculated by dividing the value of the integral of the $\mathrm{CH}_{2} \mathrm{O}$ resonance of the ring-opened 2-oxazoline ring by the total number of $\mathrm{CH}_{2} \mathrm{O}$ protons present in the system. The total amount of 2-oxazoline was determined summing up the integrals of the $\mathrm{CH}_{2} \mathrm{O}$ resonance of the ring-opened 2-oxazoline ring (Signals A in Figure A1 and A2) and the integral of the $\mathrm{CH}_{2} \mathrm{O}$ resonance of the non-reacted 2-oxazoline ring (Signals B in Figure A1 and A2). Figures A1 and A2 depict the exemplary NMR spectra in the region of interest $(3-5 \mathrm{ppm})$ taken during polymerization of FDCAox and IAox based systems, respectively.

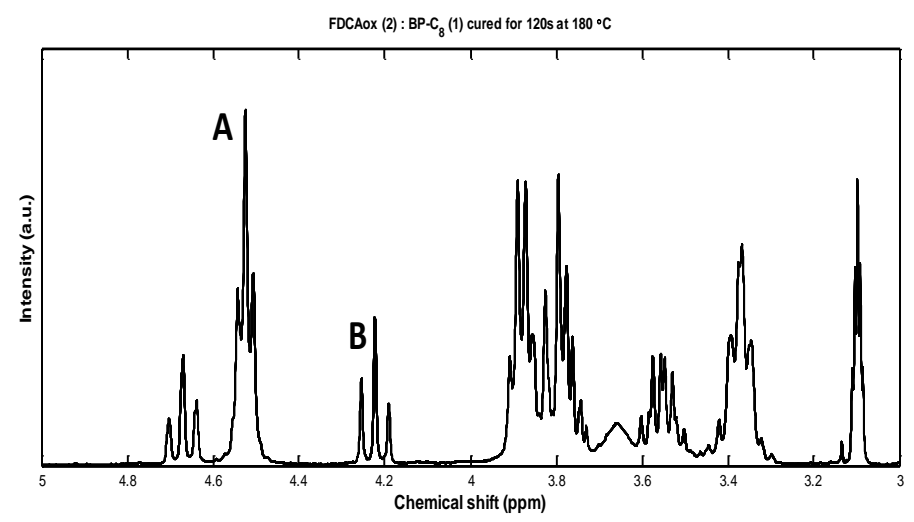

Figure A1. ${ }^{1} \mathrm{H} N \mathrm{NR}$ spectrum in the range of $4-8 \mathrm{ppm}$ of the polymerization product of FDCAox with BP-C $C_{8}$ after 120 seconds of curing (d-DMF as solvent). A and $\mathrm{B}$ highlight the $\mathrm{CH}_{2} \mathrm{O}$ resonance of the ring-opened 2-oxazoline ring and the unreacted 2-oxazoline ring, respectively. 


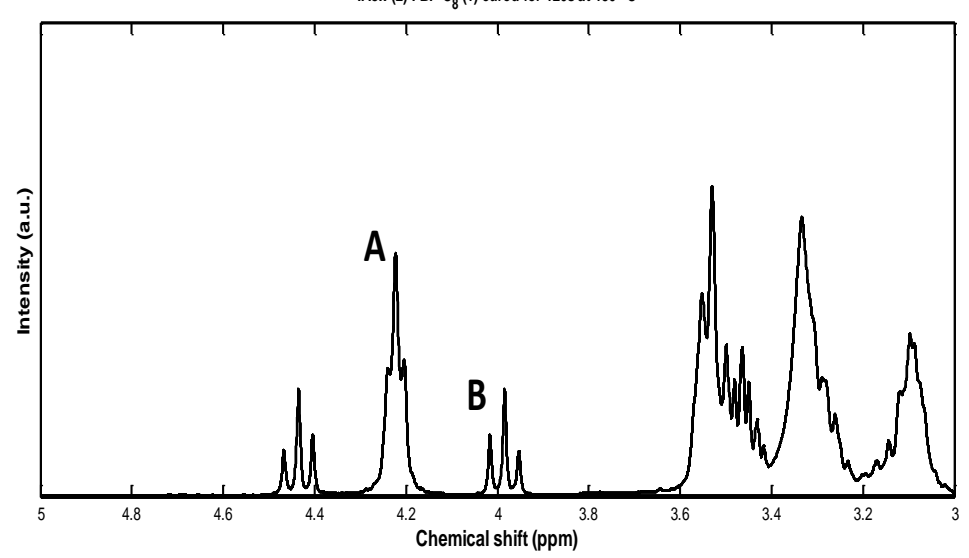

Figure A2. ${ }^{1} \mathrm{H}$ NMR spectrum in the range of $3-5$ ppm of the polymerization product of IAox with BP-C $C_{8}$ after 120 seconds of curing (d-DMSO as solvent). A and $\mathrm{B}$ highlight the $\mathrm{CH}_{2} \mathrm{O}$ resonance of the ring-opened 2-oxazoline ring and the unreacted 2-oxazoline ring, respectively.

\section{Relation between glass transition temperature of BP-Cx monomers and the hydrogen bonding density}

The hydrogen bonding density of the $\mathrm{BP}-\mathrm{C}_{\mathrm{x}}$ monomers is defined as the number of hydrogen bonds formed by one $\mathrm{BP}-\mathrm{C}_{\mathrm{x}}$ molecule divided over the number of atoms present in between the two carboxylic acid moieties (highlighted in red for BP- $\mathrm{C}_{2}$ in Figure A3). When plotting the hydrogen bonding density of the various ${ }_{B P}-C_{x}$ monomers as a function of their glass transition temperature (Figure A4), one can see that the glass transition temperature is linearly related to the hydrogen bonding density.

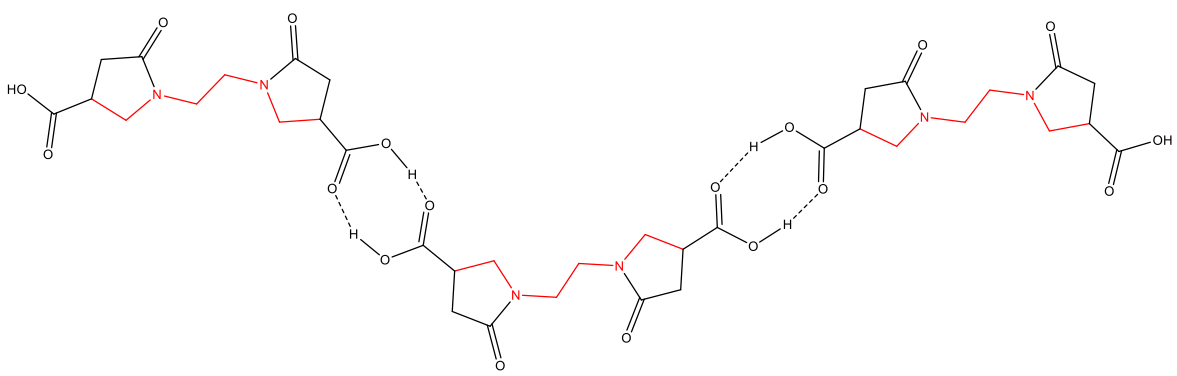

Figure A3. Schematic representation of the hydrogen bonding occurring in $B P-C_{2}$. 


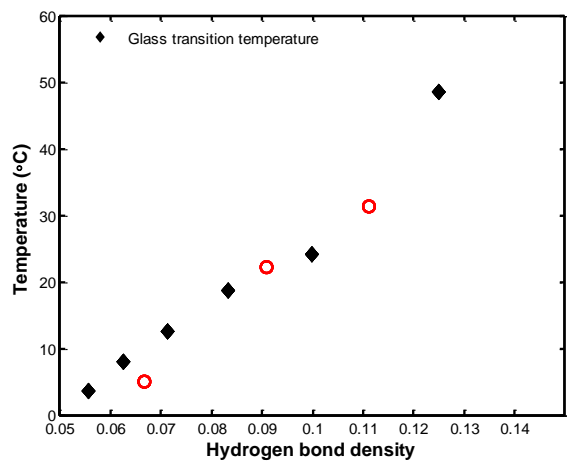

Figure A4. Glass transition temperature as a function of hydrogen bonding density.

\section{FTIR analysis of IAox and/or TPP based systems}

Figure A5 depicts the FTIR traces of IAox : Sebacic acid (A) and IAox : $\mathrm{BP}_{-} \mathrm{C}_{8}(\mathrm{~B})$ resins over time, as observed during curing performed at $180{ }^{\circ} \mathrm{C}$. Similar to the description in the original manuscript, three distinct vibrational bands can be observed: I corresponds to the benzene ring vibration $\left(815 \mathrm{~cm}^{-1}\right)$, II corresponds to the oxazoline ring vibration $\left(911 \mathrm{~cm}^{-1}\right)$, and III corresponds to the tertiary amide bonds vibration $\left(1425 \mathrm{~cm}^{-1}\right)$. Again, I remain constant during the curing process and is therefore used as internal standard. Signal II decreases significantly over time, resulting from the ring-opening addition reaction of the 2-oxazoline ring with the carboxylic acid group. However, in contrast to the system containing FDCAox depicted in Figure 2.7 of the original manuscript, no significant changes in peak area of resonance III is observed, indicating that the $k_{2}$ reaction constant is significantly lower than for systems containing FDCAox. As can be seen from Figure A5., vibration II in spectra containing IAox is overlapping with other vibrations in the FTIR spectra, making accurate integration of the peak area challenging. Therefore, no data fitting to determine the $k_{1}$ and $k_{2}$ reaction constants was performed. Additionally, the FTIR traces used to generate the data in Figure $2.8 \mathrm{~B}$, for $\mathrm{BP}-\mathrm{C}_{8}$ systems containing $1 \mathrm{wt} \%$ TPP are provided in Figure A6. 

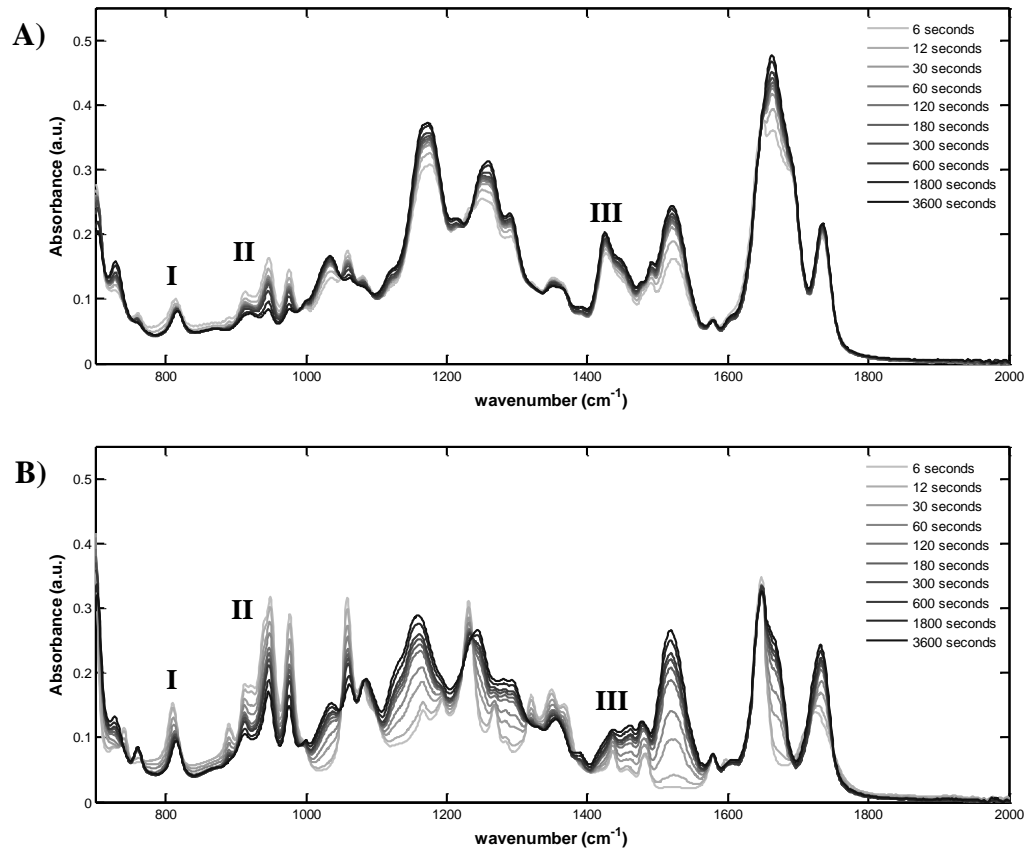

Figure A5. FTIR spectra (700-2000 $\left.\mathrm{cm}^{-1}\right)$ obtained during the on-line monitoring of the curing of (A) BP-C (1): IAox (2) and (B) Sebacic acid (1) : IAox (2) systems. Vibrational bands I, II, and III denote the characteristic vibrations from the benzene ring, 2-oxazoline ring, and tertiary amide bonds respectively. 

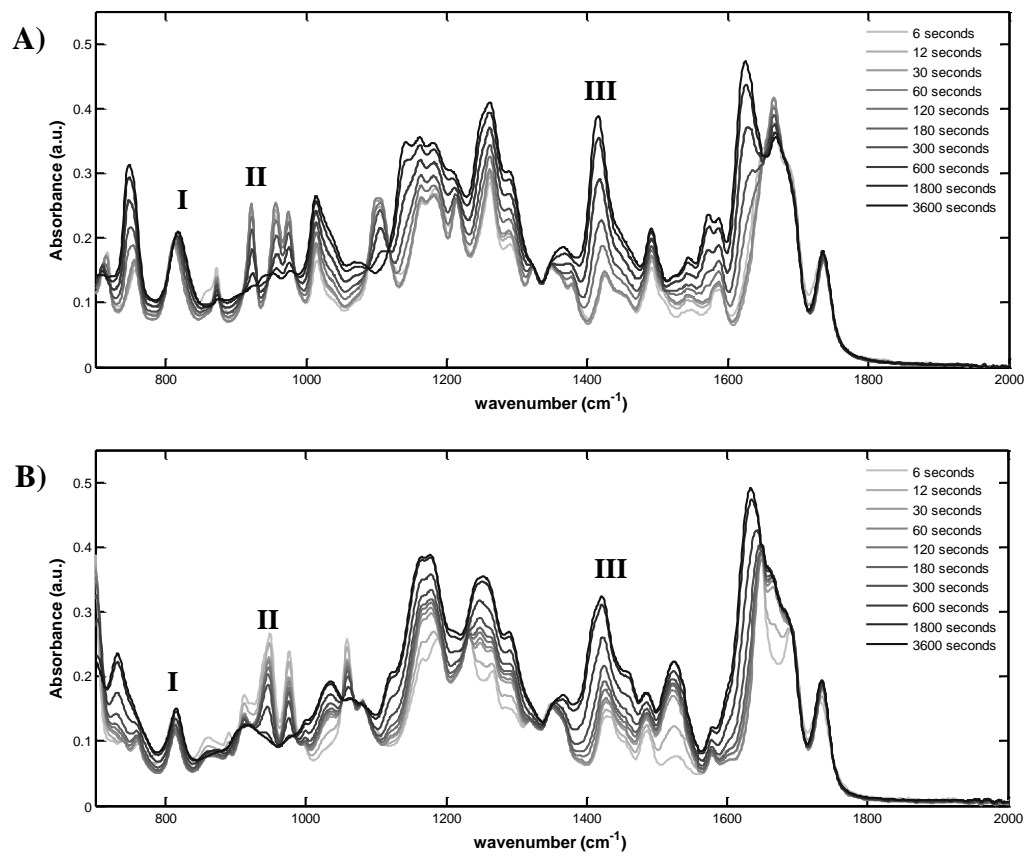

Figure A6. FTIR spectra (700-2000 $\left.\mathrm{cm}^{-1}\right)$ obtained during the on-line monitoring of the curing of (A) BP-C $C_{8}(1):$ FDCAox (2) and (B) BP-C $C_{8}(1):$ IAox (2) systems, both containing 1 wt\% TPP as catalyst. Vibrational bands I, II, and III denote the characteristic vibrations from the aromatic rings, 2-oxazoline ring, and tertiary amide bonds respectively.

\section{Verification of enhanced $k_{1}$ reaction constant on the rate of cross- linking}

From the findings in the original manuscript we observe that the $k_{1}$ reaction constant is significantly enhanced for systems containing $\mathrm{BP}-\mathrm{C}_{\mathrm{x}}$ monomers compared to systems containing sebacic acid. To verify is this also results in a more rapid cross-linking, we have predicted the average molecular weight increase over time taking into account the reaction kinetics described in equations $(\mathbf{2 . 1})-\mathbf{( 2 . 3 )}$ of the manuscript. The average molecular weight in the system can be approximated through subtraction of the reacted carboxylic acid and formed tertiary amide functionalities (A4) from the total number of monomers initially present in the system $\left(\right.$ Chains $_{0}$ in (A5)). Accordingly, the average molecular weight per chain $\left(\mathrm{Mw}_{\mathrm{t}}\right)$ in $\mathrm{g} / \mathrm{mol}$ is defined as the total weight of the system (1 kilogram used for the model) divided by the number of chains present in the systems at any given time $t$ (Chains ${ }_{\mathrm{t}}$ in (A6)). 
(A4) Chains $_{t}=$ Chains $_{0}-\left([\text { Acid }]_{0}-[\text { Acid }]_{t}\right)+\left([\operatorname{TertA}]_{t}-[\text { TertA }]_{0}\right)$

(A5) Chainso $=0.5 *[\mathrm{OX}]_{0}+0.5 *[\text { Acid }]_{0}$

(A6) $\mathrm{Mwt}=(\text { Chainst })^{-1} * 10^{3}$

Here $[\text { Acid }]_{0},[\mathrm{OX}]_{0}$, and $[\text { Tert } \mathrm{A}]_{0}$ are the carboxylic acid, 2-oxazoline, and tertiary amide concentrations at the start of the reaction. Similarly, [Acid] $]_{t}$ and $[\text { Tert } \mathrm{A}]_{\mathrm{t}}$ are the concentrations of carboxylic acid and tertiary amide functionalities at time $t$ during curing. Using this approach, the molecular weight increase during curing is predicted using the $k_{1}$ and $k_{2}$ parameters obtained from FTIR data fitting (Figure A7). These predictions confirm that the enhanced $k_{1}$ reaction constant increases both the molecular weight build-up in the early stage of polymerization and decreases the time-to-crosslink (defined as the time where the molecular weights surpass $10^{6} \mathrm{~g} / \mathrm{mol}$ ).
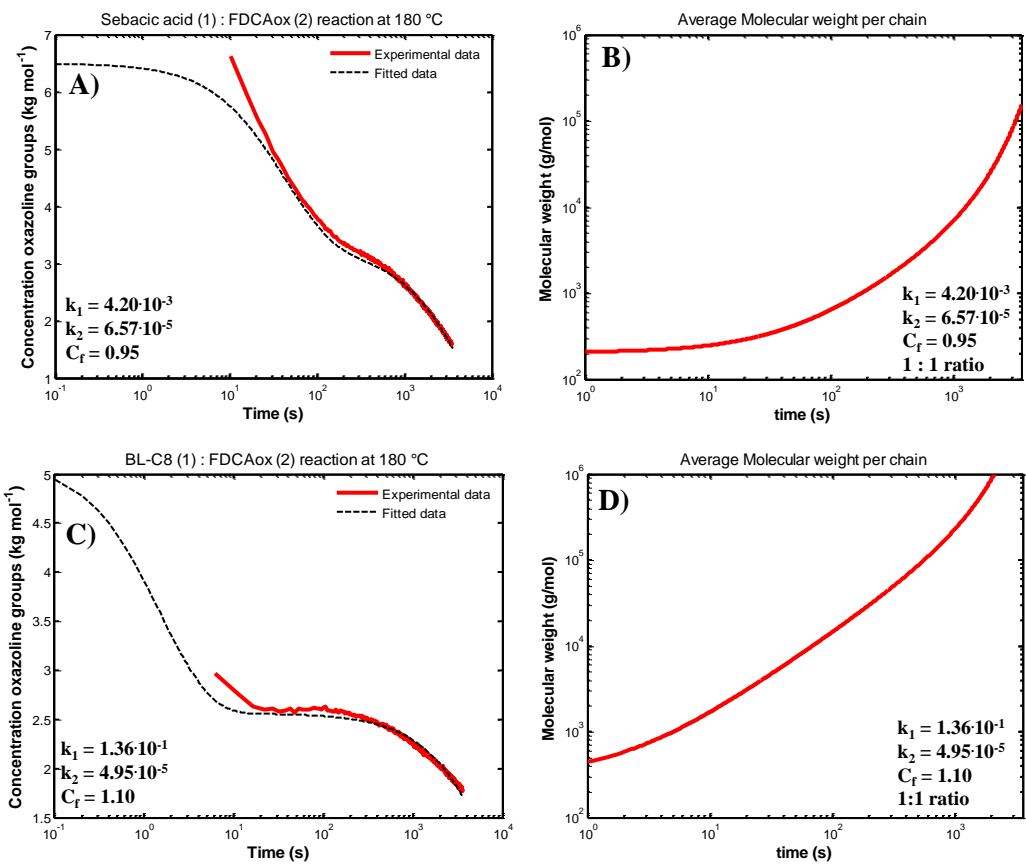

Figure A7. 2-Oxazoline depletion according to the FTIR data and corresponding data fit by using equations (1) - (3) ( $A$ and $C$ ) and the corresponding evolution of the average molecular weight during curing ( $B$ and $D$ ). Images $A$ and $B$ relate to the systems containing sebacic acid, whereas images $C$ and $D$ relate to the systems containing $B P-C_{8}$. 


\section{Reaction kinetics of FDCAox based systems containing various dicarboxylic acids}

As reported in the original manuscript, the curing of systems containing FDCAox (2) and various dicarboxylic acids (1) such as adipic acid or suberic acid (Figure A8) and $\mathrm{BP}_{-} \mathrm{C}_{4}$ or $\mathrm{BP}_{-} \mathrm{C}_{6}$ (Figure A10) was monitored on-line in FTIR at $180{ }^{\circ} \mathrm{C}$. Data fitting using the same procedure reported in the original manuscript confirms that both the reaction constants $k_{1}$ and $k_{2}$ for systems containing adipic acid or suberic acid are highly comparable with the reaction constants found for systems containing sebacic acid (Figure A9). Similarly, for systems containing BP$\mathrm{C}_{\mathrm{x}}$ monomers, the $\mathrm{k}_{2}$ reaction constant is comparable to the other dicarboxylic acids (Figure A11). As expected, the $k_{1}$ constants in systems containing $\mathrm{BP}-\mathrm{C}_{\mathrm{x}}$ are significantly higher than those found for systems containing adipic acid, suberic acid or sebacic acid. It is worthy to note that the $k_{1}$ constant for the system containing $\mathrm{BP}-\mathrm{C}_{4}$ is lower than for the $\mathrm{BP}-\mathrm{C}_{6}$ and $\mathrm{BP}-\mathrm{C}_{8}$ systems, which is a result from the system not being fully molten after 12 seconds, thus affecting the FTIR traces (Figure A10-A). This is in line with the observations reported in the manuscript, that most $\mathrm{BP}-\mathrm{C}_{\mathrm{x}}$ monomers are depleted already during the melting of the resin. 

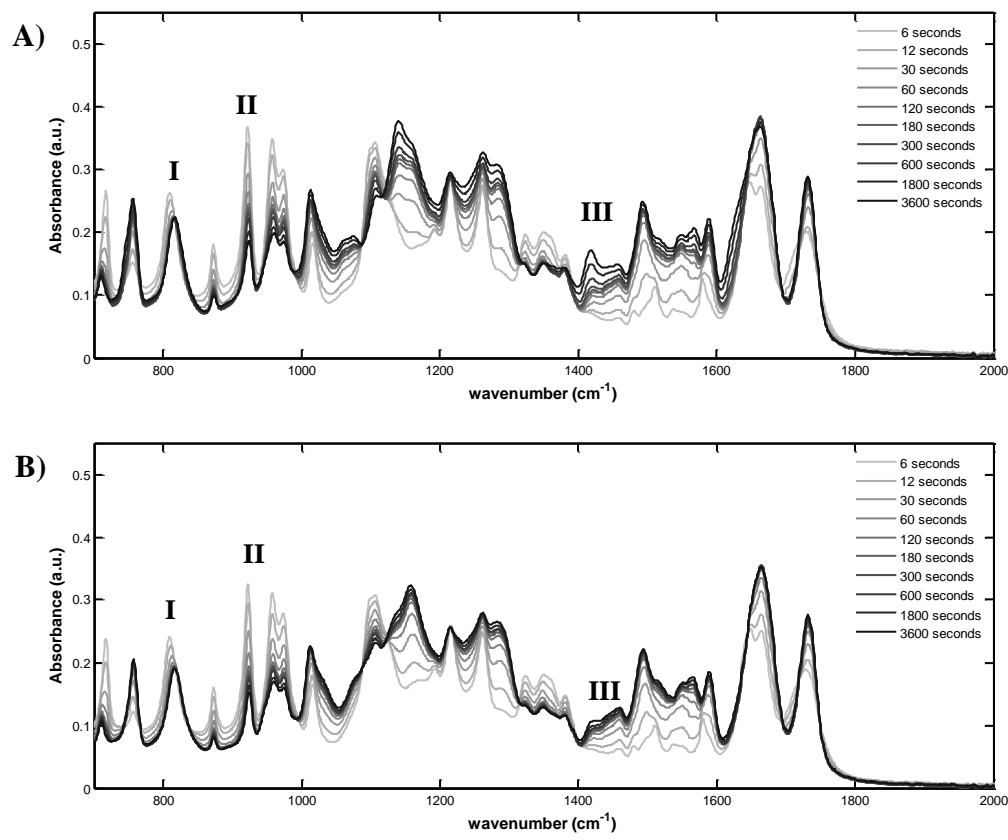

Figure A8. FTIR spectra (700-2000 $\left.\mathrm{cm}^{-1}\right)$ obtained during the on-line monitoring of the curing of (A) Adipic acid (1): FDCAox (2) and (B) Suberic acid (1) : FDCAox (2) systems. Vibrational bands I, II, and III denote the characteristic vibrations from the furan ring, 2-oxazoline ring, and tertiary amide bonds respectively.
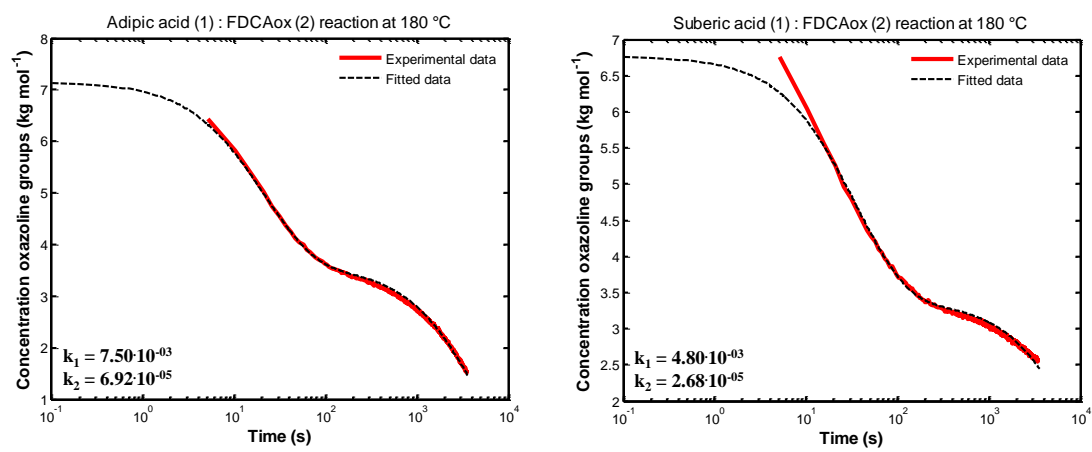

Figure A9. Experimental FTIR data and their fits of polymerizationf of left) FDCAox (2) : Adipic acid (1) and right) FDCAox (2) : Suberic acid (1) at $180{ }^{\circ} \mathrm{C}$. For both systems a conversion factor of 0.95 was used. 

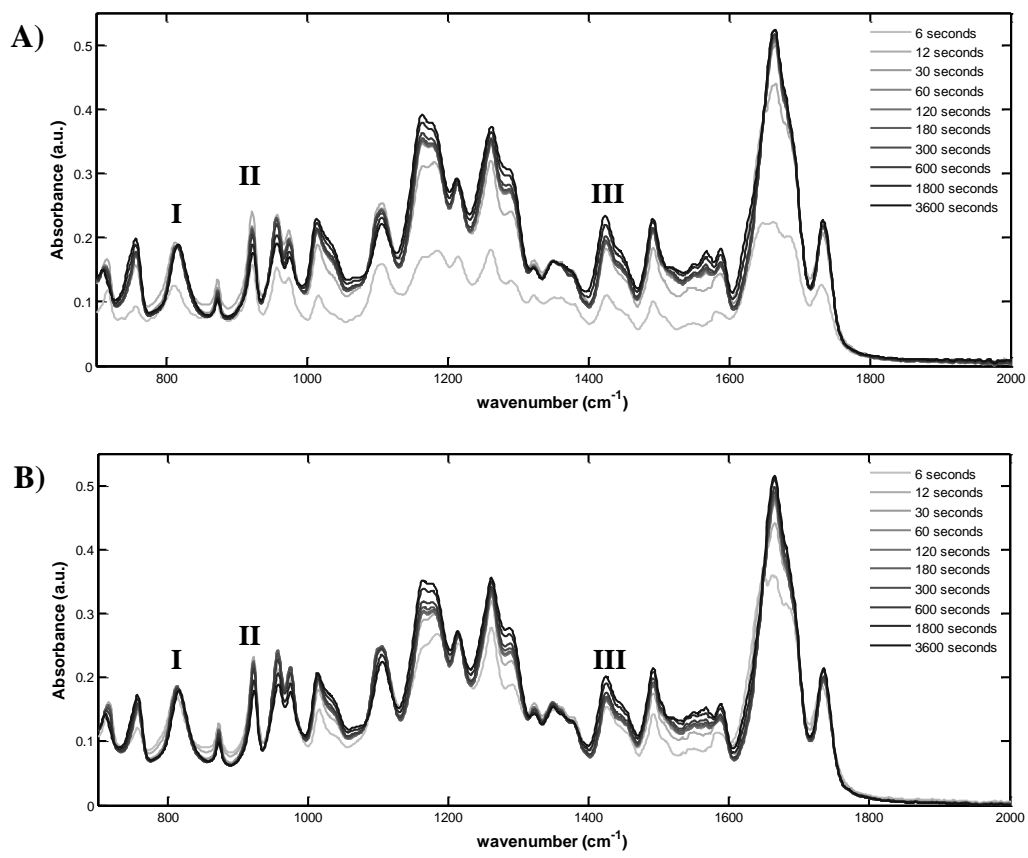

Figure A10. FTIR spectra (700-2000 $\left.\mathrm{cm}^{-1}\right)$ obtained during the on-line monitoring of the curing of (A) BP-C 4 (1): FDCAox (2) and (B) BP-C6 (1):FDCAox (2) systems. Vibrational bands I, II, and III denote the characteristic vibrations from the furan ring, 2-oxazoline ring, and tertiary amide bonds respectively.
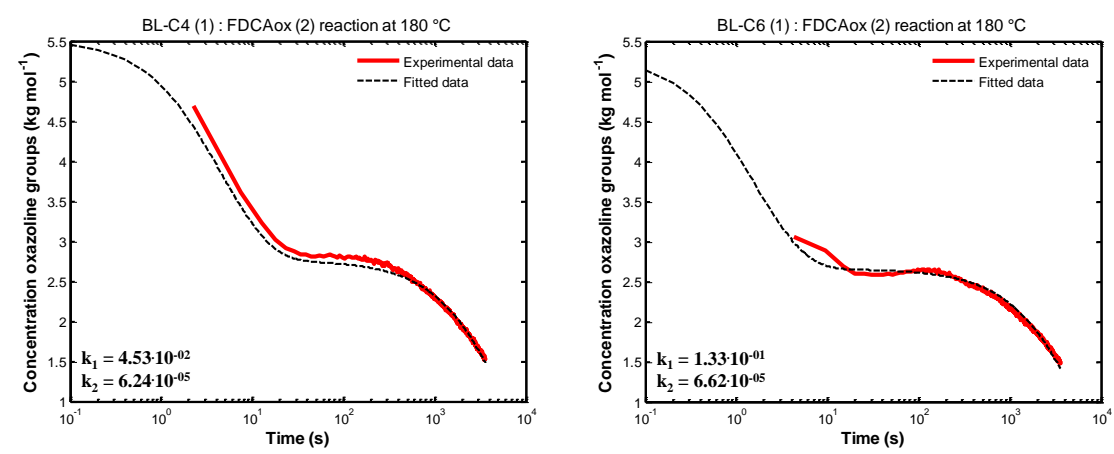

Figure A11 Experimental FTIR data and their fits of polymerizationf of left) FDCAox (2) : $\mathrm{BP}_{-} \mathrm{C}_{4}$ (1) and right) FDCAox (2) : BP-C6 (1) at $180{ }^{\circ} \mathrm{C}$. For both systems a conversion factor of 1.10 was used. 
2D NMR analysis of the synthesized (bis)pyrrolidone dicarboxylic acids are provided in the document below:

Scan the QR code to obtain the document.

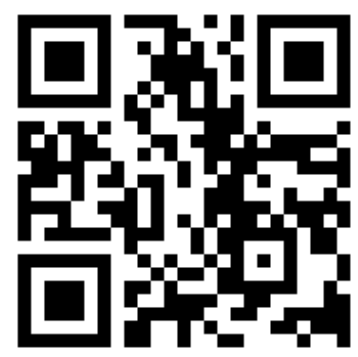




\section{APPENDIX B}





\section{Supporting information for Chapter 3}

\section{Analysis of $\mathrm{BP}-\mathrm{C}_{2}$ crystallites obtained after thermoset hydrolysis and consecutive crystallization.}

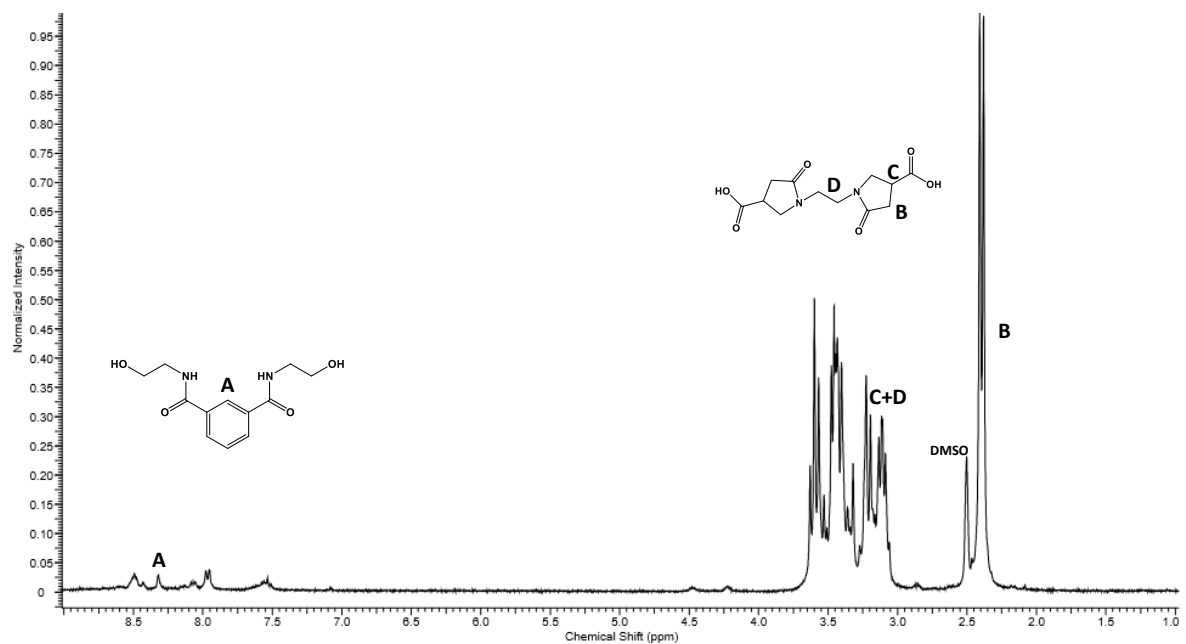

Figure B1. ${ }^{1} \mathrm{H}-\mathrm{NMR}$ spectrum of the BP-C $C_{2}$ crystallites obtained after thermoset hydrolysis and consecutive recrystallization. $B P-C_{2}$ purity was calculated by taking the ratio of signal A (1 proton from isophthalic acid) and signal $B$ (4 protons of the two pyrrolidone rings).

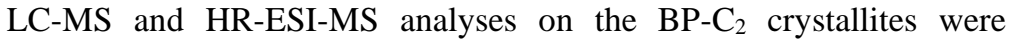
performed on an Agilent Technologies 1100 series LC/MSD system with a diode array detector (DAD) and a single quad MS. Analytical reversed phase HPLCanalyses were performed with a Phenomenex Luna C18 (2) column $(5 \mu \mathrm{m}, 250 \mathrm{~mm}$ $\times 4.6 \mathrm{~mm})$ and a solvent gradient $(0-100 \%$ acetonitrile with $0.1 \%$ Formic acid in $\mathrm{H}_{2} \mathrm{O}$ in $\left.15 \mathrm{~min}\right)$, the eluted compounds were analyzed via UV detection $(277 \mathrm{~nm})$. Mass spectrometry was used to confirm the molecular weight and elution time of the formed monomers. The results are depicted in Figures B2 and B3. 


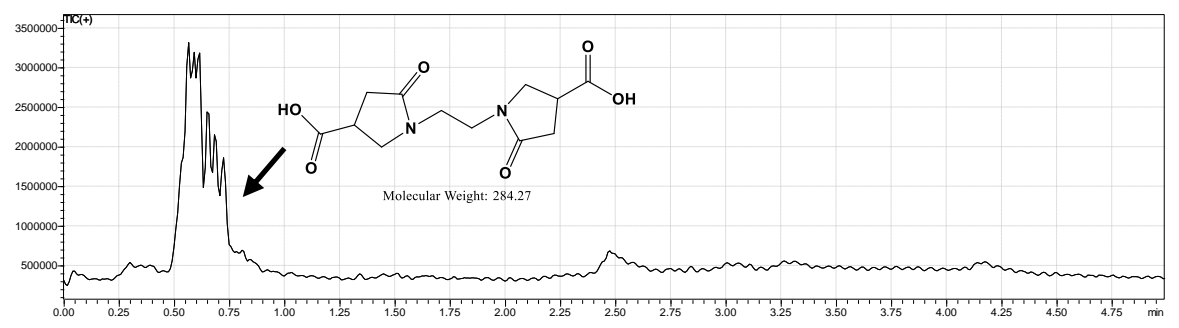

Figure B2. LC-MS chromatogram of the BP- $C_{2}$ crystallites obtained after thermoset hydrolysis and consecutive recrystallization.

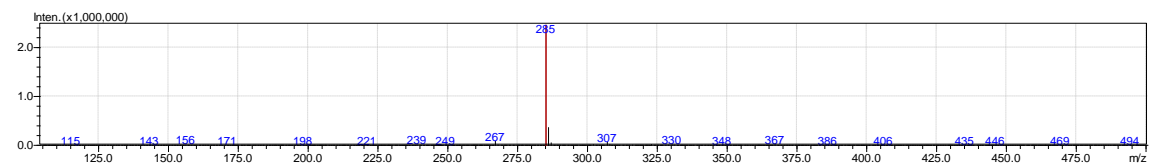

Figure B3. MS spectrum showing the molar mass of the compound eluting at 0.50.75 in the chromatogram shown in Figure S6. Note, the observed molecular weight $285(\mathrm{M+})$ corresponds to the molecular weight of a positively charged $\mathrm{BP}-\mathrm{C}_{2}$ molecule (with a $\mathrm{H+}$ ). 
APPENDIX C 



\section{Supporting information for Chapter 4}

\section{LC-MS traces of the reaction mixtures are shown below:}

\section{Synthesis of 1-(1-carboxy-2-phenylethyl)-5-oxopyrrolidine-3- carboxylic acid $(L-p h-a l a-P D)$}

The figure $\mathrm{C} 1$ depicts the elution of dimer $(\mathrm{m} / \mathrm{z}=294 \mathrm{~g} / \mathrm{mol})$ and itaconic acid within first 0.5 minute, followed with the elution of the desired $\boldsymbol{L}$-ph-ala-PD product $(\mathrm{m} / \mathrm{z}=278 \mathrm{~g} / \mathrm{mol})$ and intermediate $\mathrm{B}(\mathrm{m} / \mathrm{z}=278 \mathrm{~g} / \mathrm{mol})$ starting at 2.5 minutes. Additionally, the presence of decarboxylated $\boldsymbol{L}$-ph-ala-PD $(\mathrm{m} / \mathrm{z}=233$ $\mathrm{g} / \mathrm{mol}$ ) is also observed after 2.75 minutes. We consider it likely that the presence of the reaction intermediates and side-products are the cause of the relatively low isolated yield of $\boldsymbol{L}$-ph-ala-PD after purification.

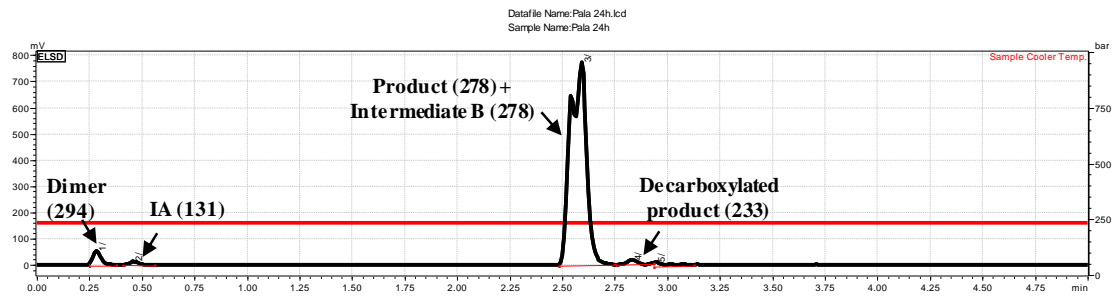

Figure $C 1$. LC-trace of crude reaction mixture of the reaction between $L$ phenylalanine and itaconic acid.

\section{Synthesis of 1-(1-carboxyethyl)-5-oxopyrrolidine-3-carboxylic acid $(L$-ala-PD)}

The figure C2 depicts the elution of the pyrrolidone $\boldsymbol{L}$-ala-PD based dimer (L-alanine) $(\mathrm{m} / \mathrm{z}=278 \mathrm{~g} / \mathrm{mol})$ already starting at 0.25 minutes. Furthermore, the product $(\mathrm{m} / \mathrm{z}=202 \mathrm{~g} / \mathrm{mol})$ elutes with the intermediate A $(\mathrm{m} / \mathrm{z}=224 \mathrm{~g} / \mathrm{mol})$. Despite the isolation of the $L$-ala-PD in the pure form according to NMR analysis, we consider it likely that the presence of both the pyrrolidone based dimer and the intermediate $\mathrm{A}$ in crude reaction mixture partly explain the relatively low yield after purification. 


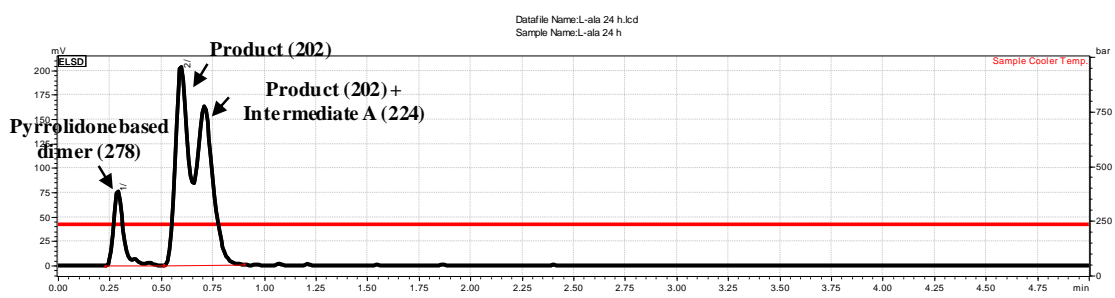

Figure C2. LC-trace of crude reaction mixture of the reaction between $L$-alanine and itaconic acid.

\section{Synthesis of 1-(2-carboxyethyl)-5-oxopyrrolidine-3-carboxylic acid ( $\beta$-ala-PD)}

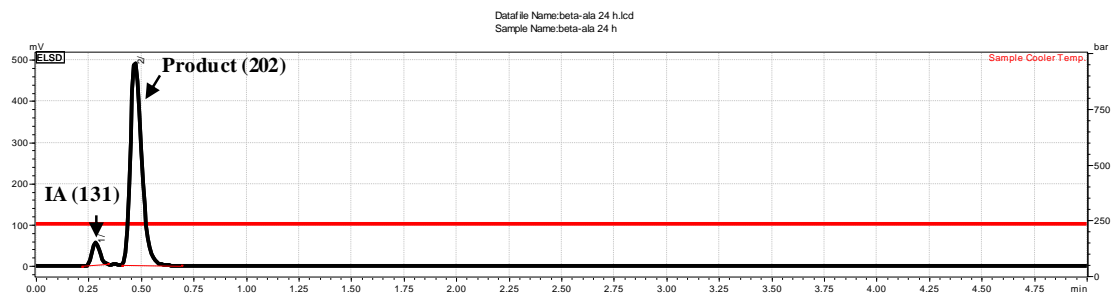

Figure C3. LC-trace of crude reaction mixture of the reaction between $\beta$-alanine and itaconic acid.

\section{Synthesis of 1-(3-carboxyethyl)-5-oxopyrrolidine-3-carboxylic acid ( $\gamma$-butyric-PD)}

From Figure $\mathrm{C} 4$ one can observe the presence of a small trace of itaconic acid, together with the pyrrolidone based dimer and imide as main side-products. Additionally, we observe the elution of a compound having $\mathrm{m} / \mathrm{z}=283 \mathrm{~g} / \mathrm{mol}$, which we expect to be the ring-closed pyrrolidone based dimer. Despite the high isolated yield of these monomers, it appears that there are some residual traces of the impurities present in addition to itaconic acid. 


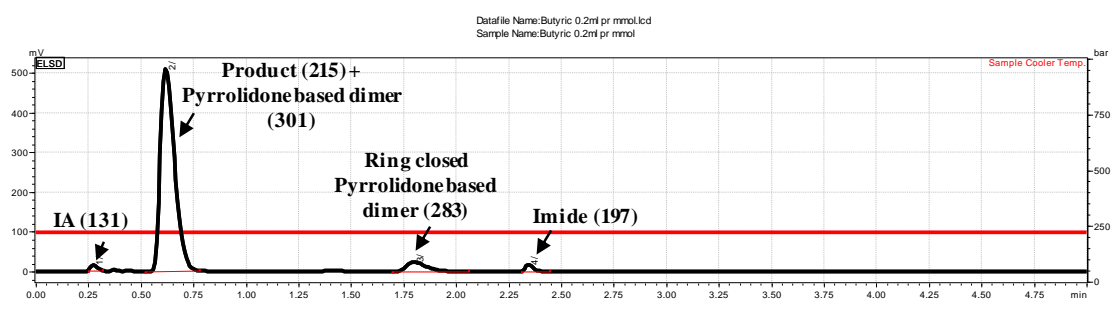

Figure C4. LC-trace of crude reaction mixture of the reaction between $\gamma$ aminobutyric acid and itaconic acid.

\section{Synthesis of 1-(10-carboxyethyl)-5-oxopyrrolidine-3-carboxylic acid (11-und-PD)}

From Figure C5, it is observed that the reaction between 11aminoundecanoic acid and itaconic acid results in the formation of the desired reaction product $(\mathrm{m} / \mathrm{z}=313 \mathrm{~g} / \mathrm{mol})$ which eluted at $2.75-3.0$ minutes. However, some trace of intermediate $A(\mathrm{~m} / \mathrm{z}=332 \mathrm{~g} / \mathrm{mol})$ is observed eluting at 3.1 minutes. Besides these, traces of imide $(296 \mathrm{~g} / \mathrm{mol})$ and intermediate $\mathrm{B}(\mathrm{m} / \mathrm{z}=314 \mathrm{~g} / \mathrm{mol})$ are observed in addition to a trace of imide side product $(\mathrm{m} / \mathrm{z}=296 \mathrm{~g} / \mathrm{mol})$.

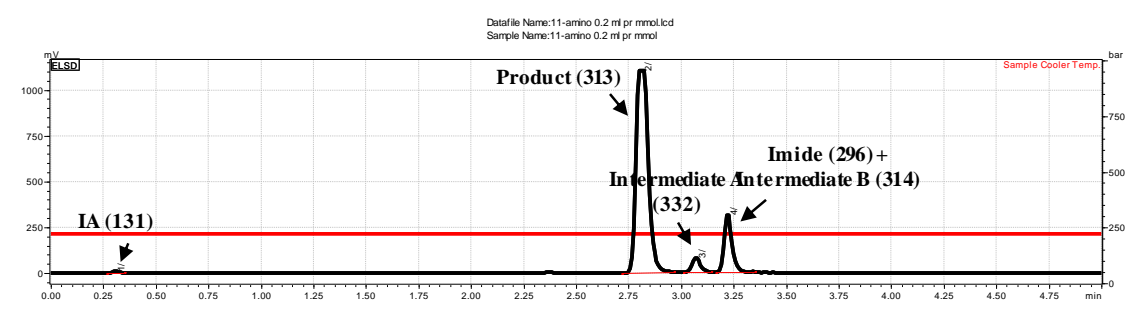

Figure C5. LC-trace of crude reaction mixture of the reaction between 11 aminoundecanoic acid and itaconic acid.

\section{Synthesis of 1-1'-(1-carboxypentane-1,5-diyl)bis(5- oxopyrrolidine-3-carboxylic acid ( $L$-lys-PD).}

As is shown in the LC-MS trace of the reaction product of the $\boldsymbol{L}$-lys-PD monomer, we observe the presence of two products; a disubstituted (major) and mono-substituted (minor) component. In case of the reaction mixture containing $L$ lysine (using a double molar ratio of itaconic acid, Figure C6), we observed that most functionalities participated in the aza-Michael addition-cyclization cascade reaction with itaconic acid, resulting in a minor amount of mono-, though mostly the disubstituted product $(\mathrm{m} / \mathrm{z}=371 \mathrm{~g} / \mathrm{mol}$.). Additionally, the presence of products where one of the amines reacted with the carboxylic acid of itaconic acid are anticipated 
$(\mathrm{m} / \mathrm{z}=371 \mathrm{~g} / \mathrm{mol}$, "Intermediate B"), as is supported by the elution of a compound with a mass of $\mathrm{m} / \mathrm{z}=353 \mathrm{~g} / \mathrm{mol}$ corresponding the imide side-product. The combination of various compounds in the reaction mixture make purification challenging. In addition, we noted that the L-lysine based products show excellent solubility in water, likely resulting contributing to higher losses during trituration.

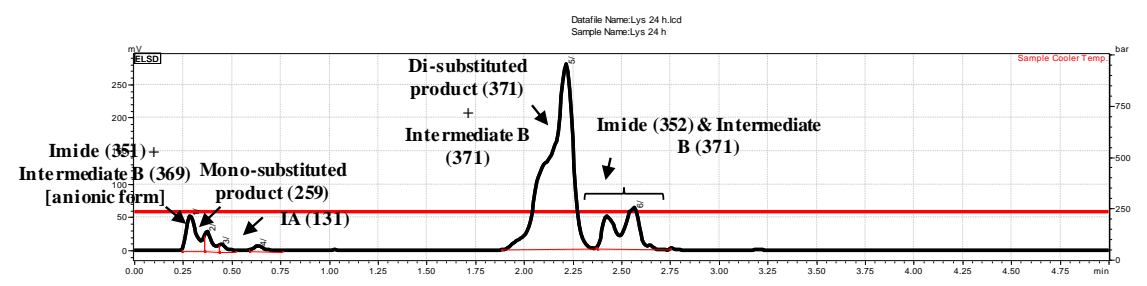

Figure C6. $L C$-trace of crude reaction mixture of the reaction between L-lysine and a double molar ratio of itaconic acid.

\section{Synthesis of 1-(1-carboxy-2-(3,4-dihydroxyphenyl)ethyl)-5- oxopyrrolidine-3-carboxylic acid (L-DOPA-PD)}

Additionally, we observe the elution of peaks having masses of $\mathrm{m} / \mathrm{z}=310$ g/mol ( $\boldsymbol{L}$-DOPA-PD or Intermediate B), $\mathrm{m} / \mathrm{z}=264 \mathrm{~g} / \mathrm{mol}$ (decarboxylated $\boldsymbol{L}$-DOPAPD or intermediate B) and $\mathrm{m} / \mathrm{z}=248 \mathrm{~g} / \mathrm{mol}$ (reduced decarboxylated form of product of $\boldsymbol{L}$-DOPA-PD) in figure C7. As observed from NMR analysis, the $L$-DOPA has a poor reactivity as residual $L$-DOPA remains present at the end of the reaction. Combined, the presence of various structures and the poor reactivity explain the challenging purification. As a result, we were unable to obtain the desired product in the pure form and excluded $\boldsymbol{L}$-DOPA-PD from further study.

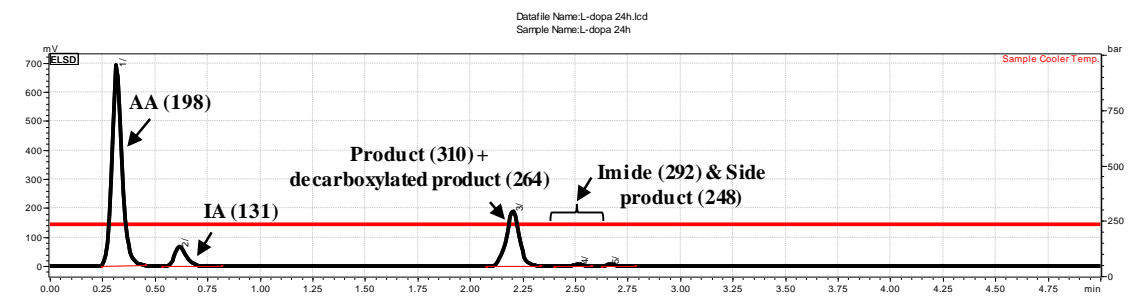

Figure C7. LC-trace of crude reaction mixture of the reaction between L-DOPA and itaconic acid. 


\section{Synthesis of 1-(1-carboxy-2-(1H-imidazol-5-yl) ethyl)-5- oxopyrrolidine-3-carboxylic acid ( $L$-his-PD)}

Similarly, the chromatogram of the reaction mixture of L-histidine and (a double molar ratio) itaconic acid, highlights the ability of the imidazole moiety to participate in the aza-Michael addition reaction (Figure C8). The presence of both the $\boldsymbol{L}$-his-PD product and its disubstituted alternative which did undergo an additional aza-Michael addition $(\mathrm{m} / \mathrm{z}=268$ and $398 \mathrm{~g} / \mathrm{mol}$, respectively), in line with findings from NMR analysis.
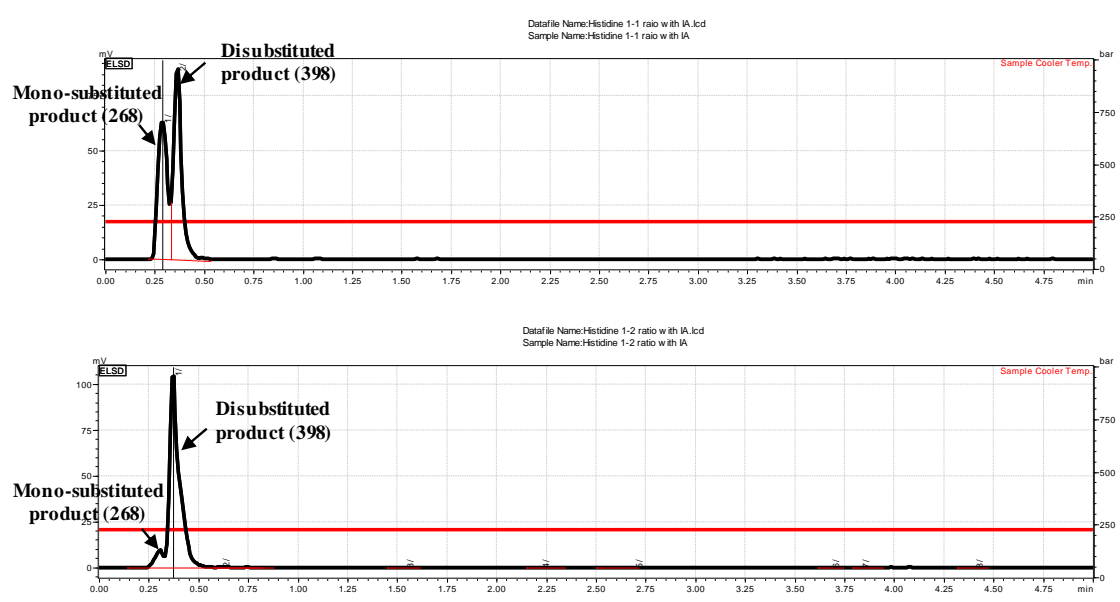

Figure C8. LC-trace of crude reaction mixture of the reaction between L-histidine and a double molar ratio of itaconic acid (top) and equimolar ratio of itaconic acid (bottom).

\section{2-oxazoline conversion determination procedure using NMR spectroscopy.}

To study the polymerization rate, the 2-oxazoline conversion was monitored during the polymerization of an equimolar ratio of 1,3-IAox with cotyrosinepolymer of $\left(\boldsymbol{L}\right.$-ph ala-PD $+\boldsymbol{L}$-tyr-PD), at $180^{\circ} \mathrm{C}$. Samples were collected after curing at 180 ${ }^{\circ} \mathrm{C}$ for an hour, dissolved in d-DMSO, and analyzed with ${ }^{1} \mathrm{H}$ NMR. To calculate the 2-oxazoline conversion, we divided the value of the integral of the $\mathrm{CH}_{2} \mathrm{O}$ resonance of the ring-opened 2-oxazoline ring by the total number of $\mathrm{CH}_{2} \mathrm{O}$ protons (of both reacted and unreacted 2-oxazolines, signals $\mathrm{X}$ and $\mathrm{Y}$, Figure $\mathrm{C} 9$ ). The total amount of 2-oxazoline was determined summing up the integrals of the $\mathrm{CH}_{2} \mathrm{O}$ resonance of the ring-opened 2-oxazoline ring (Signal Y, Figure C9) and the integral of the $\mathrm{CH}_{2} \mathrm{O}$ resonance of the non-reacted 2-oxazoline ring (Signal X, in Figure C9). 


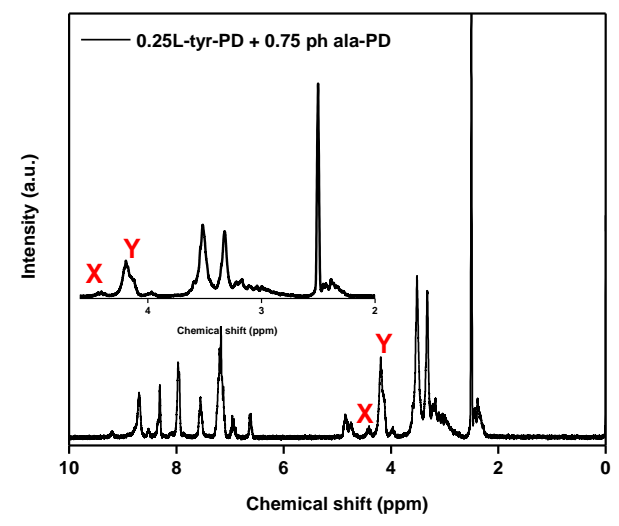

Figure C9. ${ }^{1} \mathrm{H} N \mathrm{~N} R$ spectrum in the range of $0-10 \mathrm{ppm}$ of the copolymerization product of IAox with L-tyr-PD and $\mathbf{L}$-ph ala-PD (1:3 molar ratio) after 20 minutes of curing (d-DMSO as solvent). $\mathrm{Y}$ and $\mathrm{X}$ highlight the $\mathrm{CH}_{2} \mathrm{O}$ resonance of the ringopened 2-oxazoline ring and the unreacted 2-oxazoline ring, respectively.

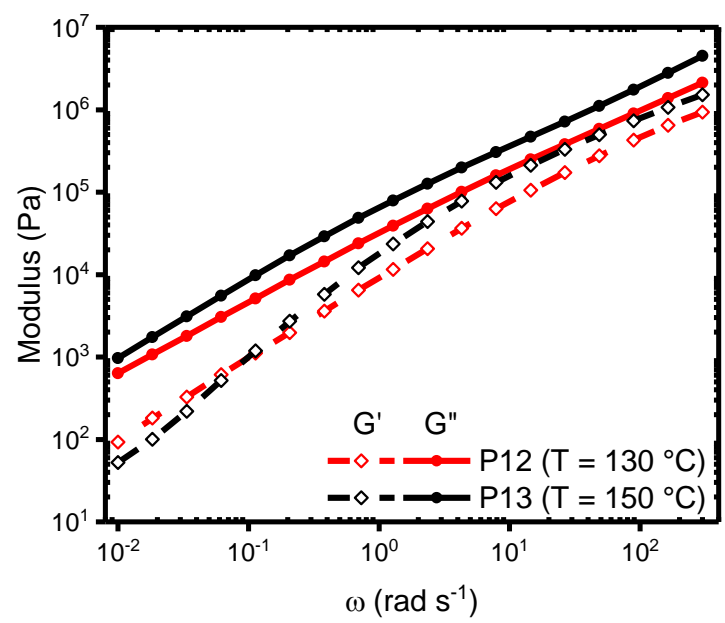

Figure C10. Frequency sweep of the polymer P12 $\left(130{ }^{\circ} \mathrm{C}\right)$ and P13 $\left(150{ }^{\circ} \mathrm{C}\right)$ determined at a strain of $0.1 \%$ and a frequency orange of 300-0.1 rad s-1. 


\section{TGA traces of synthesized monomers}

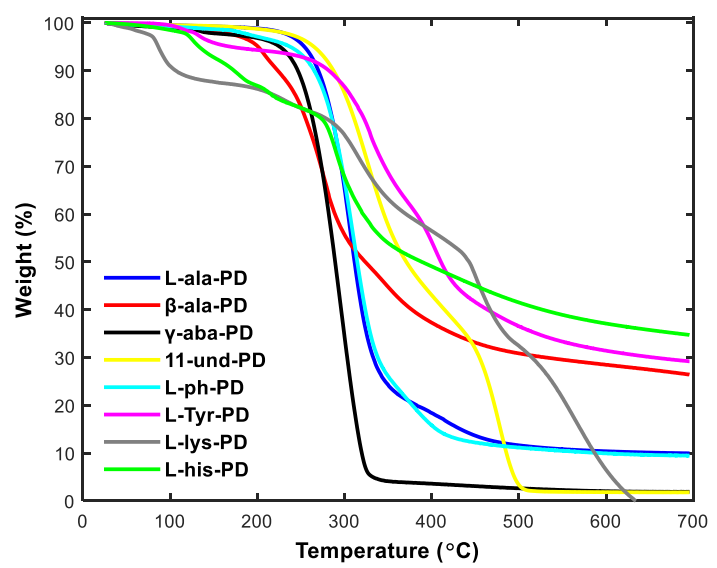

Figure C11. Weight loss as a function of temperature for the purified monomers developed in this work. TGA experiments were conducted under nitrogen atmosphere at a heating rate of $10{ }^{\circ} \mathrm{C} / \mathrm{min}$. Generally, we observe that all monomers are stable (ignoring the evaporation of some residual solvent) except for L-tyr-PD (showing 10 $\%$ wt loss around $150{ }^{\circ} \mathrm{C}$ owing to its slight thermal instability) with L-lys-PD and L-his-PD as exception. 


\section{DSC traces of synthesized polymers}
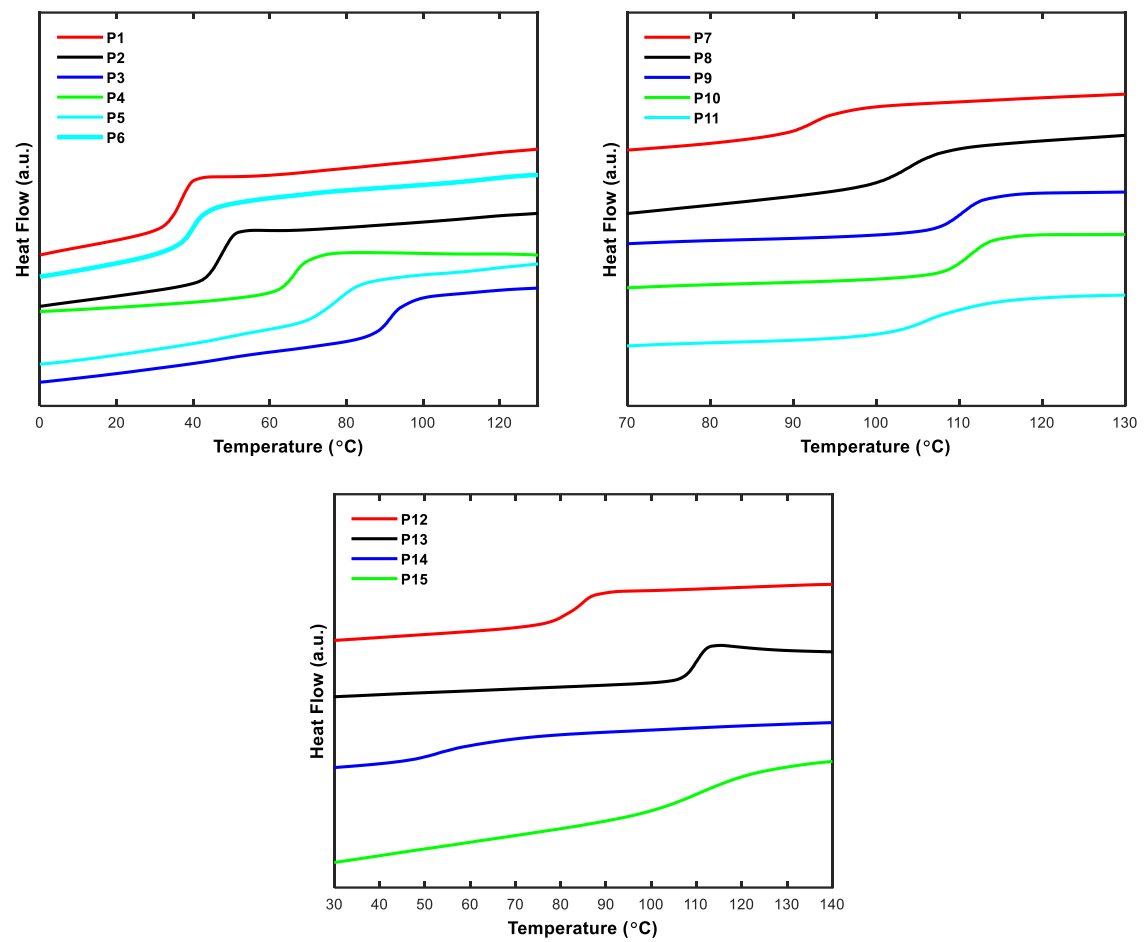

Figure C12. Second heating DSC traces of polymers P1 - P6 (left) and P7 - P11 (right) used for determination of the glass transition temperature, obtained during heating at a rate of $10^{\circ} \mathrm{C} / \mathrm{min}$. 
APPENDIX D 



\section{Supporting information for Chapter 5}

BHET morphology and its effect on PLA crystallization as a function of concentration

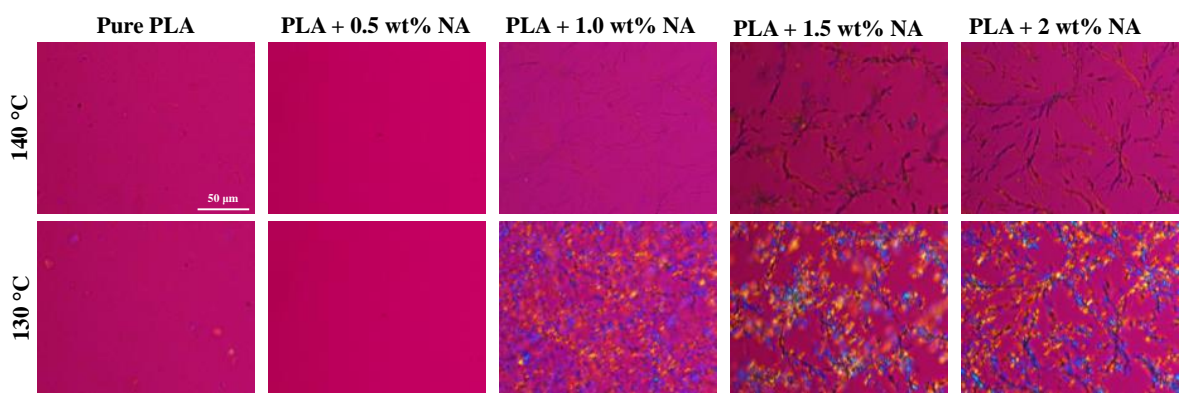

Figure D1. Optical micrographs taken between crossed polars and $530 \mathrm{~nm} \lambda$-wave plate of the samples having various concentration of BHET. The samples were first heated to $200{ }^{\circ} \mathrm{C}$ and cooled at a rate of $10^{\circ} \mathrm{C} / \mathrm{min}$. Note, for the sample having 2 $w t \%$ BHET, the sample was heated to $220{ }^{\circ} \mathrm{C}$ to enfore melting of BHET prior to cooling, despite potential degradation. 


\section{Isothermal crystallization after cooling at various rate (DSC)}
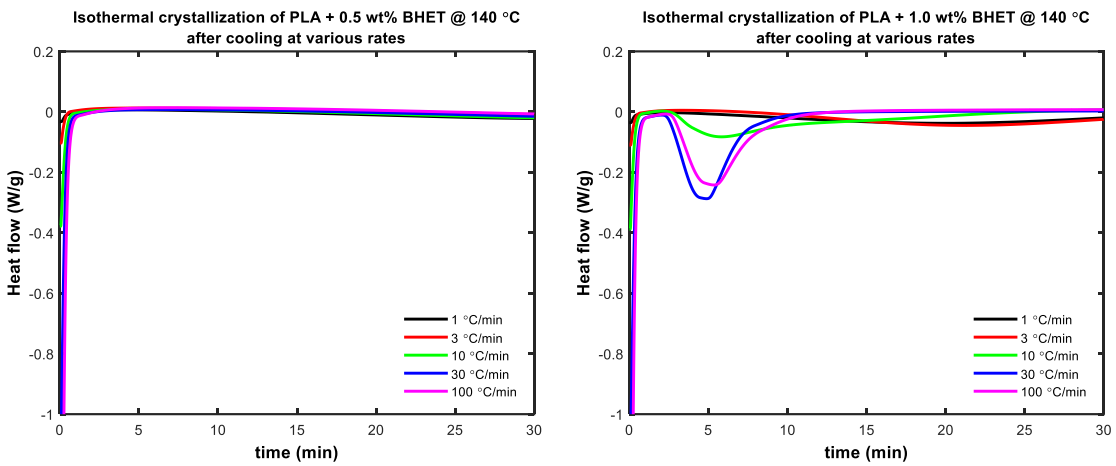

Figure D2. DSC thermograms observed during crystallization of PLA with 0.5 wt\% BHET (left) and $1.0 \mathrm{wt} \%$ BHET (right) under isothermal conditions at $140{ }^{\circ} \mathrm{C}$. Prior to crystallization, the samples were heated to $200{ }^{\circ} \mathrm{C}$ at a rate of $10^{\circ} \mathrm{C} / \mathrm{min}$, after which they were cooled with a specified cooling rate of $1,3,10,30$ and ' $100^{\circ}{ }^{\circ} \mathrm{C} / \mathrm{min}$ to $140{ }^{\circ} \mathrm{C}$.

\section{${ }^{1} \mathrm{H}-\mathrm{NMR}$ spectra of N,N'-bis(2-hydroxyethyl)terephthalamide (BHET)}

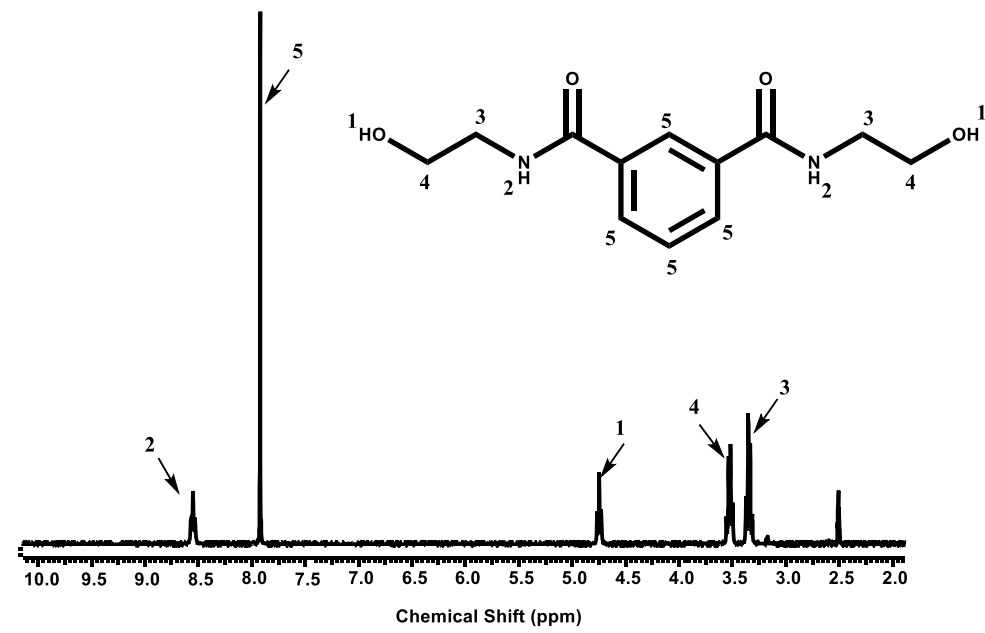

Figure D3. ${ }^{1} H$-NMR spectra of the polymer obtained by reacting $B P D C_{10}$ with FDCAox, cured at $180^{\circ} \mathrm{C}$ for 60 minutes, cured at $180^{\circ} \mathrm{C}$ for 60 minutes. 
${ }^{1}$ H-NMR spectra of N,N'-bis(2-hydroxyethyl)furan-2,5-dicarboxamide (BHEF)

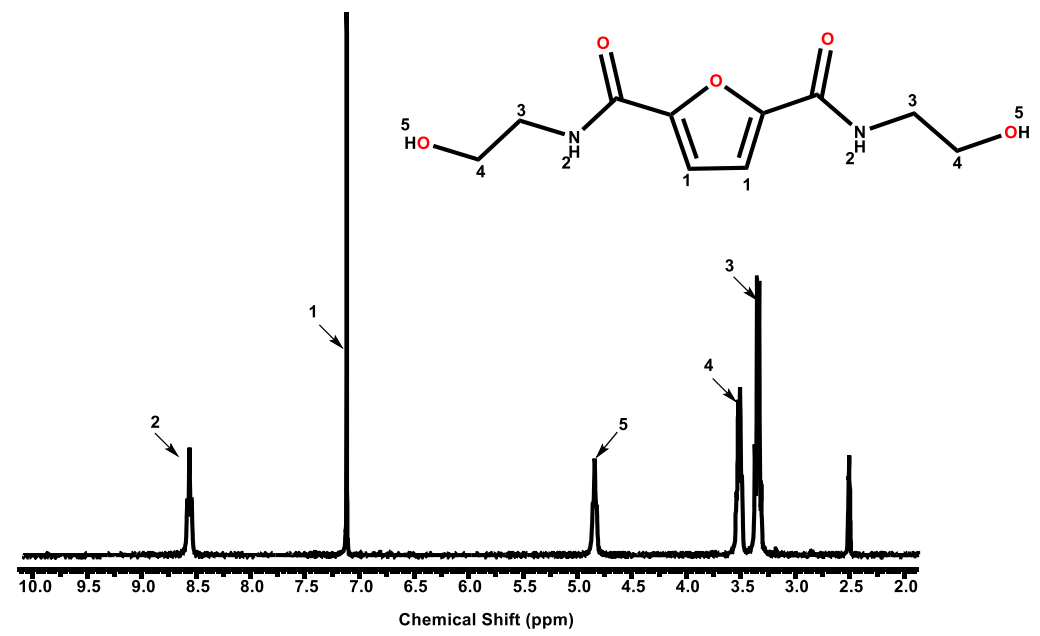

Figure D4. 'H-NMR spectra of the N,N'-bis(2-hydroxyethyl)furan-2,5dicarboxamide (BHEF) in deuterated DMSO as solvent.

Polarized optical microscopic images of the blend of PLA + BHEF (N,N'-bis(2-hydroxyethyl)furan-2,5-dicarboxamide)
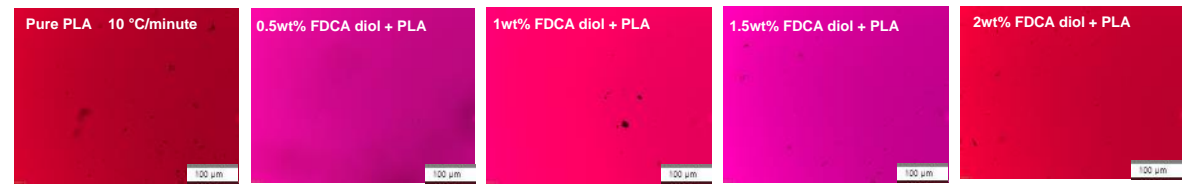

Figure D5. Polarized optical microscopic images (POM) of the bled of PLA + various wt $\%$ of FDCA diol extruded together at $190{ }^{\circ} \mathrm{C}$ for 3 minutes.

For microscopic images, a small amount of the samples are taken in between two microscopic cover slips and heated until $190{ }^{\circ} \mathrm{C}$ at a heating rate of $30{ }^{\circ} \mathrm{C} /$ minute, isothermal for 3 minutes and the cooled to room temperature at a cooling rate of 10 ${ }^{\circ} \mathrm{C} /$ minute. During cooling down to room temperature, there is no PLA crystal formation observed until $100{ }^{\circ} \mathrm{C}$. 



\section{APPENDIX E}





\section{Supporting information for Chapter 6}

\section{Identification of Rouse relaxation time for PLA}

The temperature dependence of the stretch and reorientation relaxation times of the PLA chains in the melt have been estimated using linear rheology experiments and tube modelling. In more detail, SAOS measurements have been performed at various temperatures on two PLA grades, an amorphous one (6302D) and a semicrystalline one (L130). For the amorphous grade temperatures range between $62.5^{\circ} \mathrm{C}$ and $200{ }^{\circ} \mathrm{C}$, probing chain dynamics from the glass transition to the melt. For the semi-crystalline grade temperatures range between $160{ }^{\circ} \mathrm{C}$ and $220{ }^{\circ} \mathrm{C}$ revealing the behavior of the chains in the melt. It is worth emphasizing that the two grades have similar molecular weight and molecular weight distributions, allowing us to predict the behavior of the L130 PLA grade based on the findings of the 6302D grade.

For both grades, Figure E1 presents master curves at four different temperatures in the melt. Black circles refer to the L130 grade while colored symbols refer to the amorphous grade. According to Figure E1, the high temperature responses of the semi-crystalline PLA and the amorphous PLA overlap. This finding strongly suggests that in both melts the polymer chains undergo the same reorientation dynamics. That is, at all temperatures from $160{ }^{\circ} \mathrm{C}$ to $220^{\circ} \mathrm{C}$, the crystalline structure of the L130 grade is molten and thus chain reorientation proceeds in the same way as in the amorphous grade. To a large extent, this is also the case at $140{ }^{\circ} \mathrm{C}$ since at this temperature the storage moduli of the blends, i.e. OBOCs with PLA, lie close to the modulus of pure PLA (see Figures 6.8 (right), 6.10 and 6.11). This fact justifies the use of the tube model for the estimation of the reptation and the Rouse relaxation times of the chains of the L130 grade, based on the findings for the 6302D grade. 

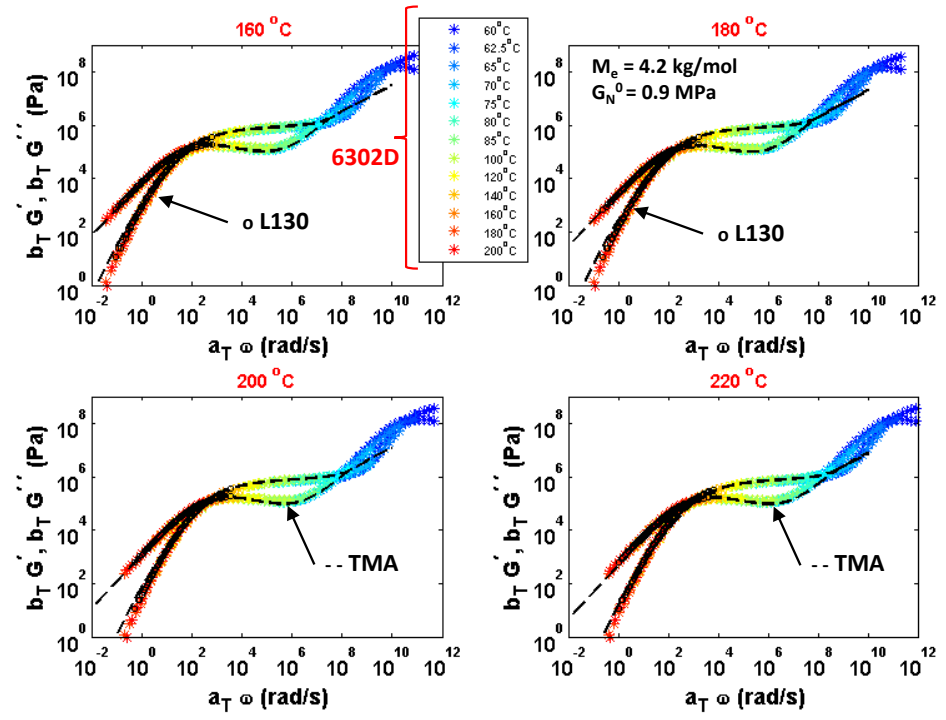

Figure E1. Master curves for an amorphous (6302D) and a semi-crystalline (L130) PLA grade at four temperatures in the melt. $\left(160^{\circ} \mathrm{C}, 180^{\circ} \mathrm{C}, 200^{\circ} \mathrm{C}\right.$, and $220^{\circ} \mathrm{C}$, respectively). Colored symbols refer to the $6302 \mathrm{D}$ grade while open black circles refer to the L130 grade. Dashed black lines represent tube model predictions using the experimental MWD of the 6302D material.

Notice that the master curves of both PLA grades have been obtained using identical shift factors. In particular, the horizontal and vertical shift factors respectively obey the following equations:

$$
\log a_{T}=\frac{-c_{1}\left(T-T_{r e f}\right)}{c_{2}+T_{r e f}}, \quad b_{T}=\frac{\left(\rho_{0}-T c_{3} 10^{-3}\right)(T+273.15)}{\left(\rho_{0}-T_{r e f} c_{3} 10^{-3}\right)\left(T_{r e f}+273.15\right)}
$$

where $c_{1}=3.0202, \quad c_{2}=-21.19{ }^{\circ} \mathrm{C}, c_{3}=0.69, \quad T_{\text {ref }}=180{ }^{\circ} \mathrm{C}$, and $\rho_{0}=$ $1.0752 \mathrm{~g} / \mathrm{cm}^{3}$.

When the reference temperature changes, the $c_{1}$ parameter of the WLF equation changes according to the expression:

$$
c_{1}^{\prime}=c_{1}\left(T_{r e f}+c_{2}\right) /\left(T_{r e f}^{\prime}+c_{2}\right) .
$$


The dashed lines in Figure E1 present tube model predictions using the experimentally determined MWD of the amorphous grade. $\overline{M_{w}}=210 \mathrm{~kg} / \mathrm{mol}$ and PDI $=1.7$. Model predictions at $180{ }^{\circ} \mathrm{C}$ have been obtained using the following parameterization set: $M_{e}=4.2 \mathrm{~kg} / \mathrm{mol}, G_{N}^{o}=0.9 \mathrm{MPa}$, and $\tau_{e}=5 * 10^{-8} \mathrm{~s}$. Model predictions at temperatures other than $180^{\circ} \mathrm{C}$ have been obtained by shifting the $\tau_{e}(T$ $=180^{\circ} \mathrm{C}$ ) value to the desired temperature using the WLF equation. Overall, the tube model describes well the experimental response of the 6302D grade. Since the L130 grade has very similar MWD, the same model predictions describe the behavior of the semi-crystalline PLA as well.

In this respect, the stretch and reorientation relaxation times of both PLA melts can be determined using the information provided by the tube model. More specific, the Rouse and reptation relaxation times of each chain of the distribution can be respectively estimated according to the expressions:

$$
\tau_{R_{i}}=\tau_{e} Z_{i}^{2}, \quad \tau_{r e p_{i}}=3 Z_{i} \tau_{R_{i}}\left[1-\left(3.38 / \sqrt{Z_{i}}\right)+\left(4.17 / Z_{i}\right)-\left(1.55 / Z_{i}^{3}\right)\right]
$$

with $Z_{i}$ denoting the number of entanglements, i.e. $Z_{i}=M_{w_{i}} / M_{e}$. In turn, an average Rouse and reptation time can be obtained utilizing the equations:

$$
\tau_{R}=\sum_{i=1}^{N} w_{i} \tau_{R_{i}}, \quad \tau_{\text {rep }}=\sum_{i=1}^{N} w_{i} \tau_{r e p_{i}}
$$

where $w_{i}$ is the (normalized) weight fraction of component (chain) $i$ of the molecular weight distribution.

Figures E2c and E2d show the temperature dependence of the obtained (average) relaxation times in the melt. In more detail, Figure E2c presents the Rouse or stretch relaxation times while Figure E2d depicts the reptation or reorientation relaxation times as a function of temperature. Open circles correspond to the average value when the whole MWD is accounted for. In the same figures, red and blue lines correspond to the situation at which only the highest $30 \%$ and $15 \%$ of the distribution is taken into account, respectively. These two fractions of the MWD can be seen in Figure E1a where the overall (normalized) MWD is shown by open circles. Finally, Figure E2b provides an alternative representation of the MWD; there the $y$ axis corresponds to $Z$, the number of entanglements, rather than weight fraction $w$. 

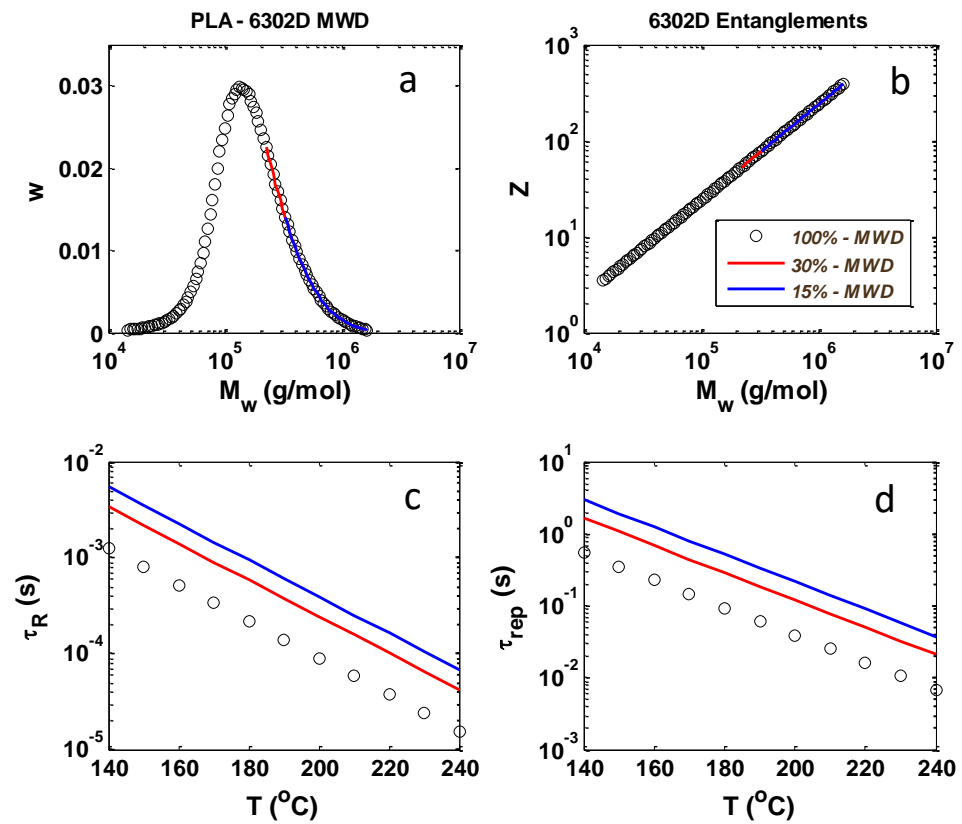

Figure E2. (a) Normalized MWD of the 6302D grade as obtained from GPC. (b) Molecular weights of the distribution as a function of the number of entanglements. (c) Temperature dependence of the average Rouse time of the distribution. (d) Temperature dependence of the average reptation time of the distribution. Open circles refer to the situation at which the whole MWD is considered in the calculations. Red and blue lines correspond to the situation at which the highest $30 \%$ and $15 \%$ of the MWD is only taken into account, respectively. 


\section{List of publications}

1) Roy, M.; Noordzij, G. J.; Van Den Boomen, Y.; Rastogi, S.; Wilsens, C. H. R. M. Renewable (Bis)Pyrrolidone Based Monomers as Components for Thermally Curable and Enzymatically Depolymerizable 2-Oxazoline Thermoset Resins. ACS Sustain. Chem. Eng. 2018, 6 (4), 5053-5066.

2) Roy, M.; Wilsens, C. H. R. M.; Leoné, N.; Rastogi, S. Use of Bis(Pyrrolidone)Based Dicarboxylic Acids in Poly(Ester-Amide)-Based Thermosets: Synthesis, Characterization, and Potential Route for Their Chemical Recycling. ACS Sustain. Chem. Eng. 2019, 7 (9), 8842-8852.

3) Roy, M; De Kort, G.W.; Reinartz, V.R.A.M; van Rijbroek, K; Van den Boom, A; Noordzij, G.; Würdemann, M. A.; Rastogi, S.; Wilsens, C.H.R.M. The Cascade AzaMichael addition-cyclization reaction; Development of pyrrolidone based dicarboxylic acids from naturally occurring amino acids, their polymerization, and its visco-elastic behavior (Manuscript submitted to Green Chemistry Journal).

4) Leoné, N.; Roy, M.; Saidi, S.; De Kort, G.W.; Hermida-Merino, D.; Wilsens, C. H. R. M. Improving Processing, Crystallization, and Performance of Poly-L-Lactide with an Amide-Based Organic Compound as Both Plasticizer and Nucleating Agent. ACS Omega, 2019, 4 (6), 10376-10387.

5) Roy, M; Zhelezniakov, M; De Kort, G.W.; Hawke, L; Leoné, N; Rastogi, S; Wilsens, C.H.R.M.; On the nucleation of polylactide by melt-soluble oxalamide based organic compounds. (Accepted and published in Polymer, 2020, https://doi.org/10.1016/j.polymer.2020.12268).

\section{Other publications by the author (not included in this thesis):}

1) Suckow, M.; Roy, M.; Sahre, K.; Häußler, L.; Singha, NK.; Voit, B.; Böhme, F.; Synthesis of polymeric ionic liquids with unidirectional chain topology by AB step growth polymerization, Polymer, 2017, 111, 123-129.

2) Suckow, M.; Mordvinkin, A.; Roy, M.; Singha, NK.; Heinrich, G.; Voit, B.; Tuning the Properties and Self-Healing Behavior of Ionically Modified Poly(isobutylene-co-isoprene) Rubber, Macromolecules, 2017, 51 (2), 468-479.

3) Kumar, ARSS.; Roy, M.; Singha, NK.; Effect of ionic liquids on the RAFT polymerization of butyl methacrylate, European Polymer Journal, 2018, 107, 294 302. 
4) Noordzij,GJ.; van den Boomen, YJG.; Gilbert, C.; van Elk, DJP.; Roy, M.; Wilsens, CHRM.; Rastogi, S.; The aza-Michael reaction: towards semi-crystalline polymers from renewable itaconic acid and diamines, Polymer Chemistry, 2019, 10 (29), 4049-4058.

5) Wilsens, CHRM.; Hawke, LGD.; De Kort, GW.; Saidi, S.; Roy, M.; Leoné, N.; Hermida-Merino, D.; Peters, W. M. G.; Rastogi, S.; Effect of Thermal History and Shear on the Viscoelastic Response of iPP Containing an Oxalamide-Based Organic Compound, Macromolecules, 2018, 52 (7), 2789-2802.

6) Noordzij, G.J.; Roy, M.; Bos, N.; Reinartz, V.; Wilsens, C.H.R.M.; Improving the Hydrolysis Rate of the Renewable Poly(hexamethylene sebacate) Through Copolymerization of a Bis(pyrrolidone)-Based Dicarboxylic Acid, Polymers, 2019, 11 (10), 1654-1668. 


\section{Acknowledgements "Dhanyavad"}

A journey of 4 years is coming to an end and I didn't realize how fast time flew. I still remember my first day of joining Aachen-Maastricht Institute of Biobased Materials (AMIBM) in 2016, which seems to be just yesterday. Through this journey of learning and personal development, I was supported by the people around me. It would be my immense pleasure to show my gratitude to my colleagues, friends and my supervisors, who have helped me during my stay. It has been a privilege to spend such a long time of my career and life with you all and make uncountable memories that I will cherish all through my life.

Firstly, I would like to acknowledge my supervisors, Dr. Karel Wilsens and Prof. Sanjay Rastogi. Thank you is a very small word to express my gratitude towards the guidance, shared knowledge and helping hand you both have showered on me during this journey. Sanjay, thank you for trusting me with the project and giving me the opportunity to perform my doctoral studies with you and your group in AMIBM. Your positive and encouraging words always helped me when I used to feel down. Really that's always what I have looked out for. Karel, thank you for everything, all your time, and our discussions. You have always been there from my day one till the end. I have learnt a lot and I can proudly count myself not only as a polymer chemist, but also as a materials expert, who knows what she is making.

Secondly, I would like to express my gratitude to my reading committee members Prof. Maarten Honing, Prof. Filip Du Prez, Prof. Katja Loos and Dr. Matt Baker, for their acceptance to become part of my committee and taking their valuable time to review my thesis. Thank you very much for your feedback.

Next comes our fabulous technical staff (Nils Leone, Jurrie Noordjik) with whom I had the pleasure to work for the last couple of years. There are many people who have played a vital role, directly or indirectly during my stay in Maastricht, especially Dr. Jules Harings, thank you for your advice in FTIR measurements, which was very helpful in my thesis work. Dr. Ketie Saralidze, I greatly appreciate your advice and helping hand while working with biobased fibers and, Yvonne van der Meer for your appreciation and kind words.

I would like to express my gratitude to all the members of my consortium, especially Stefan Hermanns for all the discussions and performing collaborative projects together, and the funding agencies for providing financial support. I am so grateful to be a part of this novel initiative taken towards finding a sustainable future.

Next, I would like to thank each of my student who I had the chance to supervise. You all have played a vital role in completion of my thesis. Vincent, Kim, Luca, Roxy, Daniel, Anouk, your hard work and perseverance have always admired me, which is visible in some parts of my thesis. Allegra, thank you for the last part of my thesis, where we have successfully showed the recycling efficiency of the 
regenerated bis(pyrrolidone) monomer. This can be a great scope of future research interest to pursue on. Megan, thank you for your hard work, in proving the concept of developing ionic bonding with mussel chemistry using pyrrolidone monomers, which is one of my favorite topics I have worked on during my research. I have enjoyed supervising you. I hope you all have gained and learnt as much as I did during your stay.

I would like to thank all my colleagues from AMIBM and Biobased Materials Groups for the great time we shared together, both inside and outside the campus, while enjoying coffee, drinks, party(ies) and all the tons of things we have done together. It has been one of my happiest part of my life. Thank you for all those laughs, deep conversations and all the fun we had together. I can now proudly say it loud that one glass of wine doesn't make me drunk anymore. Thank you, Milo, Ramiro, Ola, Marie, Martien, Naveen, Christian, Vahid, Pouya, Cris, Carlos and many others for the wonderful time, your support, help and suggestions. Laurence and Thomas, though we did not get too much time to work together, thank you for your suggestions and help. Laurence - Thank you for all the fun and help in learning rheology.

Now some special and close ones, Varun (chikku), Andrea (chotu), Gijs (goose), Geert (tyrex G) - thank you for all the everlasting memories, evening food and drinking nights. It has been a wonderful journey with you guys. Varun - I am still waiting when you will come to my lab and put acetone in any of my reactions...hahaha, thanks for forcing me out of lab for coffee. Bobo (amar chotu) I want to laugh with you again like the way you used to make me laugh in the stairs, can't wait to feel like that again, thanks. Gijs - my heartiest gratitude to you for being there for me, especially towards the end of my thesis. I could not have asked for a better teacher, you taught me rheology so well, you will always be my guru (teacher) in rheology. I loved working with you, hope we will be working together in future too. And, I am still waiting for you to scare me and get myself toppled off the chair....hahaha...those were such wonderful days. Geert- it took us sometime to know each other and become friends, but I am happy that it happened. Thanks for being there, starting as lab mates to lifelong friends. You will always be remembered whenever I will look back, thanks for your shoulder. I have two more person to add to the list - Monika and Anna. Thank you for those evenings and dinner, thank you for being there with me on my big day, in India. I hope you enjoyed a little among all the chaos. It meant a lot to me, my family and Anand. Few more people to add to this list, Nils, Lucienne, Rik, Christina, Yara - thank you all for all those fun evenings, movie nights and all those amazing things we have done together. Most importantly, I feel so blessed to have you guys in my life. Romy, thank you for being such a dear friend, I loved our movie nights, our outings, food and conversations, hope to see you soon in Sweden. 
Last but not the least, I wouldn't have been here without my parents. Thanks for believing in me, supporting me in pursuing my dreams, always encouraging me to follow my dream and what I want. Few words from you, bapi always gave me immense strength (Hard work always pays off at the end, if it is done with dedication, determination and discipline). Ma, thank you for always being there, and providing me with strength and mental support. I can't thank you enough. Golu (Kutty, my husband) - I won't say thank you, because I am nothing without you and you know that. So it is our life and we are defending actually for second time this same year...hahahah! I couldn't have come this far in life without your support and you beside me. Thank you for everything, let's now enjoy and explore our life together and see what life brings us. My parent in laws - Amma and appa - thank you for all your support and blessings. Maastricht has blessed me with another boon, Sarah (Franta - French Manta), whom I met just two years back and then gradually we bonded for life. We are truly two different persons but one soul. Thanks for coming into my life and becoming such a beautiful sister. You are the next best thing after Anand that has ever happened to me in my entire life. You gave me a family in France, where I feel like home. Ooppss....our bro is left out alone now! Ravi (bro) - thank you bro, although we couldn't get much time to spend together, but one year was enough for us to become friends. Bro I am still waiting for the spicy chicken curry!

I hope that I haven't missed out anyone, and if I have, kindly accept my apologies.

In this beautiful journey of life, hopefully we will again cross each other's path to make new memories... Stay happy and wish you all the best!

With lots of love and best wishes,

Manta 



\section{About the author}

Manta Roy was born in West Bengal, India in 1986. She did her bachelor studies in Chemistry from University of Calcutta, graduating in 2009. Manta obtained a Masters' degree in Organic Chemistry from the same University in 2011. That very year, she cleared Graduate Aptitude Test for Engineering to join for second Masters' in Polymer Science and Technology. However, later she decided to go for teaching and join as a lecturer of Organic Chemistry in Air Force Service Kendriya Vidyalaya and also part time lecturer in University of Calcutta (2011-

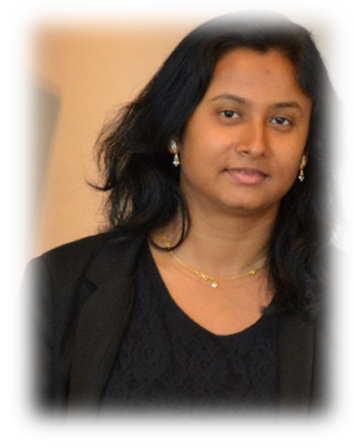
2013). She has several years of professional teaching experience, and thoroughly enjoyed the time being with young and fresh minds while training them. Later, she started developing more interest towards research, so she cleared an All India Entrance Test in 2013 to join Indian Institute of Technology in Kharagpur for a joint Masters' and Doctoral programme in the Department of Rubber Technology Centre and Polymer Science mostly focusing towards gaining expertise in polymer synthesis and characterization of thermoplastics, elastomeric blends and thermosets. She performed various research works under supervision of Prof. Nikhil K Singha while working on controlled free radical polymerization, ionic liquids and poly ionic liquids synthesis and polymer modifications to develop self-healing materials. The journey was an exhilarating experience, which is reflected from her academic performance that helped her to bag DAAD scholarship to perform her thesis in Leibniz Institute of Polymer Research (IPF) Dresden, Germany (2014) under supervision of Prof. Brigitte Voit and Dr. Frank Böhme. This opportunity exposed her to an international research environment, where she worked both as an organic and polymer chemist. She started developing expertise in materials science while working on the topic of dynamic networks. After successfully finishing the project, she went back to India to defend her thesis, where she received awards for best academic performance and her thesis in 2015. Later, she joined as a project researcher in Finland (2015-2016) in group of Prof. Mika Sillanpää, working on wastewater treatment. She resigned from her job in Finland in 2016 and joined Aachen-Maastricht Institute for Biobased Materials (AMIBM) to pursue her doctoral studies in Maastricht University, Netherlands under the supervision of Dr. Karel Wilsens and Prof. Sanjay Rastogi, which is now summarized in the form of a thesis to obtain her doctoral degree. During her stay, she has presented her research findings in several conferences and workshops and passed the RPK certified course on Polymer Physics: Module B organized by Dutch national postgraduate research school Polymeer Technologie Nederland. 
Starting her journey as a small-town girl has been turbulent, but perseverance has brought her to this platform today. During this course of time, she has developed a strong desire to pursue a career in contributing towards solving today's environmental problem. From the beginning of her research career, she has been inclined towards working on dynamic or reversible networks, which got further intensified after meeting and interacting with several great polymer scientists in a summer school on 'Dynamic and reversible polymer networks' in Bertinoro, Italy in May 2019, and while working for her thesis. Talking and listening to those great minds has made her more determined in pursuing her career towards developing recyclable and reprocessable polymer materials, which suits her research experience and skillset acquired over the years. From February 2020 onwards, she continues her research career in the field of reversible and dynamic networks working in Chalmers University of Technology as a researcher in the group of Prof. Christian Müller. 